\title{
Integration of NDE Reliability and Fracture Mechanics
}

Phase I Report

Prepared by F. L. Becker, S. R. Doctor, P. G. Heasler, C. J. Morris,

S. G. Pitman, G. P. Selby, F. A. Simonen

Pacific Northwest Laboratory

Operated by

Battelle Memorial Institute

Prepared for

U.S. Nuclear Regulatory

Commission 


\section{NOTICE}

This report was prepared as an account of work sponsored by an agency of the United States Government. Neither the United States Government nor any agency thereof, or any of their employees, makes any warranty, expressed or implied, or assumes any legal liability or responsibility for any third party's use, or the results of such use, of any information, apparatus product or process disclosed in this report, or represents that its use by such third party would not infringe privately owned rights.

Available from

GPO Sales Program

Division of Technical Information and Document Control

U. S. Nuclear Regulatory Commission

Washington, D. C. 20555

Printed copy price: $\$ 6.50$

and

Nâtional Technical Information Service

Springfield, Virginia 22161 


\section{Integration of NDE Reliability and Fracture Mechanics}

Phase I Report

Manuscript Completed: October 1980

Date Published: March 1981

Prepared by

F. L. Becker, S. R. Doctor, P. G. Heasler, C. J. Morris,

S. G. Pitman, G. P. Selby, F. A. Simonen

Pacific Northwest Laboratory

Richland, WA 99352

Prepared for

Division of Reactor Safety Research

Office of Nuclear Regulatory Research

U.S. Nuclear Regulatory Commission

Washington, D.C. 20555

NRC FIN B2289 


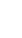




\section{ABSTRACT}

The Pacific Northwest Laboratory is conducting a four-phase prograin for measuring and evaluating the effectiveness and reliability of in-service inspection (ISI) perforined on the prinary system piping welds of commercial light water reactors ( $L W R s$ ). Phase I of the program is complete. A survey was inade of the state of practice for ultrasonic ISI of LWR primary system piping we ids. Fracture mechanics calculations were made to establish required nondestructive testing sensitivities. In general, it was found that fatigue flaws less than $25 \%$ of wall thickness would not grow to failure within an inspection interval of 10 years. However, in some cases failure could occur considerably faster. Statistical methods for predicting and measuring the effectiveness and reliability of ISI were developed and will be applied in the "Round Robin Inspections" of Phase II. Methods were also developed for the production of flaws typical of those found in service. Samples fabricated by these nethods will be used in Phase II to test inspection effectiveness and reliability. Measurements were made of the influence of flaw characteristics (i.e., roughness, tightness, and orientation) on inspection reliability. These measurements, as well as the predictions of a statistical model for inspection reliability, indicate that current reporting and recording sensitivities are inadequate. 



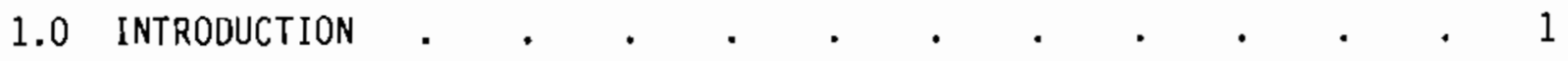

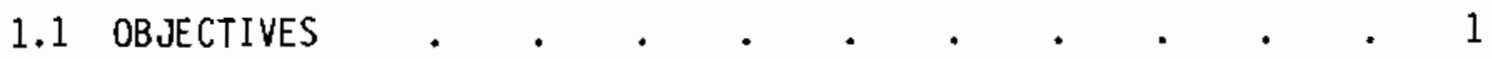

1.2 TECHNICAL APPROACH . . . . . . . . . . . . . 2

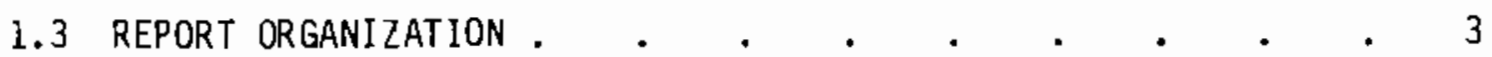

2.0 SUMMARY . . . . . . . . . . . . . . . . . . . 5

2.1 STATE-OF-PRACTICE REVIEW (SECTION 3) 3 ) $\quad . \quad+\quad . \quad$ • 5

2.2 FRACTURE MECHANICS ANALYSIS (SECTION 4) • • • • • • 6

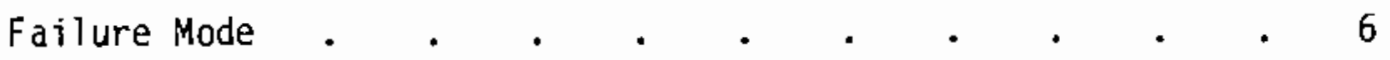

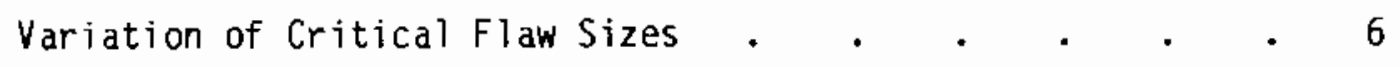

2.3 STATISTICAL OESIGN AND ANALYSIS (SECTION 5) . . . . 7

2.4 SAMPLE PREPARATION (SECTION 6) 6 .

2.5 MEASUREMENT AND EVALUATION (SECTION 7) 7 . $\quad . \quad . \quad . \quad . \quad 8$

3.0 STATE-OF-PRACTICE REVIEW OF ULTRASONIC IN-SERVICE
INSPECTION OF PRIMARY SYSTEM PIPING

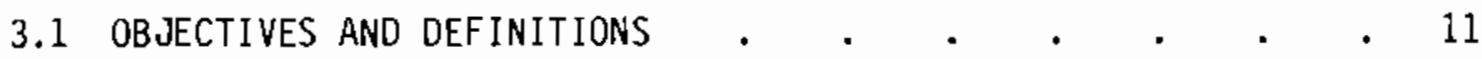

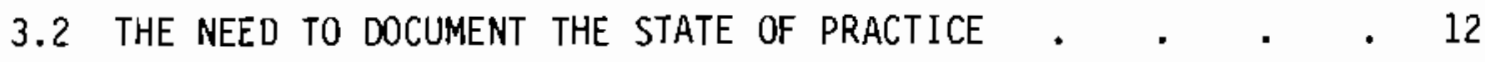

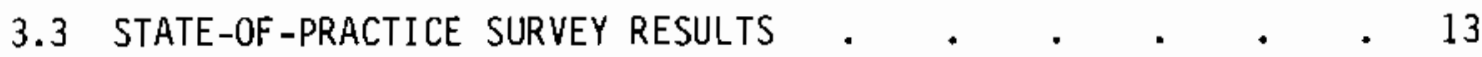

3.4 STATE-OF-PRACTICE SUMMARY AND CONCLUSIONS . . . . 27

4.0 FRACTURE MECHANICS ANALYSIS . . . . . . . . . . . . 31

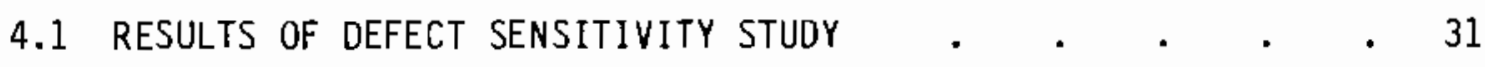

4.2 OVERVIEW OF CRITICAL FLAW SIZE ESTIMATES $\quad \cdot \quad \cdot \quad \cdot \quad \cdot \quad 32$

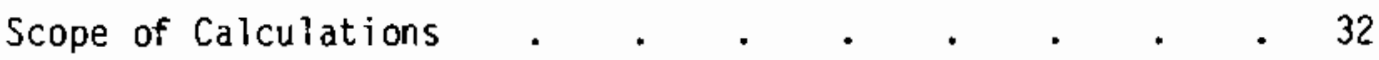

Conclusions of Flaw Size Estimates . . . . . . . 34

Failure Mode . . . . . . . . . . . 34 
Variation in Critical Flaw Sizes . . . . . . 35 ASME Code Evaluations . . . . . . . . 35 Tearing Instability Analysis . . . . . . . . 36

4.3 CRITICAL FLAWS BASEO DN FATIGUE . . . . . . 36

4.4 ASME SECTION XI FLAW SIZES . . . . . . . . 42

4.5 CRITICAL FLAWS BY DUAL CRITERIA APPROACH . . . • . 43 Axial Flaw - LEFM . . . . . . . . . 44

Axial Flaw - Net Section Collapse. . . . . . 45

Circumferential Flaws - LEFM . . . . . . . . . 47

Circumferentiai Flaws - Net Section Collapse . . . . . 48

4.5 TEARING INSTABILITY ANALYSES . . . . . . . . 49

Plastic Fracture Properties . . . . . . . 50

Deep Axial Surface Flaw . . . . . . . . . 51

Circumferential Flaw in Bending . . . . . . . 52

Circumferential Flaw in Tension . . . . . 54

5.0 STATISTiCAL DESIgN AND ANALYSiS . . . . . . . . . . . 61

5.1 MEASURES OF INSPECTION EFFECTIVENESS . . . . . . 51

Probability Estimates . . . . . . . . 61

False Call Rates . . . . . . . . 63

Measures of Association . . . . . . . . 64

5.2 ROUND ROBIN ULTRASONIC INSPECTION TEST . $\quad . \quad . \quad . \quad . \quad 67$

Pipe Materials and Flaws. . . . . . . . . . . 69

Round Rob in Test Conditions . . . . . . . . 70

Round Robin Statistical Design . . . . . . 73

Round Robin Experiment Structure and

Randomization . . . . . . . . . . 77 
Round Robin Analysis Procedures . . . . . . . 81

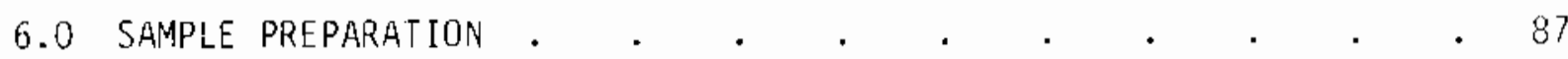

6.1 THERMAL FATIGUE CRACK GROWTH IN COMPLEX STRUCTURES . . 87

Stress Analys is of Thermal Fatigue Crack Growth . . . 89

Feasibility Study of Therma? Fatigue Crack

Growth in Stainless Steel . . . . . . . 89

Selection of Thermal catigue Procedures Uver

Mechanical Fatigue Procedures for Pipes . . . . . . 91

Preparation of Thermal Fatigue Cracking Specimens . . . 94

6.2 BENDING FATIGUE CRACK GROWTH IN FLAT PLATE SAMPLES • . 99

Crack Initiation for Bending Fatigue Samples . . . . . 102

Bending Fatigue Crack Growth . . . . . . . 103

Crack Length Measurement . . . . . . . . . 103

Control of Crack Aspect Ratius . . . . . . . 105

Crack Growth Conditions . . . . . . . . . 107

Results of Bending Fatigue Sample Preparation . . . . 107

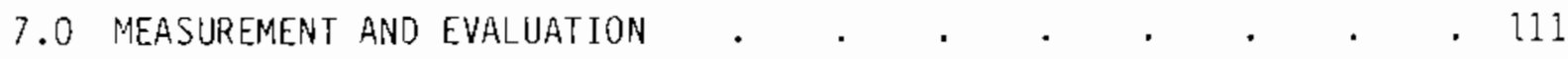

7.1 EFFECTS OF FLAW GEOMETRY ON INSPECTION RELIABILITY • • 11?

Notch Samples Prepared to Model Cracked Pipe . . . . 112

Transducers Used and Measurements Made . . . . . 113

Flaw Geometry Measurement Resuits . . . . . . . 114

Notch Detection in Simulated Thicker-Wall pipe . . . 128

Comparison of Code Calibration Reflectors . . . . 131

Summary: Impact of Flaw Geometry Results on
Inspection Reliability

\begin{tabular}{l}
7.2 EFFECTS OF FLAW CHARACTERISTICS ON INSPECTION \\
RELIABILITY \\
\hline
\end{tabular} 


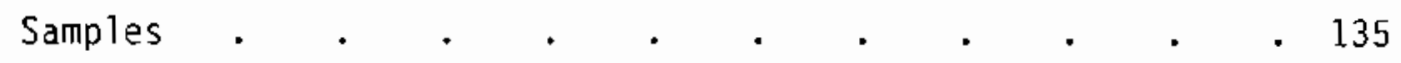

Flaw Measurement Results - Bending Fatigue

Samples . $. \quad . \quad . \quad . \quad . \quad . \quad . \quad . \quad . \quad 137$

Bending Fatigue Flaw Characteristics Effects

on Detectability . . . . . . . . . . . 140

Flaw Measurement Results - Thermal Fatigue Samples . . 155

Crack Depth Sizing . . . . . . . . . . 155

Thermal Fatigue Flaw Characteristics Effect on
Detectability. . . . . . . 157

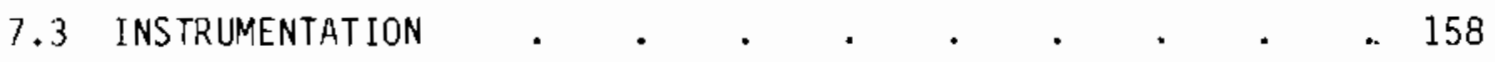

7.4 ESTIMATION OF FLAW OETECTION RELIABILITY $\quad . \quad$. $\quad$ • 160

Calculation of a Recoding Probability Curve from

dB-Response Information . . . . . . . . 163

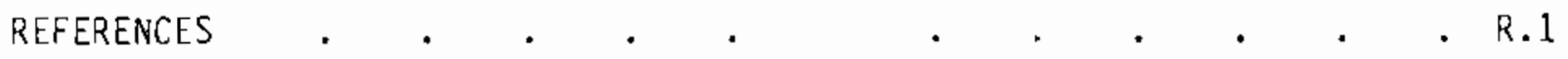

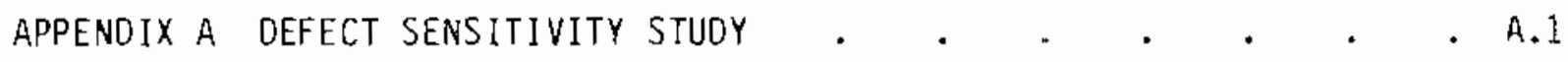

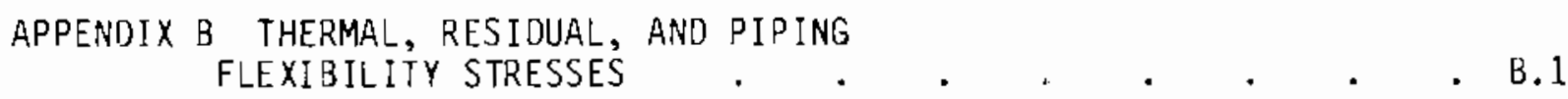

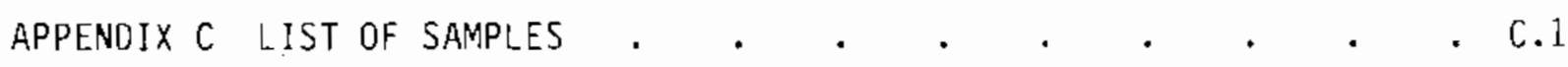

$\begin{array}{lll}\text { APPENOIX D } & \text { ANALYSIS OF THE THERMAL FATIGUE } \\ & \text { PROCESS FOR CARCKEO SPECIMEN PROOUCTION } & . \\ \end{array}$ 
3.1 Cross Section of a Typical welded Pipe Joint . . . . . . 13

3.2 Pipe Cross Section of Centrifugaliy Cast

Stainless Steel $. \quad . \quad . \quad . \quad . \quad . \quad . \quad . \quad . \quad 20$

3.3 Pipe Counterbore Geometry Conditions . . . . . . . . 24

4.1 Crack Growth in Cold Leg for 18-in. Long Axial Surface 38

4.2 Fatigue Crack Growth Data for Ferritic Steels . . . . . 40

5.1 Vector Representation of Weld Cross-Section . . . . . . 65

5.2 Construction of a Contingency Table $\quad . \quad$. . . . . . . . 67

5.3 Schedule of Experiments to be Performed Under Phase II

5.4 Data Matrix for Full ANOVA Experiment for One Team . . . $\quad$. 79

5.5 Data Matrix for Truncated ANOVA with Replication for

5.6 Data Matrix for Full ANOVA Without Replication

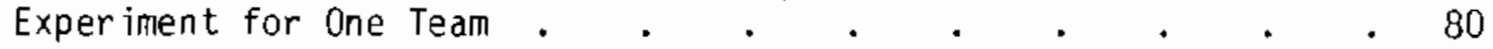

5.7 Data Matrix for Single Crack Size Experiment for

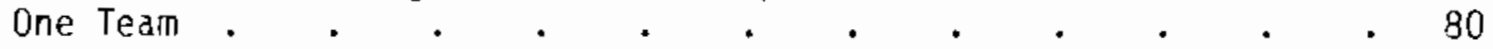

5.8 Required Number of Half Day Blocks for One Team . . . . . . $\quad$. 82

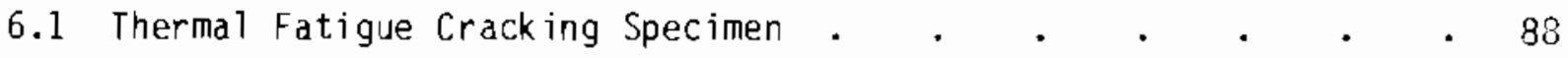

6.2 Thermal Fatigue Facility Block Diagram . . . . . . . . . . 90

6.3 Thermal Fatigue Crack in 304 S.S. . . . . . . . . . . 92

6.4 Mechanical Fatigue Facility for Multiple Welded 10-in.,
Schedule-80 Pipe (conceptual design) . .

6.5 Thermal Fatigue Facility . . . . . . . . . . . . 95

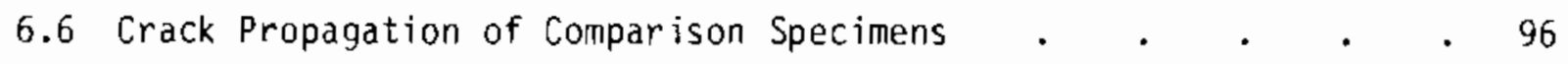

6.7 Crack Depth vs Thermal Cycles..$\quad$. . . . . . . . . . . 97 


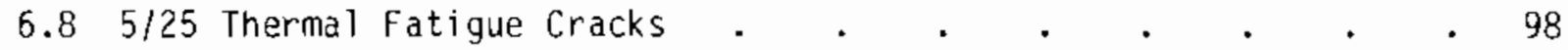

6.9 Aspect Ratio vs Crack Depth . . . . . . . . . 100

6.10 Thermal Fatigue Fixture for 10-in. Pipe . . . . . . 101

5.11 Specimen Fixture Used in Hydraulic Testing Machine . . . 102

6.12 Crack Surfaces Showing Low-Aspect-Ratio Initiator and Crack (Top) and High-Aspect-Ratio Initiator

and Crack (Bottom) . . . . . . . . . . . . . 104

6.13 Expanded View of Low-Aspect-Ratio Crack Face . . . . . . 106

7.1 Ultrasonic Response from Vertical Slits, $1 / 2 \vee$ Path, $45^{\circ}$ Beam . . . . . . . . . . . . . . . 116

7.2 Ultrasonic Response from Vertical Slits, Full $v$ Path, $45^{\circ}$ Beam . . . . . . . . . . . 117

7.3 U7trasonic Response from Vertical Slits, $3 / 2 \mathrm{~V}$ Path, $45^{\circ}$ Beam. . . . . . . . . . . 118

7.4 Ultrasonic Response from Vertical Slits, $1 / 2 \mathrm{~V}$ Path, $60^{\circ}$ Beam . . . . . . . . . . . . 119

7.5 Ultrasonic Response from Angled Slits, 100-mil Depth, $1 / 2$ V Path, $45^{\circ}$ Beam . . . . . . . . . . 120

7.6 Uitrasonic Response from Angled Si its, 100-mi] Depth, Full V Path, $45^{\circ}$ Beam . . . . . . . . . 121

7.7 Ultrasonic Response from Angled Slits, 100-mil Depth, $3 / 2$ V Path, $45^{\circ}$ Beam . . . . . . . . . . . 121

7.8 Ultrasonic Response from Angled Slits, 100-mil Depth, $1 / 2$ V Path, $60^{\circ}$ Beam . . . . . . . . 12?

7.9 Ultrasonic Response from Vertical, 100-mil Slits
in Angled Back Surfaces, $1 / 2$ V Path, $45^{\circ}$ Beam . . . . 123

7.10 Ultrasonic Response from Vertical, 100-mil Sli:s
in Angled Back Surfaces, $1 / 2$ V Path, $60^{\circ}$ Beam . . . . . 124

7. 11 Reflection Coefficient for Shear Wave Incident Upon Steel-to-Air Interface, After Arenburg . . . . . 125

7.1? Ultrasonic Response from Angled Slits, All Deptis,
$1 / 2$ V Path, 45 Beam $. \quad . \quad . \quad . \quad . \quad . \quad . \quad 125$ 
7.13 Ultrasonic Response from Angled Slits, All Depths, Full V Path, $45^{\circ}$ Beam . . . . . . . . . 127

7.14 U1trasonio Response from Angled Slits, All Depths, $3 / 2$ V Path, $45^{\circ}$ Beam . . . . . . . . . 128

7.15 Ultrasonic Response from Angled Slits, All Depths, $1 / 2$ V Path, $60^{\circ}$ Beam . . . . . . . . 129

7.15 Ultrasonic Response from Vertical Slits in Angled Back Surfaces, A1) Depths, $1 / 2$ V Path, $45^{\circ}$ Beam . . . . 130

7.17 Ultrasonic Response from Vertical Slits in Angled Back Surfaces, All Depths, $1 / 2$ V Path, $60^{\circ}$ Beam . . . . 131

7.18 Simulated Inspection of Thicker Material with $1 / 4$-in. Transducer . . . . . . . . 13 ?

7.19 Simulated Inspection of Thicker Material with 1/2-in. Transducer . . . . . . . . . . 133

7.20 Simulated Inspection of Thicker Material with ?-in. Transducer . . . . . . . . . . . 134

7.21 Satellite Pulse Technique for Crack Sizing . . . . . . 138

7.22 Electrical Resistance Gauge Performance in Measurement of Bending Fatique Crack Depth . . . . . . . . 139

$7.23-6$ dB Probe Motion in Shadow Technique Inspection of Bending Fatigue Cracks . $. .5 . \quad . \quad . \quad . \quad 140$

7.24 -20 dB Probe Motion in Pulse-Echo Inspection of Bending Fatigue Cracks . . . . . . . . . . . 141

7.2501 trasonic Response of Vertical EOM S1 its and Bending Fatigue Cracks, $1 / 2$ V Path, 45 Beam . . . . . 142

7.26 Theoratical Ultrasonic Reflection Coefficient from Plane Paraliel Stainless Steel Plates Separated by Air and water, for 2.25- MHz Shear wave at $45^{\circ}$

7.27 Four-Point Bending Fixture, Ultrasonic Inspection Instrument, and Transducer . . . . . . . . 145

7.28 Ultrasonic Response of Bending Fatigue Crack A153 with Varying Force Applied to Four-Point Bending Fixture 
7.29 U7trasonic Response of Bending Fatigue Crack A161 with Varying Force Applied to Four-Point Bending

7.30 Uitrasonic Response of Bending Fatigue Crack A141 with Varying Force Applied to Four-Point Bending

Fixture

7.31 Change in Ultrasonic Response of Bending Fatigue

Cracks Upon Compression to $75 \%$ of Yield Stress

7.32 Ultrasonic Response of Bending Fatigue Cracks

Compressed to $75 \%$ of Yield Stress.

U1trasonic Response of Bending Fatigue Crack A155

(Annealed) with Varying Force Applied to Four-Point

Bending Fixture

7.34 U1trasonic Response of Bending Fatigue Crack A156

(Annealed) with Varying Force Applied to Four-Point

Bending $F$ ixture

7.35 Uitrasonic Response of Thermal Fatigue Crack 8103 with Varying Force Applied to Four-Point Bending

Fixture

7.36 U?trasonic Response of Thermal Fatigue Crack B105 with Varying Force Applied to Four-Point Bending

Fixture

7.37 Ultrasonic Response of Thermal Fatigue Crack B113

(Annealed) with Varying Force Applied to Four-Point

Bending Fixture

7.38 Ultrasonic Response of Thermal Fatigue Crack B116

(Annealed) with Varying Force Applied to Four-Point

Bending Fixture

7.39 Response from 0.1 in. Deep Angled Back Surface Notches

Using the Same Search Unit and Two Different but

Similar UT Instruments

7.40 Adjusted Response Cuve for Fatigue Flaws Under Compression, Shown with $2 \sigma$ Error Bounds . . . . . . . 166

7.41 Estimated Recording Probability (i.e., the probability that the ultrasonic response will exceed a threshold value) Versus Flaw Depth for 100,50 , and $20 \% \mathrm{DAC}$

Recording or Reporting Thresholds 
7.42 Measured Recording Probability Versus Depth for 100, 50, and 20\% DAC Thresholds Reported by Forli (1979) 
4.1 Reactor Coolant Systern Transients . . . . . . . . . . . 38

4.2 Critical Flaw Size Estimates Based on

Fatigue Crack Growth . . . . . . . . . . 41

4.3 Critical Flaw Size Estimates Based on ASME Section $X I$ for Interna? Surface

Flaws

4.4 Critical Lengths of Through-wall Flaws in Coid Leg as Predicted by Dual Criteria Approach . . . . . . 46

4.5 Plastic Fracture Properties Used in Flaw Evaluations $\left(550^{\circ} \mathrm{F}\right)$. $. \quad . \quad . \quad . \quad . \quad . \quad . \quad . \quad . \quad 51$

4.6 Critical Pipe Lengths for Pipes With Circumferential Flaw Loaded by Displacement Controlled Bending Loads . . . 53

4.7 Critical Flaw Depths and Critical Pipe Lengths for Unstable Tearing of Deep 360-Degree Circumferential Flaw in Pipe Loaded in Axial Tension . $. \quad . \quad . \quad . \quad . \quad 58$

4.8 Critical Flaw Depths for 20 Foot Length Pipe by Ductile Tearing for Deep 360-Degree Circumferentia? Flaw Loaded in Axial Tension . . . . . . . . . . . 59

5.1 Conditions Under Investigation in the Phase IJ Round Robin Ultrasonic Inspection Test . . . . . . . 74

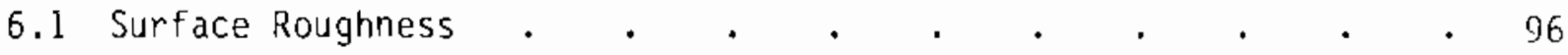

6.2 Specimen Matrix for Flaw Characteristics Experiment . . . 108

7.1 Decibe 1 Responses of a $10 \%$ Notch Compared to a Side-Drilled Hole DAC . . . . . . . . . . 132

7.2 Yield Stress of Cold-Rolled and Annealed Samples . . . . . 137

7.3 ERG Depth Measurement Performance on Therma1

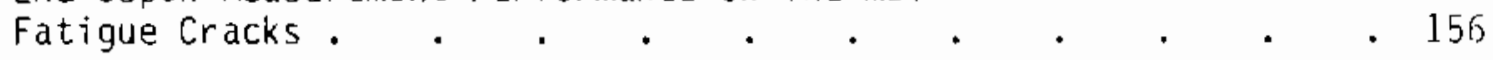

7.4 Estimate of Standard Deviation of Inspection Variables. 


\subsection{INTRODUCTION}

In-service inspection of commercial light water reactor primary piping systems is performed to meet the requirements of Section XI of the American Society of Mechanical Engineers (ASME) Boiler and Pressure Vessel Code (hereafter referred to as Code) and Appendix III of Section XI (ASME 1977). Periodic inspections of a sampling of pipe joints are made 1) to assure that cracks are not growing while pipes are in service and 2) to identify generic defects which may be present in the remainder of the system or in similar systems.

The adequacy of ASME Section $X I$ requirements for assuring continued operating safety has not been conclusively established. Safety analyses therefore give little credence to the ability of in-service inspection (ISI) to detect defects that may result in pipe failure. This and other programs sponsored by the Nuclear Regulatory Commission (NRC) are directed at establishing the credibility of ISI and identifying the requirements necessary to assure light water reactor (LWR) operating safety.

\subsection{OBJECTIVES}

This document reports the first phase of a five-year program entitied "Integration of Nondestructive Evaluation Reliability and Fracture Mechanics." The primary objectives of this program are as follows:

1. Determine the reliability of ultrasonic ISI performed on commercial LWR primary piping systems.

2. Using fracture mechanics analysis, determine the impact of nondestructive evaluation (NDE) unreliability on system safety and determine the level of inspection reliability required to assure a suitably low probability of piping failure.

3. Evaluate the degree of reliability improvement that could be achieved using improved and advanced NDE techniques.

4. Based on material, service, and NDE uncertainties, formulate recommended revisions to ASME Section XI and regulatory requirements needed to assure a suitably low probability of system failure. 
At the direction of the NRC, the Pacific Northwest Laboratory (PNL) has established a four-phase program to meet these objectives. Phase I, reported here, has the principal objectives of scoping and identifying the limits of ISI reliability, estimating current levels of reliability, and making a preliminary fracture mechanics analysis. Phase II of the program has two primary objectives: 1) measurement of NDE reliability through round-robin inspections and 2) fracture mechanics analysis for the purpose of establishing the required level of NDE reliability as well as the impact of current reliability levels. Phase III will inciude evaluations of improved and advanced inspection techniques which will be required to achieve higher levels of inspection reliability. The principal objective of the Phase IV program is to establish

a unified set of recommendations for modifications of existing code and regulatory requirements necessary to achieve a suitably high level of inspection reliability. Phase I was conducted primarily in FY-1979. Phase II will be completed during FY-1980 and 1981, Phase III during FY-1981 and 1982, and Phase IV activities primarily during FY-1982 and 1983. Recommendations for improved inspection requirements will be made throughout the program as data become available.

U1trasonic inspection is the principal inspection technique employed for ISI of primary piping systems and will be the primary focus of the program; however, other volumetric and surface inspection techniques will be considered as necessary. Failure history (Bush 1980) has shown that flaws of major concern, found in service, are located on the piping inner surface. This study is therefore directed toward detection, sizing, and location of these surfaceconnected defects (primarily cracks) with limited concern for internal fabrication flaws. Detection is considered to be the key element in the inspection process.

\subsection{TECHNICAL APPROACH}

The technical approach for this program is directed toward the formulation and implementation of recommendations to be made during the course of the program. To assure acceptance of the recommendations the following action steps will be followed: 
I. Identify the problem areas.

2. Demonstrate the impact on inspection reliability.

3. Define the impact of the inspection unreliability on system safety using fracture mechanics analysis.

4. Formulate recommendations for resolution of the problem.

5. Demonstrate the effectiveness of the recommendations and the resultant gain in confidence in system safety.

6. Evaluate the impact of implementing the recommendations.

7. Assist in implementation of the recommendations.

Demonstrations and evaluations will use materials and welds typical of primary piping systems. Test conditions and variables will be fixed at realistic but conservative values throughout the program. Particular attention will be given to the source and magnitude of the various errors that contribute to inspection unreliability.

There is little constructive benefit in performing a test such as a round robin to demonstrate only that current practices are ineffective. Tests performed under this program will demonstrate the effectiveness of minimum code requirements, as-practiced field procedures, and improved techniques. The tests witl also identify the various sources of error and their impacts on inspection reliability. After the sources of error are identified, it will be possible to formulate appropriate techniques and code requirement revisions necessary to achieve the required inspection reliability.

\subsection{REPORT ORGANIZATION}

This report is divided into seven sections. The introduction and summary are Sections 1 and 2, respectively. Section 3 describes the results of an ISI state-of-practice survey. Section 4 deals with the fracture mechanics and critical flaw sizes for primary system piping. The statistical design and analysis of the experiment are discussed and the Phase II round robin is described in Section 5. Section 6 describes the sample cracking process developed to 
create defects in test samples. The results of the Phase I measurement and evaluation programs as well as estimates of the current levels of inspection reliability are reported in Section 7 . 


\subsection{SUMMARY}

The Phase I program was conducted over the period from October 1978 through February 1980. The primary conclusion resulting from Phase I investigations is that the current inspection requirements of the ASME Section XI Code are inadequate for the detection and reporting of unacceptably large flaws. Specific results and conclusions from each of the five tasks in Phase 1 are sumnarized below.

\subsection{STATE-0F-PRACTIVE REVIEW (SECTION 3)}

A survey was conducted to determine the state of practice of ultrasonic ISI of LWR primary system piping. This review was conducted to define the actual procedures and practices that are applied in the field. Four utilities, five inspection organizations, and three domestic reactor manufacturers were interviewed.

The principal findings of this review are:

1. Test procedures are very similar and are in general designed to meet on ly minimum code requirements. Supplemental tests (after an indication has been located) are substantially different.

2. Selection of search units is highly variable.

3. Only one organization measures and periodically verifies the operating characteristics of their search units.

4. Confidence in the ability to detect defects was highest for ferritic piping and lowest for cast stainless steel.

5. Recording of geometrical indications during baseline preservice inspection (PSI) or ISI is not consistent and, therefore, of limited value.

6. Nominal and worst-case counterbore conditions described by the inspectors provide an insight into the inspection difficulties produced by this condition. 
A summary of the state-of-practice review is described in Section 3 . The complete review will be published as a separate document.

\section{2.? FRACTURE MECHANICS ANALYSIS (SECTION 4)}

Fracture mechanics analyses were performed for the purpose of establishing required NDE sensitivities. Critical flaw size estimates were made using: ASME Code allowable stresses and the methodology of Section XI, the dual criteria of linear elastic and net section plastic collapse for bounding of the critical crack size, and the concept of tearing instability. Crack growth rates were estimated from available literature.

The available analyses of the growth and stability of fiaws in large piping have led to the following results and conclusions about crack failure mode and critical fiaw size:

Failure Mode

1. Evaluations of the consequences of inner diameter (ID) surface flaws in piping indicate that flaws will produce a leak before they will cause a pipe break (Mayfield et al. 1980). Therefore, the definition of critical flaw sizes on the basis of unstable crack growth and fracture toughness considerations is of limited usefulness for application to reactor piping systeris.

2. The failure mode of concern is an increase in crack growth rate such that an ID surface flaw breaks through the pipe wall during the time span between inspections.

3. In accordance with the leak-before-break condition, critical defects for unstable crack growth have been found to be through-wall flaws of significant length. For axial flaws the length is about half a pipe diameter, and for circumferential flaws the length is about half the circumference of the pipe.

Variation of Critical Flaw Sizes

1. Evaluations of crack growth rates show significant differences in allowable defect sizes both from pipe to pipe and from location to location within a given pipe. 
2. Even under conservative assumptions (e.g., levels of vibrational stress, fatigue crack growth rates, and threshold $\mathrm{K}$ values) many welds can tolerate quarter-wall defects and achieve a 40-year design life (Mayfield et al. 1980).

3. Conservative analysis methods indicate a finite probability of failure at certain locations (particularly at dissimilar metal welds) within one percent or less of design life for flaw depths as small as one tenth of the pipe wall thickness.

4. Nevertheless, the overall trend of the results suggests a relatively low probability of failure within a 10-year inspection interval (say less than one percent) for flaws of depths less than one fourth of the pipe wall thickness.

\subsection{STATISTICAL OESIGN AND ANALYSIS (SECTION 5)}

The principal objective of the statistical design and analysis task is the formulation of techniques for evaluating the effectiveness of ultrasonic ISI and for designing the test matrix to be used in the Phase II round robin inspections.

Procedures and analysis techniques have been developed to describe and test the effectiveness of ultrasonic ISI performed to the requirements of Section XI of the ASME Code. Probabilities of recording, detection or identification, and rejection will be used. Similar probabilities for false calls in materials without defects will also be analyzed because of the high probability of geometrical reflectors which normally occur during ISI.

The round robin test matrix has been designed to evaluate the effectiveness of field inspection procedures and a recommended improved procedure. The effectiveness of minimum code requirements will also be evaluated. The tests will be performed under conditions which simulate difficult field access conditions. The effect of single-side access conditions will also be evaluated. The size of the test matrix has been selected to provide sufficient measurements to insure reasonable confidence levels for the measurements. The test 
Inatrix requires 96 samples, 6 inspection teams, and 1470 measurements. It is expected that the round robin tests will be initiated in January 1981 and completed by June 1981 .

\subsection{SAMPLE PREPARATION (SECTION 6)}

The objective of this task is the fabricatior of samples with flaws (cracks) artificially induced under controlled corlditions. These cracks are intended to simulate service-induced flaws. These samples will be used to correlate ultrasonic measurments with known crack properties. The cracking methods which have been developed will also be used for fabrication of samples to be used in the Phase II round robin tests.

A thermal fatigue process has been developed for producing realistic cracks in the round robin test samples. This process is capable of producing cracks of controlled length and depth. The tightress and roughness of these cracks represent conservative values compared to those expected in service. The thermal fatigue process is adaptable to complex shapes that would be extremely difficult to fatigue by conventional techniques.

\subsection{MEASUREMENT AND EVALUATION (SECTION 7)}

The primary objective of the Phase I measurement and evaluation task is to identify, measure, and evaluate the impact of inspection variables that limit the effectiveness of ultrasonic in-service inspection of primary system piping. Recommendations for more effective code and regulatory requirements will be based on these measurements and evaluations.

Measurements have been accomplished for the evaluation of the influence of crack size, shape, orientation, roughness, and tightness. Also evaluated were the effects of search unit selection, search unit and instrument operating characteristics, and calibration reflector or inspection sensitivity and reliability.

The principal conclusions of the Phase I measurement and evaluation program is that inspection requirements of the ASME Section XI code are inadequate for the detection and reporting of unacceptably large flaws. This lack of sensitivity and inspection reliability stems from the following: 
1. The calibration requirements of ASME Section XI are inadequate to assure reporting of even ideal reflectors of unacceptable size.

2. Real defects (cracks) will diways exhibit lower reflected signal amplitudes than ideal artificial reflectors because of the influences of crack tightness, roughness, and orientation. Even large cracks (greater than $50 \%$ of pipe thickness) may be "unreportable" according to Section XI criteria.

3. Lack of search unit selection controls can result in highly variable test sensitivity.

4. Lack of controls on measurements of search unit and instrument operating characteristics may result in highly variable test results and a lack of inspection repeatability.

These conditions, coupled witn expected variability due to the inspectors, can result in ineffective in-service inspections. Estimates of current inspection reliability are also made in Section 7 of this report. These estimates predict a very low effectiveness for the $100 \%$ DAC reporting level as presentiy required by ASME. Section XI Code but predict substantial improvements for $50 \%$ and $20 \%$ DAC reporting levels. These estimates agree with the results of an extensive round robin test on lack of penetration and lack of fusion-type defects reported by forli (1979). 



\subsection{STATE-OF-PRACTICE REVIEW OF ULTRASONIC IN-SERVICE INSPECTION OF PRIMARY SYSTEM PIPING}

Early in the program, it was recognized that there was a lack of information regarding the procedures practiced by organizations performing in-service inspections of primary system piping. Research staff at the Pacific Northwest Laboratory (PNL) felt that inspection procedures varied and that the procedures should be documented for reference and for guidance in future research. Several utilities, private inspection organizations, and domestic reactor manufacturers were interviewed. A summary of this survey is included below. The complete review of the state of practice will be published as a separate document.

\subsection{OBJECTIVES AND DEF INITIONS}

The first objective of the review is to define current ultrasonic inspection technology as practiced in the field, including procedures and practices used for the required code volumetric examination of primary system piping and components in commercial LWRs. Section XI of the ASME code defines the inservice inspection requirements of nuclear power plant components. A second objective is to define a combination of test procedure and equipment that is most typical of field practice and that could be used as a basis for determining the capability and reliability of current ultrasonic inspection practices for detecting and sizing flaws in primary system piping.

The state of practice, the technology which is commonly applied in the field, is defined through literature review, surveys, and personal interviews. The state of the art is (by our definition) the technology that has been proven in use and is generally accepted by inspectors but not necessarily by codewriting organizations. A third category is advanced technology, techniques that are under laboratory study but which have not been demonstrated in the field.

This report discusses the information received as a result of personal interviews with four utilities, five private inspection organizations, and three domestic reactor manufacturers. Together these organizations encompass 
the major portion of domestic in-service inspection experjence in ultrasonic inspection at nuclear power plants. Information furnished to the pacific Vorthwest Laboratory described ultrasonic inspection techniques, test procedures specific to materials examined, equipment and transducers, 5 tandards, confidence limits, and such problem areas as pipe counterbore. These data will be used to define a generic procedure and equipment combination that is most typical of primary piping inspections. This combination of procedures and equipment will then be used in our laboratory investigations as the basis for determining the capabilities and limitations of ultrasonic nondestructive evaluation (NDE) for the detection, sizing, and location of flaws typical of service-induced flaws. Important permutations and combinations of procedures and equipment will also be evaluated. Finally, advanced techniques will be evaluated and compared to the state-of-practice techniques.

\subsection{THE NEED TO DOCUMENT THE STATE OF PRACTICE}

At the onset of this five-year program, uncertainty existed as to what specific techniques, procedures, equipment, transducers, methods of signal evaluation, etc., constituted the current state of practice for ultrasonic weld examination at commercial nuclear power plants. It was our belief that the current state of practice was highly variable and should be documented. Specific to this study, Subsection IWB-2500 of Section XI Categories B-F and $3-J$ of ASME Code defines those areas subject to examinations and the extent and frequency of examinations for class 1 pressure-retaining welds. Subsection IWB-2500 requires volumetric examination of these inportant class 1 pipe welds with ultrasonic inspection being the preferred method of examination. Figure 3.1 shows a cross section of a typical welded pipe joint.

Early results from personal interviews showed that differences exist in inspection techniques, equipment and transducers, personnel training, standards, experience per inspection of particular material, test confidence, experience with inner and outer diameter geometry conditions, data recording methods, evaluation criteria, testing philosophy, and use of supplemental nondestructive testing techniques such as radiography. 


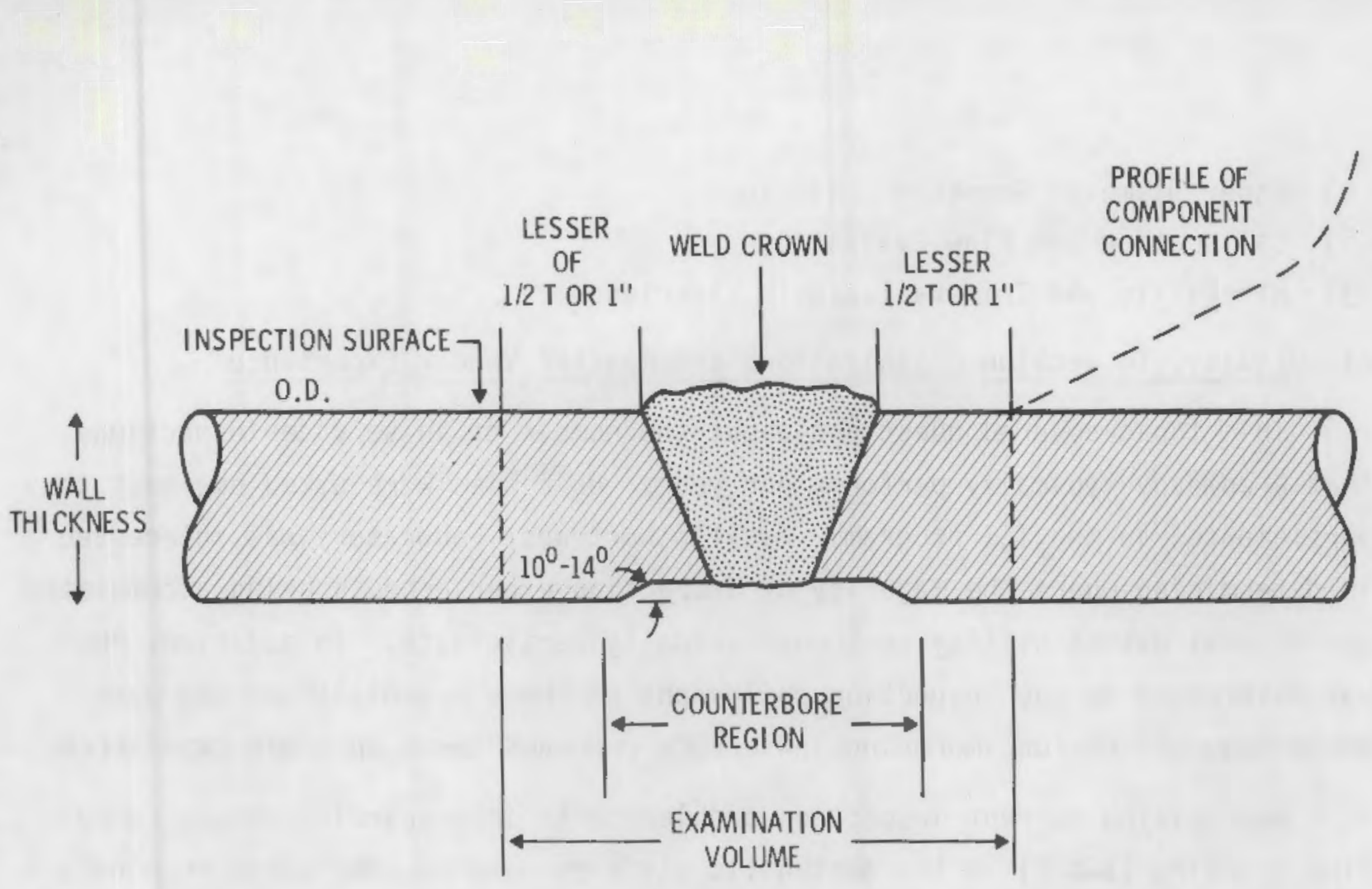

FIGURE 3.1. Cross Section of a Typical Welded Pipe Joint

Our preliminary findings are summarized by topic in the following paragraphs. Some topics need further discussion and verification so that information will not be misconstrued. A final report will detail and summarize the responses of the utilities, inspection organizations, and reactor manufacturers.

\subsection{STATE-OF-PRACTICE SURVEY RESULTS}

The interviews covered 10 topics specific to ultrasonic in-service inspection:

a) Utility, Inspection Organization, and Reactor Vendor Experience

b) Selection and Performance Verification of Transducers

c) Manual Piping Inspection Equipment

d) Inspection Practices for Ferritic and Stainiess Steel Pipe

e) Experience with Centrifugally Cast Stainless Steel Pipe

f) Automated Inspection Experience

g) Outer Diameter Geometry Conditions 
h) Inner Diameter Geometry Conditions

i) Calibration and Flaw Evaluation

j) Bimetallic and Trimetallic Weld Experience.

a) Utility, Inspection Organization, and Reactor Vendor Experience

This topic covered questions about the number of in-service inspections that a company typically performs per year. Utilities were asked how much they participated in the ISI program. Pacific Northwest Laboratory was interested in determining where the majority of inspection experience is being accumulated and to what extent utility personnel actually participate. In addition, PNL was interested in the inspection philosophy of these organizations and possible suggestions for improving in-service programs based on their experience.

Recognizing current inspection problems with intergranular stress corrosion cracking (IGSCC) in the austenitic stainless steels, PNL asked respondents about the number of inspections in which they had participated, looking specifically for IGSCC. Respondents were also asked about their experience and confidence in the inspection of centrifugally cast stainless steel (ccSS) welds. The large grain structure characteristic of CCSS creates uncertainty in the inspectability of CCSS welds. Responses to these questions are detailed under e) Experience with Centrifugally Cast Stainless Steel Pipe.

To date there have not been any cracks found in the primary reactor coolant piping at domestic pressurized-water reactors (PWRs). Private ISI organizations and reactor vendor organizations perform about $95 \%$ of the domestic ISI programs at commercial nuclear power plants, of which there are currently $\sim 63$ operating units. Utility personnel generally plan and oversee the ISI program with inspections by utilities themselves generally limited to special problem areas. However, the trend at some of the larger nuclear utilities is to increase utility inspection staff, thereby improving in-house inspection capability in addition to having broader versatility to plan, conduct, and execute their in-service inspection, testing, and maintenance programs. This appears to be a very cost effective approach for these larger nuclear utilities.

The survey respondents had different levels of specific inspection experience with IGSCC. Because of the incidence of IGSCC at particular plants 
throughout the U.S., the experience of utility personnel at these affected plants appears substantial. By comparison, one respondent from an inspection organization that participates in 30 to 40 ISI programs yearly stated that, to his knowledge, their people were not involved with any inspections looking specifically for IGSCC. Another respondent indicated that his company had been contracted specifically to look for IGSCC in certain piping systems at four plants; although he found no strong evidence of IGSCC, there was uncertainty at two plants concerning uitrasonic indications in the sensitized area of some welds. Respondents' answers also indicated differences in IGSCC test procedures, examination techniques, transducers, data recording leveis, training, and standards. These differences are discussed further under d) Inspection Practices for Ferritic and Stainless Steel Pipe.

The Electric Power Research institute and the U.S. Nuclear Regulatory Commission are funding several research programs concerning IGSCC in piping systems.

\section{b) Selection and Performance Verification of Transducers}

Several studies (Buchanan 1976; ASNT 1977; Serabian and Lawrie 1977; Birks and Lawrie 1979) have shown the dramatic variability in measurement accuracy and repeatability that result when transducers are not properly characterized but $r$ andomly selected for use. This topic covered questions about the criteria for selecting transducers and the methods used to verify the operation and performance of transducers. Respondents were asked if certain parameters (such as center frequency, radio frequency (RF), waveform, sensitivity, or frequency bandwidth) were routinely measured to verify transducer performance. Respondents were also asked about methods used to periodically check transducer performance. Numerous technical studies now under way under the auspices of the U.S. Naval Research Laboratory, National Bureau of Standards, ASTM, PVRC committees, and other organizations are emphasizing the development of appropriate specifications and testing procedures for the evaluation of ultrasonic system components.

Inspectors select transducers based on inspection experience and the demands of a given test procedure. A typical test procedure states the nominal 
operating frequency and transducer size range that can be used. For shear wave examination, beam spread and exit point are measured.

In order to verify initial transducer performance, most companies request manufacturer's certification (RF waveform and frequency spectrum) and the results of a standard calibration. Center frequency is generally assumed from manufacturer's certification; two respondents check center frequency themselves, and one respondent checks the beam profile. Others felt that taking a beam profile has little merit.

The industry makes no checks of near-surface resolution. One company checks transducers on an inner-diameter reflector (notch) if they are going to inspect thin-wall pipe. Calibration blocks with side-drilled holes (SOH) are used to calibrate for distance amplitude correction (DAC) and to establish a sensitivity level. Resolution is checked using SOH. Almost half the respondents use International Institute of Welding (IIW) blocks for verification of beam angle and exit point. One respondent uses an IIW design modified to closer tolerances $\left(1^{\circ}\right.$ measurements added).

Records of transducer certification are often kept, and all DAC curves are recorded and documented as required by test procedures. The philosophy of several respondents was that a successful DAC calibration (within a few decibels of typical values for a specific pipe standard) indicates that the transducer is good. Any need for a significant increase in gain to maintain a given DAC curve is often perceived as a sign of transducer problems. Only one respondent indicated the use of periodic (biannual) checks on transducer performance (RF waveform frequency spectrum and distance-versus-amplitude plot).

Al1 respondents estimated that the typical lifetime of an angle-beam transducer is two to three years for inspection organizations. Special units last up to five years.

c) Manual Piping Inspection System

This series of questions was intended to identify the ultrasonic flaw detection instruments used in practice: the make, model, modifications (if any), and reasons for modifications. The use of electronic DAC, external 
recording devices, requirements on warm-up time, and periodic calibration or measurement requirements not specified by Code were also covered in the survey.

Although companies use many different instruments for manual piping inspection, the most common is a Sonic Mark I. The Nortec 1310, Krautkramer USIP II, and Branson 301 and 303 instruments are also widely used. These instruments are usually unmodified with no particular requirements other than manufacturer specifications for instrument pulser or receiver bandwidth. Electronic DAC is not used for manual pipe inspection. Only one respondent Ises an external recording device (a strip chart) to record all analog data for each weld inspection.

Standard practice is to completely wrap the instrument in a plastic bag to protect it from contamination. The bag is taped, and the instrumenttransducer system is then calibrated. The most common practice is to recalibrate the instrument before it is removed from the containment area and decontaminated. All respondents indicated that wrapping the instrument does not alter instrument performance. However, there was concern about instability problems that occur in containment at temperatures of $125^{\circ} \mathrm{F}$ and higher.

Less than half of the respondents apply periodic calibration or measurement requirements more frequently than specified by code.

The requirements for instrument warm-up time vary from no warm-up to thirty minutes for manual systems and one hour for automated systems. Although several companies have no requirements for warm-up time, the instrument is usually on two to five minutes before the operators are ready to begin inspection. Two companies that recommended a specific time for instrument warm-up indicated that their test procedure did not call for it. One respondent observed a three-decibel difference between instrument calibration after warmup and calibration immediately after the instrument is turned on. A few companies noted problems with instrument stability that were related to instrument warm-up time, battery charge, or line voltage fluctuation. One respondent noted that portable instruments are generally sensitive to line voltage and temperature chanqes. Another respondent found line voltage fluctuations to be a problem when a generator was used for automatic inspection at some 
sites; he also found that using new batteries for each major inspection alleviates problems that occur with battery-powered units.

d) Inspection Practices for Ferritic and Stainless Steel Pipe

With this topic, PNL intended to identify the specific transducers and any special procedures used for testing and detecting cracks in ferritic steel and austenitic stainless steel (SS) piping. Respondents were asked to indicate what confidence they had in detecting cracks.

The industry uses tranducers of many different sizes with transducer selection dependent on wall thickness--generally the thicker the wall, the larger the transducer. Typically, minimum Code inspection requirements are performed: on ferritic pipe, a $45^{\circ}$ shear wave examination at $2.25 \mathrm{MHz}$. Standard Code procedures are used without exception; no special procedures are used. Inspection confidence with ferritic pipe was very high.

Many respondents use the same transducers for austenitic SS pipe as they use for ferritic pipe: $2.25-\mathrm{MHz}$, single-element transducers in $45^{\circ}$ shearwave examinations. Several companies use transducers of the same size but with a 1.5-MHz nominal frequency. Special test procedures with more extensive examination techniques are required by some companies. These procedures and examination techniques are undergoing review.

All respondents have evaluated or have experience in using a dual-element transducer for inspection of austenitic SS pipe. The reported advantages of the dual-element transducer are "less noise," "cleaner recording," and "seems to work well with thin material." Other respondents are considering use of the dual-element units but have no specific plans for using them.

Most companies use the same pipe test standards for IGSCC inspection of austenitic SS pipe as they use for ferritic pipe (i.e., 10\% T notch) per ASME Section XI, Appendix III. Two companies use a 5\% T notch standard, and one company uses actual IGSCC pipe samples. Several respondents indicated that it would be beneficial to make available to inspection personnel the many IGSCC samples currently stored at Battelle Columbus Laboratories. Several companies concerned with ultrasonic examination of pipe welds for IGSCC have developed written procedures similar to those documented by Serabian and Lawrie (1977). 
Confidence in detecting IGSCC in austenitic SS with depths from $10 \%$ to' $25 \%$ of wall thickness ( $T$ ) ranged between $50 \%$ to $85 \%$. One respondent indicated that confidence in detecting cracks at 10\% T is "borderline." At 25\% to 50\% T inspection confidence averaged about $78 \%$. At greater than $50 \% \mathrm{~T}$, inspection confidence was calculated to be $95 \%$. These confidence estimates are based on the experience of the individual respondents and have little statistical significance; however, they indicate the relative degree of difficulty associated with detection of flaws in austenitic SS compared to ferritic material.

e) Experience with Centrifugally Cast Stainless Steel Pipe

Respondents were asked several questions concerning their experience with the inspection of CCSS pipe. Use of specific transducers, procedures, instruments, standards, and test confidence estimates were discussed.

Respondents estimated that 3 to 10 domestic plants contain primary reactor coolant piping of CCSS. At least half of the respondents indicated some experience with CCSS pipe. The preferred method of examination is with a 1.0- or 1.5-MHz single-element or dual-element transducer using a refracted longitudinal wave incident at $40^{\circ}$ to $45^{\circ}$ Standard field flaw detector instruments are used in conducting the examination. Because of the characteristically large, finger-like grains of this material (see Figure 3.2), examination of the weld using $45^{\circ}$ refracted shear waves is impractical.

Most of the inspection experience with CCSS seems to be with one inspection organization. The respondent from the experienced inspection organization felt confident that he could detect any significant flaws using a $40^{\circ}$ to $45^{\circ}$ refracted longitudinal technique. Other respondents with experience in inspection of this material were more pessimistic about the detection capability and reliability of this ultrasonic inspection technique but showed a wide range of inspection-detection confidence. Some specific comments were: "difficuit", "not very confident in test", "good-fair", "pretty good", "can see code-size reflectors; however, there are many metallurgical reflectors in this cast material".

Current standards are pipe sections with $5 \% \mathrm{~T}$ Code notches and/or sidedrilled holes. Several respondents indicated the need for better standards. 
ก

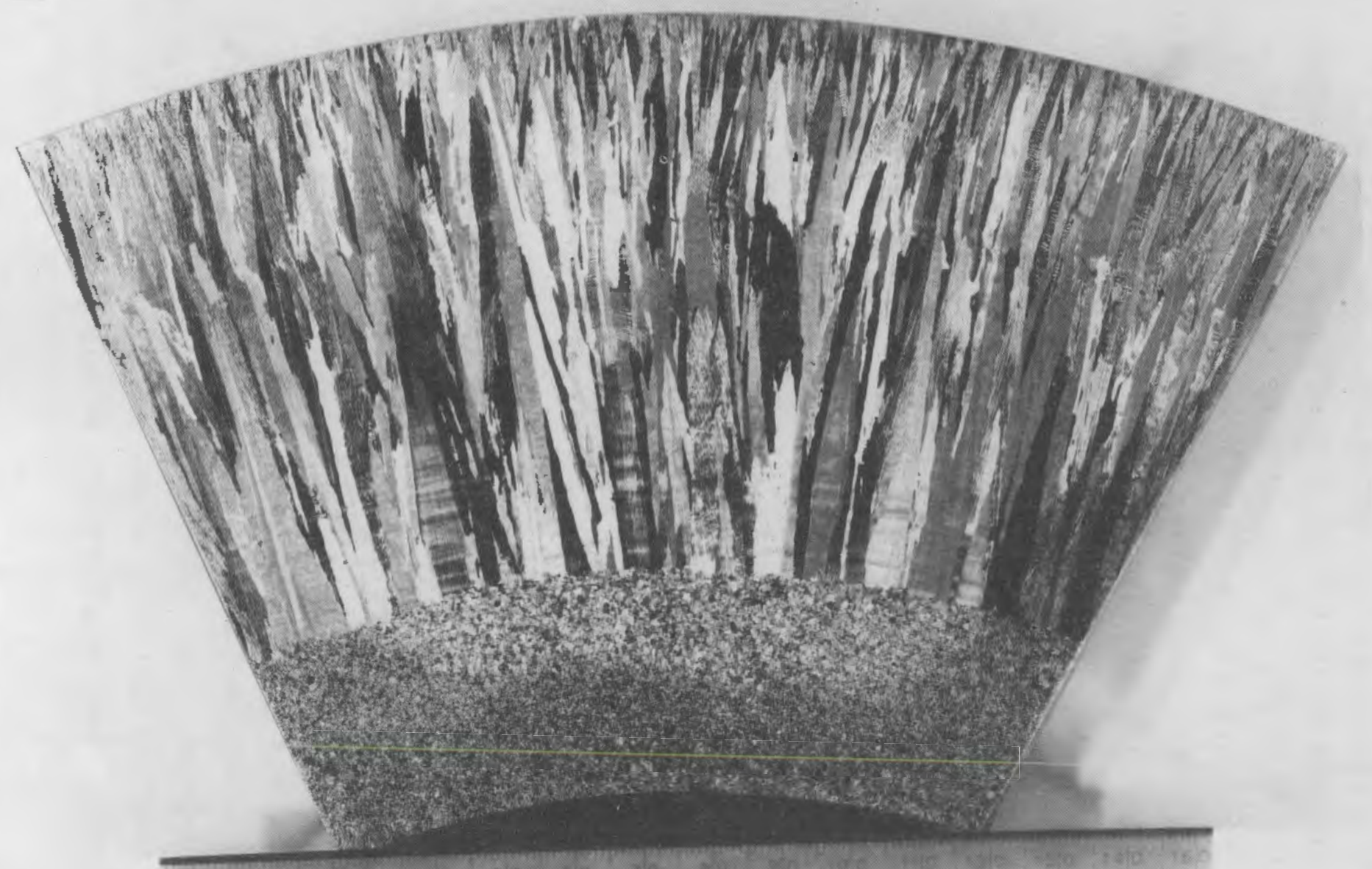

FIGURE 3.2. Pipe Cross Section of Centrifugally Cast Stainless Steel 
All respondents agreed that there is a need for additional study on inspection practices for CCSS pipe, and one reactor vendor is conducting a substantial prograin on the inspection of CCSS pipe.

\section{f) Automatic Inspection Practice}

This topic details the industry use of automated uitrasonic inspection systems: frequency of use, estimates of relative efficiency, data recording standards, couplants used, and special requirements.

Most respondents had used automated inspection systems but on y to inspect nozzle-to-safe-end and safe-end-to-pipe welds. Respondents indicated that automated inspection accounts for $5 \%$ to $25 \%$ of the inspection program. The respondent who quoted a $25 \%$ use of autiomated systems inspection pointed out that this is only used during the preservice (baseline) inspection program.

All respondents stated that manual inspection is superior to automatic inspection. Manual inspection is always preferred for critical problems. One respondent criticized the equipment design. Automated systems are unproven, require specific access conditions, and seem to break down at the most inappropriate times. Automated systens typically require as much time as manual systems (or more) to set up, calibrate, and conduct an inspection. Automated systems are less versatile but do offer improvements in measurement repeatability.

No industry standard exists for recording automatic inspection data. Strip charts, analog magnetic tape recording, analog-to-digital conversion with magnetic tape recording, and videotapes are current methods of recording uitrasonic data.

Water is the sound couplant for these automated systems. Systems are calibrated statically using pipe standards supplied by the utility, and then placed on the pipe for the dynamic inspection of the welds.

Weid preparation is important when employing automated systems. In practice these systems do require certain access limits. Typical access requirements (e.g., safe-end) are $18 \mathrm{in.}$ on either side of the weld. Weld crown 
height should be flush or have a smooth taper. More complete data describing currently used systems and others under development will be reported in the final state-of-practice report.

\section{g) Outer Diameter Geometry Conditions}

The survey discussion of pipe outer diameter (00) geometry included information on single-side access, weld crown height, diametrical shrink, surface finish, undercut, and pipe-to-component 00 conditions.

Respondents estimated that typically $20 \%$ of the welds for primary reactor coolant piping have single-side inspection access. However, one company replied that $90 \%$ of the welds for their PWR units have single-side access and $60 \%$ to $70 \%$ of the welds for their boiling water reactors (BWRs) have singleside access. Further, a reactor vendor spokesman stated that $90 \%$ to $100 \%$ of the primary reactor coolant system welds have single-side access. Respondents indicated that single-side access results because of pipe-to-valve and pipeto-elbow conditions. Most respondents indicated no special test procedures exist for single-side access inspection; they can usually get a $1 / 2 \mathrm{~V}$ examination in most cases, or a $3 / 2 \mathrm{~V}$ root examination when $1 / 2 \mathrm{~V}$ examination is impossible. Full $V$ examination is used for inspection of the upper weld crown area.

Respondents estimated that essentially $80 \%$ to $100 \%$ of the primary reactor coolant system welds were ground. New plants under construction have welds that are blended. 01der operating PWR and BWR plants have a variety of conditions; the most common reported condition is with the crown ground flat with an edge height of $0.03 \mathrm{in}$.

In general, there are no special procedures used to compensate for severe diametrical shrinkage. Respondents indicate that shrinkage is usually not a problem in thick-wall pipe, but occurs more often in thin-wall pipe.

Respondents indicate that pipe surface finish is typically of good quality (e.g., 125 to 250 rims). 


\section{h) Inner Oiameter Geometry Conditions}

The survey discussion of pipe inner diameter (10) geometry included information on pipe counterbore conditions and problems resulting from counterbore geometry.

Figure 3.3 indicates the range of welded pipe counterbore conditions as sketched by the survey respondents. These sketches represent estimated worstcase and nominal-case conditions. As-built information on the counterbore is rarely available to the surveyed companies. Respondents indicated that design specification drawings are usually available, but they question the usefulness of this information because of the optional methods currentiy allowed for pipe joint fabrication at commercial plants. In older operating plants, the design specification drawings are often unavailable.

In general, the survey respondents felt that their capability for accurately measuring counterbore geometry ranged from "poor" to "very poor." Most companies use a single-element transducer with a $0^{\circ}$ examination (thickness measurement) at $2.25 \mathrm{MHz}$ to $5 \mathrm{MHz}$. However, one respondent used a dual-eiement, line-focused transducer. Some occasionally use radiography when possible.

The availability of baseline information on geometric reflectors varies from "not very often" to "40\% to $50 \%$ of the time" to "frequently available." Respondents indicated that the baseline information on geometric reflectors is generally correct. However, in one case where destructive analys is had been performed, one respondent reported that stress corrosion cracking was labeled as a geometric reflector. Counterbore geometry, weld root suck-up, and weld root drop-through were mentioned as key pipe ID concerns. One respondent indicated that sometimes geometric indications are reported and sometimes they are not: if indications are not reported on the data sheet but are noted by plant inspection personne1, the reporting of the geometric indication in future inservice inspections is used as a check on the inspector.

Respondents were asked if they thought there was a need for a standard format for recording geometric indications. Severa? see no need for a standard format for recording geometric indications. Other respondents believed that a standard format is needed and that it should be a Code requirement. 


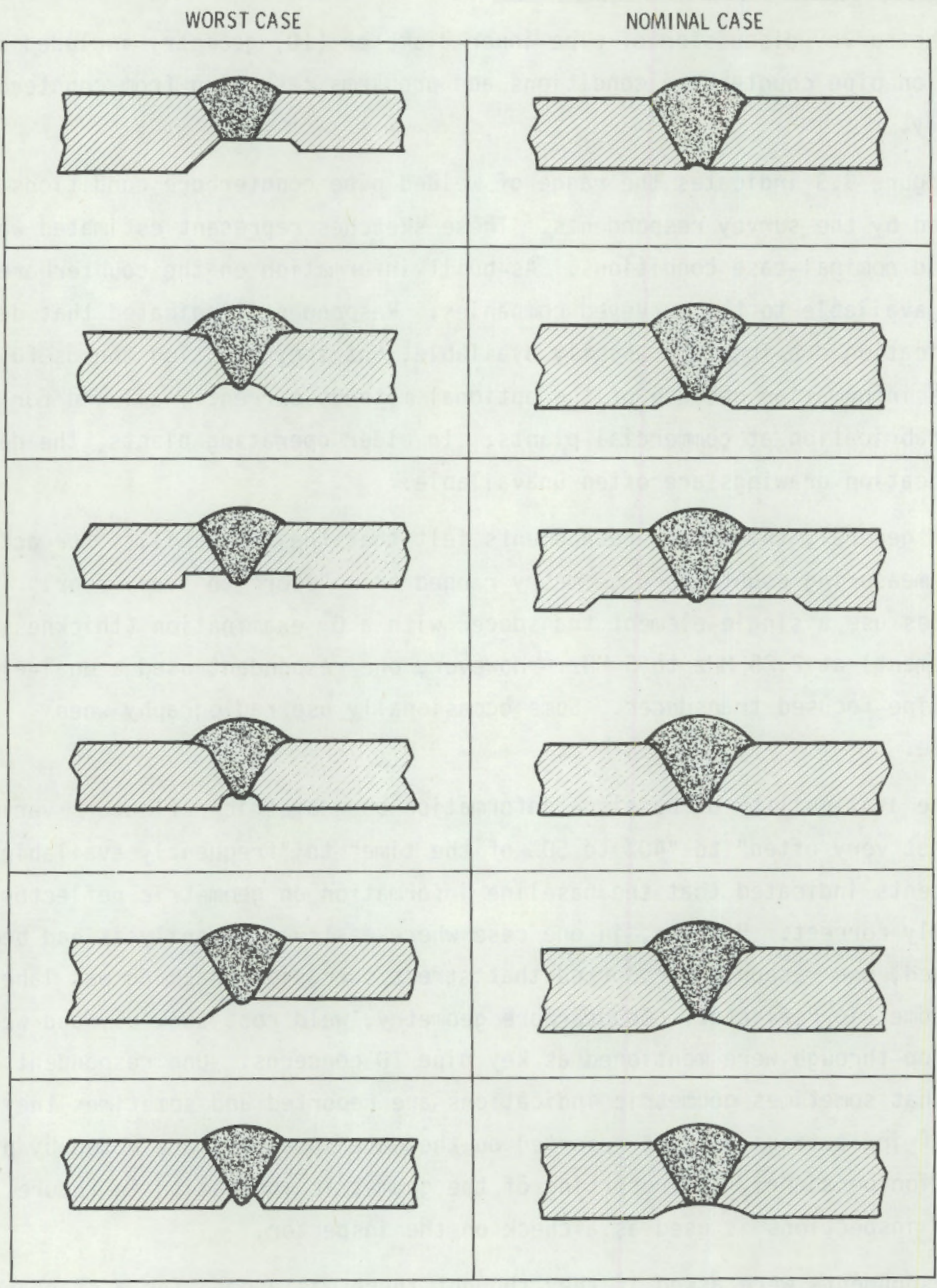

FIGURE 3.3. Pipe Counterbore Geometry Conditions 
Those respondents that did not feel the need for a standard fornat stated that the recording of geometric reflectors is essentially a code requirement: the code specifies recording of all defect-like indications which exceed the reference level. One respondent fell that all tests snould be strip chart recorded for a permanent record of each examination.

Opinions varied concerning the value of bas 21 ine data gathered by different organizations. Responses ranged from "it should be valid i" procedures and calibration blocks are the same" to "often very little value, since levals of recording and test procedure can be different" to "do not record geonetry reflector for basaline."

Respondents were asked how they evaluate $3 / 2 \mathrm{~V}$ indications when the bean is incident on the conical section of the counterbore. Respondents indicated that trey can get a $1 / 2 \mathrm{~V}$ examination of the weld root for the majority of primary system welds.

i) Calioration, Inspection, and Flaw Evaluation

Calibration verification and inspection practices were discussed in the survey, and a series of questions were asked concerning flaw evaluation and recording methods.

Typical industry practice is to verify initial instrument caliaration by recalibrating at least every four hours and/or at every operator change. Scan overlap during calibration/inspection is $10 \%$ to $25 \%$. Some skew is isually used during calibration/inspection, but the amount is neither mandatory nor recommended. Companies use a $45^{\circ}$ skew for IGSCC inspection. The skew for ferritic pipe inspection is usualty $15^{\circ}$ to $20^{\circ}$.

In-practice flaw evaluation and recording methods were probed witi the questions indicated in the following paragraphs.

\section{What is the data recording procedure?}

Most respondents record signal indications that are $50 \%$ of $0 A C$. Several respondents investigate and note signals $20 \%$ and $25 \%$ of DAC. One prucedure called for the recording of indications down to $20 \%$ of the maximum indicated amplitude. 
2. What is the position accuracy on the pipe surface?

Estimated positional accuracy on the pipe surface during inspection varied from 0.02 to $0.2 \mathrm{in}$.

3. What is the time metal path accuracy for $1 / 2 \mathrm{~V}$, and $3 / 2 \mathrm{~V}$ inspections?

Estimates of time metal path accuracy for $1 / 2 \mathrm{~V}$ examination were as small as $0.01 \mathrm{in}$. and as large as $0.125 \mathrm{in}$. Estimates of time metal path accuracy for $3 / 2 \vee$ examination ranged from 0.02 to $0.2 \mathrm{in}$.

4. Are there supplemental tests? Other angles, frequencies? Two-side evaluat jon? Finger damping?

Standard practice is to use a $45^{\circ}$ examination of each side of the weld. When a flaw is suspect, companies use $60^{\circ}$ and $70^{\circ}$ supplemental examinations. Finger damping is a common practice used to check on extraneous signats.

5. What methods do you use to call ID geometry? o0 geometry?

For 10 geometry, inspectors use reflector position i i.e., proximity to weld root) and the extent of indication (e.g., a $360^{\circ}$ single reflector often indicates geometry conditions). Signal shape and characteristics, piotting methods, and refracted angle conversion charts are also used. Inspectors use many of these techniques to determine 00 geometry. Finger damping is a key test for 00 geometry.

6. When and how often do you call for radiography? How useful is radiography?

Inspectors call for radiography if they think that the ultrasonic test is inadequate for making a reliable inspection. The utility usually requests a radiograph if a flaw is called. One respondent calls for radiography if other radiographs are already on file (for comparison). Another calls for radiography if the inspector suspects IGSCC, which was estimated to occur about once per site inspection. The radiographs may then show weld spatter or can sometimes detect IGSCC. One inspector noted that radiography is not usually used for in-service inspection, while 
others indicated that radiography is used quite often. Most respondents judged radiography to be useful in some cases, but they varied in their estimate of its effectiveness.

\section{When do you call for penetrant?}

Most inspectors call for penetrant when ultrasonic inspection indicates suspect surface anomalies or through-wall indications. One company calls for penetrant when the weld has only single-side access.

8. What supplemental information do you call for and what is your confidence in them?

A variety of supplemental information is used but not necessarily all the time: design drawings, radiograpinic testing records, welding procedures, weld specifications, weld preparation data, previous ultrasonic inspection data, contour gauge measurements, as-built drawings, or other information may be available. The consensus seems to be that such information can be helpful aithough its reliability must be suspect, especially with older plants.

\section{j) Experience with Bimetallic and Trimetallic welds}

Respondents were asked about their experience with bimetali ic and trimetallic welds. Companies indicated that the bimetallic (e.g., carbon to stainless) and trimetaliic welds (e.g., carbon to Inconel to stainiess) produce many indications (particularly along the fusion zone interface) that complicate ultrasonic examination. Respondents try to use the most attenuative material for standards, but standards vary. One respondent indicated that it is not uncommon to experience a 20 - or 30-decibel loss in signal amplitude when looking through the weid zone at known artificial reflectors in the heat-affected zone of the weld. Training with dissimilar metal weld standards is a common practice. Test confidence in detection of flaws was estimated at 50 to $60 \%$.

The Electric Power Research Institute is funding a research program investigating bimetallic and trimetallic weld inspection.

\subsection{STATE-OF-PRACTICE SUMMARY AND CONCLUSIONS}

The uitrasonic examination procedures used for in-service inspection and evaluation of class 1 primary reactor coolant system welds are essentially the 
minimum requirements documented in the 1974 edition of the ASME Code, Section XI. Special ultrasonic examination procedures for the detection of intergranular stress corrosion cracking and flaws in centrifugally cast stainless steel are more variable. Major differences occur in the examination techniques, the methods and procedures used for evaluating indications after detection, and the method and extent of data recording.

A major difference of opinion exists as to the need for recording geometric reflectors during preservice inspection (PS:). Some organizations do not record geometric indications during PSI. When geometric indications are recorded, their usefulness for ISI is sometimes questioned because there is no standard format for data recording. Researchers at. PNL beliave that a welldocumented PSI would substantially improve the raliability of in-service inspections.

A wide range of nominal and worst-case counterbore geometry conditions were reported. Many of these conditions are unacceptable according to Section III of the ASME Code and could lead to highly variable inspection results. As-built. information on counterbore geometry is seldom available and is difficult to determine by NDE methods.

Only one company performs periodic evaluations on search units used for ISI. Few organizations make any search unit characterization other than the manufacturer's test report (RF waveform and frequency spectrum). Many respondents feel that extensive search unit or instrument characterization has no substantial benefit. Researchers at PNL believe that repeatable test results can be obtained only with well-characterized search units and instruments. Evaluations are in progress to determine the magnitude of these test variables.

The respondents uniformily agree to a high-confidence level (greater than $90 \%$ in their ability to detect flaws in ferritio steel piping. For flaws in austenitic stainless steel piping the confidence estimate is 10 wer--50\% to $35 \%$ for flaws from $10 \%$ to $25 \%$ of through-w 11 tinickness and approximately $90 \%$ for flaws greatar than $25 \%$ of through-wall thickness. Confidence for dissinilar matal weld joints ranges from $50 \%$ to $60 \%$ beca:lse of the high attenuation and spurious reflectors common to these welds. Confidence levels for ccss are highly varjable with one organization expressing a ralatively high confidence 
(due to their independent researcin) compared to others who expressed no confidence. Thesa levels of confidence should not be construed to have any statistical basis because they are personal opinions based on individual experiences. However, these opinions do give a realistic indication of the relative degrees of difficulty associated with inspection of these materials.

Over the last several years the utilities, the U.S. Nuclear Regulatory Commission, the Electric Power Research Institute, and other private nonde. structive testing organizations have been increasingly aware of the need to improve both qualitative and quantitative nondestructive measurement capabilities for inspection of primary piping systems at commercial nuclear power plants. Several of the more difficult areas of pipe weld inspection have been noted here. The review showed that there are noted differences in the aspracticed ultrasonic inspection methods and procedures used at commercial nuclear power plants. Researchers at PNL believe that further documentation of these practices will substantially benefit both NDE practitioners and regulatory personnel by providing technical information that will increase and improve the understanding of current code inspection practices. 



\subsection{FRACTURE MECHANICS ANALYSIS}

The primary objective of the fracture mechanics analysis in this program is to determine the impact of inspection unreliability on system safety; i.e., the consequences of a failure to detect unacceptably large flaws during inservice inspection. A second, longer-term objective is the establishment of inspection requirements necessary to assure a suitably low failure probability after considering the uncertainties of inspection reliability as well as service and material variables. Probabilistic fracture mechanics calculations will be required in establishing these inspection requirements. Duplication of effort with related projects sponsored by NRC and others has been avoided. In particular, results of the cold Leg Integrity Evaluation performed at Battelle-Columbus Laboratories (Mayfield et al. 1980) have been utilized extensively.

Fracture mechanics analyses in this task to date have been of two types. An initial defect sensitivity study evaluated the significance of such factors as defect size, shape, location, and orientation as well as stress state variables such as biaxiality and stress gradients. Details of the sensitivity study are given in Appendix A. The results of the defect sensitivity studies reinforced the significance of ID surface $f$ laws, which are of prime concern since in flaws will initiate and grow at faster rates because of environmental effects. Subsequent estimates of critical flaw sizes therefore focused on IJ surface flaws. The "critical flaw size" is the size at which a crack grows unstably to fracture at a given load. Several alternative approaches were used for critical flaw size estimates which were required to establish sensitivity ranges for NDE. The methods and results are described below.

\subsection{RESULTS OF DEFECT SENSITIVITY STUDY}

Crack-tip stress intensity factors have been calculated for a set of representative defects of interest to reactor pressure vessel and piping integrity. Details of the study and results are described in Appendix $A$ to tio is report. Wall thicknesses of 4,8 and $12 \mathrm{in}$. were evaluated for stress levels from 20 to $60 \mathrm{ksi}$ both as membrane and bending stresses. Cracks of various 
geometric shapes, orientations and locations in the vessel wall were considered. The intent was to generate physically meaningful numbers for correlation with inspection and fracture toughness data.

The present defect sensitivity study was based entirely on linear fracture mechanics concepts. For materials of high fracture toughness it was recognized that elastic-plastic fracture mechanics would be required to give a more accurate picture of defect sensitivity. Nevertheless, the present results are believed to show correct trends as to the relative severity of different types of flaws.

A number of trends emerged from the calculations. From the standpoint of geometric effects, surface defects are somewhat more severe than internal defects and ID surface defects are siightly more severe than defects at the external surface. Also, for a given depth, elliptical type flaws are more severe than circular type flaws. The stress gradient effect is important mainly for small defects if the defect is located within the fieid of peak bending stresses. For large defects, high membrane stresses are of more concern than high loca? bending stresses. Further trends are described in Appendix A through a pictorial display of relative stress intensity factors.

\subsection{OVERVIEW OF CRITICAL FLAW SIZE ESTIMATES}

Critical flaw sizes have been estimated to establish required sensitivity ranges for NDE. The critical flaw size estimates in the present research program have been based largely on results of calculations performed at BattelleColumbus Laboratories (BCL). A draft report from the BCL (Mayfield et al. 1980) became available late in the final quarter of this study and evaluation of the results is continuing.

Scope of Calculations

In addition to the $B C L$ results, a series of independent calculations have been conducted at PNL to supplement the results of the $\mathrm{BCL}$ Cold Leg Integrity Evaluations. The calculations have been limited in scope, but provide an independent check on the $B C L$ work. Futhermore, these calculations provide a basis for evaluating the conservatism present in the BCL study, which was based on 
deterministic considerations and was intended to establish margins of safety against a large break in the cold leg piping system of a pressurized water reactor power plant. In contrast, the present research program is to provide results for eventual probabilistic evaluations and is directed to inspection rather than design requirements.

Both subcritical slaw growth and unstable fracture were considered. Two levels of inspection sensitivity were of interest. The first level was that needed to avoid piping system leaks from stable growth of Io surface flaws into through-wall flaws. The second level was to ensure that unstable crack growth toes not occur either for io surface or through wall flaws. Only subcritical crack growth by fatigue was considered. No attempt was made to estaibish critical slaw sizes on the bas is of intergranular crack growth by stress corrosion sracking, although envirommental effects on fatigue were evaluated.

The PNL calculations consisted of the following specisic flaw size evaludions which are documented below:

1. Critical flaw sizes were estimated basad on fatigue crack gruwth rates by extension of rasults generated by Combustion Engineering (1977). The sensitivity of predictions to variation in crack growtin rate curves (da/dn versus $\Delta k$ ) for reactor piping materials was emphasized. These calculations were to establish the size of flaw that cadid grow to bocome a through wall leaking flaw during a ten-year inspection interval.

2. Critical flaw sizes were estimated based on ASME Code allowable stresses, and the methodology of ASME Section XI. These calculations were intended to give a very conservative bower bound on the size of flow that could grow in an unstable manner.

3. Critical flaw sizes were estimated based on the dual criteria approacn (Chell 1979); limear elastic fracture mechanics and not section plastic collapse provided bounds on critical flaw sizes. These calculations considered the quession of flaw length as opposed to flaw depth. As such, througin wall flaws were consifered and flaw langthis likely to grow in an unstable manner were estimaced. 
4. Critical flaw sizes were estimated based on the recent work of Paris and associates using the concept of tearing instability (Paris et a). 1979a; Tada et a1. 1979; Paris et a1. 1979b). The focus was on very long and deep surface flaws which may become unstable before beconing letectable as leaking through-wall flaws. The main concern was the deep 360-degree circumferential flaw.

5. Piping flexibility calculations were performed to establish some bounds on dxial loads and moments for upset conditions. These calculations are reported in Appendix B.

To date, ID surface defects in only relatively large pipe sizes have been considered. In generat, smaller pipe sizes will present less demanding inspection requirements. As a $r$. critical defect sizes in a small pipe will represent a greater fraction of ". ¿pipe wall than the corresponding defect for a large pipe. Hence, flaws l. interest in sialler diameter piping should be more easily detected.

Conclusions of Flaw Size Estinates

The available analyses of the growth and stability of flaws in large size reactor piping have led to the following results and conclusions.

Failure Mode

1. Evaluations of the consequences of I0 surface flaws in piping indicates a high probability that flaws will prodice a leak before they will cause a pipe break. Therefore, the deficition of critical flaw sizes on the basis of unstable crack growth and fracture toughness considerations is of limited usefulness in establishing NDE sensitivity requirements for application to reactor piping systems.

2. The failure mode of primary concern should be an increase in crack growth rate such that an iD surface flaw breaks through the pipe wall witin a time span of the inspection interval (10 years).

3. In accordance with the leak-before-break condition, critical defect sizes for unstable crack growth have been found to be through wall flaws of significant length. For axial through-wall flaws, the 
jength is on the order of half a pipe diameter and for circumferential through wall flaws, the length is on the order of half the circumference of the pipe. For deep surface flaws, unstable crack growth probably requires very long lengths such as 360 degrees for circumferential flaws and several diameters for axial flaws. For such flaws, evaluations are tentative and require further advances and experimental verification of plastic fracture methods.

Variation in Critical Flaw Sizes

1. Evaluations of crack growth rates show significant differences in allowable defect sizes both from pipe to pipe and from location to location with a given pipe.

2. Even under the conservative assumptions of the BCL calculations ieg., levels of vibrational stress, fatigue crack growth rates and threshold ak levels), many welifs can tolerate quarter wall defects and achieve a 40-year design life.

3. Conservative andysis methods indicate a finite probability of failure at certain locations (particularly at dissimilar metal we?d5) within a time period of one percent or less of design life for flaws with depths as small as one tenth of the pipe wall.

4. Vevertheless, the overa!l trend of the resulis suggests a relatively low probability of failure by subcritical crack growth within a ten year inspection interval (say less than one percent) for flaws of depths less than 25 percent of tha pipe wall. ASME Code Evaluations

1. Application of linear elastic fractare mechanics following the procedures of Section XI of the ASME Code leads to critical flaw depths on the order of one-fourth of the pipe wall thickness. These predicted flaw sizes are roughly the same as those of fatigue crack growth calculations. This agreement, however, comes from a prescribed reduction of fracture toughness rather than consideration of the actual mechanisms ?.ading to plpe tal lure. 
Tearing Instability AnaIysis

1. Elastic plastic fracture mechanics has been applied to predict conditions for unstable crack growth through the concept of tearing instability. The main concern in the present study has been circumferential flaws that may grow in a relatively uniform manner through the wall of the pipe without breaking through and causing a detectable leak.

2. The tearing instability analyses indicate that unstable crack growth of circumferential flaws under displacement controlled bending loads (e.g., restraint of thermal expansion) requires pipe lengths much longer than relevant to reactor piping systems.

3. Under pure axial loadings of deep 360-degree circumferential flaws, unstable flaw growth is likely for flaw depths of about 80 percent of the pipe wall. Such growth can be caused by axial stresses solely from internal pressure or by displacement controlled axial loadings for pipe lengths as short as $20 \mathrm{ft}$. Severe axial loads (even of a displacement controlled source) could reduce the critical flaw depth to about 50 percent of the pipe wall. Further work is needed to establish whetiner there are credible sources of axial stress of the required type and magnitude to sustain such unstable fractures.

\subsection{CRITICAL FLAWS BASED ON FATIGUE}

Critical flaw sizes have been estimated based on fatigue crack growth rates by extension of results reported by Combustion Engineering (1977). The results were intended to supplement a much more extensive series of calculations performed by Battelle-Columbus Laboratories (BCL). The main purpose of the PNi study was to evaluate the conservatism present in the BCL study. In particular, tne BCL calculations atilized conservative crack growth curve from recent data published by Bamford (1979).

Combustion Engineering (1977) estinated fatigue crack growth rates for Combustion Engineering hot and cold leg piping designs for the loading transients in Table 4.1. The calculations were for 1.J-in.-deep inside surface 
flaws. As indicated in Figure 4.1, the baseline calculation reported for a 1.0-in.-deep flaw gave negligible crack growth for 100 years of operation. The Combustion Engineering results were based on the fatigue crack growth relationship

$$
d \lambda / J N=C(\Delta K)^{n} \text {, }
$$

where: $\quad \exists=$ crack depth, inch

$$
\Delta K=\text { stress intensity range, } k \text { si } \sqrt{i n} \text {, }
$$

with $C=3.97 \times 10^{-14}$ and $n=5.53$ taken to describe A516 Grade 70 ferritic step? at a $550^{\circ} \mathrm{F}$ operating temperature. If one assumes that to a first approximation sk is proportional to $\sqrt{a}$, then

$$
\frac{d a}{d N}=(\text { constant }) a^{2} \cdot 34
$$

By integration,

$$
v=c_{1}+c_{2} a^{-i .94}
$$

Enterpreting $N$ as the number of years of operation, the constants $C_{1}$ and $C_{2}$ can be selected to fit the Combustion Engineering results sinown in Figure 4.1 for an initial a: : l.0-in. flaw, for which

$$
\begin{aligned}
& N=0 \text { years, for } a=1.3 \text { in., } \\
& N=100 \text { years, for } a=1.113 \mathrm{in} .
\end{aligned}
$$

The rasidting general retationship is

$$
N=559\left(1-a^{-1.84} ;\right. \text {, }
$$

wich is plotted in Figure 4.1 for variods initial fiaw deptns. The resulting projections for smaller and larger initial flaws show that the inistal flaw mist be essentia? ly through the wall (thickness of 2.5 in.) before the predicted extent of crack aruwtin in the 70-year plant life is significant. 
IABLE 4.1. Reactor Coolant Systen Transients

\begin{tabular}{|c|c|}
\hline Transients & Frequency \\
\hline Heatup/cooldowr at $100^{\circ} \mathrm{F} / \mathrm{hr}$ & 12 per year \\
\hline $\begin{array}{l}\text { Loading/Un7oading } 0-100 \% \\
\text { 100\%-0 o } 5 \% / m i n\end{array}$ & 1 per day \\
\hline $10 \%$ full load steo & 1 per wazk \\
\hline Leak Test & 5 per year \\
\hline Turbine or reactor trips & 13 per year \\
\hline Hyidro Test & l every 4 years \\
\hline Loss of FTow & 1 per year \\
\hline
\end{tabular}

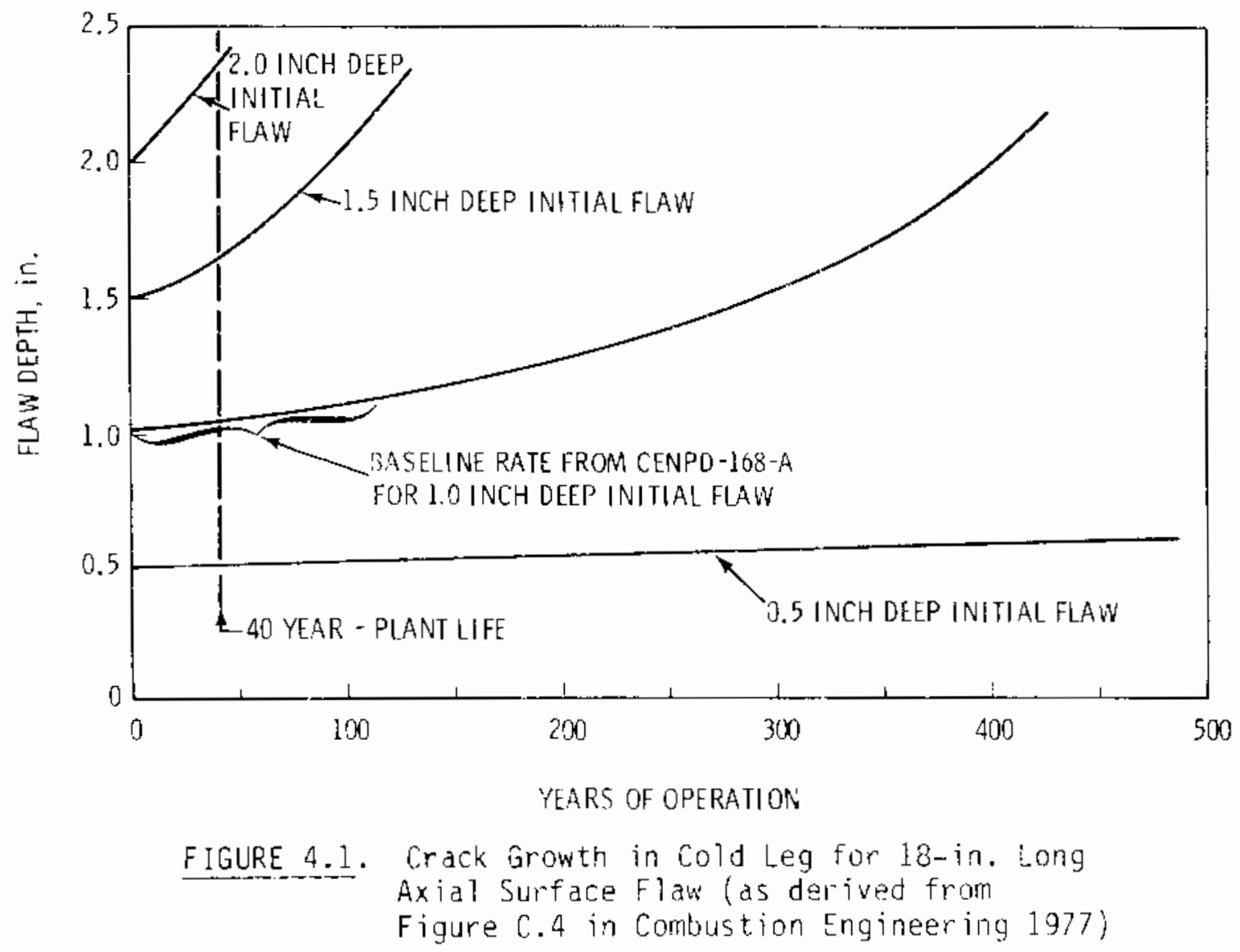


Figure 4.2 shows the fatigue crack growth curve used by Combustion Engineering (1977) compared with curves as recommended in ASME Section $x 1$ for a ferritic alloy. Aiso shown is the curve used in the BCL cold leg integrity evaluation (Mayfield et al. 1980). It was concluded that the Combustion Engineering results are unconservative for 10 surface flaws since the assumed crack growth rates were representative of an air environment rather than water reactor environment. A150 shown in Figure 4.2 are the results of applying factors of 2 and 4 on stress to the growth rate curve used in the Combustion Engineering calculation. A factor of 2.0 gives rates consistent with the ASME curve for the water enviroment. The curve for a factor of 4 can be viewed as a conservative estimate of crack growth rates which approximates the curve used in the $B C L$ cold leg eviluation.

Table 4.? gives critical flaw depth estimates based on calculated crack growth rates. Results of Combustion Engineering (1977) were adjusted to account for differing initial crack depths (using Equation 4-4) and different assumed fatigue crack growth curves. An initial flaw that could grow to a depth of one half the wall thickness during an inspection interval of 10 yeris was taken to be unacceptable. Flaw depth estimates are given for both the hot and cold legs of the Combustion Engineering design. Circumferential and axial flaws of two lengths were considered. 


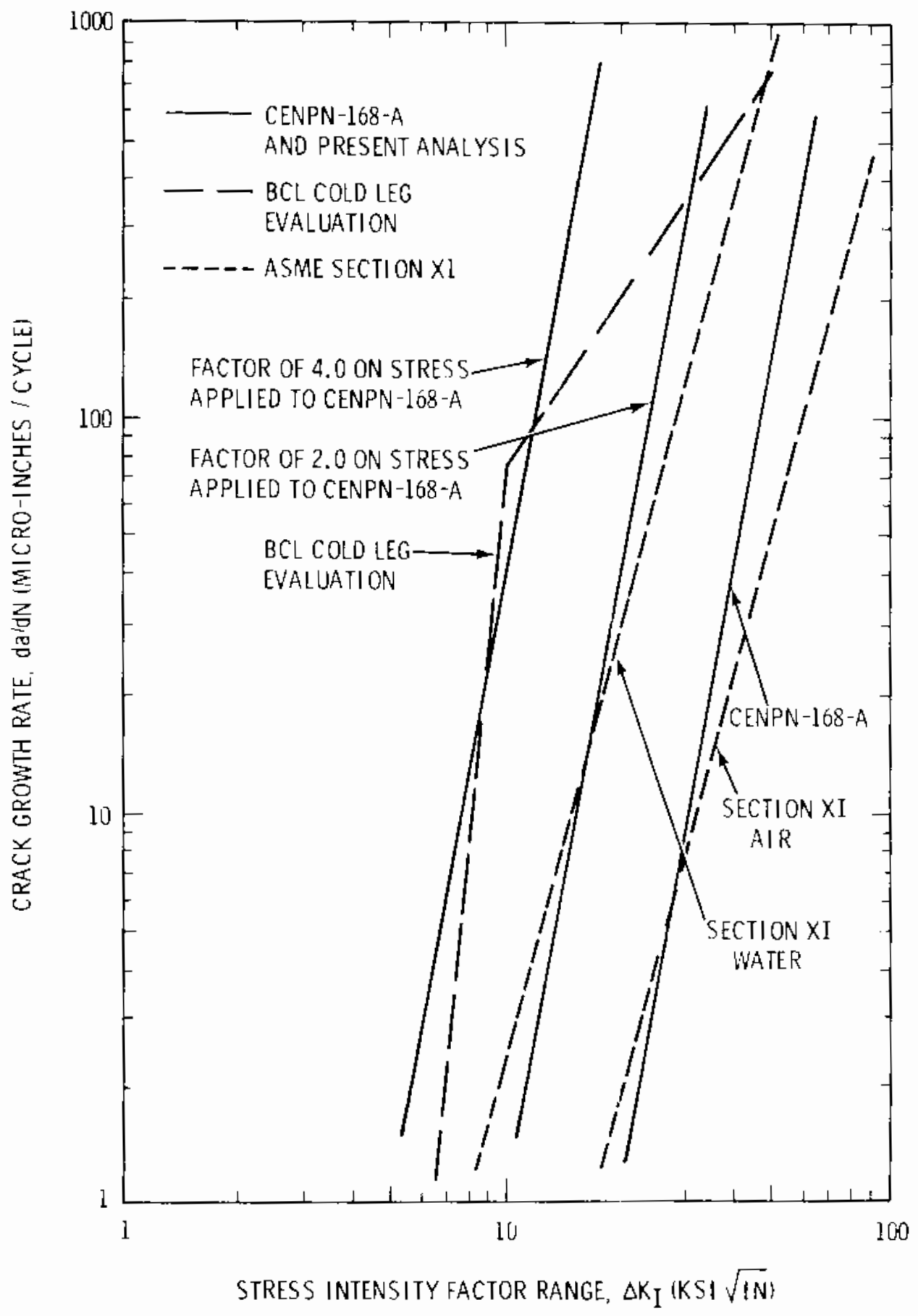

[IGURE 4.?. Fatique Crack Growt.1 Data for Ferritic Steels 
TABLE 4.2. Critical Flaw Size Estimates Based on

Fatigue Crack Growth

$\begin{gathered}\text { Allowable Flaw Depth, in. } \\ \text { Original } \\ \text { Length } \\ 8 \text { in. }\end{gathered}$
$\begin{gathered}\text { Original } \\ \text { Length }\end{gathered}$

Cold $\mathrm{Leg}$

$\begin{array}{llll}(30 \mathrm{in}[0 \times & \text { Circumferential Flaw } & 0.32 \text { to } 1.12 & 0.19 \text { to } 0.98 \\ 2.5 \text { in. Nall) Axial Flaw } & 0.19 \text { to } 0.96 & 0.12 \text { to } 0.78\end{array}$

Hot Leg

$\begin{array}{clll}\text { (42 in. ID } x & \text { Circumferential Flaw } & 0.15 \text { to } 1.02 & 0.12 \text { to } 0.91 \\ 3.75 \text { in. Wall) Axial Flaw } & 0.15 \text { to } 1.05 & 0.10 \text { to } 0.73\end{array}$

NOTES: Based on flaw growth to a depth of $t / 2$ at the end of a 10 -year inspestion interval.

Flaw growth rates estimated from curves in Figure $C .4$ of Combustion Engineering's (1977) report.

The range in flaw sizes indicates sensitivity of calculations to crack growth rate curve (da/dN vs $\Delta K$ ) used in the analysis.

Critica? flaw depths range from $0.10 \mathrm{in}$. to over $1.0 \mathrm{in}$, depending prilifarily on the degree of conservatism appliad in selecting the fatigue crack growti rate curve. The upper bound in flaw dept? corresponds roughly to the Section $X I$ water curve, whilo the lower bound corresponds roughly to the curve used in the BCL cold leg evaluation. A depth of about 0.50 in. might be viewed as a realistic estimate of the flaw depth which must be detected in NDE if through cracks are to be avoided.

The resilts presented here are intended to be indicative of requirements for ferritic piping materials. Oata as sunnarized by Marston (1973) indicate that srack growth rates for stainless steels are somewhat lower than those used here for ferritic materials. Thas, predicted critical f?aw depths adied on fatigue crack growth rates should be somewhat greater for stainless piping.

The results of Table 4.? can be compared with results of the $B C L$ colid leg integrity evaldation. Bot's studies suggest that fiaws of depth 0.25 in. or less can grow through the wall at locations of nigh stress in a time period of less tian a ten-year inspection interval. Such predicted growth rates, how- 
ever, correspond to the selection of conservative crack growth curves. Futherinore, the results here indicate that estimated critical flaw sizes are very sensitive to the particular crack growth rate data selected for the analysis. It is seen that range or uncertainty on crack growth rates can lead to a variation approaching 10:1 for predicted critical flaw depths.

\subsection{ASME SECTION XI FLAW SIZES}

Critical flaw sizes corresponding to unstable crack growth based on fracture toughness considerations were estimated lsing the methodology of Section $X I$ of the ASME Boiler and Prassure Vessel code. The intent of Section XI, as outlined by Marston (1978), is to ensure that the presence of a flaw does not reduce the factor of safety against burst below its nominal value of 3.0 . In this context the procedure of Section XI accepts flaws if the maximuln applied stress intensity is less than $k_{I C} / \sqrt{10}$.

Data compiled by Server and 0ldfield (1978) indicate that the appropriate value of $k_{\text {IC }}$ for ferritic piping snould be at least $250 \mathrm{ksi} \sqrt{i n}$. Tnus, applied stress intensity factors as calculated here by linear elastic fracture mechanics were required to he less than $K_{I C} / \sqrt{10}=79$ ksi $\sqrt{i n}$. For stainless piping the data indicate higher allowable stress intensity factors.

Critical flaw deptins were conservatively estimated by assuming that the piping system is stressed to the maximum level allowed by the ASME Code. For the Combustion Engineering cold leg (30-in. ID and 2.5-in. wall) of SA-516 Grade 70 at $550^{\circ} \mathrm{F}$, the design stress $S_{m}$ is $18.6 \mathrm{ks}$. Table 4.3 gives allowable flaw depths as calculated for internal surface flaws based on elastic stress intensity solutions reported by Becker (1379). Flaw depths are given for a $70 \mathrm{cal}$ membrane tension of $3 \mathrm{~S}_{\mathrm{m}}$ and a combined membrane and tension of $3 S_{\mathrm{m}}$.

As indicated, elliptical surface flaws (aspect ratio of 10) are more severe than surface half-penny flaws (aspect ratio of 2). Also, membrane tension is more severe than combined membrane and bending. The results of Table 4.3 show that detection of 0.50 -in.- deep flaws during inspection would be consistent with the requirements of ASME Section XI for even the worst combination of stress and flaw shape. It is probable that evaluations for more 
TABLE 4.3. Critical Flaw Size Estimates Based on ASME Section XI for Internal Surface Flaws

\section{Allowable Flaw Depth, in. Half Penny Elliptical}

$\begin{array}{lll}\text { Membrane Tension }=3 S_{\mathrm{m}} & 1.05 & 0.52 \\ \text { Combined Membrane a Bending } & 1.31 & 1.00 \\ \text { Membrane }=5 \mathrm{~m} & & \\ \text { Bending }=2 S_{\mathrm{m}} & & \end{array}$

NOTES: For Combustion Engineering cold leg witn $30 \mathrm{in.}$ ID and $2.5 \mathrm{in}$. wal1.

Fracture toughness of $250 \mathrm{ksi} \sqrt{\mathrm{in}}$. and Section XI allowable of $\mathrm{K}_{\mathrm{IC}} / \sqrt{10}=79 \mathrm{ksi} \sqrt{\mathrm{in}}$.

Design stress of $\mathrm{S}_{\mathrm{m}}=18.6 \mathrm{ksi}$

realistic stress levels would show that through wall cracks even for 2.5-in. wall piping would in many cases meet the stress intensity factor rimitations of Section XI.

\subsection{CRITICAL FLAWS BY DUAL CRITERIA APPROACH}

Critical flaw sizes are estimated here using the dual criteria approach (Chell 1979). The approach in these calculations is less conservative than that of ASME Section XI as described above in that fracture toughness values used in the calculations are not derated by the factor of $1 / \sqrt{10}$. In this sense actual flaw sizes for catastrophic failure are estimated rather than the flaw size which will reduce the factor of safety against burst below the desired 3.0 value of the ASME code.

A common fracture toughness value $K_{I C}=250 \mathrm{ksi} \sqrt{\mathrm{in}}$. was used in all evaluations by linear elastic fracture mechanics (LEFM) for both ferritic and stainless piping. This value should be conservative for all the materials considered. However, this use of a common value for all materials, probably fails to show trends due to higher toughness levels of stainless materials relative to ferritic materials. 
Interpretation of fracture data in terms of the failure analys is diagram approach suggests that critical flaws can bl evaluated using a dual criteria approach chell (1979). The dual criteria approach requires an evaluation in terns of linear slastic fracture mechanics combined with an evaluation of the plastic collapse or limit load for the cracked structure. In the following, critical flaw sizes are estimated for large-iameter piping with flaws of tne through-wall type. Part-through flaws can be shown not to be critical, mless they are both very deep and have a very large aspect ratio. The stability of such flaws is considered later using the tearing instability concept.

Predicted lengths for through flaws for cola leg piping of various reactor designs are summarized in Table 4.4. Design parameters werta taken from Eiber at al. (1979). The discussion below describes the equations and methods used to predict the critical flaw paraneters. Predictions were made first for failure due to pressure-induced stresses alone, then for these pressure stresses combined with therinal, residual stresses, and piping flexibility stresses. The approxinate method used to account for thermal and rasidial stresses is described in Appendix B, along with estinates of piping fiexibility stresses.

Axial Flaw $=$ LEFM

Newman (1975) reviews solutions for axial through cracks in cylindrical pressure vessels and presents the folias (1965) solution in the following form:

$$
k_{1}=S_{n} \sqrt{\pi C} F\left(\lambda_{t}\right), I \leq \lambda_{t} \leq 10
$$

where

$$
\begin{aligned}
2 c & =c r a c k \text { length } \\
S_{n} & =p R / t \\
p & =\text { pressure } \\
R, t & =\text { radius and wall thickness } \\
\lambda_{t} & =c / \sqrt{R t} \\
F\left(\lambda_{t}\right) & =\left(1+0.52 \lambda_{t}+1.27 \lambda_{t}^{2}-0.074 \lambda_{t}^{3}\right)^{1 / 2} .
\end{aligned}
$$


The furiction $F\left(\lambda_{t}\right)$ accounts for the effects of shell curvature on stress intensity factor.

Numerical evaluations for critical lengths of axial flaws in cold leg piping are indicated as items 7 and 8 in Table 4.4. For pressure laading only (neglecting therinal and residual stresses), the predicted critical lengths are on the order of $15 \mathrm{in}$. Consideration of thermal and residual stresses by the method of Appendis B reduces the critical flaw lengths by only about? in. It should be noted that a conservative value of $\mathrm{K}_{\mathrm{IC}}=250 \mathrm{ksi} \sqrt{\mathrm{i}}$. has been used in the calculations, whereas some data suggest $k_{\text {IC }}$ values as much as two times greater than this value may be appropriate. Use of such higher values for $k_{I}$, would result in a four-fold increase in predicted critical flaw length (i.e., from 15 to 60 in.).

Axial rlaw - Net Section Collapse

While theoretical solutions for net section plastic collapse for axial flaws in cylinders have been given by Erdogan et a1. (1975), predictions here are based on empirical correlations. Such correlations of pipe tests are given for example by Esselman et a1. (1976) and are based on data from BattelleColumbus Laboratories (Keicner et al. 1973) and General Electric (Reynolds 1968). Results are presented as the ratio of failure stress $\mathrm{J}=p(R / t)$ to flow stress as a function of the parameter $\lambda=c / \sqrt{R t}$. Burst test results froil defacted stean generator tubes at PNL (Alzheimer et al. 1979) have also been applied and the predictions generally agree witn data from larger pipe. An empirical equation fit to the PNi results is given by Alzheimer et al. as

$$
\sigma / \sigma_{0}=(1-a / t)+a / t 2-0.746(c / \sqrt{R t})
$$

where a is the flaw depth with $\mathrm{a} / \mathrm{t}=1.0$ corresponding to a through-wall $\mathrm{flaw}$. Predicted critical lengths for net section callapse of axial through-wall flaws are listed as item 9 in Table 4.4. Dredictions of net section col.lapse for axial flaws should not be governed by thermal, residilal or piping 
TABLE 4.4. Critica] Lengths of Through-wall FTaws in cold Leg as Predicted by Dual Criteria Approach

\begin{tabular}{|c|c|c|c|c|c|c|c|}
\hline & & & & \multicolumn{2}{|c|}{$5 \sin$} & \multicolumn{2}{|c|}{ westirighouse } \\
\hline & & & $\begin{array}{l}5 \overline{4516} \\
6 \mathrm{r} 70 \\
\end{array}$ & $\begin{array}{l}5 A 105 \\
\text { Gr C }\end{array}$ & $\begin{array}{l}5 A 516 \\
\text { Gr } 70 \\
\end{array}$ & $\begin{array}{l}5 A 376 \\
6 r 304\end{array}$ & $\begin{array}{l}S A 351 \\
\text { Gr CFBA }\end{array}$ \\
\hline DESIGN PARAMETERS & 1) & Yicld Strength, psi & 29,400 & 34,100 & $? 9,400$ & 18,800 & 20,700 \\
\hline \multirow{4}{*}{ ANO MATERIAL STRENGINS } & 2) & Uitimate Strength, psi & 53,800 & 68,250 & 55,800 & 50,400 & 48,000 \\
\hline & 3) & Flow Stress, psi & $4 \%, 600$ & 51,175 & 42,600 & 34,600 & 34,350 \\
\hline & 4) & wall Thickness, $t$, in. & 2.15 & $7.06,25$ & 2.0625 & 2.38 & $? .32$ \\
\hline & $5)$ & Radius, $R$, in. & $16 . ? 5$ & 13.03 & 15.03 & 14.91 & 14.91 \\
\hline RESUI TS FOR AXIAL. & 6) & $\because=p(R / t), p S:$ & $: 4,300$ & $15,03 \%$ & $i 6,032$ & 14,138 & 14,138 \\
\hline \multirow[t]{3}{*}{ FLAWS } & 7) & 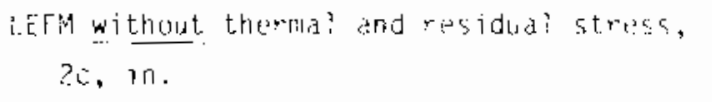 & 16.4 & 13.9 & 23.7 & 15,2 & $i 5.5$ \\
\hline & 8) & $\begin{array}{l}\text { LiFM with therinal anc residual stress, } \\
2 e \text {, in. }\end{array}$ & $14 . \%$ & 11.8 & $i 1.3$ & $24 . i$ & 33.9 \\
\hline & ij & $\begin{array}{l}\text { Net section collapse, with and without. } \\
\text { therinal and residuai streus, ?c, ir. }\end{array}$ & 11.9 & 71.7 & 10.7 & $i \dot{i} \cdot{ }^{\prime}$ & $i f . j$ \\
\hline \multirow{5}{*}{ RESULTS FOR CORCUMFFREN- } & 10; & $0=p(R / P t), D S i$ & $?, 161$, & $\therefore .96$ & $4, \therefore 1$ & 7,060 & , \\
\hline & 11 & 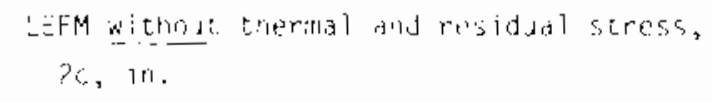 & $\begin{array}{r}0.3 .3 \\
10\end{array}$ & $\begin{array}{r}44.7 \\
: 16 y^{2}:\end{array}$ & $\begin{array}{l}\operatorname{sy.} 7 \\
\left(199^{6}\right)\end{array}$ & $\begin{array}{r}37.3 \\
\left(200^{0}\right)\end{array}$ & $\begin{array}{l}37.3 \\
\left.2014^{0}\right)\end{array}$ \\
\hline & $\mathrm{i} \% \mathrm{j}$ & $\begin{array}{l}\text { iffM with lherind and residual stress, } \\
\text { zc, in. }\end{array}$ & $\begin{array}{l}: 9 .: \\
: 69^{2}:\end{array}$ & - & -- & $-\cdot$ & -- \\
\hline & $13)$ & $\begin{array}{l}\text { Net section: co: apse without thermit anc } \\
\text { residuai stress, ro, in. }\end{array}$ & $\begin{aligned} 5: 3 \\
1.32\end{aligned}$ & $\begin{array}{r}48.8 \\
\left(180^{2}\right)\end{array}$ & $\begin{array}{r}45.6 \\
\left(174^{\circ}\right)\end{array}$ & $\begin{array}{r}43.7 \\
\left(150^{\circ}\right.\end{array}$ & $\begin{array}{r}43.7 \\
103^{\circ}\end{array}$ \\
\hline & 14) & 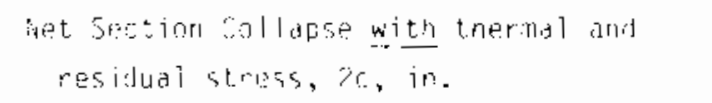 & $\begin{array}{r}30.4 \\
i 167^{0} !\end{array}$ & - & -- & $\cdots-$ & -- \\
\hline
\end{tabular}


flexibility stresses and thus only results for pressure stresses are given. The values given were based on the design curve of Esselman et a). (1976); evaluations based on the empirical Equation (4-6) gave predicted flaw lengths which in certain cases were somewhat shorter.

The predicted critical flaw lengths were on the order of 16 to 22 in., which corresponded to about $1 / 2$ to $3 / 4$ of the pipe diameter. These predicted lengths were slightly longer than those predicted on the bas is of linear elastic fracture mechanics.

Circumferential Flaws - UEFM

The solution used for evaluation of elastic stress intensity factors for circumferential through flaws was one due to Gilinan (1968) as reported by Eiber et al. (1979). The solution has the form

$$
K_{I}=\pi c\left[\frac{p R}{2 T} f_{i}(x)+\frac{i}{R^{2} t} f_{2}(B)\right]
$$

where

$$
\begin{aligned}
2 \mathrm{C} & =\text { crack length } \\
2 \alpha & =\text { crack angle }=c / a, \text { radians } \\
M & =\text { bending moment on pipe cross section. }
\end{aligned}
$$

The functions $f_{1}$ and $f_{2}$, given in graphical form by $E$ iber et al., are equal to 1.0 for small crack angles and attain values on the order of three for a 180-adegree flaw.

Predicted lengths of critical flaws neglecting residua?, themal and piping flexibility stress are listed as item (11) in Table 4.4. Predictedf faw lengtis are on the order of 50 to $60 \mathrm{in}$. and extend around more than onehalf of the pipe circumference.

Residual and therend stresses along with bending moments due to piping flexibility were accounted for using the results of Appendix $B$. Predicted critical flaw lengths were reduced by a factor of about three. A sinall part 
of this change came from a reduction in fracture toughness to account for residual stresses and those therma? stresses due to through-wall temperature yradients. The major factor, however, was the additional stress due to a benoing moment included to account for thermal expansion effezts in the piping system. This amounted to an axial bending stress of 17,500 psi supprimposed on tne uni-. form 7000 to 8000 psi pressure induced axial stres; (pR/2t). Preaicted lengths of critical ajrcunferential flaws (item 12 of Tabla 4.4! iolucje the offect of thermal and residual stresses.

Circunferential claws - Net Section coll apse

The axial load and bending mollent ti) cause fully plastic deformation of a circumferentially cracked pipe can be readily cal:alated by considering strosses in the remaining 1 . ment of tine cracked section. Kanninen et al. (1975) have analyzed circiunf alially cracked pipas for through-wall cracks and related the moment and dxial loas capacities of tie pipe to the length of the crack. These results can be expressed as

$$
\begin{aligned}
& M_{b}=2 \sigma_{0} R^{2} t(2 \sin \beta-\sin x) \\
& B=\pi / 2(1-Q)-x / 2 \\
& Q=\frac{1}{\sigma_{0}} p R / 2 t+\frac{p}{d x i a r}-\frac{A}{A}
\end{aligned}
$$

where

$$
\begin{aligned}
& \sigma_{0}=\text { flow stress } \\
& 2 \alpha=\text { crack angle } \\
& \beta=\text { angle locating neutral axis in bending } \\
& P_{\text {axial }}=\text { axial load exclusive of axial load inposed by internal pressure }
\end{aligned}
$$

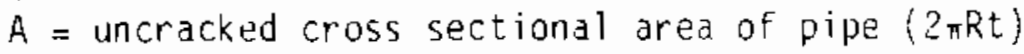

$$
\begin{aligned}
& M_{b}=\text { bending moment on pipe cross section }
\end{aligned}
$$

This set of equations can be solved by trial and error for the crack angle $2 \alpha$ corresponding to a given set of loadings $\left(p, P\right.$ axial, and $\left.M_{b}\right)$. It should 
be noted that the analysis includes the bending moment on the net section from the pressure induced axial load $(p R / 2 t)$ which is eccentric relative to the neutral axis in bending for the cracked section.

Table 4.4 lists under item 13 calculated critical flaw lengths and angles for net section collapse for the case where only internal pressure bading is present. Flaw lengths are in the 40- to 50-in. range. These lengths correspond to through flaws extending over about one-half the circumference.

The critical flaw lengths are reduced by external bending noments due to therinal expansion effacts. Table 4.4 lists as item 14 the calculated critical flaw lengths for a bending moment in the pipe sufficient to give an axial bending stress of 17,500 psi. Imposition of this bending moment results in reduction in critical flaw length by about 40 percent, with the critical flaw extending around approximately $1 / 4$ of the pipe circumference.

\subsection{TEARING INSTABILITY ANALYSES}

In this section, the possibility of unstable crack growth by ductile tearing is eviluated using the concept of tearing instability. Both axial and circumferential flaws are considered. The main concern is witn deep surface flaws. In particular, the possibility that unstable growth of these flaws may occur before they grow by fatigue in a stable manner to become a leaking through-wall flaw is evaluated.

Recent fracture mechanics studies by $\mathrm{Par}$ is and $\mathrm{h}$ is associates have developed the concept of tearing instability (Paris et al. 1979a, 1979b; Tada et al. 1979). The method of analys is is applicabie to high-toughness materials, and states that three criteria for unstable crack propagation by ductile tearing mast be met as follows:

1) The flawed structure is in a state of net section yield.

2) The crack tip deformation must be sufficient to satisfy the elasticplastic toughness parameter $J_{I C}$ for the initiation of crack extension.

3) The slope of the $J$ versus crack extension curve of the material (as characterized by the Paris material tearing modulus) must be less than the increase of the applied value of $J$ with respect to crack length. 
Analyses of a number of simple structural configurations have been reported in the literature, along with conditions for unstable crack growth by ductile tearing. A significant trend of these results is that the above criteria do not predict a critical crack size (depth and length), per se, but rather dictates minimum structural dimensions (e.g., pipe length) needed for unstable tearing.

In the discussion that follows, three solutions that are relevant to piping flaws are presented and evaluated. In addition, data for plastic fracture properties (JIC and tearing modulus) for piping materials are reviewed.

Plastic Fracture Properties

The material parameters of interest are the flow stress, $\sigma_{0}$, the plastic toughness parameter, $\mathrm{J}_{\mathrm{IC}}$, and the tearing modulus, $\mathrm{T}_{\text {mat, }}$, which is a measure of the slope of the crack growth resistance curve (Japplied versus $\Delta$ a). Piping materials are classifjed here broadiy as either ferritic steels or stainless steels, and typical properties for each have been reviewed. Table 4.5 summarizes the elastic-plastic fracture parameters which were used in flaw evaliations. The temperatures of interest were in the 500 to $600^{\circ} \mathrm{F}$ $r$ ange.

Bamford and Bush (1979) present applicaule data for stainless steel castings, forgings and plate at $600^{\circ} \mathrm{F}$. Their data indicate tearing modulus va - $_{-}$ wes ranging from 225 to 612 (dimensionless) depending in large measure on test method. By definition, tearing modulus is

$$
T_{\text {mat }}=\frac{E}{\sigma_{0}^{2}} \frac{d J}{d a}
$$

where the flow stress and elastic modulds for stainless steels are taken as $34,350 \mathrm{psi}$ and $25 \times 10^{6}$ psi, respectively. The Barnford and Bush data also indicate $J_{I C}$ values ranging from 1500 to 2737 in.-lb/in.?. For analyses reported below, values of $J_{I C}$ and $T_{\text {mat }}$ for stainless steel piping have been conservatively taken to be $1500 \mathrm{in.}-1 \mathrm{~b} / \mathrm{in}$. ? and 200 , respectively. 
TABLE 4.5. Plastic Fracture Properties Used in Flaw Evaluations $\left(550^{\circ} \mathrm{F}\right)$

$\begin{array}{lcc} & \text { Ferritic } & \text { Stainless } \\ \text { Flow Stress, psi } & 42,600 & 34,600 \\ \text { Elastic Modulus, psi } & 28 \times 10^{6} & 25 \times 10^{6} \\ J_{\text {IC, in.-ib/in.? }} & 500 & 1,500 \\ T_{\text {mat }} & 100 & 200 \\ J_{\text {max }} \text { in.-10/in? } & & \\ \text { (for a } \cong 0.50 \text { in. of extension) } & <10,000 & <20,000\end{array}$

Data for the ferritic steels at temperatures near $550^{\circ} \mathrm{F}$ are lacking. wilson (1979) reports results at room temperature for A516 which shows J IC values in the range 570 to $855 \mathrm{in-1b} / \mathrm{in}^{2}$, and a tearing modulus of about 160 . Andrews and Shih (1979) and Server (1979) report data for A533 ferritic steel at 200 and $350^{\circ} \mathrm{F}$. At $200^{\circ} \mathrm{F} \mathrm{J}_{\mathrm{IC}}$ was in the range of 1500 to $2500 \mathrm{in.-10/}$ $\mathrm{in}^{2}$, and $\mathrm{T}_{\text {mat }}$ was in the range of 125 to 250 . At $350^{\circ} \mathrm{F}$, J IC was in the range of 650 to $1900 \mathrm{in-1b} / \mathrm{in}^{2}$, and $\mathrm{T}$ was in the range of 100 to 170 . This data indicates a decrease in the toughness parameters with increasing temperature. In the analyses below $J_{I S}$ and $T_{\text {mat }}$ for ferritic steel piping have been taken to have the values $500 \mathrm{in}-1 \mathrm{~b} / \mathrm{in}^{2}$ and 100 , respectively. Deep Axial Surface Flaw

Paris et al. (1979a) have derived the conditions for a tearing instabiiity for a deep axial surface flaw of depth = a, and length $=2 c$ in a wall section of thickness $=t$. The loading was internal pressure. The remaining ligament was taken to be yielded, but the nominal stress in the uncracked structure was below yield. A deep flaw in this context was one for which $\mathrm{d} / \mathrm{t}$ $\geq 1 / 2$.

The general criterion for tearing instability lead to the condition

$$
\frac{4 c}{t} \geq T_{m a t}
$$


for unstable extension of the flaw through the wall. It should be noted that the stability is not dependent on flaw deptin but rather on the length of the flaw. The predicted critical length of a deep surface flaw that can experience a ductile tearing instability is then

$$
2 c=(t / 2) T_{\text {mat }}
$$

Using the tearing modulus valies of Table 4.5 and a nominal wall thickness of 2.5 inch for the cold leg, critical flaw lengths for unstable growth of the deep surface flaw through the wall are:

$$
2 c= \begin{cases}125 \text { in. (4 diameters), } & \text { ferritic stee } \\ 250 \text { in. }(8 \text { diameters }), & \text { stainless steel }\end{cases}
$$

For deep axial surface flaws these results predict that flaws will not break through the wall in an unstable manner unless the flaws are quite long relative to the pipe dianeter. It is thus implied that deep surface cracks will tend to break througin and become leaking through-wall cracks only by growing stably by a mechanism such as fatigue.

Circumferentia! Flaw in Bending

Tada et al. (1979) have considered tearing instabilities in piping under displacement controlled bending loads, for the case of circumferential flaws. Details of the analysis are not discussed here. It is shown in Tada et al. that:

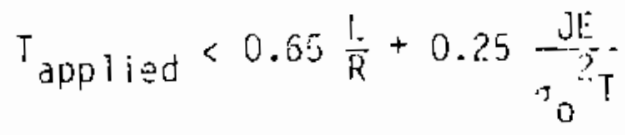

where 


$$
\begin{aligned}
L & =\text { lengtin of pipe } \\
R & =\text { mean radius of pipe } \\
J & =\text { value of } J \text { integral for applied bending loading } \\
\sigma_{0} & =\text { flow stress } \\
E & =\text { elastic modu?us }
\end{aligned}
$$

The constants in this inequality were selected to bound Tapplied for all circumferential flaws, both of a purely surface type and through-wall type, as well as surface flaws which have broken through only a portion of a pipe circuinference.

The tearing modulus analysis predicts a minimum length of pipe required for unstable propagation of a circumferential crack for the applied displacement controlled bending loads. Using the fracture properties of Table 4.5 , the critical pipe length for ferritic and stainless steel for various pipe dianeters is given in Table 4.5 .

Table 4.5 indicates that unstable ductile fracture can occur in pipes of shorter length as the pipe diameter becones smaller. For the larger diameters, very long lengths of pipe are required to supply the stored elastic energy at a sufficient rate to support ductile fracture. Since the required piping lengths exceed those used in reactor systems, such piping systems have been termed "fracture proof" in Tada at al. (1979).

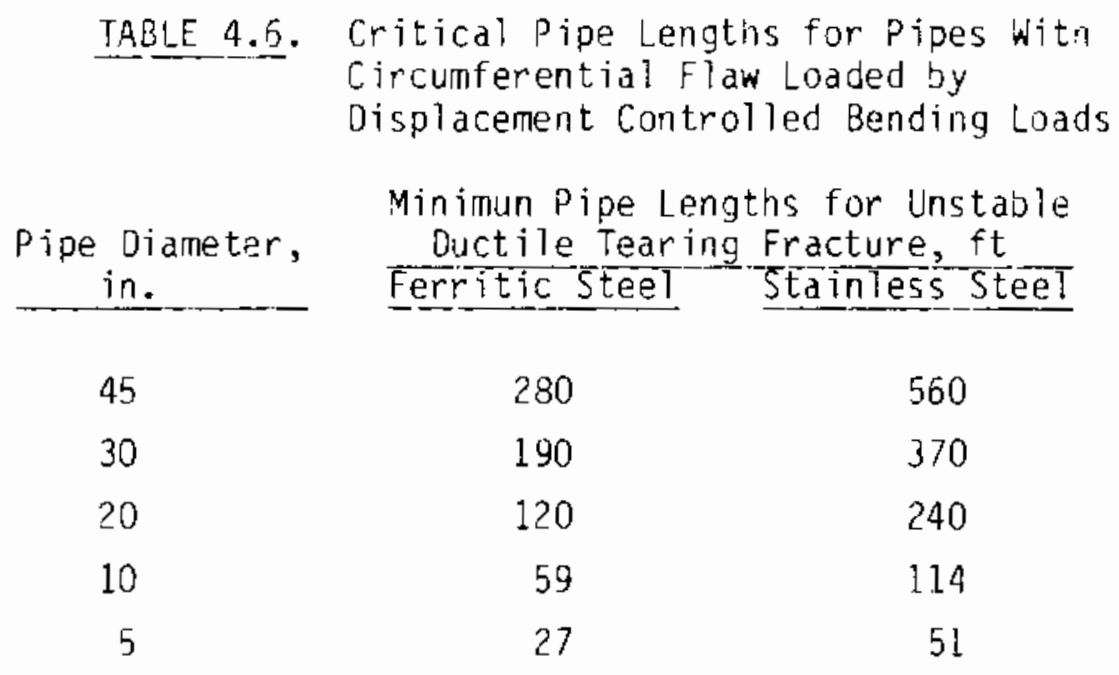




\section{Circumferential Flaw in Tension}

The conditions for a tearing instability in a straight length of pipe in tension are derived below following the methods of Paris (Tada et al, 1979). A pipe is given an imposed axial displacement, such as due to restraint of free thermal expansion, which places the pipe in a state of tension. A partthrough 360-degree circumferential fiaw of uniform depth around the circumference is assumed to be present in the pipe. The following definitions are made:

$$
\begin{aligned}
& =\text { length of pipe } \\
t & =\text { wall thickness } \\
a & =f l a w \text { depth } \\
P_{L} & =\text { limit load for flawed cross section } \\
R & =\text { radius of pipe } \\
\sigma_{0} & =\text { flow stress } \\
E & =\text { elastic modulus } \\
A & =2 \pi R a=\text { area of flaw } \\
\Delta & =\text { imposed axial displacement. }
\end{aligned}
$$

It is implied that the net section of the flawed pipe is yielded so tnat the net stress equals the material flow stress. The mechanical work to deform the pipe is as follows:

$$
U=U_{\text {elastic }}+U_{\text {plastic }}
$$

where

$$
\begin{aligned}
& U_{\text {Qlastic }}=\pi s_{0}^{2} R(t-a)^{?}(L / E t) \\
& U_{\text {plastic }}=2 \pi \sigma_{0} R(t-a)\left[\Delta-(t-a) \sigma_{0}(L / E t)\right]
\end{aligned}
$$


so that

$$
U=2 \pi R \sigma_{0}(t-a) \Delta-\pi R \sigma_{0}^{2}(t-a)^{2}(L / E t)
$$

The applied J value is

$$
J=d u / d A=-(d(J / d a)(d a / J A)
$$

Differentiating and combining terms gives the following result for the applial J value

$$
u_{\text {applied }}=\sigma_{0}[\Delta-(\partial, L / E)(l-a / t)] .
$$

By definition, the applied value of the tearing paraneter $T$ is:

$$
T_{\text {applied }}=\left(E / \sigma_{0}^{2}\right)(d J / d a)
$$

which in tis is case is simply

$$
T_{\text {app lied }}=L / t
$$

The three conditions for unstable ductile crack growth or tearing that must be satisfied simultaneously are:

(1) applied net stress $\geq$ flow stress

(2) crack initiation, Japplied $\geq]_{\text {I }}$

(3) tearing instability, Tapplied $\geq T_{\text {material }}$

These three criteria have been evaluated for the following range of parameters of interest to reactor piping: 


$$
\begin{aligned}
J_{\text {IC }}= & 500,1000,2000 \text { in-1D } / \text { in }^{2} \\
T_{\text {mat }}= & 50,100,200 \\
\sigma_{0}= & 35,000,50,000 \text { ps } i \\
\sigma_{a}= & \text { applied axial stress to unflawed pipe section } \\
& (10,000,20,000,30,000 \text { psi }) \\
E= & 26 \times 10^{6} \text { psi } \\
L= & 10,20,50,100 \text { feet } \\
t= & 0.5,1.0,2.54 .0 \text { inch }
\end{aligned}
$$

Under criterion (1) the net section of the pipe must experience net section yield so that

$$
a / t \geq\left(1-\sigma_{a} / \sigma_{0}\right)
$$

Table 4.7 lists ninimum flaw depths for net section yield for a range of axial stesses.

For evaluation of the crack initiation criteriun (2) one may express the fixed elongation of the pipe as

$$
\Delta=z_{\mathrm{a}} \mathrm{L} / \mathrm{E}
$$

and then express the crack initiation criterion as

$$
a / t \geq E J_{1 C} / \sigma_{0} ? L+\left(1-\sigma_{a} / J_{0}\right)
$$

This gives tile minimum crack depth to initiate the ductile fracture process. It is seen that the allowable flaw depth decreases as the pipe length and applied stress increases. Table 7.7 gives minimum crack depths. Pipes of lengths characteristic of reactor designs with a displacenent controlled stress of $10 \mathrm{ksi}$ could see the initiation of stable ductile fractura for surface flaws of about $75 \%$ of the wall. However, for unstable extension of such flaws the tearing instability criteria must also be satisfied. 
For tearing instability as defined by criterion (3) to occur, the pipe lengtin must be sufficient to satisfy the equation $L / t \geq T_{\text {material }}$. Table 4.7 indicates that unstable fracture can occur for thinner wall pipes as short as two feet, provided that the flow is of sufficient depth and axial piping loads are sufficiently great.

For a ductile tearing instability to occur, all three of the criteria as evaluated in Table 4.7 must be satisfied. The critical flaw deptins of Table 4.8 are intended to give representative predictions for a typical $20-f t$ length of reactor piping with a deep circumferential flaw under axial tensile loading. Results for both ferritic steels ( $J_{I C} \geq 500$ in.-lb/in.? and $T_{\text {mat }} \geq 100$ ) and stainiess steels $\left(\mathrm{J}_{\mathrm{I}} \geq 1500 \mathrm{in.}-\mathrm{ib} / \mathrm{in}\right.$ ? $^{\text {? }}$ and $\mathrm{T}_{\text {mat }} \geq 200$ ) are indicated. The range in wall thickness $(0.5$ to $4.0 \mathrm{in.})$ is intended to span pipe diameters from 6 to $30 \mathrm{in.}$ and greater. No attempt was made to identify the two applied axial stress levels of 10,000 and 20,000 psi with particular loading sources. The levels could be considered bounds on the combined effects of pressure, restraint of thermal expansion, seismic loading, water shugging, etc.

The results of Table 4.9 indicate that smaller pipe sizes may have a greater probability of unstable ductile fracture, since critical pipe lengths are shorter for these sizes. The results labeled "no instability" in Table 4.3 are those for which the assumed $20-\mathrm{ft}$ pipe length is insufficient to support a predicted tearing instability. The critical flaw depths range from 54 to $85 \%$ of the wa11. These depths are for a displacement controlled situation where both ends of the pipe are fixed so that the total axial extension of the pipe does not change as the crack grows.

The other limiting condition is one of a pipe under purely pressure loading where the ends of the pipe can move freely as the crack grows. It is significant to note that the critical flaw depths for such pure pressure induced failure are on the order of $80 \%$ of the wall. A full circumferential flaw of this depth will reduce the net pipe cross section to the point that the net section stress will exceed the ultimate strength level of the pipe. 
TABLE_4.7. Critical Flaw Depths and Critical Pipe Lengths for Unstable Tearing of Deep 360-Degree Circumferential Flaw in Pipe Loaded in Axial Tension

CRITERIA (1) - NET SECTION YIEID

cnet $\geq c_{0}$

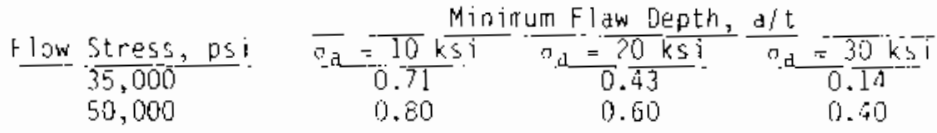

CRITERIA (?) - CRACK INITIR:ION

Japplied $\geq \mathrm{J}[\mathrm{C}$

$\left\{\mathrm{o}_{0}=35,000 \mathrm{psi}\right)$

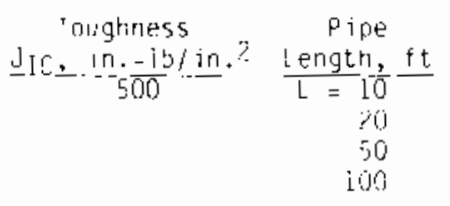

1000

$$
\text { i. }-10
$$$$
30
$$$$
50
$$$$
100
$$

2000

$$
\begin{aligned}
& L=10 \\
& \text { ำ } \\
& 130
\end{aligned}
$$

CRITERIA :2I - CRACK INITIA :OA

$$
\begin{aligned}
& J_{a p p} 1 \text { ied } \geq j l i \\
& \left.a_{0}=50,000 \text { psi }\right)
\end{aligned}
$$

\begin{tabular}{|c|c|c|c|c|}
\hline learing & & ONical in & engths, + t & \\
\hline Mod:uliss & $\because-0.5 \mathrm{in}$ & $t=20$ & $t=2.5 i n$ & $t=4.0-i n$. \\
\hline $\begin{array}{r}50 \\
100\end{array}$ & $\begin{array}{l}7.1 \\
4.1\end{array}$ & $\begin{array}{l}1 . ? \\
3.3\end{array}$ & $\begin{array}{l}10.4 \\
20.8\end{array}$ & $\begin{array}{l}16.7 \\
33.3\end{array}$ \\
\hline 200 & 3.6 & 15.7 & 4.27 & 66.1 \\
\hline
\end{tabular}

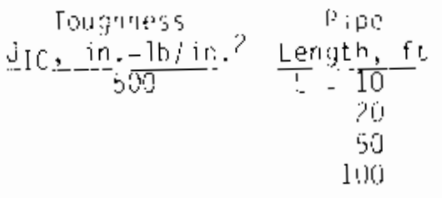

$$
\begin{aligned}
& \text { lioio } \\
& L=\begin{array}{r}
50 \\
03 \\
50 \\
100
\end{array} \\
& 20179 \\
& \begin{array}{r}
\square-10 \\
\quad 0 \\
50 \\
100
\end{array}
\end{aligned}
$$

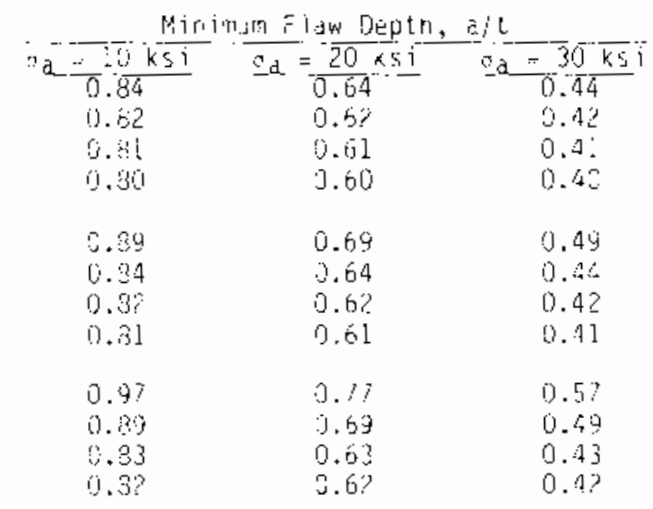

CRITERIA (3) - TEARINEINSTASIL: TV

Tappiied ? Traterial 
TABLE 4.8. Critical Flaw Depths for 20 Foot Length Pipe by Ductile Tearing for Deep 360 Degree Circumferential Flaw loaded in Axial Tension

FERRIIIC STEEL: $J_{I C}=500$ in.-1b/in.? and Tmaterial $:=100$

Wali Thickness,

$-\frac{i n .}{0.5}$

1.0

2.5

4.0
Flaw Depth, a/t

$\begin{array}{cr}\sigma_{a}=10,000 \text { psi } & 0.55 \\ 0.78 & 0.55 \\ 0.78 & 0.55 \\ 0.78 & =20,000\end{array}$

1.0 (no instability) 1.0 (no instability)

STAINLESS STEEL: JIC $=1500 \mathrm{in} .-\mathrm{b} / \mathrm{in} .2$ and T $_{\text {materia }}=200$

Wall Thickness, Flaw Depth, a/t

in.

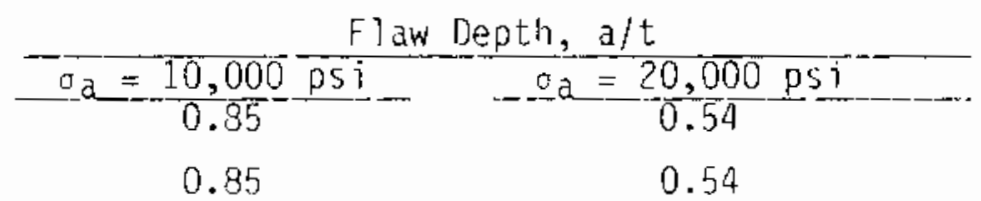

1.0

1.0 (no instability) 1.0 (no instability)

4.0

1.0 (no instability) 1.0 (no instability) 



\subsection{STATISTICAL DESIGN AND ANALYSIS}

This discussion details the statistical methods and procedures used to evaluate the effectiveness of in-service inspection of primary system piping. A primary objective of this program is to determine the effectiveness of inservice ultrasonic inspection. The objectives of statistical analysis here are to guide experimental design, maximize cost effectiveness of measurement programs, and provide the analysis necessary for evaluating inspection effectiveness. Section 5.1 defines the measures of inspection effectiveness to be used in the program.

There is currently no existing data base for evaluating the effectiveness of in-service inspection primary system piping. In the Phase II program, a round robin inspection will provide this data. Section 5.? of this report describes the tests and analyses to be performed. The round rosin will include four materials and six test teams. The inspection effectiveness will be evaluated for minimum code requirements, as-practiced field procedures, and an improved procedure. Inspections will be made under laboratory and simulated fipld conditions with flaws located on both the near and far side of the weld. The round robin inspection will be initiated in December, 1980 and be com jleted by June, 1981. Additional round robin tests will be performed if 1931 on dissimilar metal welds and other selected materials not yet defined.

\subsection{MEASURES OF INSPECTION EFFECTIVENESS}

Because an ultrasonic inspection is such a complicated process, no single measurement can adequately describe inspection effectiveness. This discussion describes three sets of measurements that allow inspection results to be viewed from several perspectives. The measurements include 1) probability estimates, 2) false call rates, and 3) measures of association. We hope that tnis combination of measurements will provide a complete description of inspection effectiveness.

\section{Probability Estimates}

The first set of measurements is a collection of probabilities that describe the code procedure's ability to classify cracks correctly. The most 
important probability in this set is the Probability of Rejection, which is the probability that a particular crack will produce a rejectable indication. A rejectable indication is a crack indication whose dimensions are unacceptably large (that is, whose dimensions (depth, length) are in a specified rejectable range, $R$ ). Because of the organization of the Code procedure, a particular crack can only produce a rejectable indication if the following hierarchy of events has occurred.

$E_{1}$ At least one recordable indication must have been produced by the crack.

$E_{2}$ At least one of the above recordable indications must have been classified as a crack indication.

$\mathrm{E}_{3}$ The dimensions of one of the crack indications must be in the rejectable range, $R$.

These three events can be used to define three probabilities.

RP (Recording Probability) $=\operatorname{Pr}\left(E_{1}\right)=$ probability of at least one recordable indication being produced by the crack.

POD (Probability of Detection) $=\operatorname{Pr}\left(E_{2}\right)=$ probability of at least one crack indication being produced by the crack.

POR (Probability of Rejection) $=\operatorname{Pr}\left(E_{3}\right)=$ probability of at Teast one rejectable indication being produced by the crack.

The above probabilities are assumed to be functions of the true crack depth (d) and are written with depth as an argument (RP(d), POO(d), POR(d)). In reality, all the above probabilities are functions of many more variables than just crack depth. These additional variables; (such as material type, crack shape, crack location, and weld preparation should be considered implicit arguments in these functions. Two probabilities, the Recording Probability and Probability of Rejection, are related to two important continuous variables, ob-response and crack indication size, respectively.

Since an indication on ly becomes recordable when the $d B$-response is above $50 \%$ DAC, a crack can only produce a recordable incication if the maximum d8response from that crack is greater than $-6 \mathrm{~dB}(5 \mathrm{C} \% \mathrm{DAC})$. That is, 


$$
R P(d)=\operatorname{Pr}(r \geq-6 d B)
$$

where $r$ is the maximum $d B$-response from the crack.

A similar relationship exists between $P O R(d)$ and the dimensions of the largest crack indication produced by the crack. If s represents the dimensions of the largest crack indication from a certain crack, then

$$
\operatorname{POR}(d)=\operatorname{Pr}\left(\mathrm{s}_{\sim} \in R\right) \operatorname{POD}(d)
$$

The above formulas suggest that an efficient way to estimate $R P(d)$ and POR(d) may be to determine the distributions of $r$ and $s$ and then use this information to calculate the desired probabilities. Because of these simple relationships, the distributions of $d B$-response and crack sizing error are also useful in characterizing inspection effectiveness.

\section{False Cal1 Rates}

The second set of measurements describes the inspection procedure's false call rates. Three rates can be defined for uncracked material that are analogs to the probabilities previously defined for cracked material.

FRR (Faise Recording Rate) = The expected number of recordable indications that occur in a unit length of uncracked material.

FDR (False Detection Rate) = The expected number of crack indications per unit length of uncracked material.

ROFR (Rate of False Rejections) = The expected number of rejectabla indications per unit length of uncracked material.

The three probabilities and three false call rates describe inspection effectiveness well, but they can be very expensive to measure (particularly the probabilities). Twenty to thirty inspections inust be performed in order to obtain reasonably accurate estimates for the probabilities. During these inspections, no variables that influence these probabilities can be allowed to vary haphazardly. It is sometimes very difficult to perform inspections that do not violate this restriction. Consequentiy, it is useful to have measures of inspection effectiveness that can be estimated from one or two inspections. The following set of measurements is capable of this. 


\section{Measures of Association}

We call the third set of measurements measures of association because these quantities measure the amount of association between an inspection and the true state of the weld. All of these measures of association rely on a vector representation that is capable of describing a weld cross section numerically. Figure 5.1 provides an example of this representation. The circumference of the weld cross section is divided into units of $10^{\circ}$. Eacm $10^{\circ}$ unit of material is associated with a component in the vector, and the i'th component of this vector, $v_{j}$, Jescribes the state of this $10^{\circ}$ unit of material. The component $v_{j}$ is set equal to the maximum crack depth occurring in the $i$ 'th unit of material. If this unit of material is not cracked, $v_{i}=0$.

This vector representation can be used to present the results of an inspection as well as the true state of the weld. The inspection results can be characterized by two types of vectors: recordable indication vectors and crack indication vectors. A recordable indiction vector represents the locations of all recordable indications obtained during the inspection, while a crack indication vector describes the locations of all indications that were classified as cracks.

An inspection's effectiveness in describing the true state of a weld can then be measured in terms of how close the crack indication vector corresponds to its associated true-state vector. Measuring the closeness (or association) between two vectors is a very common mathematical problem with a choice of many different procedures. Here we consider two of the most common measures of association:

1. The correlation coefficient

2. A $X^{2}$ contingency table statistic.

Let the vector $u=\left(u_{1}, u_{2}, \ldots u_{n}\right)$ represent the true state vector and $v=\left(v_{1}, v_{2}, \ldots v_{n}\right)$ a crack indication vector. Both the correlation coefficient and the $x^{2}$ contingency table statistic compare the closeness of component $u_{j}$ to that of $v_{i}$. The interpretation of the two quantities would be most natural if it could be assumed that each of the ordered pairs 


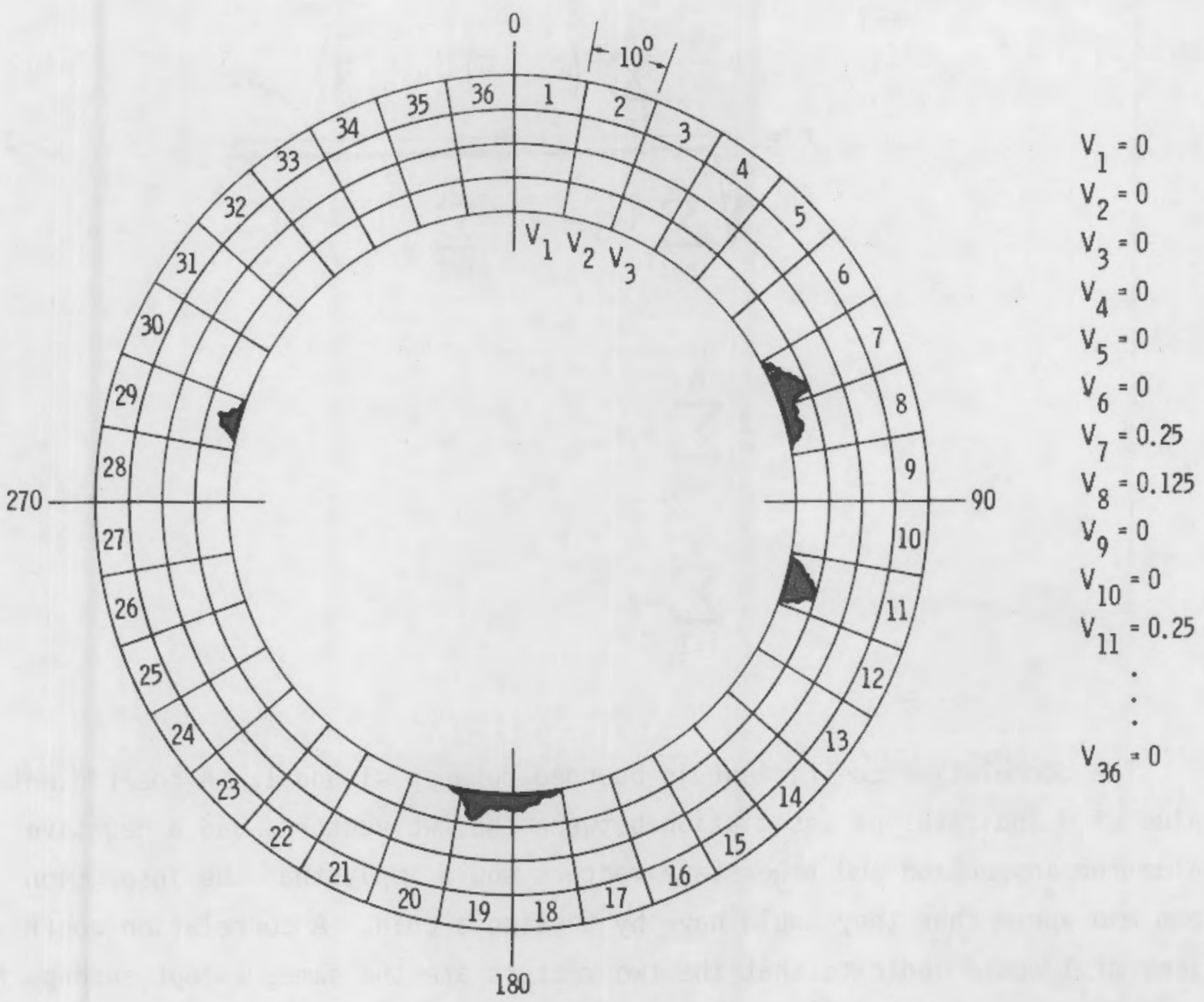

FIGURE 5.1. Vector Representation of a Weld Cross-Section

$\left(u_{j}, v_{i}\right)[i=1 \ldots n]$ came from a particular bivariate distribution $f(u, v)$. If this were the case, both quantities would provide information about the shape of this distribution. 
The correlation between the vectors $u$ and $v$ is defined as:

$$
\begin{aligned}
& r=\frac{\sum_{i=1}^{n}\left(v_{i}-\bar{v}\right)\left(u_{i}-\bar{u}\right)}{\sqrt{\sum_{i=1}^{n}\left(v_{i}-\bar{v}\right) \sum_{i=1}^{n}\left(u_{i}-\bar{u}\right)^{2}}} \\
& \bar{v}=\frac{1}{n} \sum_{i=1}^{n} v_{i} \\
& \bar{u}=\frac{1}{i} \sum_{i=1}^{n} u_{i}
\end{aligned}
$$

The correlation coefficient is bounded between -1 and 1. A coefficient value of 0 indicates no association between the two vectors, and a negative value for inspection and true-state vectors would imply that the inspection team did worse than they could have by tossing a coin. A correlation coefficient of 1 would indicate that the two vectors are the same, except perhaps for an additive and multiplicative constant. A correlation coefficient of 1 would imply that the inspection team had correctly identified the pattern of cracks in the weld but that the recorded depths might be off by an additive and/or a multiplicative constant.

The second measure of association, a $x^{2}$ contingency table statistic, presents a summary of a discretized version of the joint distribution of $\left(u_{i}, v_{i}\right)$. Figure 5.2 presents an example of a contingency table of $u$ versus $v$. The table presents the number of pairs $\left(u_{i}, v_{j}\right)$ that fall in the different cells in the plot record at the top of Figure 5.2. 


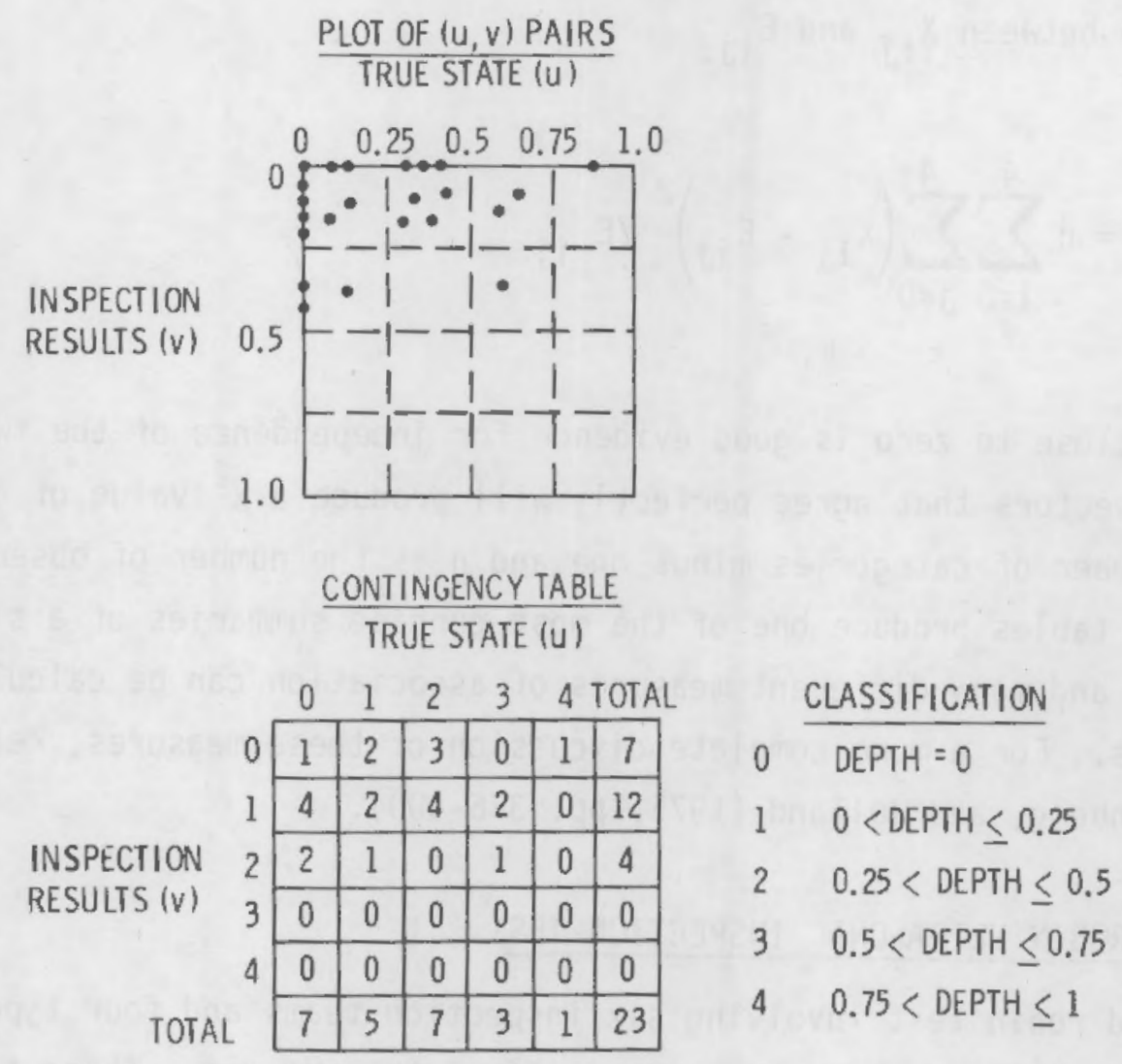

FIGURE 5.2. Construction of a Contingency Table

If the two vectors agree perfectly, only the diagonal entries in the table will be non-zero. If the vectors are independent, the cell counts and their marginals should approximately obey the following relationsin.

$$
x_{i j} \equiv E_{i j}=\left(x_{i+} x_{+j}\right) / n
$$

where $x_{i j}$ is the count in cell ij (row $i$ and column $j$ )

$x_{j+}$ is the total for row $i$

$x_{+j}$ is the total for column $j$

$n$ is the grand total. 
The above relationship can be used to construct a $x^{2}$ statistic that measures the distance between $X_{i j}$ and $E_{i j}$.

$$
x^{2}=n \sum_{i=0}^{4} \sum_{j=0}^{4}\left(x_{i j}-E_{i j}\right)^{2} / E_{i j}
$$

A $x^{2}$ value close to zero is good evidence for independence of the two vectors. Two vectors that agree perfectly will produce a $x^{2}$ value of $4 n$ where 4 is the number of categories minus one and $n$ is the number of observations. Contingency tables produce one of the most concise summaries of a single inspection, and many different measures of association can be calculated from these tables. For a more complete discussion of these measures, refer to Bishop, Feinberg, and Holl and (1975, pp. 376-400).

\subsection{ROUND ROBIN ULTRASONIC INSPECTION TEST}

A round robin test involving six inspection teams and four types of welded pipe samples will be conducted in Phase II of the program. These tests will be initiated in December, 1980. A similar program for dissimilar metal weids will be conducted in 1981 . The objectives of the round robin are to measure the effectiveness of current Code minimum inspection requirements and $t y p i c a l$ field procedures in reliably detecting, locating, and sizing cracks in pipes.

A test matrix has been designed to provide accurate estimates of inspection reliability, as well as the source and magnitude of inspection errors. The measurements and analyses will provide the following information for each procedure:

- Variation between cracks

- Variation between inspection teams

- Variation within inspection teams

- Probability of recording

- Probability of detection

- Probability of rejection 
- Crack location errors

- Crack sizing errors

- Influence of test environment

- Influence of single-side access.

It is not possible to measure the influence of each variable on inspection reliability with any degree of confidence because of time and resource limitations. We will, therefore, fix the test variables at conservative values, in most cases leaving flaw depth as the primary variable. Other values of these variables will be evaluated individually, and their influence on probability of rejection will be calculated in a manner similar to that reported in Section 7 .

In order to provide meaningful information, the tests must be closely controlled and monitored. This effort will require that ultrasonic instruments and search units used by each team be fully characterized, that the teams follow the prescribed test protocol, and that each team receives the same information and opportunity to achieve their best results. The remainder of this section is devoted to addressing these details. The discussion describes the pipe materials and the flaws, the round robin test conditions, the test matrix, and the analysis procedure.

\section{Pipe Materials and Flaws}

Four kinds of pipe will be used in the round robin:

- centrifugally cast 304 stainless steel that has a 32-in. 00 with a 2-3/3-in. wall

- 33-1/4-in. OD of A106 mild carbon steel with a stainless steel ID cladding

- 12-in. schedule-100, type 304 stainless steel

- 10-in. schedule-80, type 304 stainless steel.

These pipes will either be full cylinders or, to ease the handiing of the larger-diameter thick-walled pipe, they will be cut into segments. 
The pipes will contain thermal fatigue cracks, except the 12-in., scheduie-100 pipe, which will contain intergranular stress corrosion cracks (IGSCC). The pipe specimens and the cracks will be thoroughly characterized before the round robin. The cracks will be grown so as to provide the size required by the statistical experimental design. Following the round robin, selected pipe specimens will be destructively anaiyzed to verify the crack dimensions.

The use of thermal fatigue cracks, produced as described in Section 6 , is conservative. The tightness and roughness of these cracks make them difficult to detect as compared to mechanical fatigue cracks. The tightness of these cracks simulate the compressive stresses which may occur during cold shut down, when ISI is performed. The defecting process also results in cracks that are relatively short compared to their depth (less than 5:1 in most cases), which are more difficult to detect.

The pipe specimens have been made by welders qualified to Section IX of the ASME Code. The welds were made under shop conditions but are typical of field practice. Reference marks are located on each pipe specimen to provide a consistent means of locating all indications. The weld crowns were ground flush.

The counterbore configuration is fixed at a conservative, i.e., difficult for inspection, condition. The ground-flush weld crowns are not necessarily a conservative condition. However, they are representative of many Class 1 welds, and allow us to thoroughly evaluate single-side access conditions. The influence of unground and flat-topped weld crowns as well as pipe surface finish will be evaluated separately.

Round Rob in Test Conditions

Discussion of the round robin test conditions can be divided into environmental and general requirements, inspection access, test procedures, and data reporting protocol.

Environmental and General Requirements

There will be two basic environmental set-ups. The first will be a laboratory set-up where the pipe specimen is resting on a table and the inspection 
team can either stand up or sit down while performing the inspection. The second set-up will be a difficult environment although this set-up will still be performed in the laboratory. The inspectors will wear anti-contamination clothing; access to the specimen will be constrained: the inspection surface will be in an overhead position; and the inspector will not be able to see the search unit and the instrument simultaneously.

Testing conditions will simulate field conditions wherever it is possible. The inspectors will be working 12 hour days, six days a week. All the testing will be performed at the Pacific Northwest Laboratory in Richland, Washington, which is away from home for all inspection teams. The teams will have 30 minutes to collect the data from each pipe specimen.

Each pipe specimen will be randomly selected for inspection, but the randomizing will be within blocks of time. In other words, the pipe specimens will be provided in series of the same material and wall thickness which will obviate the need for drastic recalibrations for each pipe specimen.

Numerous pipes will not have any defects and others will contain one or two or more cracks. Again, the selection of the number of cracks in a pipe was based on the experimental design randomization and field-related experience.

\section{Inspection Access}

The pipe specimens will be provided in wooden boxes that prevent the inspection team from having access to the pipe ID. The box provides limited access to the top side of the specimen so that the inspection team will have access to only one side of each weld. This limited access condition is typical of field inspections where most of the welds are from pipe to a component. A typical type of component would be a valve, pump or elbow.

Many component configurations limit access to a single side ( $p$ ipe side). Under these conditions, the weld must be inspected from a single side. If a defect is located on the far side, its response amplitude can be drastically reduced by adverse material properties, particularly by austenitic and dissimilar metal welds. All welds will be inspected assuming single-side access. The test matrix will define the side of the weld on which the crack will oe located, the near side or far side. 


\section{Test Procedures}

There are three sets of procedures that will be evaluated by the round robin. These will be referred to as the minimum ASME Code procedures, the ISI vendor field procedures, and the improved procedures using specified equipment. Each ISI vendor will have a different field procedure, and the improved procedures will be different for each pipe type and each crack type.

The round robin will use a search unit tracking and recording system (SUTARS) where it is possible. The SUTARS data will not be available to the ISI vendor but will be used in establishing the source of errors relative to the results that the vendor reports. If a vendor does not use a Sonic Mark I instrument, then SUTARS position data and flaw detector data will be recorded on strip chart.

Each ISI vendor will be using his normal field procedure to inspect each pipe. The vendor will record the data on his standard forms. However, for reporting the results, a standard form will be employed for ease of scoring. The standard form is discussed in the next section. The data reported on the standard form will be compared to the SUTARS results where possible. This will allow an interpretation of the results as to how or why certain cracks were or were not detected or reported.

The ISI vendors will typically have a more thorough procedure than the minimum called for in the ASME Code Section XI Appendix III. The results for the Code-required inspections will be extracted from the results reported by each vendor. For example, if the vendor reports a crack at $80 \%$ DAC, then according to code, this would not be reported. From this type of interpretation, effectiveness of the minimum code requirements can be established.

Finally, the ISI vendors will be taught new procedures that should enable them to improve their perforinance. The teams will then use these improved procedures on a number of pipes in the round robin matrix to see if they can improve their performance as compared to their first examination. The new procedures are the results of an extensive test and evaluation program on-going at PNL over the past two years. 


\section{Data Reporting Protocol}

The protocol is really the standard reporting format that is to be used by all the ISI vendors. The intent of the format design is to relay all the needed information without burying it in other data. The data required are:

$d_{B}$ - maximum response of the echo signal in decibels relative to $D A C$

$L_{0}$ - circumferential position in inches where the maximum echo occurs

$L_{1}$ - circumferential position in inches closest to the reference mark on the pipe specimen where the signal amplitude is down by $6 \mathrm{~dB}$

$L_{2}$ - circumferential position in inches farthest from the reference mark on the pipe specimen where the signal amplitude is down by $6 \mathrm{~dB}$

$W_{0}$ - axial position in inches relative to weld reference line

$A_{0}$ - axial position in inches from the reference mark to the indication (this is not the transducer position)

TWD - through-wall depth of the crack in $\%$ of wall thickness

DISP - disposition of the data explaining why a recordable indication is being reported or why it is being called a crack or something else

This format should permit easy scoring of the data for input to the statistical analysis computer programs.

Round Robin Statistical Design

The round robin inspections will measure the ability of two variants of the Code procedure to find cracks in four different materials under four different inspection conditions. These factors define 32 conditions that might be measured in the round robin experiment, which are listed in Table 5.1. Because certain sets of conditions are expensive to measure and some are much 
TABLE 5.1. Conditions Under Investigation in the Phase II

Round Robin Ultrasonic Inspection Test

Factor Category

Factor Descriptions

Factor Code

Material Types

10-in. Schedule-80 304 stainless steei

with fatigue cracks

12-in. schedule-100 304 stainless steel

with intergranular stress corrosion cracks

27.5-in. (10) centrifugally cast stainless steel with fatigue cracks

33.5-in. (00) A106 with stainless cladding and fatigue cracks

io-in. SS

12-in. IGSCC

27.5-in. cast

33.5-in. clad

UT Procedure

Current ASME Code Procedure as practiced Code

Improved Code Procedure Improved

Inspection

Conditions

Difficult Field Conditions

Difficult

Laboratory Conditions

Laio

Access

Conditions

Near Side Access--flaw and transducer on Near

same side of the weld

Far Side Access--flaw and transducer on Far opposite sides of the weld

Total Conditions $=4$ Materials $\times 2$ Procedures $x 2$ Inspection Conditions

$x$ ? Access Conditions $=32$ Conditions 
more important than others, all conditions will not be investigated in the same detail. Four experiments of varying size have been selected to investigate the sets of conditions;

1. a full analysis of variance (ANOVA) experiment

2. a truncated ANOVA experiment with replication

3. a truncated ANOVA experiment with no replication

4. a single crack size experiment.

These four types of experiment will be dispersed through the condition set matrix as illustrated in Figure 5.3. The full and truncated ANOVA experiments are located at the most important points in the condition set matrix in a pattern designed to provide some broad information concerning the behavior of the conditions under investigation.

The 10-in. SS material will be most extensively studied, because it is desirable to measure at least one material under all important inspection environments, and $10-i n$. SS is the cheapest material to use for these inspections. The other three materials are measured in detail using a full ANOVA experiment under difficult/near-side conditions. Since measuring the performance of the Code procedure is more important than the performance of the improved procedure, most inspections will utilize the Code procedure.

The inspections using the improved procedure have been included in the round robin to demonstrate the (hopefully dramatic) effect a few simple changes might have on the Code procedure. The improved procedure will be investigated using a truncated ANOVA experiment with all four materials. These truncated ANOVAs will be performed under the same inspection environment as the Code procedure ANOVAS so that the two sets of results $c$ an be directiy compared. The truncated ANOVA should be large enough to allow any important differences between the two procedures to be identified.

Each ANOVA experiment will produce the following information for the investigated set of conditions;

- A dB-respunse curve and a decomposition of the variation around this curve into 3 components of variation 


\begin{tabular}{|c|c|c|c|c|c|}
\hline \multirow{3}{*}{$\begin{array}{l}\text { ULTRASONIC } \\
\text { TESTING } \\
\text { PROCEDURE }\end{array}$} & \multirow{3}{*}{$\begin{array}{l}\text { PIPE } \\
\text { TEST } \\
\text { MATERIAL }\end{array}$} & \multicolumn{4}{|c|}{ INSPECTION CONDITIONS (ENVIRONMENT) } \\
\hline & & \multicolumn{2}{|c|}{ LABORATORY } & \multicolumn{2}{|c|}{ DIFFICULT } \\
\hline & & NEAR ACCESS $F$ & FAR ACCESS & NEAR ACCESS & FAR ACCESS \\
\hline \multirow[t]{4}{*}{$\begin{array}{l}\text { CODE } \\
\text { PROCEDURES }\end{array}$} & $\begin{array}{l}10-I N C H \\
\text { STAINLESS STEEL } \\
\text { (SS) }\end{array}$ & TA & TA & FA & TA \\
\hline & $\begin{array}{l}\text { 12-INCH SS } \\
\text { WITH IGSCC }\end{array}$ & SCS & & FA & TA \\
\hline & $\begin{array}{l}\text { 32-INCH } \\
\text { CENTRIFUGALLY } \\
\text { CAST SS }\end{array}$ & SCS & & FA & TA \\
\hline & $\begin{array}{l}33.5 \text {-INCH } \\
\text { CARBON STEEL + } \\
\text { SS CLADDING } \\
\end{array}$ & SCS & & FA & TA \\
\hline \multirow[t]{4}{*}{$\begin{array}{l}\text { IMPROVED } \\
\text { PROCEDURES }\end{array}$} & 10-INCH SS & & & TAR & \\
\hline & $\begin{array}{l}\text { 12-INCH SS } \\
\text { WITH IGSCC }\end{array}$ & & & TAR & \\
\hline & $\begin{array}{l}\text { 32-INCH } \\
\text { CENTRIFUGALLY } \\
\text { CAST SS }\end{array}$ & & & TAR & \\
\hline & $\begin{array}{l}\text { 33.3-INCH } \\
\text { CARBON STEEL + } \\
\text { SS CLADDING }\end{array}$ & & & TAR & \\
\hline \multicolumn{2}{|c|}{ EXPERIMENT TYPE } & $\begin{array}{l}\text { NUMBER OF } \\
\text { USPECTION TEAMS }\end{array}$ & $\begin{array}{r}\text { NUM } \\
\text { EXPER } \\
\end{array}$ & $\begin{array}{l}\text { MBER OF } \\
\text { RIMENTS }\end{array}$ & $\begin{array}{c}\text { TOTAL } \\
\text { EXPERIMENTS }\end{array}$ \\
\hline \multicolumn{2}{|c|}{ FA - FULL ANOVA } & 25 & & 4 & 100 \\
\hline \multicolumn{2}{|c|}{$\begin{array}{l}\text { TA - TRUNCATED ANOVA, } \\
\text { NO REPLICATION }\end{array}$} & 12 & & 6 & 72 \\
\hline \multicolumn{2}{|c|}{$\begin{array}{l}\text { TAR - TRUNCATED ANOVA, } \\
\text { WITH REPLICATION }\end{array}$} & 16 & & 4 & 64 \\
\hline \multirow{2}{*}{\multicolumn{2}{|c|}{ SCS - SINGLE CRACK SIZE }} & 3 & & 3 & 9 \\
\hline & & & & & $\overline{245}$ \\
\hline
\end{tabular}

FIGURE 5.3. Schedule of Experiments to be Performed Under Phase II Round Robin (condition set matrix) 
- Estimates of crack sizing error and a decomposition of this error into 3 components of variation

- Estimates for the 3 different probability curves, a Recording Probability curve, a Probability of Detection curve, and a Probability of Rejection curve

- Estimates for the 3 different false call rates; a False Recording Rate, a False Detection Rate, and a Rate of False Rejection

- Evaluation of the two measures of association (correlation and the $x^{2}$ statistic) for the inspections performed in the ANOVA.

The three components of variation that will be measured are

- Variation between cracks

- Variation between inspection teams

- Variation within inspection teams.

The only difference between the full and truncated ANOVAs will be their size, so the truncated ANOVAs will produce less precise results than the full ANOVA. However, the single crack size experiment will be too small to produce complete probability curves. That experiment is designed to provide a relatively inexpensive, crude comparison between two sets of conditions.

\section{Round Robin Experiment Structure and Randomization}

The structure of any one of the experiments mentioned previously can be described by a group of indicies $(i, j, k, l)$. Each index in the group describes a factor setting in the experiment. The 4-tuple $(i, j, k, l)$ uniquely identifies a particular inspection to be performed in the experiment and also describes the conditions under which it is to be performed. These indices are particularly useful in distinguishing between the results of different inspections. For instance, the notation $R(i, j, k, l)$ might represent the $d B$-response obtained from inspection $(i, j, k, l)$. The following list describes each of the indices.

- Crack-Size Index: $i=0,1,2,3,4$

This index describes the size of the crack in the specimen under investigation. The actual sizes in the specimens will depend on material type. For example, 10-in. SS will have: 


$$
\begin{aligned}
& i=0--b l a n k \text { specimen } \\
& i=1--10 \% \text { through-wal1 crack } \\
& i=2--25 \% \text { crack } \\
& i=3--40 \% \text { crack } \\
& i=4--55 \% \text { crack }
\end{aligned}
$$

- Specimen Replicate Index: $j(j)=1,2,3,4$

This index uniquely identifies each specimen in the experiment. The index represents the $j$ 'th specimen with a crack of size $i$ in the experiment.

- Team Index: $k=1,2,3,4,5,6$

This index identifies the team that will perform the inspection.

- Inspection Replicate Index, $\ell=1,2$

This index identifies the $\ell-$ th inspection performed by team $k$ on specimen $j(i)$.

Figures 5.4 through 5.7 present a description of the inspections (in matrix form) to be performed for each different size of experiment. Each empty cell in these matrices will contain the results of a single inspection and consequent ly has a 4 -tuple $(i, j, k, l)$ associated with it.

To obtain reliable results from the round robin experiments, it is necessary to control all identifiable sources of variation in the inspection procedures. It can then be assumed that the remaining variation is due to random chance and not to any controllable phenomenon. This is particularly important in these experiments because of the limited number of specimens available for each crack size.

For example, the experiments scheduled in figure 5.3 require that some specimens be inspected seven times by each team. If these seven inspections were all made the same day, the team would certainly become familiar with the size and location of the crack in this specimen. No matter which inspection conditions were imposed, the team would repeatedly record the same response. This would completely undermine the objectives of the experiment.

It is therefore desirable to perform the inspections in an order with no recognizable pattern. This would help to ensure that the teams would make each 


\begin{tabular}{|c|c|c|c|c|c|c|c|c|c|c|c|c|}
\hline $\begin{array}{l}\text { CRACK } \\
\text { SIZE }\end{array}$ & \multicolumn{3}{|c|}{$\begin{array}{l}i=0 \\
\text { (BLANK) }\end{array}$} & \multicolumn{4}{|c|}{$\begin{array}{c}i=1 \\
\text { (10\% THROUGH-WALL) }\end{array}$} & & \multicolumn{4}{|c|}{$\begin{array}{c}i=4 \\
\text { (55\% THROUGH-WALL) }\end{array}$} \\
\hline $\begin{array}{l}\text { INSPECTION } \\
\text { REPLICATE }\end{array}$ & $\mathrm{j}=1 \mid \mathrm{j}=2$ & $j=3$ & $j=4$ & $j=1$ & $j=2$ & $j=3$ & $j=4$ & & & $j=2$ & $j=3$ & $j=4$ \\
\hline$\ell=1$ & & & & & & & & & & & & \\
\hline$\ell=2$ & & & & & & & & & & & & \\
\hline
\end{tabular}

NOTES: 1. THIS MATRIX IS DUPLICATED FOR EACH OF THE SIX INSPECTION TEAMS IN THE ROUND ROBIN

2. EACH EMPTY CELL REPRESENTS A SINGLE INSPECTION. ONLY ONE REPLICATE INSPECTION IS MADE FOR EACH CRACK SIZE.

FIGURE 5.4. Data Matrix for Full ANOVA Experiment for One Team

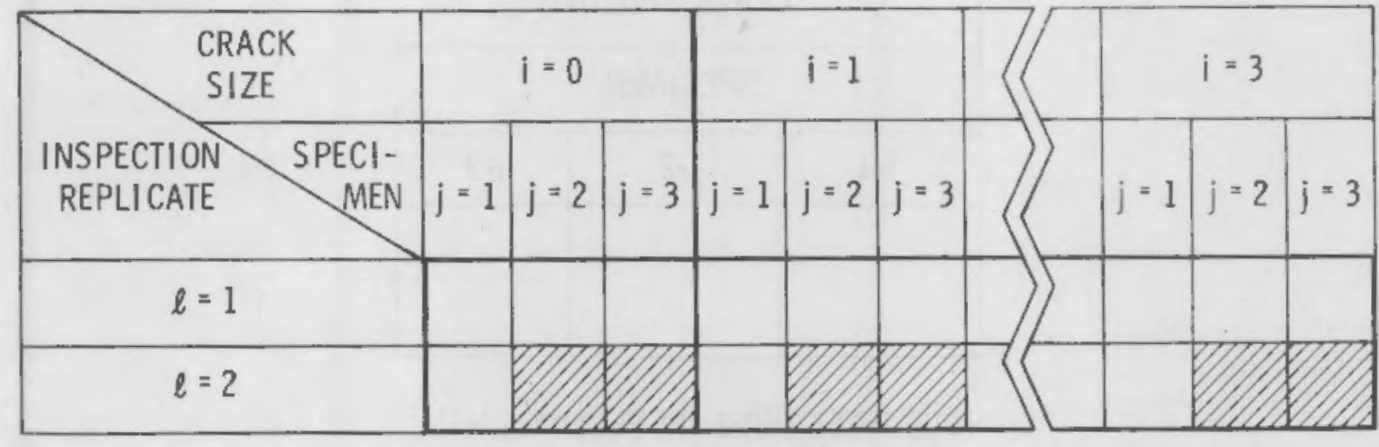

NOTES: I. THIS MATRIX IS DUPLICATED FOR EACH OF THE SIX INSPECTION TEAMS.

2. ONLY ONE REPLICATE INSPECTION IS MADE FOR EACH CRACK SIZE.

3. ONLY FOUR CRACK SIZES ARE INCLUDED.

4. ONLY THREE SPECIMEN REPLICATES ARE INCLUDED.

FIGURE 5.5. Data Matrix for Truncated ANOVA with Replication for One Team 


\begin{tabular}{|c|c|c|c|c|c|c|c|c|c|c|}
\hline $\begin{array}{c}\text { CRACK SIZE } \\
\text { (THROUGH-WALL) }\end{array}$ & \multicolumn{3}{|c|}{$i=0$} & \multicolumn{3}{|c|}{$i=1$} \\
\hline SPECIMEN & $i=1$ & $i=2$ & $i=3$ & $j=1$ & $i=2$ & $i=3$ \\
\hline & & & & & & & \multicolumn{3}{|c|}{$i=3$} \\
\hline & & & & & & & & \\
\hline
\end{tabular}

NOTES: 1. THIS MATRIX IS DUPLICATED FOR EACH OF THE SIX INSPECTION TEAMS

2. THERE ARE NO REPLICATE INSPECTIONS

FIGURE 5.6. Data Matrix for Full ANOVA Without Replication Experiment for One Team

\begin{tabular}{|c|c|c|}
\hline \multicolumn{3}{|c|}{ CRACK SIZE (i=1) } \\
\hline \multicolumn{3}{|c|}{ SPECIMEN } \\
\hline $\mathrm{j}=1$ & $\mathrm{i}=2$ & $\mathrm{i}=3$ \\
\hline & & \\
& & \\
\hline
\end{tabular}

NOTE: THESE THREE INSPECTIONS WILL BE MADE BY EACH OF THE SIX INSPECTIONS TEAMS

FIGURE 5.7. Data Matrix for Single Crack Size Experiment for One Team

inspection independent of previous inspections. It would further ensure that no particular set of inspection conditions would be assigned on "bad" days or during a particular part of the day, etc.

An ideal strategy would be to make a 1 ist of the 245 inspections scheduled for the round robin and then completely randomize their order so that the teams would not be able to recognize any pattern in the order of the assigned inspections. Under such a scheme, every inspection would have an equal chance of occurring on a "bad" day, or at a "bad" time of the day, etc. 
Unfortunately, due to restrictions imposed by recalibration, a complete randomization is not feasible. Inspections will be run in blocks of eight to limit the number of calibrations to two per day. Each block of eight inspections will be performed on specimens of the same material type so that only one calibration need be performed for each block of inspections. The inspections will also have to be blocked by procedure type because the teams must perform the Code procedure inspections before the improved procedure inspections.

To describe a randomization scheme that satisfies the blocking constraints, the 245 inspections are grouped into blocks of (about) eight as described in Figure 5.8. The inspections in a particular procedure/material type combination will be randomly assigned to the associated blocks. For example, the 61 inspections for the 10-in. SS/Code procedure combination will be randomly assigned to eight blocks. Each of the 23 Code procedure blocks will then be randomly assigned a number from 1 to 23 and the eight improved procedure blocks will be assigned a number from 24 to 31 . This assigned number will then be used to determine the run order for these 31 blocks. A new inspection schedule will be generated for each of the six inspection teams by re-randomizing the inspections according to this scheme.

It is expected that a team will be able to perform a block of inspections in a half day. Consequently, a single team should be able to complete the assigned round robin inspections in 15 half days. If in the course of the round robin it becomes apparent that the number of inspections that can be performed in a half day is more (or less) than eight, the remaining inspections can be re-randomized according to a scheme with larger (or smaller) blocks. Round Robin Analysis Procedures

Each of the measures of inspection effectiveness discussed in section 5.1 will be analyzed separately. Most of these measurements will be evaluated using ANOVA models, but Recording Probability, Probability of Detection, and Probability of Rejection will require a different technique; insufficient inspection replications will be run to allow these probabilities to be evaluated with ANOVA techniques. Seven measures of inspection effectiveness will be analyzed using ANOVA Models. They are: 


\begin{tabular}{|c|c|c|c|c|}
\hline $\begin{array}{l}\text { ULTRASONIC } \\
\text { TESTING } \\
\text { PROCEDURE }\end{array}$ & $\begin{array}{l}\text { PIPE TEST } \\
\text { MATERIAL }\end{array}$ & INSPECTIONS & $\begin{array}{c}\text { NUMBER } \\
\text { OF REQUIRED } \\
\text { 1/2-DAY BLOCKS }\end{array}$ & $\begin{array}{l}\text { REQUIRED } \\
\text { TIME, DAYS }\end{array}$ \\
\hline \multirow[t]{4}{*}{$\begin{array}{l}\text { CODE } \\
\text { PROCEDURES }\end{array}$} & $\begin{array}{l}10-I N C H \\
\text { STAINLESS STEEL } \\
\text { (SS) }\end{array}$ & 61 & $\begin{array}{l}5 \text { BLOCKS } \\
\text { (OF } 8 \text { EXPERIMENTS) } \\
+3(\text { OF } 7)\end{array}$ & \multirow{4}{*}{11.5} \\
\hline & $\begin{array}{l}\text { 12-INCH SS } \\
\text { WITH IGSCC }\end{array}$ & 40 & $5(0 F 8)$ & \\
\hline & $\begin{array}{l}\text { 32-INCH } \\
\text { CENTRIFUGALLY } \\
\text { CAST SS }\end{array}$ & 40 & $5(0 F 8)$ & \\
\hline & $\begin{array}{l}\text { 33.5-INCH } \\
\text { CARBON STEEL + } \\
\text { SS CLADDING }\end{array}$ & 40 & $5(\mathrm{OF} 8)$ & \\
\hline \multirow{4}{*}{$\begin{array}{l}\text { IMPROVED } \\
\text { PROCEDURES }\end{array}$} & 10-INCH SS & 16 & $2(0 F 8)$ & \multirow{4}{*}{4} \\
\hline & $\begin{array}{l}\text { 12-INCH SS } \\
\text { WITH IGSCC }\end{array}$ & 16 & $2(0 F 8)$ & \\
\hline & $\begin{array}{l}\text { 32-INCH } \\
\text { CENTRIFUGALLY } \\
\text { CAST SS }\end{array}$ & 16 & $2(\mathrm{OF} 8)$ & \\
\hline & $\begin{array}{l}\text { 33.3-INCH } \\
\text { CARBON STEEL + } \\
\text { SS CLADDING }\end{array}$ & 16 & $2(0 F 8)$ & \\
\hline \multicolumn{2}{|l|}{ TOTALS } & 245 & 31 & 15.5 \\
\hline
\end{tabular}

FIGURE 5.8. Required Number of Half Day Blocks for One Team

1. dB-response

2. crack sizing error

3. $x^{2}$ contingency table statistic

4. correlation coefficient

5. false recording rate

6. false detection rate

7. rate of false rejections

Since each one of these quantities will be analyzed using the same technique,

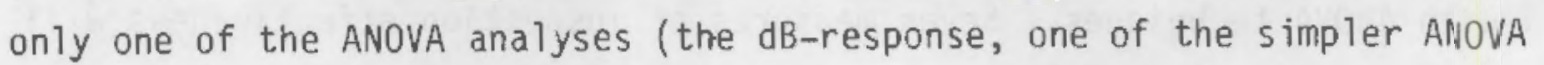
models) is described here in detail: 


$$
\text { Let } R(i, j, k, l)=u(i)+e_{1}(j(i))+e_{2}(k)+e_{3}(i, j, k, l)
$$

where

$$
\begin{aligned}
& u(i) \text { - is the expected } d B-r e s p o n s e \text { from a crack with depth } d_{i} \\
& e_{j}(j(i)) \text { - is the crack replicate effect. This is the variation in } \\
& \text { db-response caused by crack i's deviations in shape, size, } \\
& \text { etc. We assume } \operatorname{Var}\left(e_{1}\right)=\sigma_{1}^{2} \\
& e_{2}(k) \text { - is the team effect. This is the variation in } d B \\
& \text { response caused by differences between teams. We assume } \\
& \operatorname{var}\left(e_{2}\right)=\sigma_{2}^{2} \\
& e_{3}(i, j, k, l)-\text { is the within-inspection error. This term represents } \\
& \text { all variations in } \mathrm{dB}-\text { response that occur randomly (and } \\
& \text { independently) from one inspection to the next. We assume } \\
& \operatorname{Var}\left(e_{3}\right)=\sigma_{3}^{2} \text {. }
\end{aligned}
$$

The above model accounts for three different components of variation which may exist in the data. The analysis of variance will produce estimates for all unknown parameters in the model. Hence, the following estimates will be produced:

$$
\hat{u}(i) \text { for } i=1,2,3,4 \text { (4 points will be produced in a full ANOVA) }
$$

and $\hat{\sigma}_{1}^{2}, \hat{\sigma}_{2}^{2}, \hat{\sigma}_{3}^{2}$.

The three components of variation $\sigma_{1}^{2}, \sigma_{2}^{2}, \sigma_{3}^{2}$ ) should produce one of the most concise descriptions of the weak links in the inspection process. Associated confidence intervals can also be produced for the above estimates, and the four mean responses $(\hat{u}(1), \hat{u}(2), \hat{u}(3), \hat{u}(4))$ can be transformed into a $d B$-response curve using regression. The estimates $c$ an also be substituted into the following formula to obtain a Recording Probability curve:

$$
R P d_{i}=N \frac{\hat{u}(i)-C}{\hat{o} T}
$$


where

$$
\begin{aligned}
N(Z) & =\int_{-\infty}^{Z} \frac{1}{\sqrt{2 \pi}} e^{-1 / 2 x^{2}} d x \\
c & =\text { recording level } \\
\hat{\sigma}_{T}^{2} & =\hat{\sigma}_{1}^{2}+\hat{\sigma}_{2}^{2}+\hat{o}_{3}^{2}
\end{aligned}
$$

The ANOVA results will allow many hypothesis tests concerning the condition factors. These hypothes is tests should be particularly valuable in determining the difference between various material/inspection conditions in the round robin.

The other six measures of inspection effectiveness listed on page 85 will be subjected to an analysis similar to the one outlined above. Some of the ANOVA models used for the other quantities may contain interaction terms, and some of the quantities may be transformed before the analys is is performed. Transformations are used to create a variate whose distribution more closely resembles a Gaussian distribution.

The three probabilities will be estimated directly using dose-response models (see Finney (1964, pp. 437-490) for a complete description of this method). To provide an example of such an analysis, consider the estimation of the Recording Probability curve. Let $x(i, j, k, l)$ represent the success of inspection $(i, j, k, \ell)$ in recording a crack. More precisely:

$$
x(i, j, k, l)=\left\{\begin{array}{l}
1 \text { if inspection }(i, j, k, l) \text { recorded an } \\
\text { indication from crack } j(i) . \\
0 \text { otherwise }
\end{array}\right.
$$

A direct estimate of the Recording Probability for a crack of size $d_{i}$ is

$$
\hat{p}_{i}=\frac{x(i)}{n}
$$


where

$$
\begin{aligned}
x(i) & =\sum_{j} \sum_{k} \sum_{\ell} x(i, j, k, l) \\
n & =\text { total number of inspections perforined under condition } i \text {. }
\end{aligned}
$$

There is evidence to indicate that a curve of the following form should fit the Recording Probability estimates $\hat{p}_{j}$,

$$
p(d / \underset{\sim}{\beta})=N\left(\beta_{0}+B_{1} \ln (d)\right) \quad \text { (Probit Curve) }
$$

where

$$
N(z)=\int_{-\infty}^{z} \frac{1}{\sqrt{2} \pi} \frac{e^{-x^{2}}}{2} d x
$$

$\underset{\sim}{\beta}=\left(\beta_{0}, \beta_{1}\right)$ are unknown parameters

$d$ is crack depth.

To fit the curve to the estimates, an iterative, weighted least-squares technique is used. The least squares technique will produce estimates for the unknown parameters $B$ and also produce the covariance matrix of these estimates. These values can be used to test whether two Recording Probability Curves are different. Other curves could also be $f$ it to the data, but because of the limited number of data points available, only curves containing two unknown parameters can be $f$ it with any accuracy.

The above "direct" method for obtaining a Recording Probability curve should be contrasted to the indirect method presented on page 33 . The indirect method relies on more assumptions than the direct method (normality of dB-response, correctness of the ANOVA Model, etc.). However, the indirect method has the potential of providing more accurate answers. Probabilities will be calculated with both methods and the two methods will serve as checks against each other. 



\subsection{SAMPLE PREPARATION}

The ability of ultrasonic measurements to characterize flaws in metals is limited by the availability of data correlating specific flaw properties with ultrasonic measurements. The objective of this task is to prepare samples with artifically induced flaws (cracks) produced under controlled conditions. Intended to simulate service-induced flaws, the controlled-size flaws provide a basis for correlating ultrasonic measurements with known crack properties.

Two major sample fabrication programs were accomplisined as part of the Phase I program. The first included development of a thermal fatigue technique for production of controlled-sized flaws in complex components not adaptable to conventional fatigue procedures. The second was for the production of cracks with controlled characteristics in flat plate samples by fatigue in four-point bending. Samples from both programs were used in Phase I measurements to determine the effects of flaw roughness and tightness on inspection reliability. The thermal fatigue procedures, developed in Phase I, will be used to produce flawed, welded pipe samples for the Phase II round robin tests. Therinal fatigue cracks are both tight and rough and therefore provide a conservative representation of flaws that might be expected to occur in service. The size and characteristics of the samples produced in Phase I are listed in Appendix $C$ of this report.

\subsection{THERMAL FATIGUE CRACK GROWTH IN COMPLEX STRUCTURES}

Thermal fatigue is a unique technique for controlled crack production, which provides a method for simulating field service flaws in piping systems. The technique's ability to propagate fatigue cracks in predetermined locations typical of field service flaws in complex structures distinguishes it from mechanical fatigue techniques. The freedom to locate the flaws arises from selective loading of the component by localized temperature gradients rather than by bulk loading of the entire part. The abilities to locate the cracks and to control the crack depth and resultant geometry make thermal fatigue a very useful technique of controlled crack production for NOE (nondestructive examination) studies. Controlled cracks produced for the present study were 
the subjects of nondestructive examinations to evaluate the detection capabilities of ultrasonic inspection procedures.

The thermal fatigue technique, which was previously used by General Electric (1), is shown in schematic form in Figure 6.1. The specimen (pipe or plate) is heated to an elevated temperature (e.g., $1000^{\circ} \mathrm{F}$ ), and a local area ( 1.0 in. diameter) on the opposite side of the plate from the heat input is quenched by a water jet. A $0.5 \mathrm{in}$. long by 0.02 to $0.04 \mathrm{in}$. deep notch serves to initiate the crack. The heated specimen is quenched for 5 to $30 \mathrm{sec}$ followed by a water-off period of 25 to $60 \mathrm{sec}$ depending on the specimen thickness and material. During the quench the localized reduction of temperature causes the material to contract; however, the balance of the speciment restrains the contraction, producing high tensile stresses at the starter notch. General Electric (G.E.) was able to grow cracks to depths and lengths on the order of 0.5 in. and 1.0 in., respectively, in low carbon or low alloy steels in thicknesses of 2.0 and 6.0 inches.

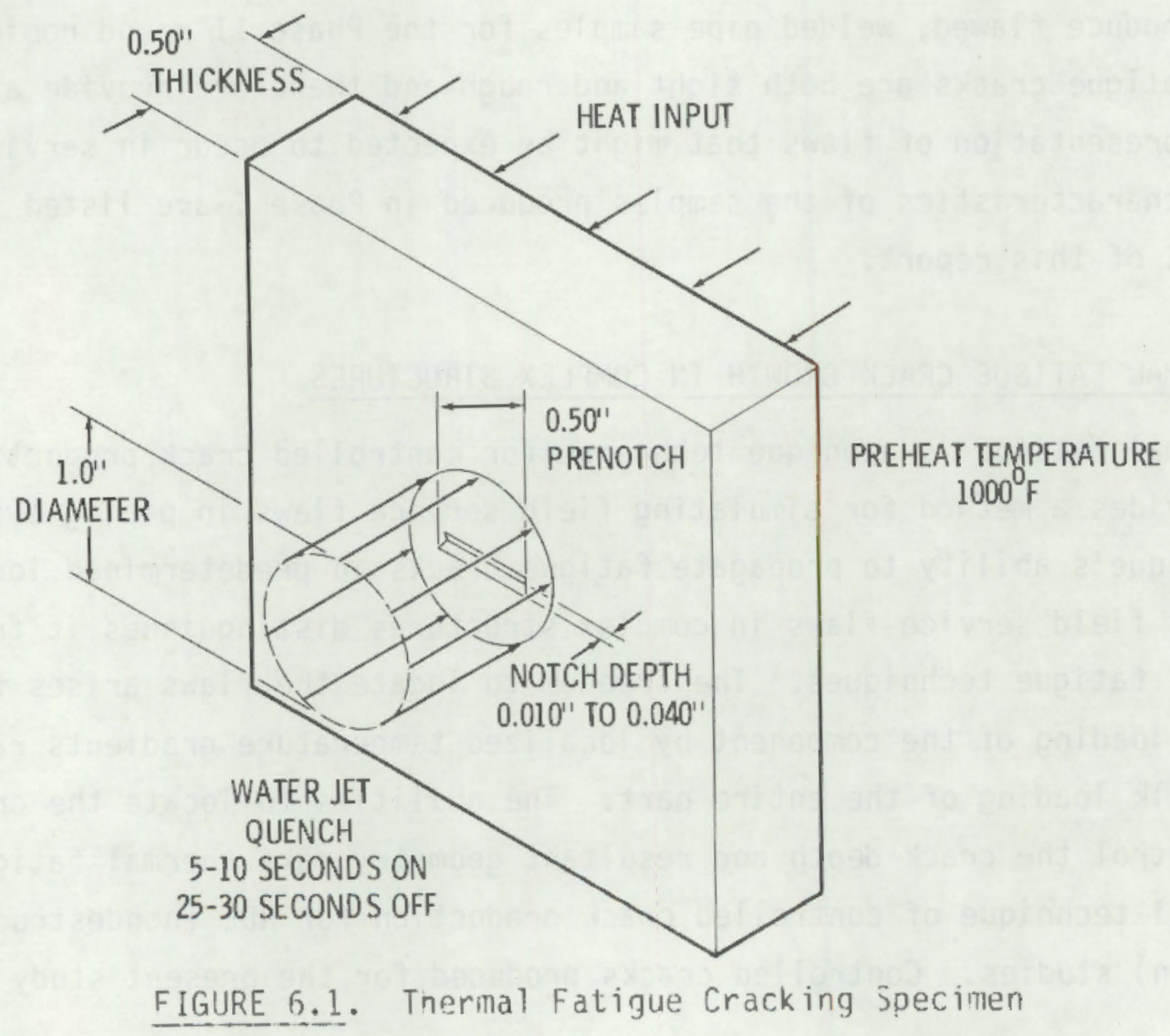


Pacific Northwest Laboratory (PNL) planned to apply the G.E. technique to somewhat different types of specimens. Wall thicknesses as low as $0.5 \mathrm{in}$. were utilized and in some cases a pipe geomet $y$ was used rather than a flat plate. In addition the specimens were stainless steel. An effort to minimize the sensitization of the stainless dictated the use of a preheat temperature less than the $1100^{\circ} \mathrm{F}$ leve) used by G.E. (e.g., $1000^{\circ} \mathrm{F}$ ).

\section{Stress Analysis of Thermal Fatique Crack Growtn}

A study was performed to determine if the thermal fatigue technique could crack the specimens in PNL's prograil witinin a reasonable number of cycles. Approximate stress analyses were performed to predict trends in crack growth rates. The objective was to determine if cracks would grow faster or slower under PNL's conditions as opposed to those of G.E. Details of the study are contained in Appendix 0 . The study assumed that differences due to curvature between a pipe wall and a flat plate were of secondary importance.

The following parameters were selected for investigation; specinen tinickness, cooling time, preheat temperature, and material composition. Within the approximations of the stress analysis, crack depths of interest will be more readily propagated in the thinner $0.5-i n$. specimens than the tinicker plates used by G.E. The analysis also indicated that stainless steels would be suDjected to nigher stress than carbon steels primarily due to the larger expansion coefficient. However, the stainless steels have lower thermal conductivities, which result in a slower increase in the stress intensity factor with quench time. The analysis showed a direct dependence between the crack tip stress and the difference between the preheat and cooled temperature of the quenched surface. Since the crack growth rates are logaritnmic functions of the stress intensity factor, reducing the preheat temperature will have a detrimental effect. Therefore, the preheat temperature must be maintained at maximum practical levels. The study concluded that thermal fatigue cracks could be propagated in PNL's stainless steel specimens.

Feasibility Study of Thermal Fatigue Crack Growth in Stainless Steel

Coincident with the stress analysis, an empirical program was established to determine the feasibility of using thermal fatigue for producing cracks in 
10-in., Schedule-80, 304 stainless steel pipe. A stand-in specimen of 1/2-in. thick 304 stainless steel plate was used in a prototype thermal fatigue system instead of the actual pipe. A block diagram of the prototype thermal fatigue system is shown in schematic form in Figure 6.2. A propane infrared heater located above the plate was used to maintain the top surface temperature of the specimen at $1000^{\circ} \pm 25^{\circ} \mathrm{F}$. A temperature controller was used to regulate the propane flow to the heater using a high/low control regime. A by-pass valve allowed the propane to flow continuously to the heater at a metered rate, while the main solenoid was operated by the controller to provide high heat input. Thermocouples used to monitor the temperature were set in thermocouple.wells to avoid fallacious readings due to the infrared radiation from the heater. The outputs of the thermocouples were recorded on a strip chart recorder for diagnostic and parameter development purposes, as well as to provide a permanent record of the test. The specimen was supported on a 1.25-in.-ID pipe

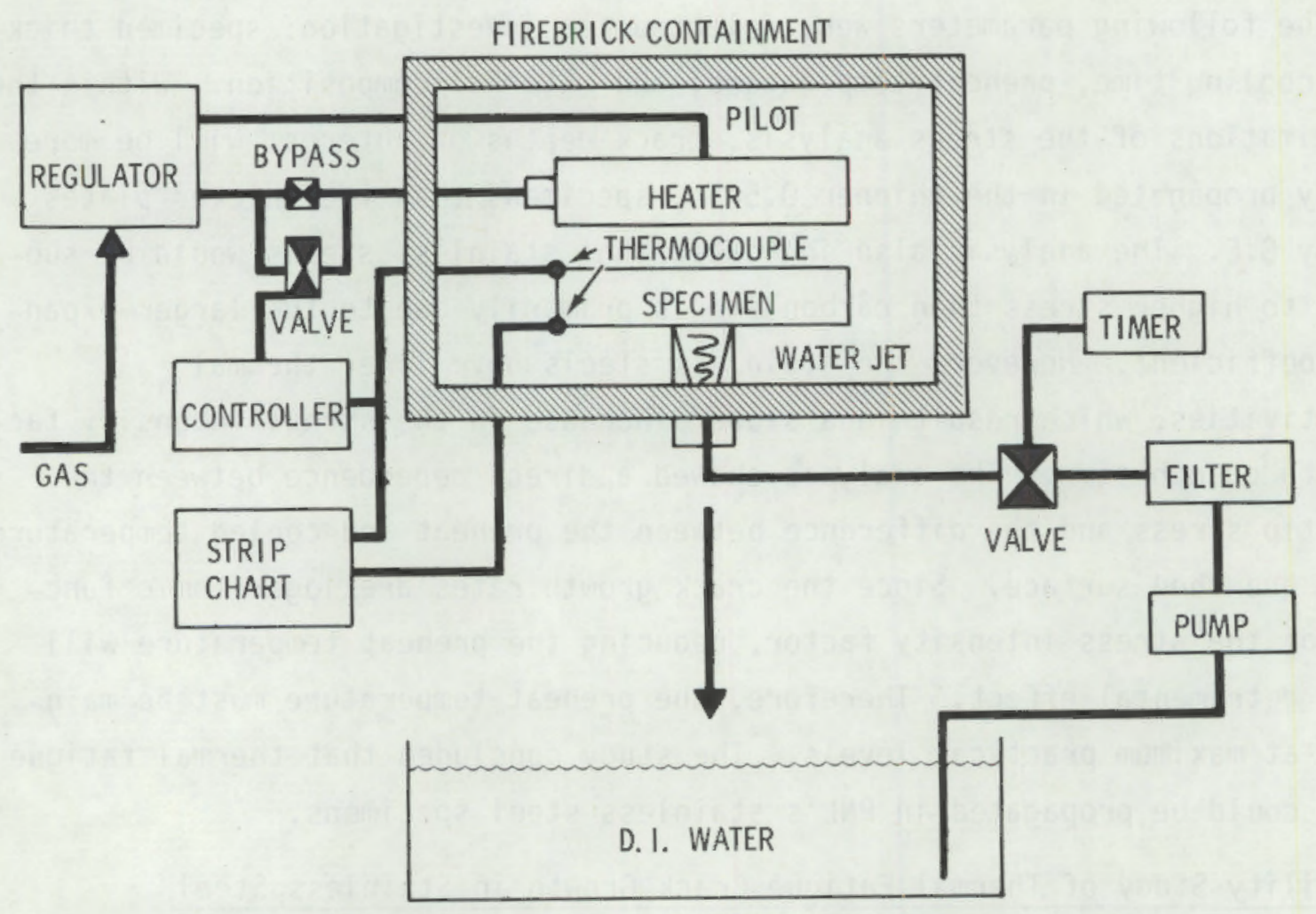

FIGURE 6.2. Thermal Fatigue Facility Block Diagram 
which was used to confine the water jet to a localized area. Distilled water was fed to the water jet on a $10-\mathrm{sec}$ water-on and $30-\mathrm{sec}$ water-off cycle. Distilled water was used to avoid the precipitation of dissolved impurities on the quench surface. A build up of the precipitate would have insulated the specimen from the full severity of the quench. The plate specimen was thermally cycled for a total of 15,000 cycles. The resultant crack is shown in Figure 6.3. The starter notch and the monitoring thermocouple well can be observed on the surface of the crack. The results of the empirical program and the stress analysis showed conclusively that thermal fatigue cracks could be grown in PNL's specimens.

Selection of Thermal Fatigue Procedures Over Mechanical Fatigue Procedures for Pipes

The decision to use thermal fatigue to produce cracks for the Integration of NOE Reliability and Fracture Mechanics Program was based on a comparison of proposed mechanical bend fatigue and thermal fatigue techniques. It was immediately apparent that only the smaller diameter (10-in.) pipes were at issue. The load requirements for the large-diameter pipes would have been very large, requiring the design and development of a costly loading system. On the other hand G.E. had demonstrated the capabilities of thermal fatigue to crack thick materials.

A conceptual design for mechanically producing fatigue cracks in a multiple-weld pipe section is illustrated in Figure 6.4. Up to 10 welds ( 5 in each pipe) could be simultaneously fatigued. The pipes would then be rotated $90^{\circ}$ to $120^{\circ}$ and fatigued again. Three to four cracks could be produced in each weld.

Selection of the thermal fatigue technique to produce controlled cracks in welded pipes was based on the following seven criteria:

1. Feasibility was established by G.E. and PNL on the necessary range of materials and thicknesses.

2. Flaws produced by thermal fatigue were both tighter and slightly rougher than those produced by bending fatigue. These are conservative conditions (i.e., the cracks are more difficult to detect). 


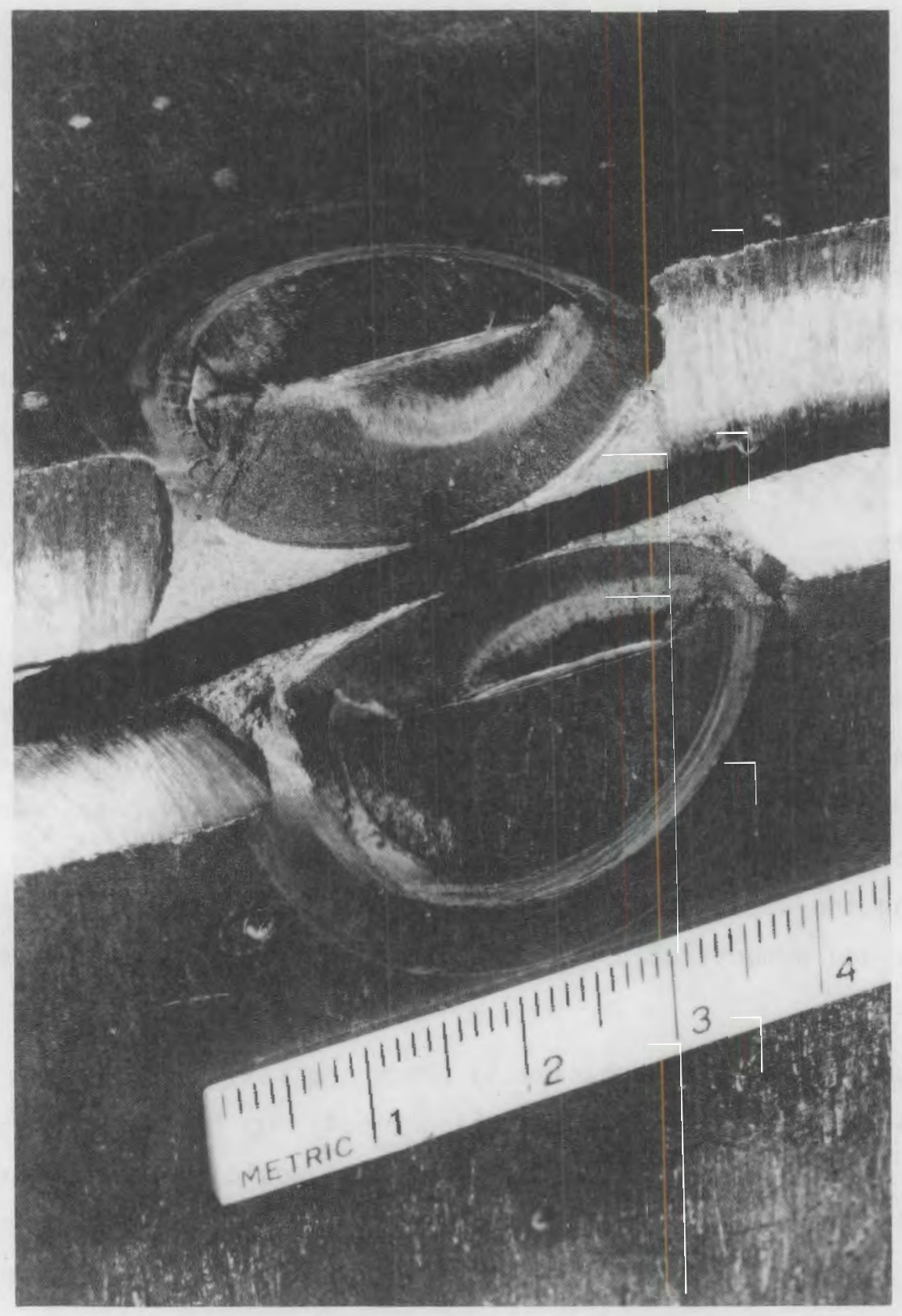

FIGURE 6.3. Thermal Fatigue Crack in 304 SS 


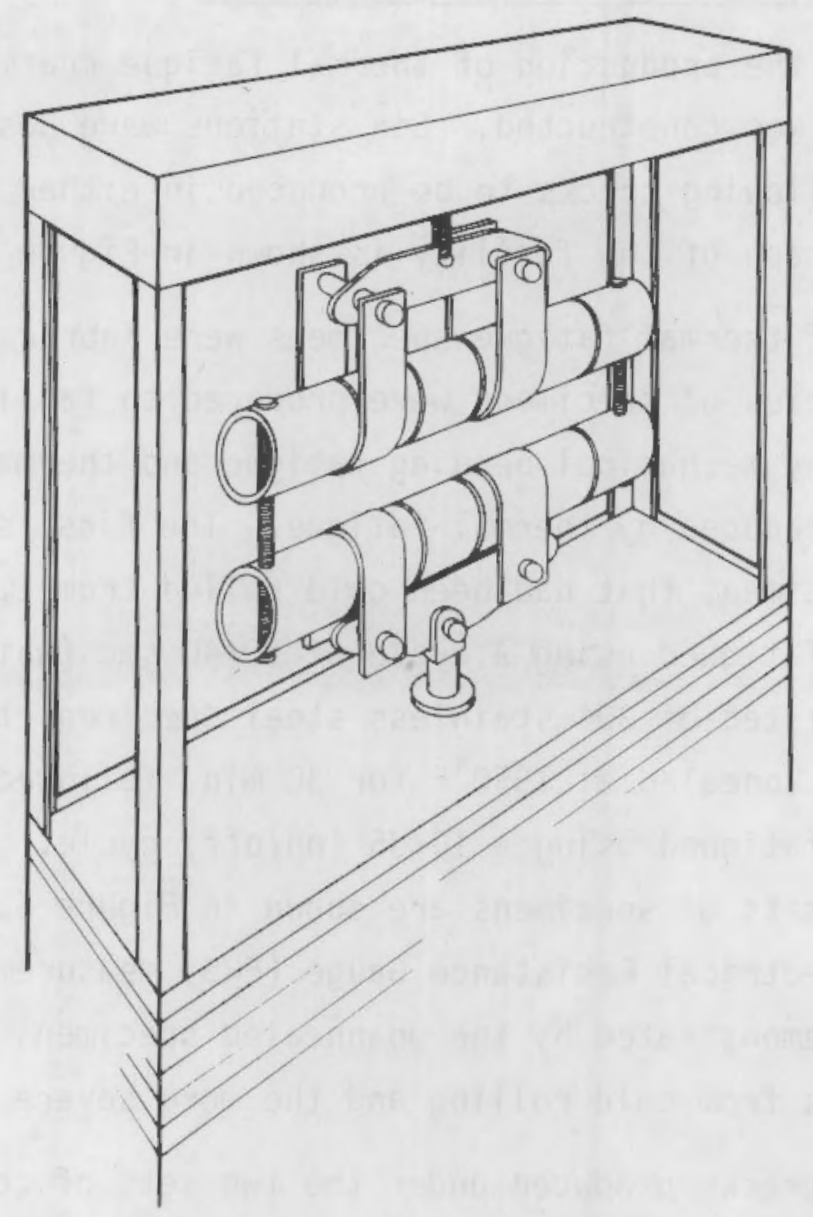

FIGURE 6.4. Mechanical Fatigue Facility for Multiple Welded 10-in., Schedule-80 Pipe (conceptuai design)

3. Small aspect ratios (in the range of 2 to 5) can be produced.

4. The technique is adaptable to large pipe components such as PWR main coolant pipes.

5. Multiple flaws can be produced simultaneously with individual control.

6. Cracks can be loacted in predetermined locations typical of field service flaws.

7. The method is less costly than using large nechanical fatique machines. 
Preparation of Thermal Fatigue Cracking Specimens

To accommodate the production of thermal fatigue cracks needed for the PNL program, a facility was constructed. Six stations were designed for maximum flexibility, each allowing cracks to be produced in either pipe, pipe sections or plate. A photograph of the facility is shown in Figure 6.5 .

Three series of thermal fatigue specimens were fabricated concurrently in 1979. The first series of specimens were produced to facilitate a comparison of cracks produced by mechanical bending fatigue and thermal fatigue. Two sets of specimens were produced by thermal fatigue. The first set consisted of 304 stainless steel specimens that had been cold rolled from $1.0 \mathrm{in}$. to $0.75 \mathrm{in}$. and then thermally fatigued using a cycle of $10 / 40 \mathrm{sec}$ (water-on/water-off). The second set consisted of 304 stainless steel specimens that were cold rolled to $0.65 \mathrm{in.}$, annealed at $1950^{\circ} \mathrm{F}$ for $30 \mathrm{~min}$, followed by an air quench, and then thermally fatigued using a $10 / 35$ (on/off) cycle. The crack propogation data for both sets of specimens are shown in Figure 6.6. The crack depths were obtained by Electrical Resistance Gauge (ERG) measurements. The larger crack growth rate demonstrated by the unannealed specimens can be attributed to residual stresses from cold rolling and the more severe thermal cycle.

The resultant cracks produced under the two sets of conditions were compared with mechanical bending fatigued specimens using destructive examination. The cracks in the unannealed material were nonplanar, apparently heavily influenced by the residual stress in the material. The cracks in the annealed material were more nearly planar, which was typical of previously encountered thermal fatigue cracks.

An important criteria for comparison of the mechanical bending and thermal fatigue cracks is surface rnughness. A profilometer with a 0.001-in. stylus was used to scan the surface at $0.004 \mathrm{in}$. per minute. The profilometer data for the mechanical bending and thermal fatigue surfaces are tabulated in Table 6.1. The roughness measurements indicate that the rough mechanical bending fatigue cracks have approximately the same roughness as the thermal fatigue cracks. This data may be somewhat misleading and it is important to consider its nature. The profilometer data is composed of two components. The first: 


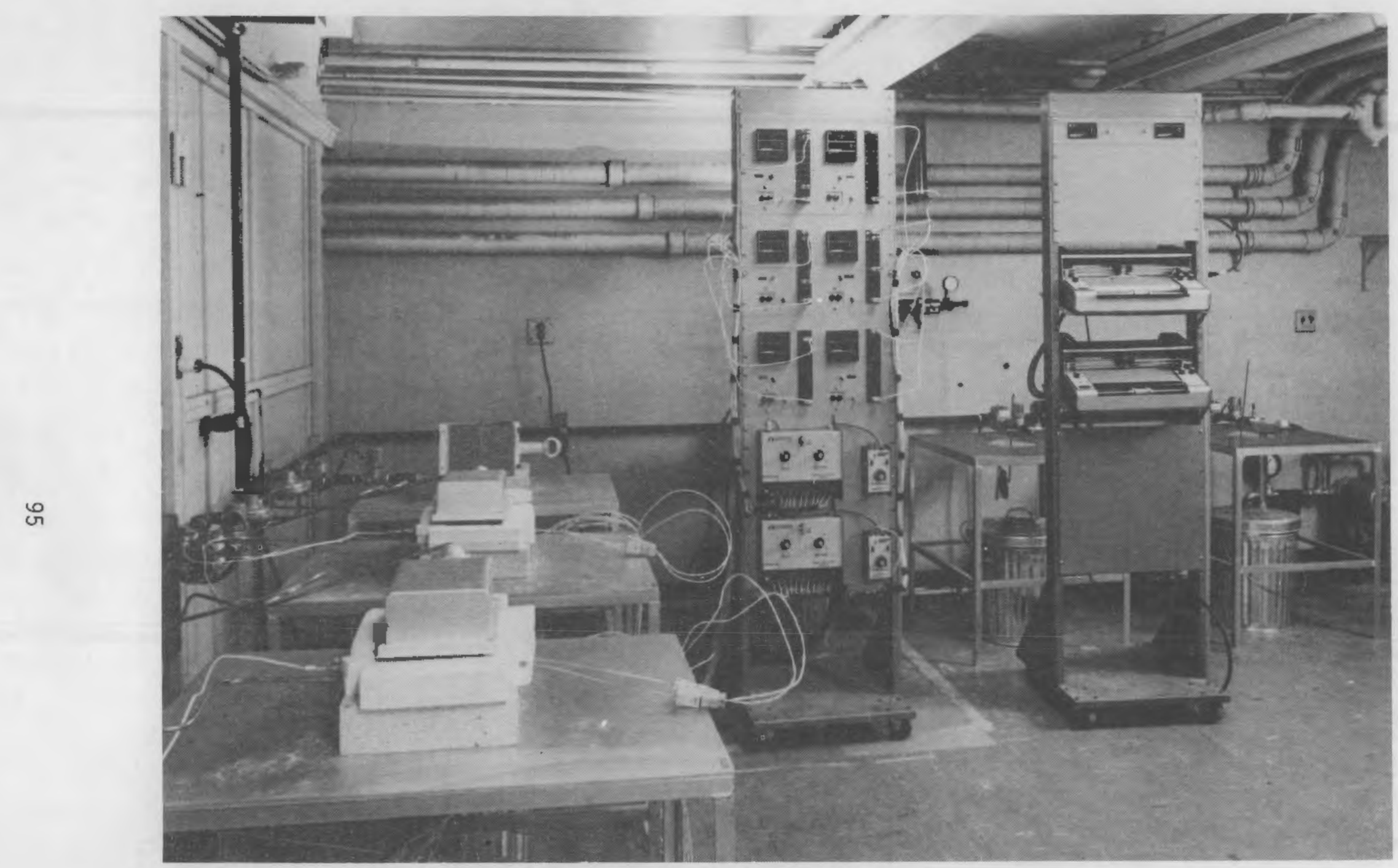

FIGURE 6.5. Therinal Fatigue Facility 


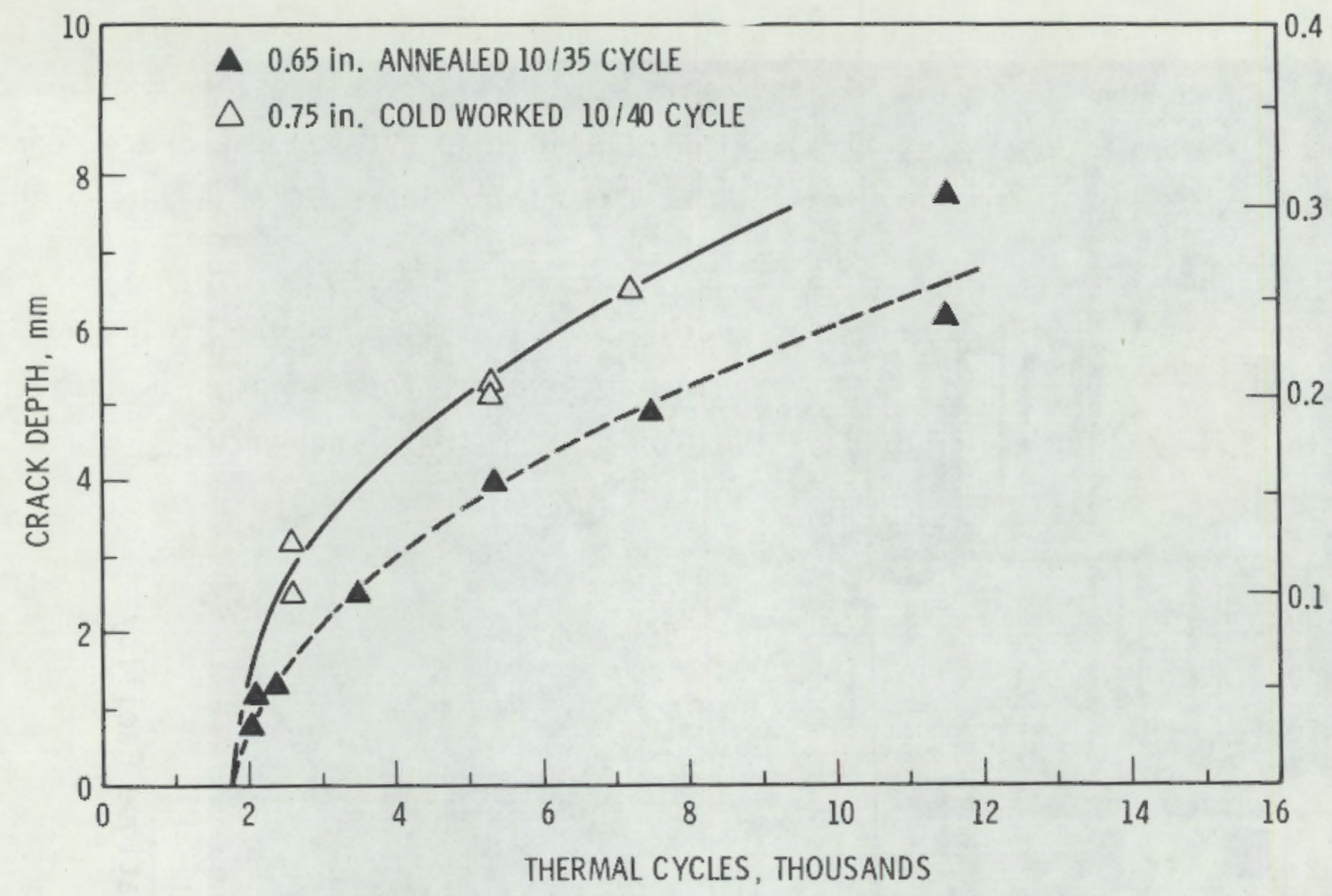

FIGURE 6.6. Crack Propagation of Comparison Specimens

TABLE 6.1. Surface Roughness

\begin{tabular}{|c|c|c|c|}
\hline ecimen & Classification & $\begin{array}{l}\text { Aver age } \\
X(\mu \mathrm{m}) \\
\end{array}$ & $\begin{array}{l}\text { Standard Deviation } \\
S(\mu)\end{array}$ \\
\hline $\begin{array}{l}140 A \\
141 A \\
160 A \\
161 A \\
101 B \\
114 B\end{array}$ & $\begin{array}{l}\text { Rough Mechanical } \\
\text { Rough Mechanical } \\
\text { Smooth Mechanical } \\
\text { Smooth Mechanical } \\
\text { Thermal Fatigue } \\
\text { Thermal Fatigue }\end{array}$ & $\begin{array}{l}7.9 \\
9.2 \\
6.7 \\
5.0 \\
9.3 \\
8.1\end{array}$ & $\begin{array}{l}1.4 \\
3.0 \\
1.8 \\
1.2 \\
2.0 \\
2.0\end{array}$ \\
\hline
\end{tabular}

is a large-amplitude low-frequency component, which is very difficult to measure because it has a floating baseline. The second component is nigher in frequency and smaller in amplitude, being superimposed on the first. It is the bandwidth of this second component which was recorded in the table. Therefore, the thermal fatigue specimens are generally rougher owing to the largeramplitude low-frequency perturbations. However, these are very difficult to measure consistently. 
The second series of specimens were designed to develop the parameters for the 10-in. schedule-80 stainless steel pipe. Stand-in specimens of 0.50-in. stainless steel plate were utilized. The relationship between the crack depth and total number of thermal cycles is illustrated in Figure 6.7. A photograph of the specimens is shown in Figure 6.8 .

Program requirements called for cracks of $10 \%, 25 \%$, and $50 \%$ through-wall depth. The total number of cycles required to produce these deptns is indi.cated in Figure 6.7. Production of these cracks in 10-in. schedule-80 stainless steel pipe will require 2400,5500 and 15,000 thermal cycles, respectively. The thermal cycle for this application consists of a 5-sec water-on and 25-sec water-off period, which results in a frequency of 2 cycles per minute. There- fore, the time required to produce a $50 \%$ through-wall (0.25-in.) crack is approximately 5.5 days.

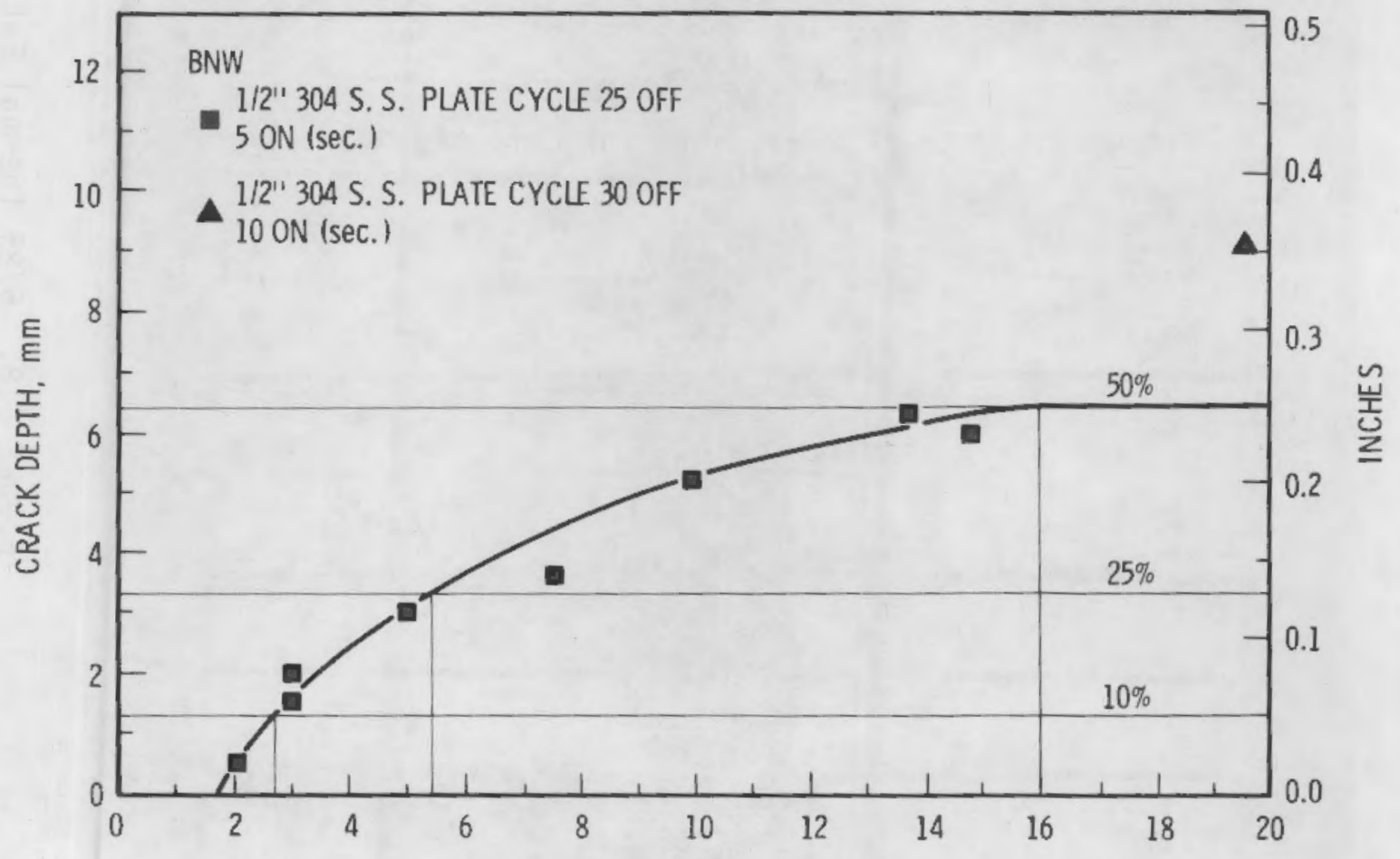

THERMAL CYCLES (thousand cycles)

FIGURE 6.7. Crack Depth vs Thermal Cycles 




FIGURE 6.8. 5/25 Thermal Fatigue Cracks 
The resultant crack geometries as a function of crack depth are illustrated in Figure 6.9. The aspect ratios (crack length/crack deptin) corresponding to the $10 \%, 25 \%$, and $50 \%$ crack depths are $7.5,4.5$, and 3.5 , respectively. An effort to decrease the aspect ratios of the smaller cracks would consist of reducing the diameter of the confining nozzle, forcing the crack to grow into the specimen.

The fixture for thermal fatigue cracking 10-in. pipe was designed and constructed during Phase I of this program. The fixture, which is composed of a spider assembly and three infrared heaters, is shown in schematic form in Figure 6.10. The spider assembly is located in the interior of the pipe near the centerline of the circumferential weld. The three confining nozzles are butted to the pipe by high-temperature springs to provide a good seal with the pipe and secure the spider assembly in the pipe. Water is supplied to the system through the center block and then projected through the spray nozzle. The angles between the nozzles can be changed by simply manufacturing a new center block. Crack production was begun using the first spider assembly in late November 1979. This and subsequent 10-in. pipe specimens will be used in the round robin survey.

The third series of specimens produced in Phase I were in segments of 27-1/2 in. diameter cast stainless pipe. Two cracking stations were set-up for this purpose. Cracks up to $1.0 \mathrm{in}$. in depth have been produced using a 2.5-in.-diameter confining nozzle and a 60-sec heating cycle coupled with a $15-\sec$ quench cycle. It is expected that flaws of the required depth (1.2 in.) for the Phase II round robin samples can be produced using this technique.

\subsection{BENOING FATIGUE CRACK GROWTH IN FLAT PLATE SAMPLES}

In order to evaluate the influence of flaw characteristics (size, shape, roughness and tightness) on ultrasonic inspection reliability, it was necessary to generate a series of cracked samples. Fatigue in four-point bending was selected as the preferred method of crack growth of these samples. The cracks were grown in flat plate, 304 stainless steel specimens to provide a finished sample thickness of 0.6 in. Fatigue parameters were controlled to yield a range of flaw aspect ratios, roughness and depths. The fatigue procedure and results are described below. 


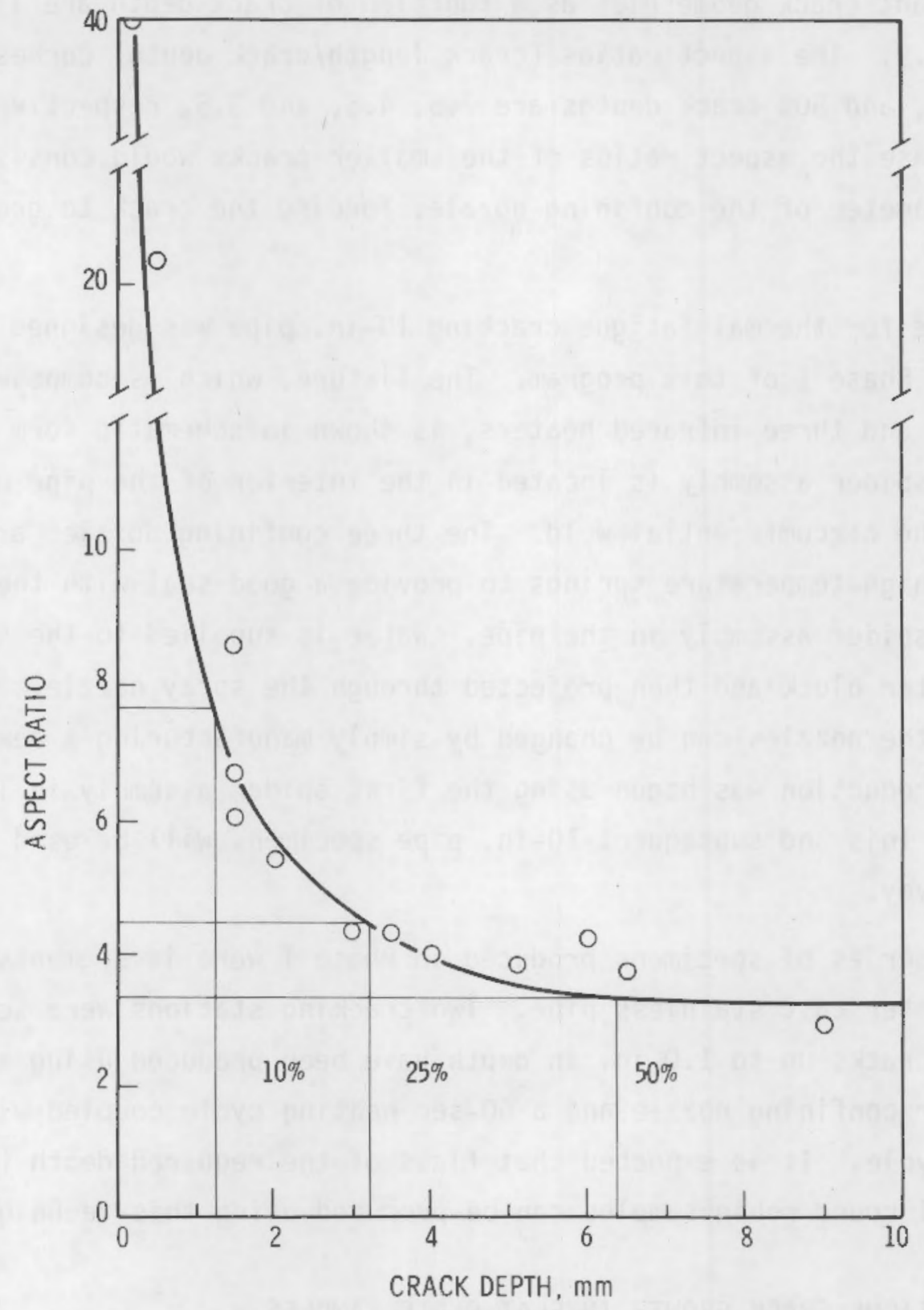

FIGURE 6.9. Aspect Ratio vs Crack Depth

For statistical purposes, the control variables have included the extremes of those likely to be found in structures. This approach should provide cracks that are of optimum use in improving NDE techniques that pertain to actual structural applications. 


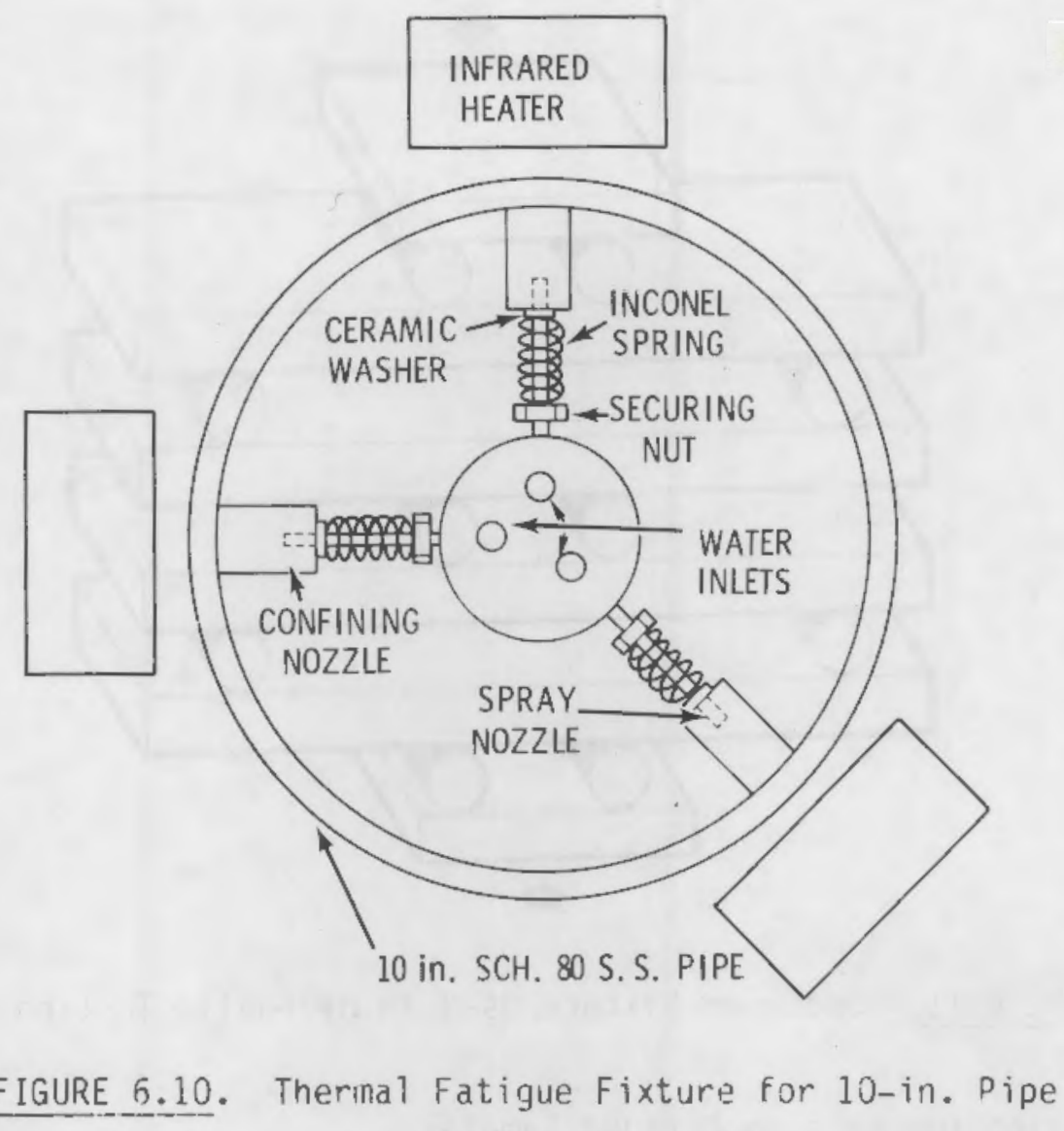

The fatigue test setup for use on a hydraulic test machine is shown in Figure 6.11. This setup was chosen over an available mechanical cyclic test machine because of its speed, ease of setting and adjustment, accuracy, and versatility in the number of specimens to be cycled at a time. The stack of specimens requires no external fixturing. Stack size may be increased or decreased to suit test plan requirements. Crack growth gages are used to detect and monitor crack growth without interrupting cycling.

Grooves at the specimen ends were designed to accept 1 -in. rods. These rods provided adequate clearance so specimens did not contact during loading. Silicon-based adhesive was used at the rod-sample interface to dampen vibrations and maintain specimen alignnent. Aithough the size of the specimen stack was expandable, stack size was either 4 or 2 for all tests. Increasing stack size reduced attainable cycling frequency, because of limited hydraulic flow. Control of sinusoidal waveform, maximum and minimum loads, and frequency was made by closed-loop servohydraulic control. 


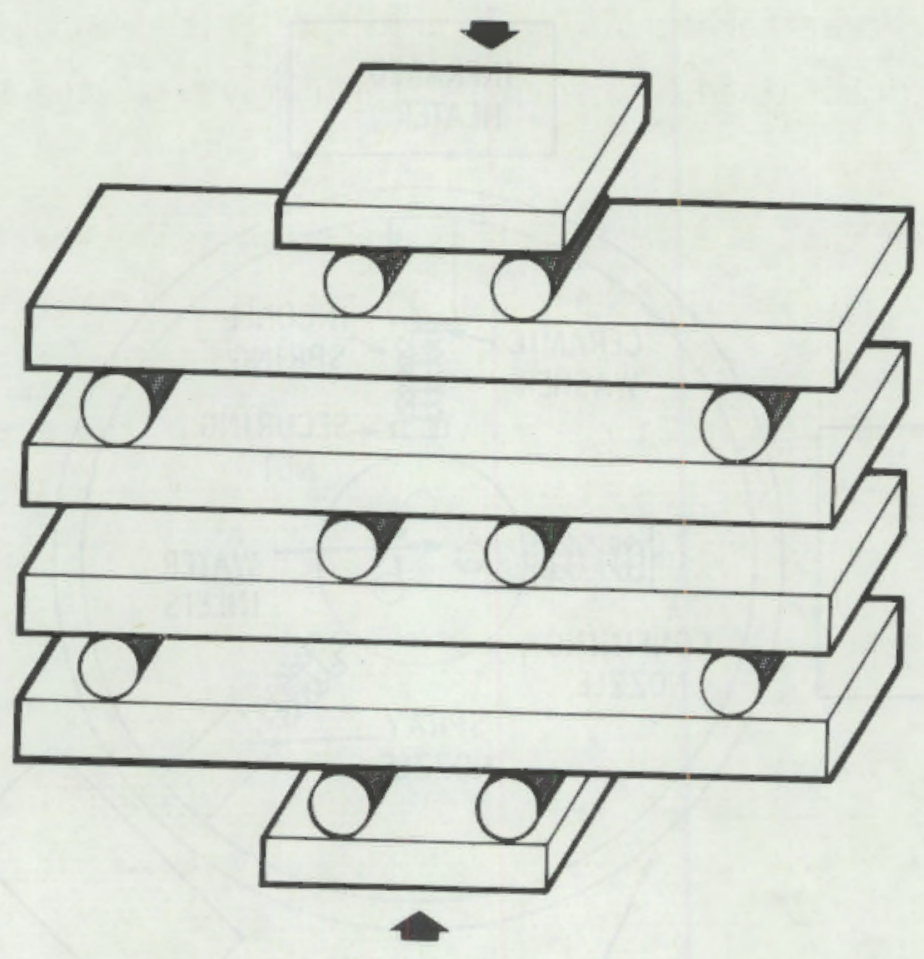

FIGURE 6.11. Specinen Fixture Used in Hydraulic Testing Machine

Crack Initiation for Bending Fatigue Samples

Some type of crack initiator was needed to concentrate stresses sufficiently to cause crack growth to begin in a relatively short time and to locate the growing crack at a desired location. The initiator also influences the shape of the crack, particularly during the early states of crack growth. The initiator must be relatively reproducible and have no influence on NDE signals.

Chemical and mechanical crack initiators were considered. Chemical (crevice corrosion, stress-corrosion cracking) methods lacked reproducibility and shape control; however, they had the advantage of having less influence on NOE signals. Mechanical (sawcut, drilled holes, abrasive-wheel, and electrondischarge machining) methods were more reproducible and controllable, but produced NDE interference. This problem was solved by producing the cracks by mechanical means in over-thickness plate and removing the stress concentrator 
by machining after crack growth. One benefit of this metnod is additional crack shape control obtained by cycling after removal of the stress concentrator.

Abrasive-wheel cuts are satisfactory for long-aspect-ratio cracks, and drilled holes are suitable for short-aspect-ratio cracks. The drilled hole starter consists of three overlapping 1/16-in.-dia holes drilled 0.1 in. deep. Cracks initiate evenly across abrasive-wheel cuts, but those emanating from drilled holes appear to initiate first at the end of the hole, then grow around the sides. This helps to produce low-aspect-ratio cracks.

Bending Fatigue Crack Growth

In a preliminary test, eight specimens were used to evaluate crack shape changes during crack growth. Unlike cyclic tensile loading, cyclic bending produces large changes in crack shape if crack growth is large.

The test indicated it is apparent that three stages of crack growth exist. In the first stage, crack shape remains relatively constant and is primarily influenced by the shape of the crack initiator. This stage is typically 0.1 to $0.2 \mathrm{in}$. for the specimens used in this study. The second stage is a transition stage. Here the initial shape and the stress distribution interact to determine crack shape. The third stage of crack growth is dependent only on the stress distribution. The bending stresses produce crack growth rates higher at the surface of the specimen. As the net section ligament decreases, the surface stress increases, producing acceleration in surface crack growth rate relative to through-wall crack growth rate. Specimens with drilled holes and abrasive-wheel initiators are shown in Figure 6.12 .

\section{Crack Length Measurement}

One of the difficulties associated with crack growth studies is the accurate measurement of crack length. Several methods were tried, including compliance, visual observation of specimen surface, stresscoat, and crack growth gages. 

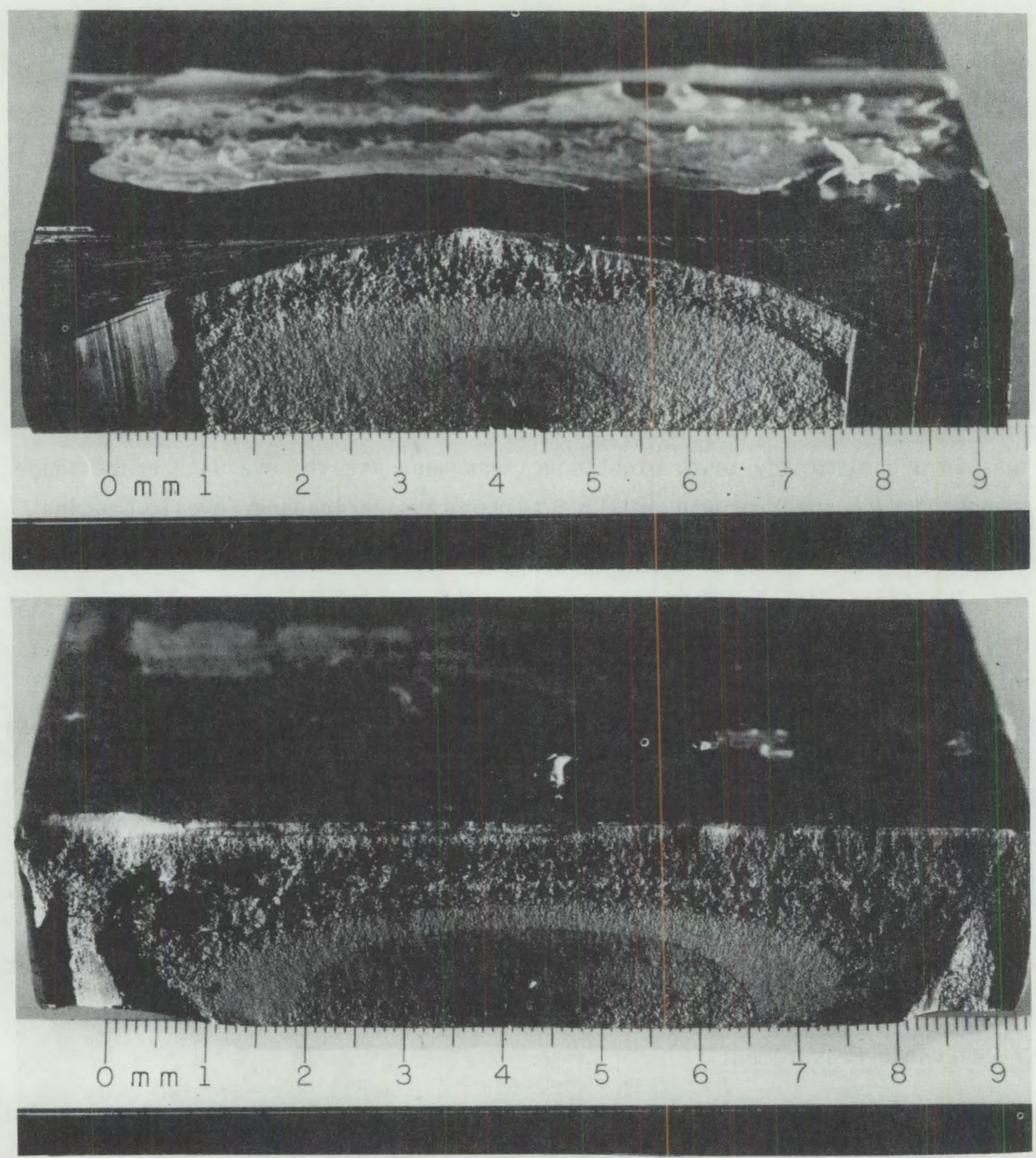

FIGURE 6.12. Crack Surfaces Showing Low-Aspect-Ratio Initiator and Crack (Top) and High-Aspect-Ratio Initiator and Crack (Bottom) (Specimens were broken apart in bending after fatigue cycling. The lower specimen was heat tinted to inark crack size after crack growth was partially completed.) 
Preliminary investigations showed that compliance, as measured by backface strain yage output or by ram-displacement measurements, is not sufficiently sensitive to measure crack lengt or to detect small changes in crack length. Visual observation and measurement of crack lengtir require that the specimen be loaded; however, insufficient clearance exists between specimens for this technique to be useful. Stresscoat applied to the specimen surface helped to locate the crack tip, but the delay associated with unloading the specimens to measure crack length was substantial.

Crack growth gages proved to be the most reliable crack length measurement technique. These commercially available gages use thin strands of wire at even spacing intervals to produce resistance changes as they are broken by the advancing crack. In several instances, the gage was removed to verify that the breaking of wires occurred just as the crack tip crossed the wire. These gages are intended for use in fast fracture, but have produced generally good resuits in this fatigue application. In some instances they have debonded, though, and given erroneous readings.

Control of Crack Aspect Ratios

Control of the aspect ratios of the growing cracks (length/deptn) was conplicated by the use of bending rather than tensile stresses. Surface cracks in tension undergo no dramatic aspect-ratio changes; however, bending stresses increase the tendency for cracks to grow laterally and thus tend toward high aspect ratios (long cracks). This tendency increases greatiy as the crack grows. As the remaining ligament decreases, the surface stress increases, and the fatigue crack growth rate increases exponentially. For this reason, it is easier to produce cracks with high aspect ratios.

To overcome the difficulties of producing low-aspect-ratio cracks, a starter notch was developed which produced rapid crack growth in the through-wall direction and retarded crack growth along the surface. This notch, which consisted of three collinear orilled holes of 0.0625-in. dia and 0.100-in. depth, had a low aspect ratio (2) and was used successfully in all low-aspectratio cracks. An example of this initiator and a low-aspect-ratio crack surface is shown in Figure 6.13 . 


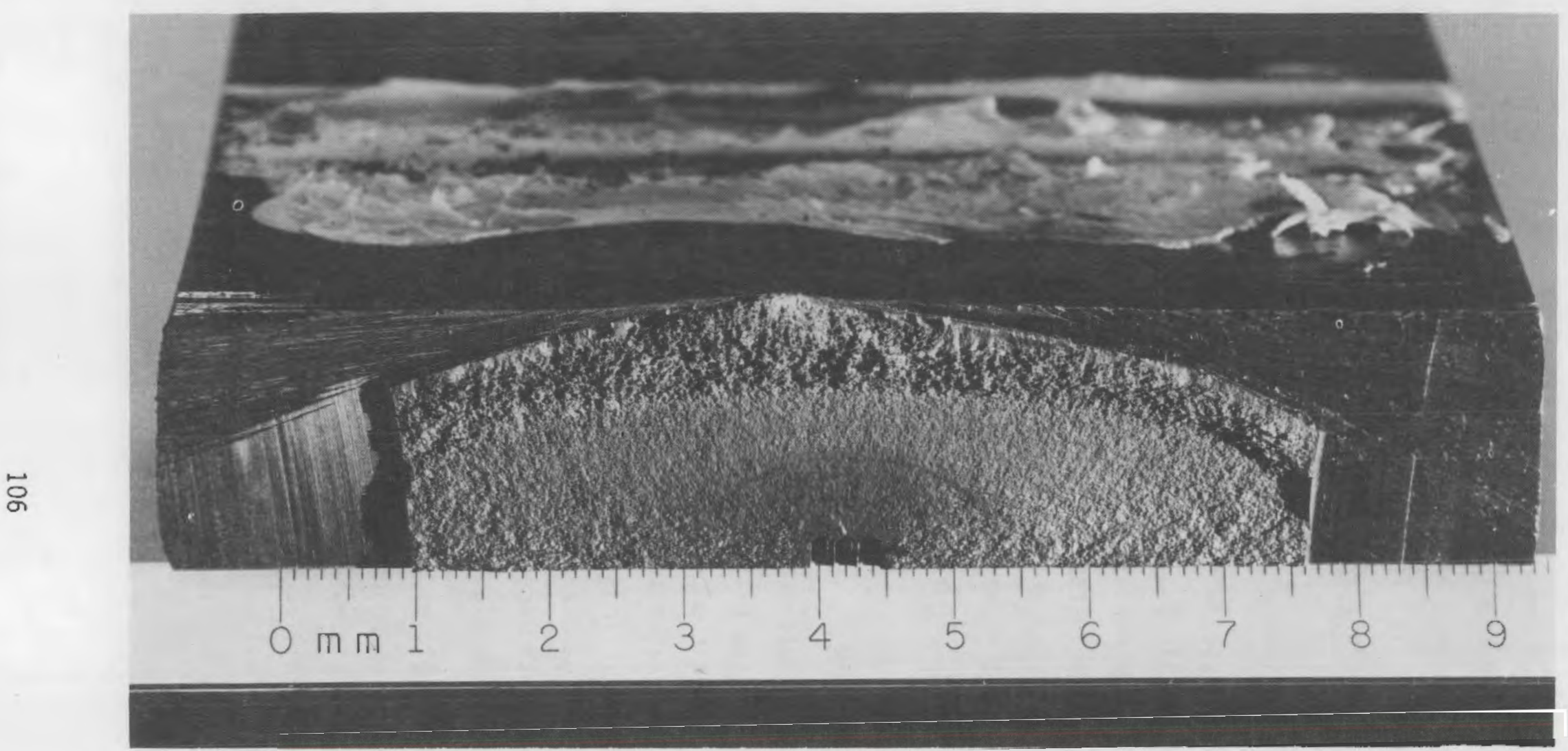

FIGURE 6.13. Expanded View of Low-Aspect-Ratio Crack Face (It is apparent from observation of mariking cycles that the crack initiated at the root of the notch, then grew around the edges of the notch. As crack growth progressed, the aspect ratio increased rapidly.) 
High-aspect-ratio cracks were easier to produce. In this case, abrasivewheel cuts were used as crack initiators. The depth of the cuts was determined using a needle-point dial indicator. Crack initiation occurred at the notch root slightly before it occurred at the surface. The shape of the initiated crack was roughly the same as the shape of the notch in the early stages of crack growth.

Crack Growth Conditions

Cracks of specific roughness characteristics, aspect ratios, and depth were necessary to satisfy the statistical requirements of the program. In addition, it was necessary to produce cracks which encompass the physical characteristics found in actual structures. The array of cracks we intended to make is listed in Table 6.2 .

In Table 6.2, roughness refers to the load at which the cracks were grown. High-roughness cracks were grown at loads 7000 lb (maximum) to 700 1b (minimum). Outer loading pins (Figure 6.11) were 16.0 in. apart; inner pins were $4.0 \mathrm{in.} \mathrm{apart.} \mathrm{This} \mathrm{produced} \mathrm{a} \mathrm{maximum} \mathrm{stress} \mathrm{change} \mathrm{of} 50.4 \mathrm{ksi}$ (high roughness) and $28.8 \mathrm{ksi}$ (low roughness). Stress-intensity changes ( $\Delta K$ ) are dependent on the size of the notch or crack. In all cases, the cracks were initiated at $7000 / 700$ ib and, in the case of low-roughness cracks, the load was lowered at the onset of cracking. An intermediate cyclic load (5000/500 $1 \mathrm{~b})$ was applied to produce a small crack extension and to reduce the number of cycles required. All cracking was done in room-temperature air. Frequency was $4 \mathrm{~Hz}$ for the high loads and $7 \mathrm{~Hz}$ for the low loads.

Crack growth rates vary as the crack grows. Crack growth rates on the order of $10^{-6} \mathrm{in./cycle} \mathrm{were} \mathrm{measured} \mathrm{for} \mathrm{the} \mathrm{low-roughness} \mathrm{specimens,} \mathrm{and}$ rates on the order of $10^{-5}$ to $10^{-4}$ in./cycle were measured for the highroughness specimens.

\section{Results of Bending Fatigue Sample Preparation}

Flaw depths, lengths and aspect ratios are listed in Appendix $C$. The flaw depths after removal of starter notches ranged from 10 to $40 \%$ thickness. Flaw aspect ratios (length/depth) ranged from 4 to 6 , which was somewhat shorter than the desired span. The flaw roughness was in the range of $10 \mu \mathrm{m}$ for both 
TABLE 6.2. Specimen Matrix for Flaw Characteristics Experiment

\begin{tabular}{|c|c|c|c|}
\hline $\begin{array}{c}\text { Spec imen } \\
\text { Number }\end{array}$ & Roughness & Aspect Ratio & $\begin{array}{c}\text { Depth } \\
\% \\
\end{array}$ \\
\hline M139 & rough & 10 & 10 \\
\hline M142 & rough & 10 & 10 \\
\hline M150 & rough & 10 & 20 \\
\hline M154 & rough & 10 & 20 \\
\hline M140 & rough & 10 & 50 \\
\hline M141 & rough & 10 & 50 \\
\hline $\mathrm{M} 147, \mathrm{M} 148$ & rough & 4 & 10 \\
\hline M149, M151 & rough & 4 & 10 \\
\hline M146 & rough & 4 & 20 \\
\hline M144 & rough & 4 & 20 \\
\hline$M 143$ & rough & 4 & 50 \\
\hline M145 & rough & 4 & 50 \\
\hline Ml63 & smooth & 10 & 10 \\
\hline M164 & smooth & 10 & 10 \\
\hline M160 & smooth & 10 & 20 \\
\hline M162 & smooth & 10 & 20 \\
\hline M159 & smooth & 10 & 50 \\
\hline M161 & smooth & 10 & 50 \\
\hline M155 & smooth & 4 & 10 \\
\hline M158 & smootin & 4 & 10 \\
\hline M153 & smooth & 4 & 20 \\
\hline M156 & smooth & 4 & 20 \\
\hline M152 & smooth & 4 & 50 \\
\hline M157 & smooth & 4 & 50 \\
\hline
\end{tabular}

rough and smooth flaws and no difference was noted in the ultrasonic response. Even though the range of flaw characteristics were not as large as desired, the samples have provided valuable information regarding high cycle fatigue flaws 
and the influence of compressive stress on ultrasonic reflection from the flaw. Further investigations will be conducted in Phase 11 on the effects of flaw roughness and crack tightness on inspection reliability. 



\subsection{MEASUREMENT AND EVALUATION}

The primary objective of the Phase I measurement and evaluation program is to identify, measure, and evaluate the inpact of inspection variables which 7 imit the effectiveness of ultrasonic in-service inspection of primary system piping. Recommendations for more effective code and regulatory requirements will be based on these measurements and evaluations. A second objective is to provite estimates of the current level of inspection reliability based on the measured variability of inspections.

Three major sources of inspection variability were investigated in the Phase I program. These included: flaw geometry, flaw character (tightness and roughness), and instrumentation (search unit and instrument). In the flaw geumetry experiments, size, shape, and orientation of the reflector were evaluated as vell as the offects of search unit selection and calioration reflectors. The investigation of flaw character considered the effects of flaw tightness and roughness on the amplitude of reflected ultrasonic signals. The investigations concerning the influence of search unit and instrument operating characteristics on inspection reliability are only preliminary. However, they indicate that considerable variations in inspection results can occur due to instrumentation operating characteristics. The investigation of inspection variables is continuing and will be extended to include the influence of caliJrdtion reflectors, diameter and thickness effects, surface roughness and 00 profile, attenuation and velocity differences between calibration samples and pipe, single-side access conditions, and scan pattern overlap. Based on the results of these evaluations, as well as on the results of the round robin tests (Section 5.?) recommendations for improved code and regutatory inspection requirements will be developed and recommended.

Estimates of inspection reliability are needed by organizations performing probabilistic fracture mechanics for use in failure analysis. There is currently no available data base on which such estinates could be based. Estimates made by inspectors have been shown by the "Plate inspec:ion Steering Comnittee" (PISC 1979) to be highly optimistic. This program has been requested to make these estimates based on measured variabilities of the 
inspection process. A probabilistic model for astimating inspection reliability has been developed for this purpose. The model is based on measurements made as a part of this program. The predicted reliabilities are in close agrement with similar estimates made by Silk (1978) and Forli (1979).

\subsection{EFFECTS OF FLAW GEOMETRY ON INSPECTION RELIABILITY}

The objective of these flaw goemetry measurements is to define the influance of flaw size and orientation on the utrasonic response from the flaw and its impact on detection reliability. A second oujective is to assess the impact on detection reliability of uncontrolled search unit selection (size and operating characteristics). These measurements have been carried out asing electro discharge machined (EDM) notches and saw cut notches in 304 stainless steel plates. The notches s, ulate flaws located on the counterbore (angled back surface), nonvertical crack growth, and nornal vertical crack growth. Notch Samples Prepared to Model Cracked Pripe

The ultrasonic test samples were fabricated to model 10-in., Schedule-30 pipe. They are 304 stainless steel bars, 0.6 in. thick to represent the actuai pipe wall thickness. The difference in ultrasonic response between these flat samples and actual curved pipe (scanned axially) increases with the number of surface reflections. This difference in response will not be negligible for a $3 / 2 \mathrm{~V}$ path, but may be negligibly small for a $1 / 2 \mathrm{~V}$ path. Subsequent research with EDM notches in actual pipe samples will establish a working relationsnip between data taken from these samples and data taken in pipe inspection.

The EDM notches were made to resemble actual cracks witn their widtin held below $0.010 \mathrm{in}$. The notch profile is curved, as if a coin had been pressed edge first into the metal and then removed. The aspect ratio, notch length divided by depth, is 5 . The saw cuts, 0.020 in. wide, have aspect ratios as close to 5 as possible with a limited set of blade diameters. All fall in the range 4.5 to 6.0 .

Normal vertical notches simulate cracking found near the root of a weld. They are cut into bars, 4 in. by $12 \mathrm{in.} \mathrm{with} \mathrm{their} \mathrm{length} \mathrm{centered} \mathrm{along} \mathrm{the}$ 4-in. dimension. The depths are 20,40,60,80,100,120, 160,200, 240 and $300 \mathrm{mils}$. 
Nonvertical notches simulate crack growth along the weld fusion line. These notches have through-wall penetrations of 100,200 , and $300 \mathrm{mils}$, and angles from vertical are $0^{\circ}, 2.5^{\circ}, 5^{\circ}, 7.5^{\circ}, 10^{\circ}, 15^{\circ}, 20^{\circ}, 25^{\circ}$, and $30^{\circ}$. In all other respects these samples are similar to the normal vertical notches.

Vertical notches in angled surfaces simulate vertical crack growth in the counterbore region; the cracked (notched) surface is angled, but the flaw is nornal to the opposite surface. Notches were cut into samples with back surface angles of $5^{\circ}, 10^{\circ}, 15^{\circ}$ arid $20^{\circ}$. Each notch is located in the plane of $0.5-i n$. thickness with deptins of 100,200 , and 300 mils.

The calibration sample is also of 0.6 -in.-thick 304 stainless steel. It contains 1-in.-long 5\%, 10\%, 20\% and 30\% notches; the $10 \%$ notch is per ASME Boiler and Pressure Vessel Code Section XI, Appendix 3, 1977. Alsn, a 2.-in. deep, 3/32-in. side-drilled hole was fabricated at one half the sample thickness (1/2 t) per ASME Bdiler and Pressure Vessel Code Section V, Article 5, Section T-533, 1977.

\section{Transducers Used and Measurements Made}

Each notch was inspected with several search units and beam patns typical of field practice. The peak amplitude of reflection was recorded for each notch.

Transducers used in these tests included:

- 0.25-in.-dia, 2.25-MHz Aerotech Gamma

- 0.5-in.-dia, 2.25-MHz Aerotech Gamina

- 1-in.-dia, 2.25-iMHz Aerotech Gama

- 0.5-in. by 1-in., 2.25-Mz Aerotech Gamma

They were used in $1 / 2 \mathrm{~V}$, full $\mathrm{V}$, and $3 / 2 \mathrm{~V}$ beam paths at a $45^{\circ}$ Deam angle, and in $1 / 2 \mathrm{~V}$ path wits a $50^{\circ}$ bean angle.

The altrasonic instrument presently in use is a Sonic Instruments Mark I, but many measurements will be repeated using a Nortec 1310 instrument.

Peak amplitude of reflection was measured with each transducer and each beam path, from both directions, for a total of 32 measurements per notch. The angied notches and the notches in angled surfaces were inspected from botn 
sides because the reflection geometry is direction dependent. The straight notches were inspected from both sides because some of them were fabricated with a slight, unintentional curvature. That is, the vertical plane of the notch was curved a bit. This had a focusing effect when inspected from one direction (concave reflector) and a dispersing effect when inspected from the other direction (convex reflector). The difference in amplitude of reflection varied from a zeroor one-decibel difference to as much as five or six decibel; in a few cases. The reflection amplitudes in the two inspection directions have been averaged to eliminate the influence of this unwanted curvature. The samples with angled back surfaces were inspected with $1 / 2 \mathrm{~V}$ paths only.

Peak amplitudes were measured in decibels relative to DACs established from the calibration samples. This permits comparison of response between different transducers and beam paths.

Flaw Geometry Measurement Results

The flaw geometry measurement results define the influence of flaw deptin and orientation on ultrasonic response amplitude. The results are presented in seven sections:

- Normal Vertical flaws

- Angled Notches and Vertical Notches in Angled Back Surfaces (measurement results for flaws of $100-m i l$ depth)

- Angled Notches (measurement results for all deptis!

- Vertical Notches in Angled Back Surfaces (measurement results for all depths)

- Notch Detection in Simulated Thicker-Wall Pipe (effect of increasing the wall thickness)

- Comparison of Code Calibration Reflectors (effect of calibration geometry on inspection sensitivity)

- Summary: Impact of Flaw Geometry Results on Inspection Reliability. 


\section{Normal Vertical Flaws}

The results of measurement from normal vertical flaws (slits) appear in Figures 7.1 through 7.4. The curves exhibit, as one would expect, monotonically non-negative slopes of amplitude versus slit depth. For low slit depths, the slopes are high. The fraction of the beam intercepted by the largest slits approaches unity, so the slopes are low. As slit size surpasses the cross sectional area of the beam, the reflection amplitude reaches its maximum and further increases in slit size produce no effect on amplitude. This zero slope condition (infinite reflector) is illustrated by the 0.25-in.- dia transducer; slit depths of $0.160 \mathrm{in}$. or greater all produce the same reflection amplitude. Note that this transducer does not reach the zero slope condition in a $3 / 2 \mathrm{~V}$ path until slit depth reaches $0.200 \mathrm{in}$. because the long path produces a larger beam cross-section area. The largest transudcers have the broadest beams, so they give the highest slopes for deep slits. For shallower slits, the smaller transducers give higher amplitude reflections than large transducers, because the slits intercept a larger fraction of the beam area. As slit depth increases, the amplitude for large transducers is still increasing, so the large transducers give higher amplitudes for deep slits. For the 0.25-in.-ndia and 1-in.-dia units, the crossover point (slit depth for equal reflection amplitude) is about $0.160 \mathrm{in}$. For the 0.5-in. and $1-i n$. units, crossover occurs at about 0.189 in.

\section{Angled Notches and Vertical Notches in Angled Back Surfaces}

Initially, these flaws were machined with only the 100-mil depth. Unexpected results led to the fabrication of 200-mil and 300-mil notch sets.

The test resuits from the angled notches and notches in angled back surfaces with 100-mil depth are shown in Figures 7.5 through 7.10 . These results (at first impression) may appear confusing and less drastic than one would expect from purely geometrical considerations.

However, most of the features displayed are explainable, at least qualitatively. Features of note inciude the following: 


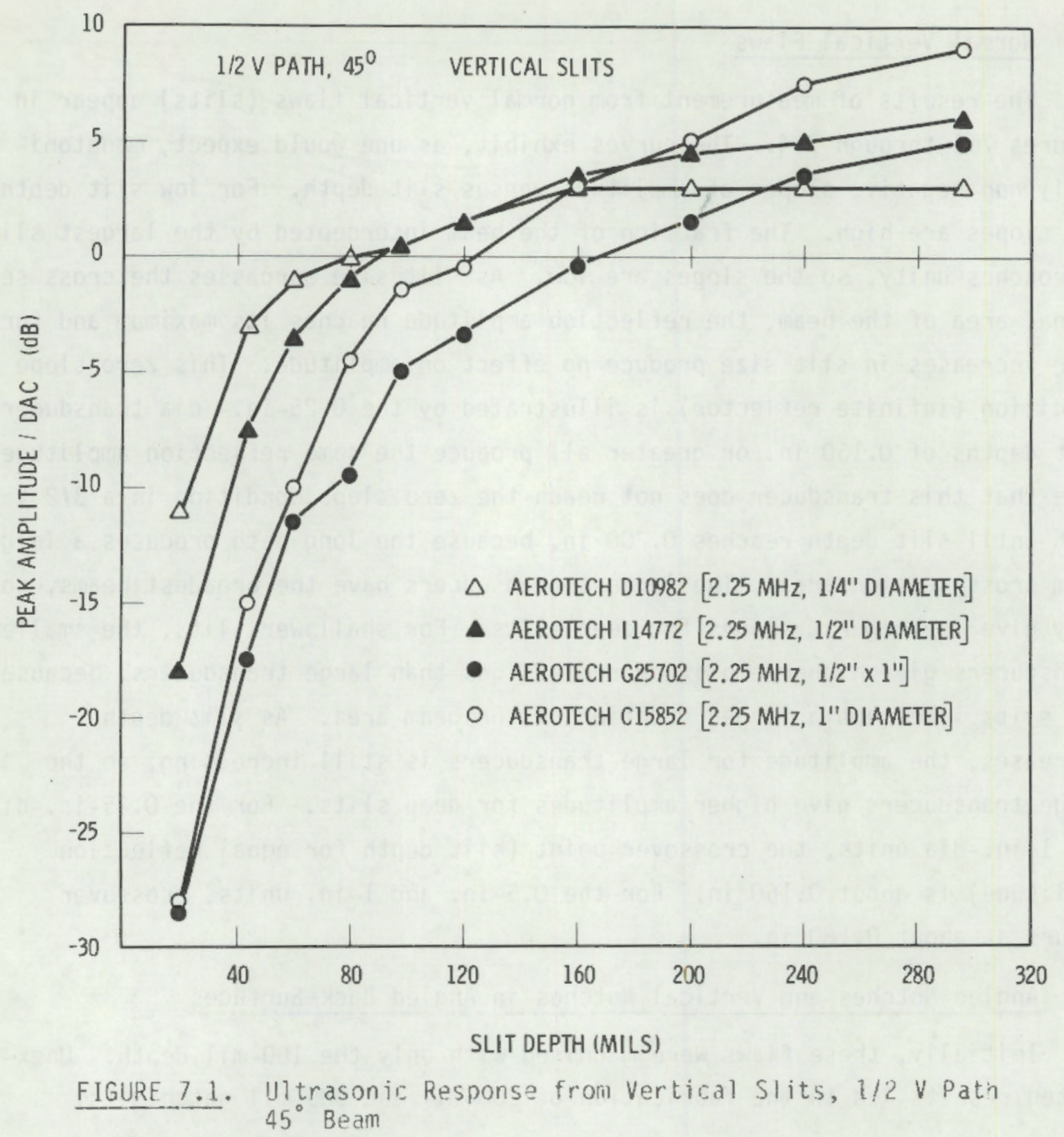

1. The four different search units follow the same basic trend. This indicates the effects are real rather than an artifact of the search unit.

2. The 1/4-in. unit appears in general to be less severely influenced by geometrical effects. This is logical since it has the largest beam spread of the four units tested. 


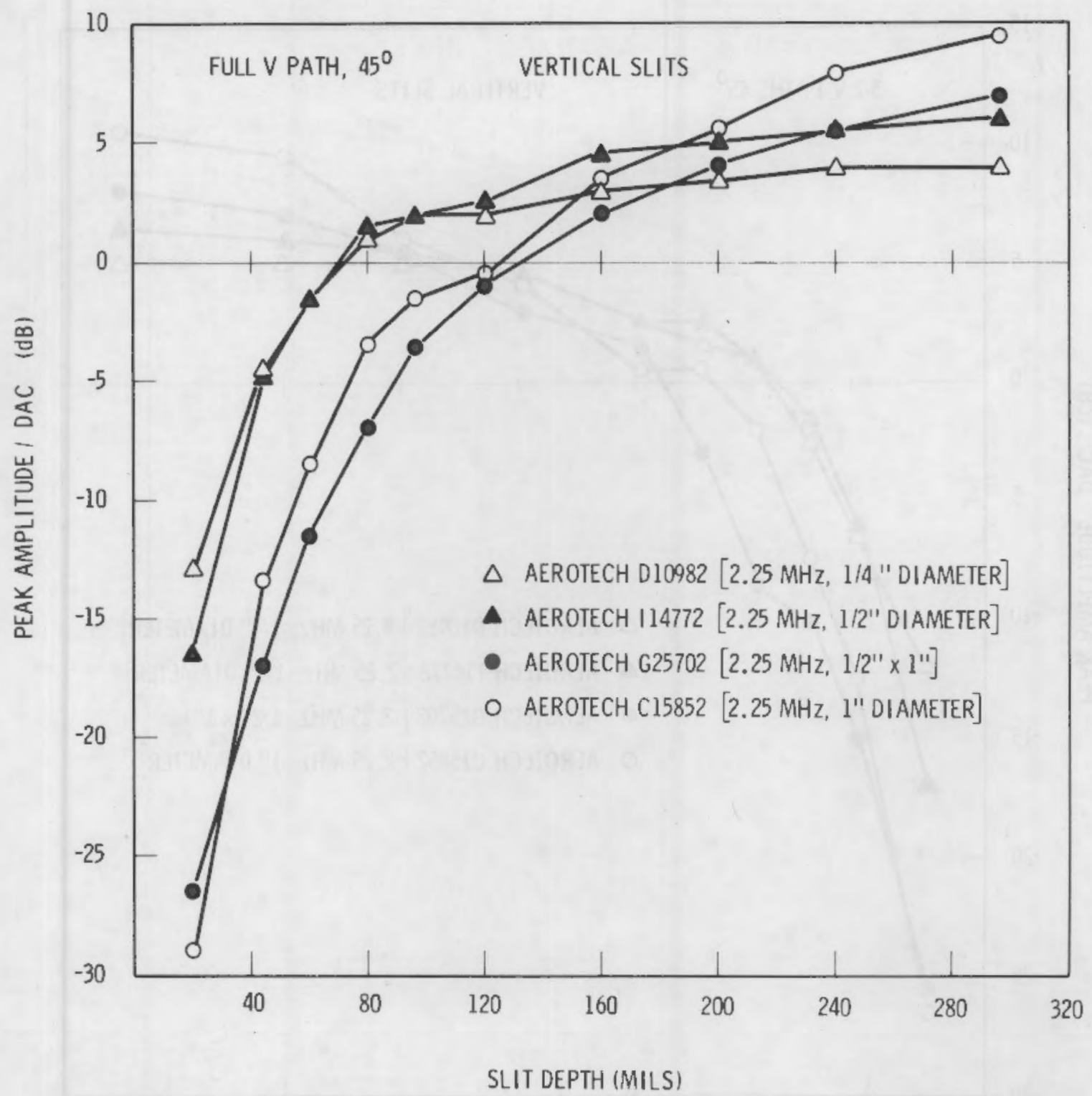

FIGURE 7.2. Ultrasonic Response from Vertical slits, Full V Path, $45^{\circ}$ Beam

3. The $60^{\circ}$ test results indicate that, in general, this angle is less severely influenced by geometrical effects. This results primarily from differences in reflection factors as a function of angle (see Figure 7.11). 


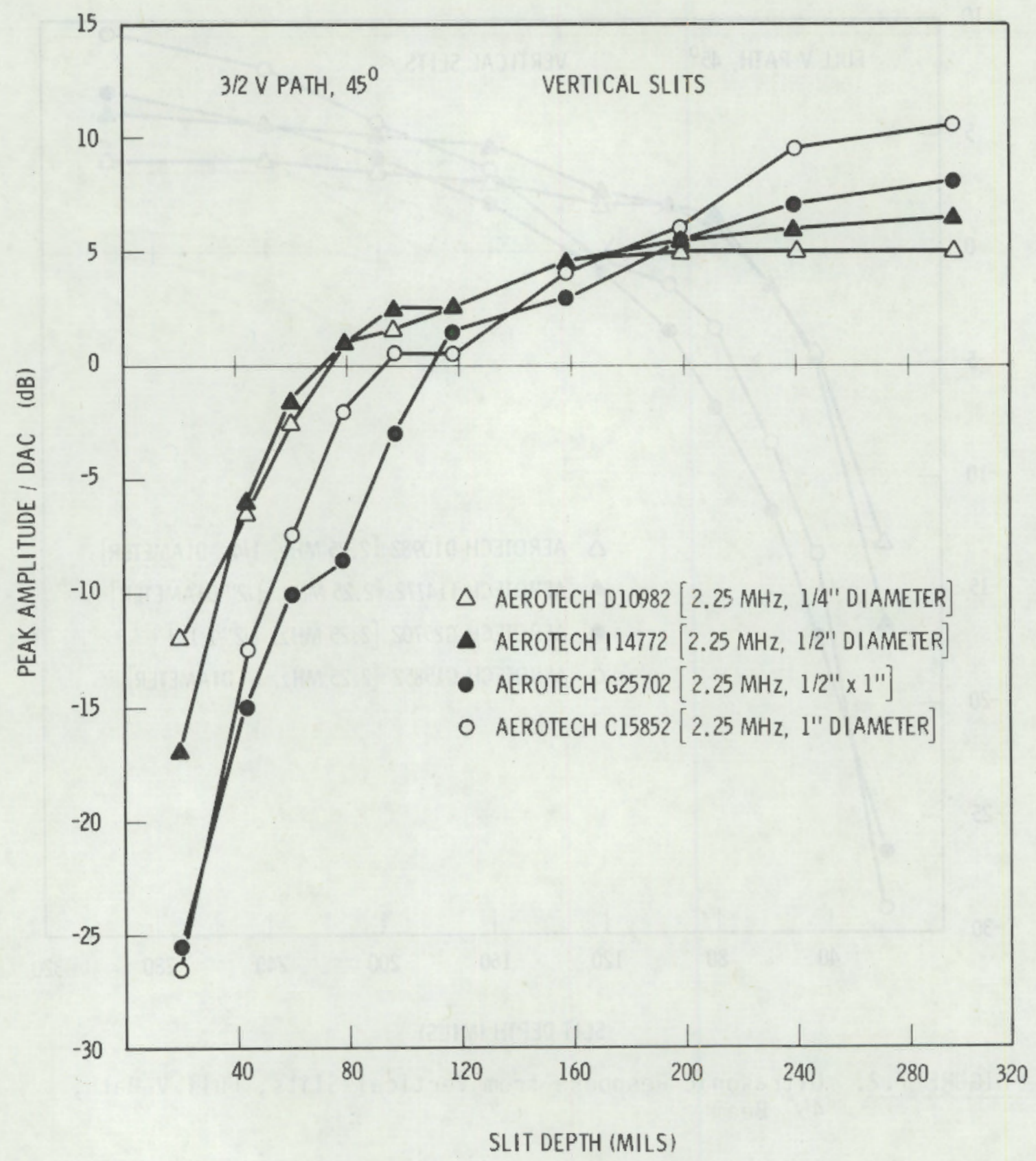

FIGURE 7.3. U1trasonic Response from Vertical Slits, $3 / 2 \mathrm{~V}$ Path, $45^{\circ}$ Beam

4. Figure 7.9 (angled back surface $45^{\circ}$ ) indicates a sharp mininum at $-15^{\circ}$. The incident beam at the back surface in this case is $30^{\circ}$. A $30^{\circ}$ incident angle results in a low reflection factor because of longitudinal wave mode conversion (see Figure 7.11). 


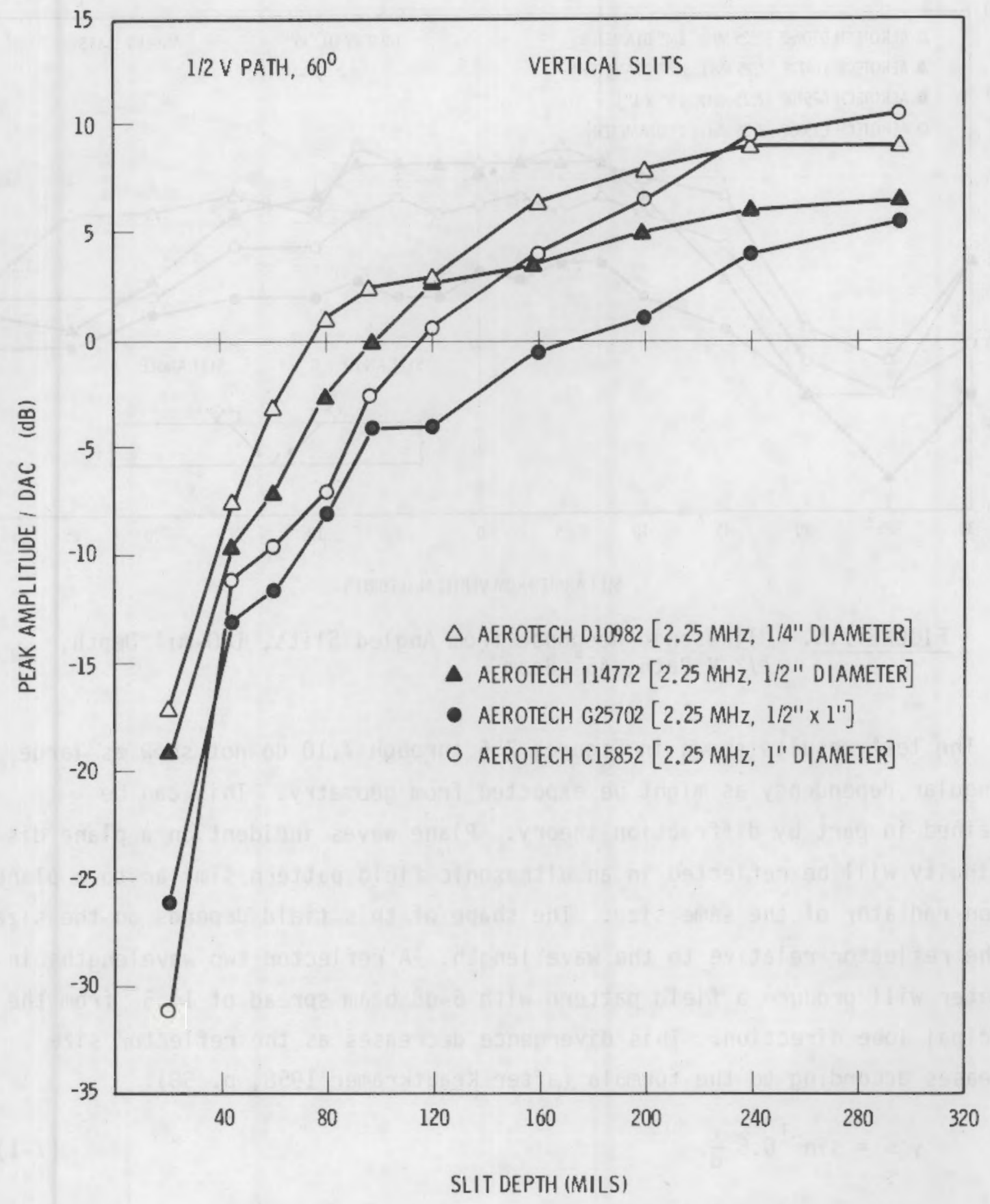

FIGURE 7.4. Ultrasonic Response from Vertical Slits, l/2 V Path, $60^{\circ}$ Beam 


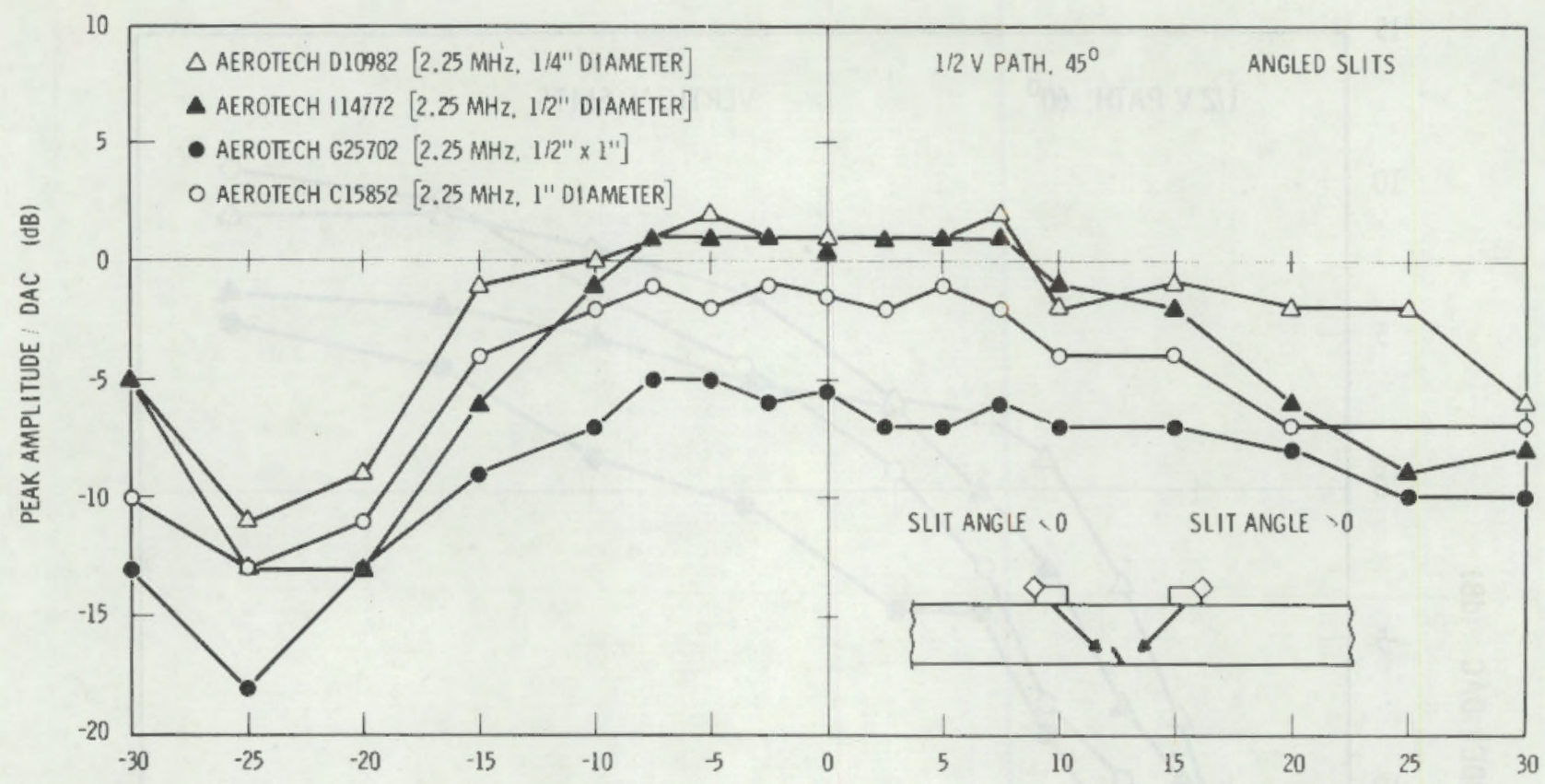

SLIT ANGLE FROM VERTICAL (DEGREES)

FIGURE 7.5. U7trasonic Response from Angled Slits, 100-mil Depth, $1 / 2 \vee$ Path, $45^{\circ}$ Beam

The test results shown in Figures 7.5 through 7.10 do not show as large an angular dependency as might be expected from geometry. This can be explained in part by diffraction theory. Plane waves incident on a plane discontinuity will be reflected in an ultrasonic field pattern similar to a plane piston radiator of the same size. The shape of this field depends on the size of the reflector relative to the wave length. A reflector two wavelengths in diameter will produce a field pattern with 6 - dB beam spread of $14.5^{\circ}$ from the principal lobe direction. This divergence decreases as the reflector size increases according to the formula (after $\mathrm{Krautkramer} \mathrm{1958,0.58).}$

$$
r= \pm \sin ^{-1} 0.5 \frac{\lambda}{d}
$$

where $\lambda$ is the wavelength and $d$ is the diameter of the source (or reflector). The 100-mil notches in these experiments are approximately two wavelengths 


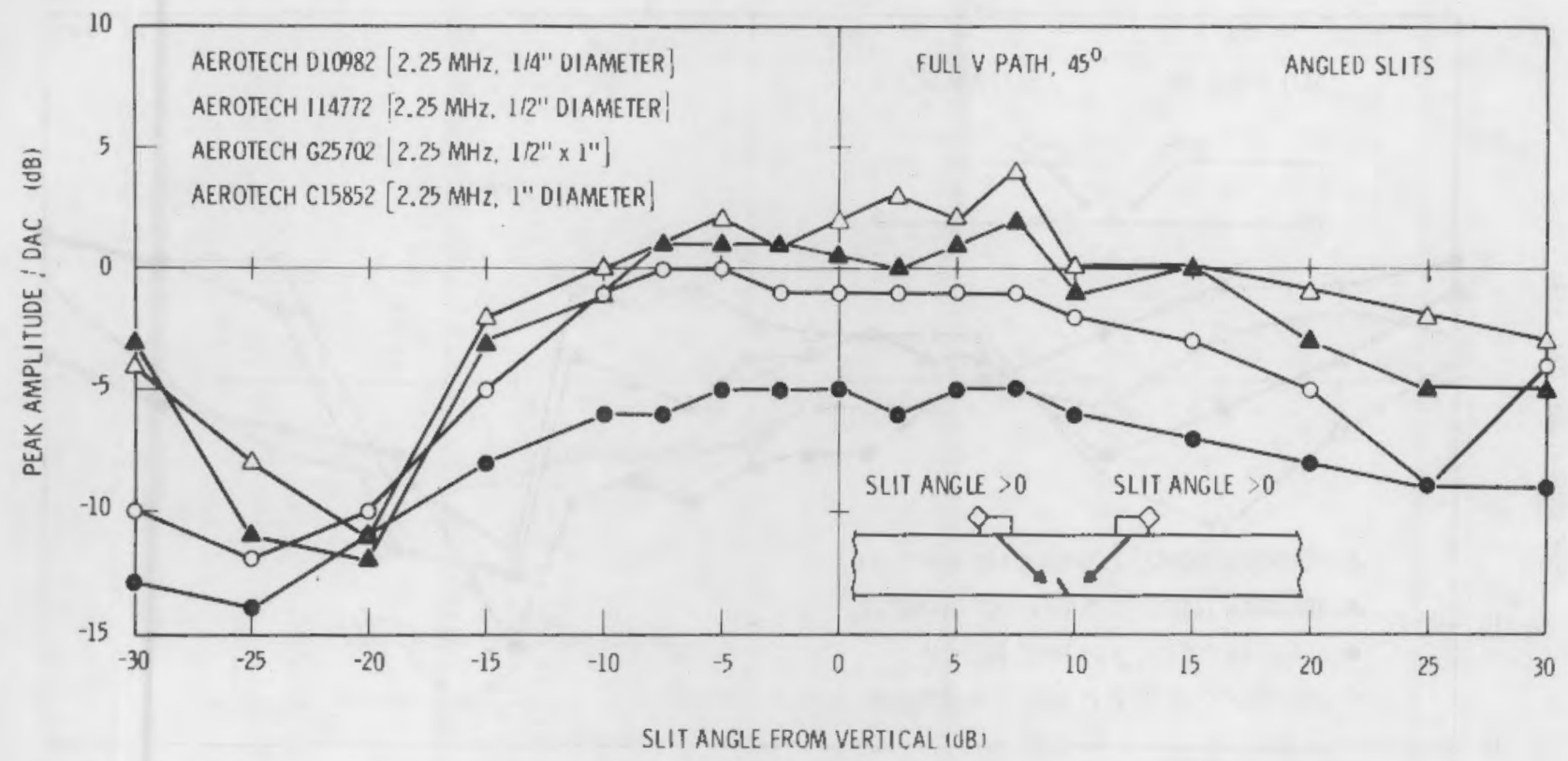

FIGURE 7.5. U1trasonic Response from Angled S1its, 100-mil Depth, Full V Path, $45^{\circ}$ Beam

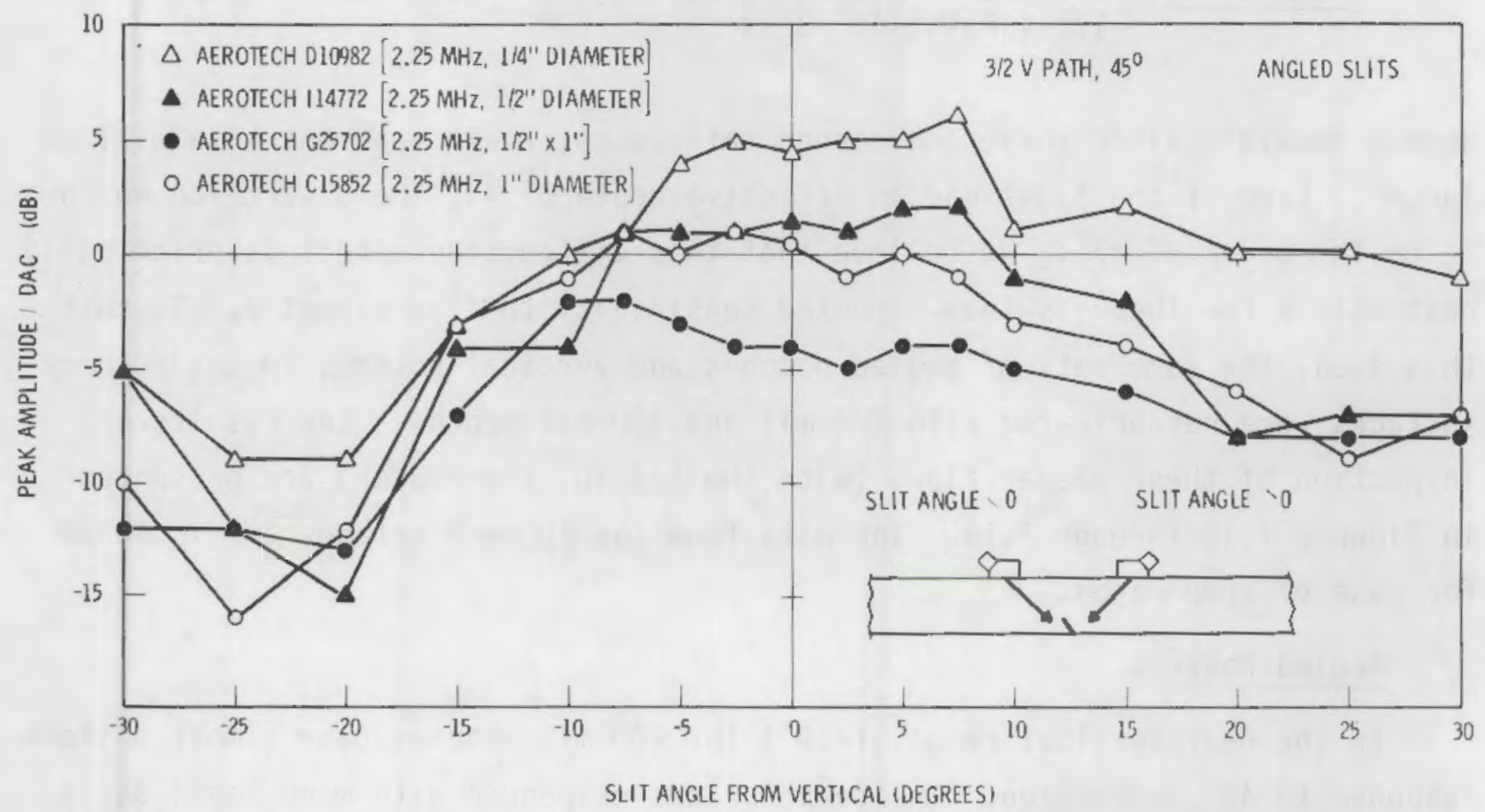

FIGURE 7.7. Ultrasonic Response from Angled Slits, 100-mil Depth, $3 / 2 \vee$ Path, $45^{\circ}$ Bean 


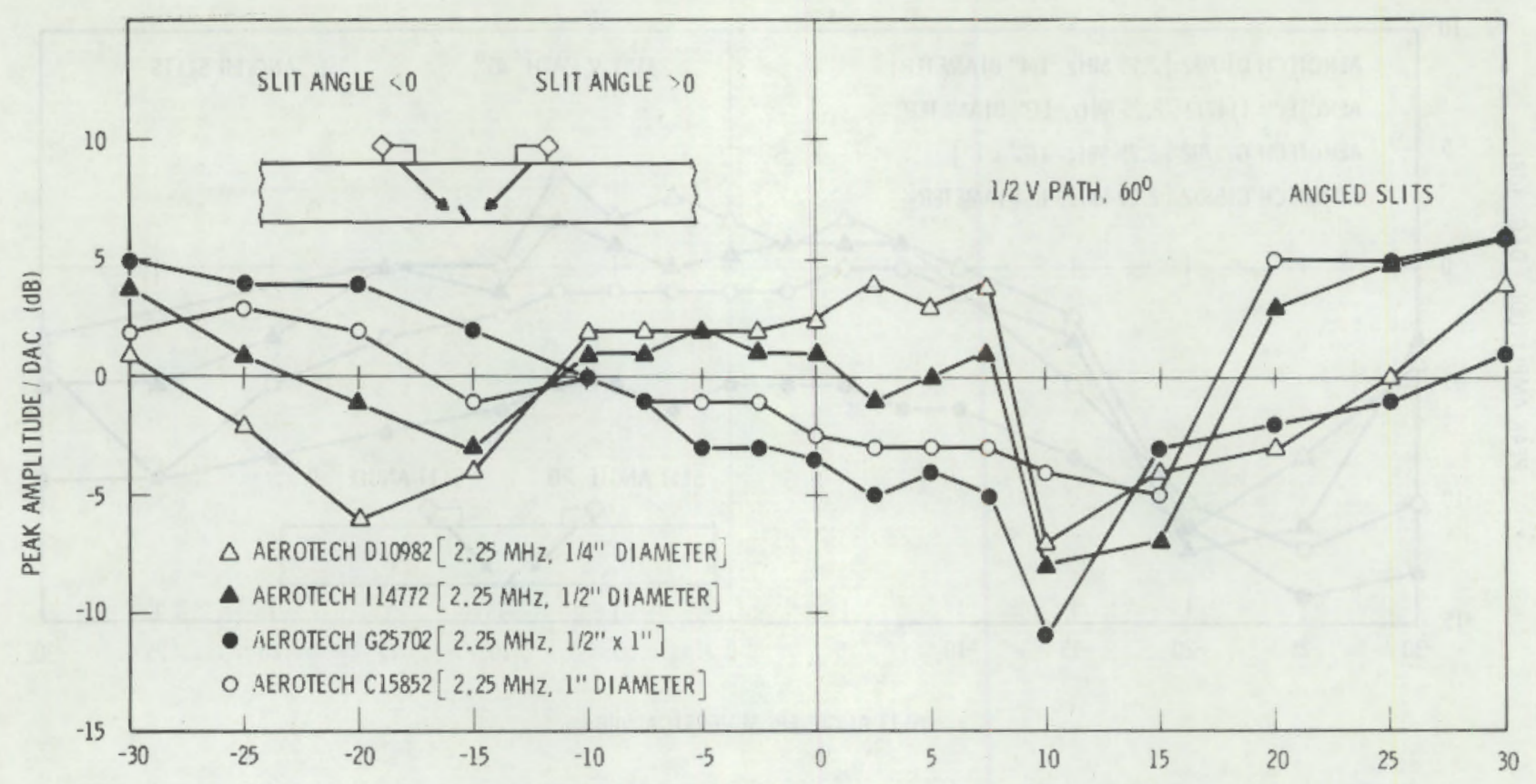

SLIT ANGLE FROM VERTICAL IDEGREES

FIGURE 7.8. Ultrasonic Response from Angled Slits, 100-mil Depth, $1 / 2$ V Path, $60^{\circ}$ Beam

deep. However, since these are corner reflectors, their apparent size will be larger. Even if the flaws had an effective depth of $4 \lambda$, the divergence would be on the order of $\pm 7^{\circ}$. We believe that this diffraction effect is principally responsible for the less-than-expected sensitivity to flaw geometry. To test this idea, the same sets of angled notches and vertical notches in angled back surfaces were refabricated with 200-mil and 300-mil depths. The results of inspection of these deeper flaws (with the 1/2-in. transducer) are presented in Figures 7.12 through 7.18. The data from the 100-mil notches are included for ease of comparison.

\section{Angled Notches}

In the near-vertical range, $\left( \pm 10^{\circ}\right)$ the $100-m i l$ notches gave almost uniform response to $45^{\circ}$ inspection. The deeper flaws responded with more small-angle sensitivity; the response dropped significantly for flaw angles of $5^{\circ}$ or more from vertical. In the range $-2.5^{\circ}$ to $+2.5^{\circ}$, signal amplitude increased with flaw depth. 


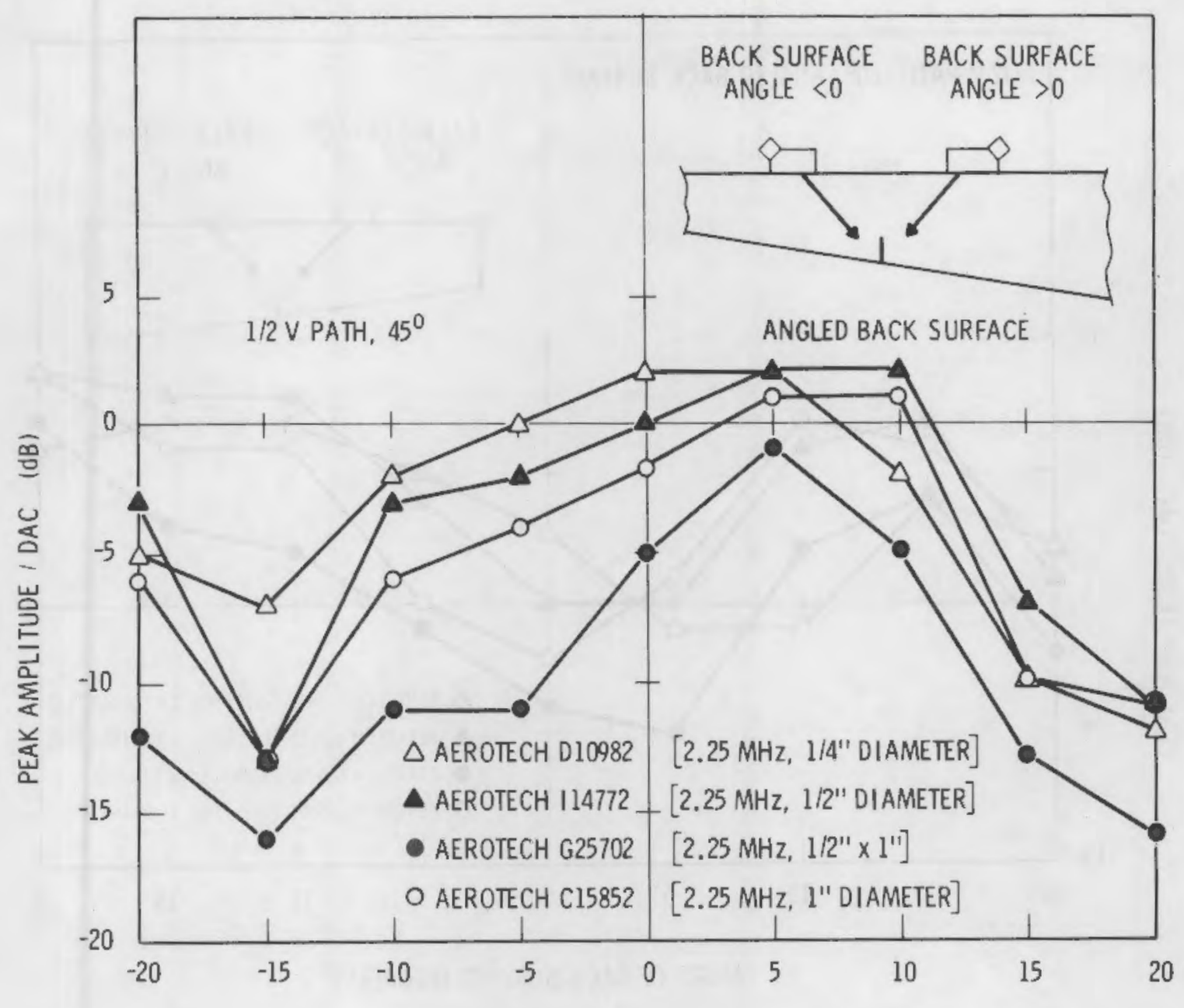

ANGLE OF BACK SURFACE (DEGREES)

FIGURE 7.9. Ultrasonic Response from Vertical, 100-mil S1its in Angled Back Surfaces, $1 / 2 \vee$ Path, $45^{\circ}$ Beam

The $+15^{\circ}$ and $-15^{\circ}$ slits were expected to yield minimal response in a $45^{\circ}$ inspection because each involves a $30^{\circ}$ (from normal) incidence angle, and consequently mode conversion results in a large energy loss. The deeper flaws did show a sharp drop in response at these angles, while the 100-mil flaws did not.

From $+20^{\circ}$ to $+30^{\circ}$, all three slit depths produce approximately the same response amplitude for $45^{\circ}$ inspection, about 5 to 8 dB below DAC. This indicates that with a 50\% DAC recording criterion, flaws at these angles and with through-wall depths up to $50 \%$ will be at best marginally detectable. 


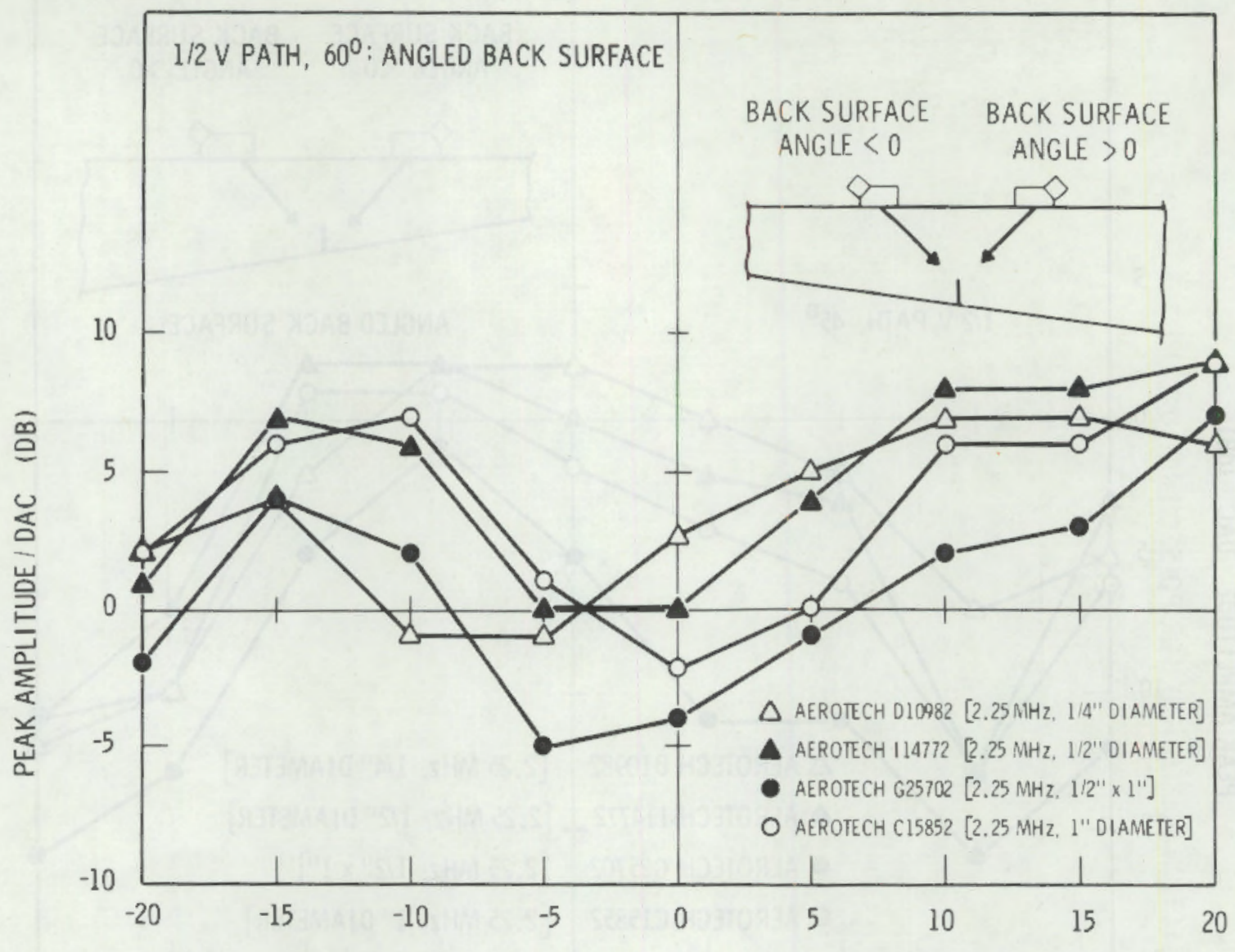

ANGLE OF BACK SURFACE (DEGREES)

FIGURE 7.10. Ultrasonic Response from Vertical, 100-mil Slits in Angled Back Surfaces, $1 / 2$ V Path, $60^{\circ}$ Beam

The deeper slits at $-20^{\circ}$ to $-30^{\circ}$ angles give higher responses to $45^{\circ}$ inspection; about two or three $\mathrm{dB}$ below $\mathrm{DAC}$ for $1 / 2 \mathrm{~V}$ patn length, and about equal to DAC for full $V$ and $3 / 2 \mathrm{~V}$ paths. The 100 -mil slits behave very differently; while the $-30^{\circ}$ response is nearly as high as that of the 200mil and $300-m i l$ slits, the $-20^{\circ}$ and $-25^{\circ}$ responses are quite low, falling between 10 and $15 \mathrm{~dB}$ below DAC. We do not know why; their smaller size is not enough to account for such a marked difference in response amplitude.

For $60^{\circ}$ inspection, the only slit angles that are troublesome from the standpoint of a 50\% DAC recording criterion are $+10^{\circ}$ and $+15^{\circ}$. Responses are near or above DAC (for all depths) for the slit angle ranges $-30^{\circ}$ to $-20^{\circ},-10^{\circ}$ to $+2.5^{\circ}$, and $+20^{\circ}$ to $+30^{\circ}$. The response drops rapidly for small positive slit 


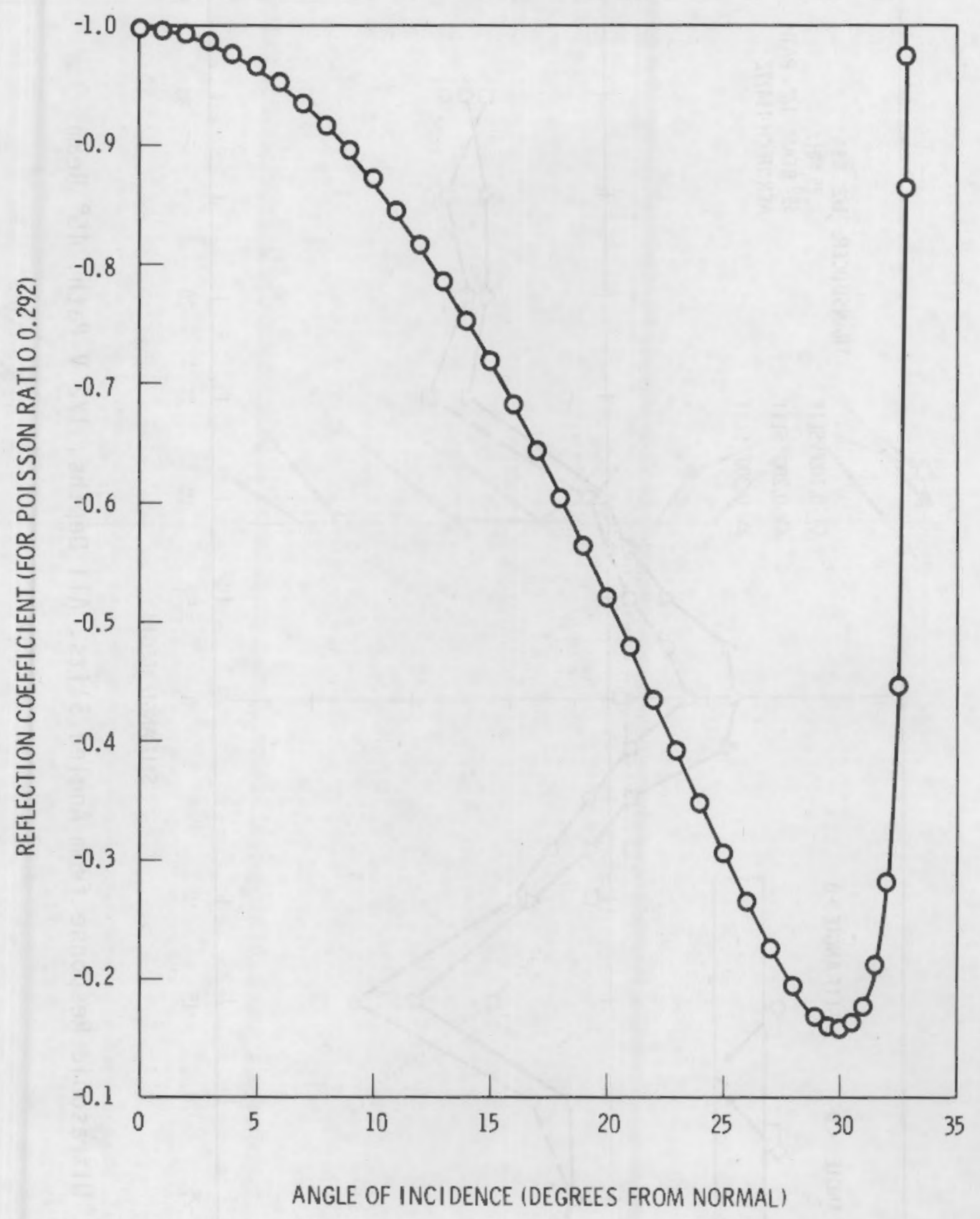

\section{FIGURE 7.11. Reflection Coefficient for Shear Wave Incident Upon Steel-to-Air Interface, (After Krautkramer and Krautkramer 1969, p. 480)}

angles because the corresponding reflection angles have low reflection coefficients. However, the angled flaws likely to be encountered in the field (lack 


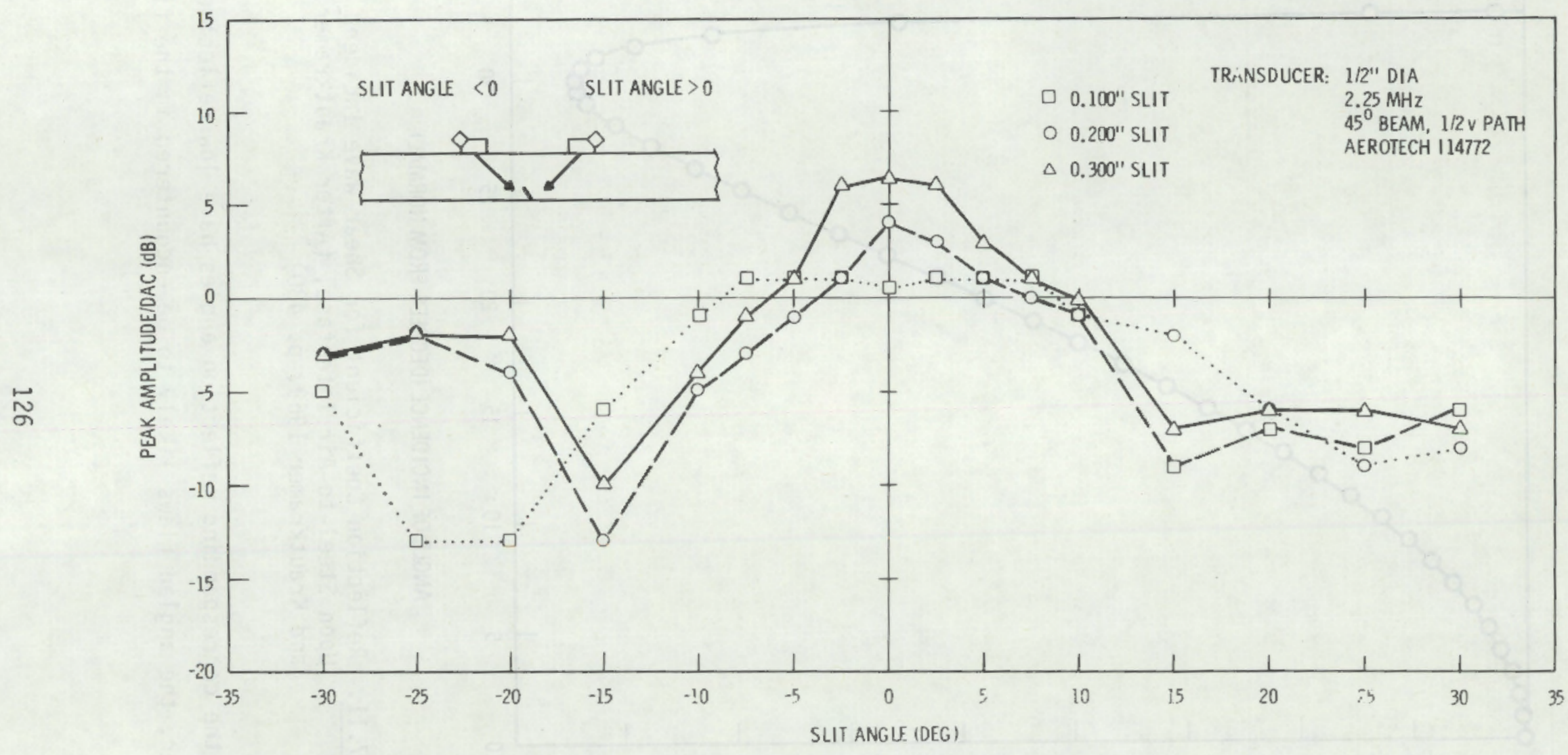

FIGURE 7.12. UTtrasonic Response from Angled S1its, All Depths, $1 / 2 \mathrm{~V}$ Path, $45^{\circ}$ Beam 


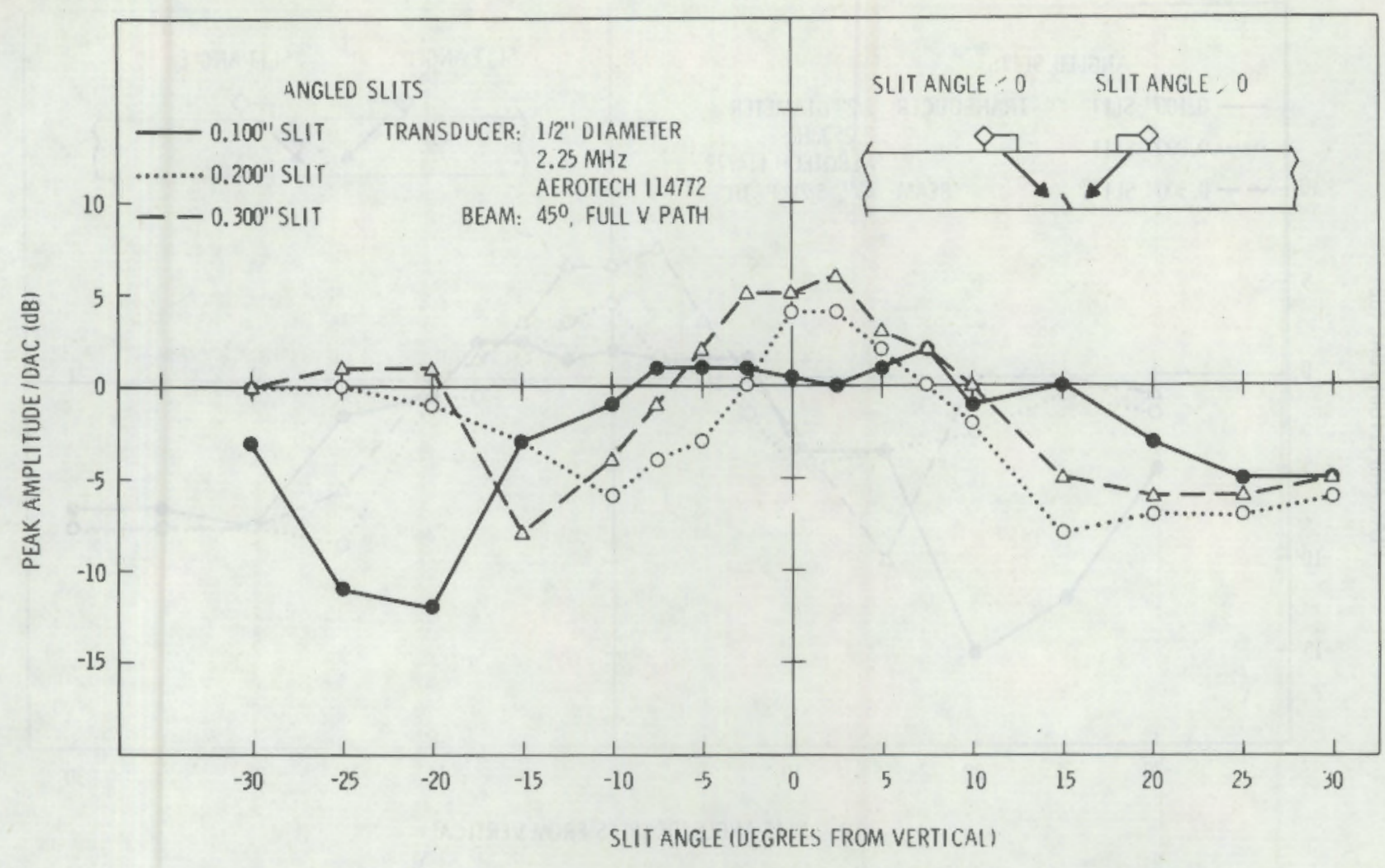

FIGURE 7.13. Ultrasonic Response from Angled Slits, All Depths, Full V Path, $45^{\circ}$ Beam

of fusion, for example) would be inspected from the negative direction and the only negative angle that might give problems is $-15^{\circ}$. The responses at $-15^{\circ}$ were 3 to $5 \mathrm{~dB}$ below DAC.

\section{Vertical Slits in Angled Back Surfaces}

The responses of the deep slits do not vary markedly from that of the 100mil slits in $45^{\circ}$ inspection. All depths show a sharp minimum at $-10^{\circ}$ to $-15^{\circ}$ (mode conversion). Positive angles are most likely to be encountered in field inspection; responses from inspection of flaws on $+5^{\circ}$ and $+10^{\circ}$ counterbores are above DAC, while $+15^{\circ}$ and $+20^{\circ}$ responses are at or below 50\% DAC. ASME Boiler and Pressure Vessel Code, Section III specifies counterbore angles of less than $15^{\circ}$. The data indicates that under high angle counterbore conditions, vertical flaws will be only marginally detectable (for all depths) at the 50\% DAC level when using $45^{\circ}$ inspection. 


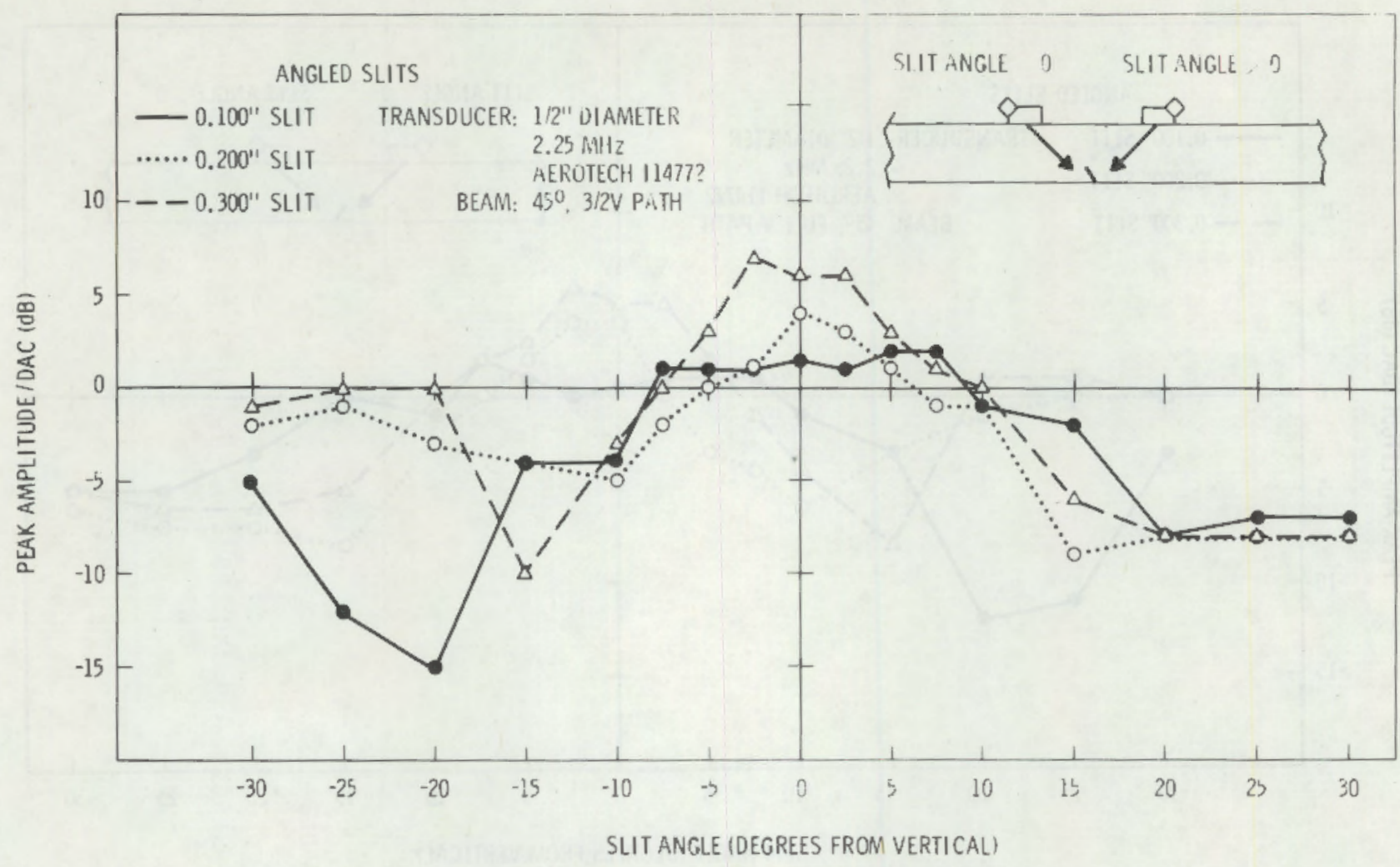

FIGURE 7.14. Ultrasonic Response from Angled Slits, All Depths, $3 / 2$ V Path, $45^{\circ}$ Beam

Inspection with a $60^{\circ}$ beam produced much higher amplitude; nearly every response was at or above DAC. No investigated condition of slit depth and counterbore angle presents detectability problems.

Notch Detection in Simulated Thicker-Wall Pipe

The data can be used to evaluate detectability for inspection of flaws in thicker pipe. For our flat 0.6-in. plates, a full $\mathrm{V}$ inspection of a flaw is identical to a $1 / 2 \mathrm{~V}$ inspection of the same flaw in 1.2-in. material. Likewise, $3 / 2 \vee$ inspection of 0.6 -in. plate corresponds to $1 / 2 \vee$ inspection in 1.8-in. plate. ASME calibration notches with 0.120-in. and $0.180-i n$. depths are used to calibrate these inspections. The results are presented in Figures 7.18 through 7.20 , with reflection amplitude plotted against flaw depth in percent of wall thickness for all three thicknesses with three trandsucer diameters. The influence of wall thickness on detectability is best illustrated by Figure 7.20 , for the 1-in. transducer. 


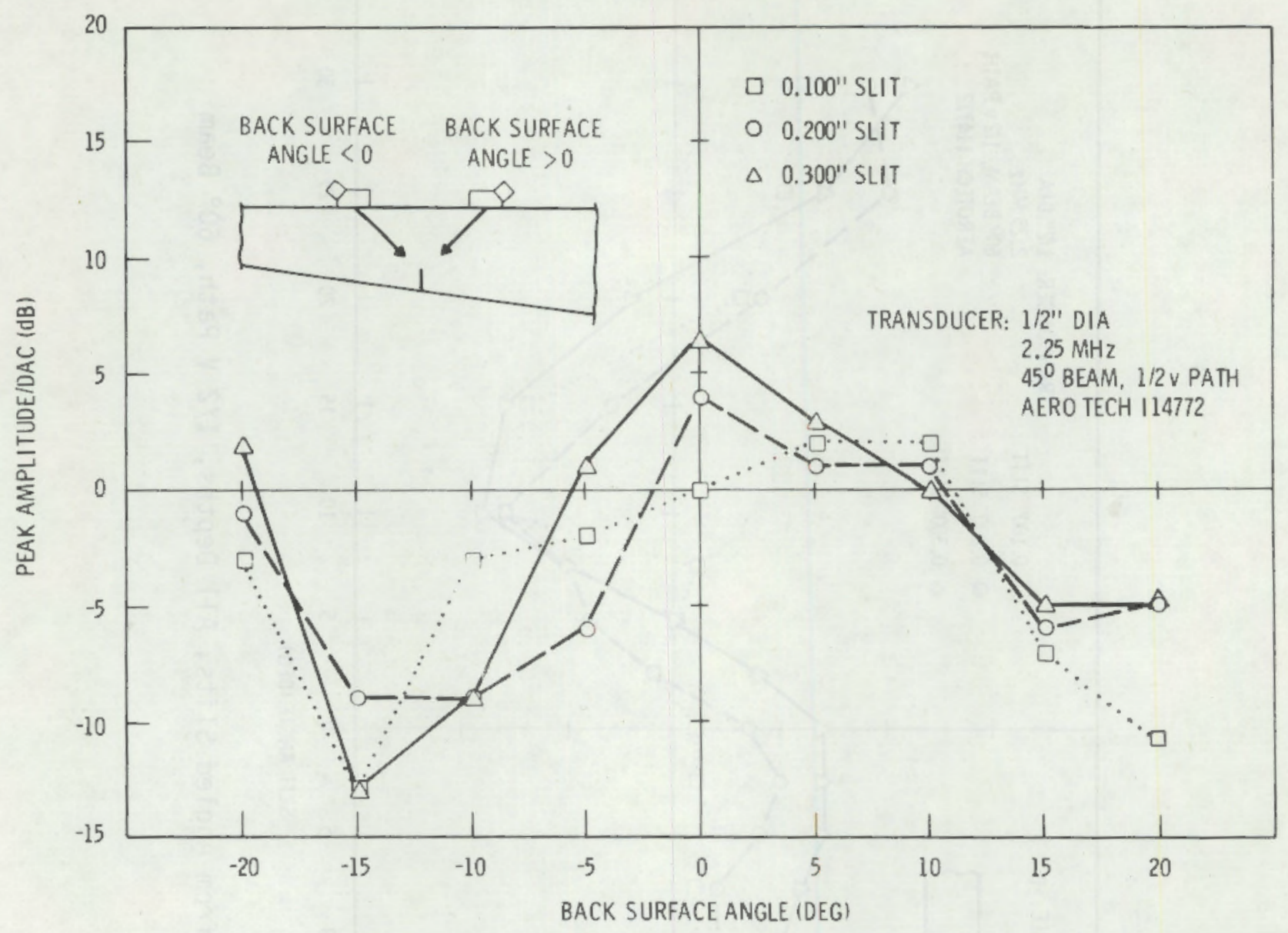

FIGURE 7.16. Ultrasonic Response from Vertical Slits in Angled Back Surfaces, All Depths, $1 / 2 \vee$ Path, $45^{\circ}$ Beam

According to Table IWB-3514.3 of ASME Section XI Code, the maximum allowable flaw depth for this aspect ratio is about $11 \%$ through wall. Figure 7.20 shows clearly that for $11 \%$ crack depth, the reflection amplitude is powerfully influenced by wall thickness; small thickness results in sinall response. In fact, the maximum allowable flaw in 0.6-in. pipe is seen to be undetectable with a $50 \%$ DAC recording criterion.

This effect is not as great for the 1/4-in. and 1/2-in. transducers (Figures 7.18 and 7.19 ) because the $11 \%$ flaws are large enough to be near the infinite-reflector response plateau and, therefore, have about the same response. 


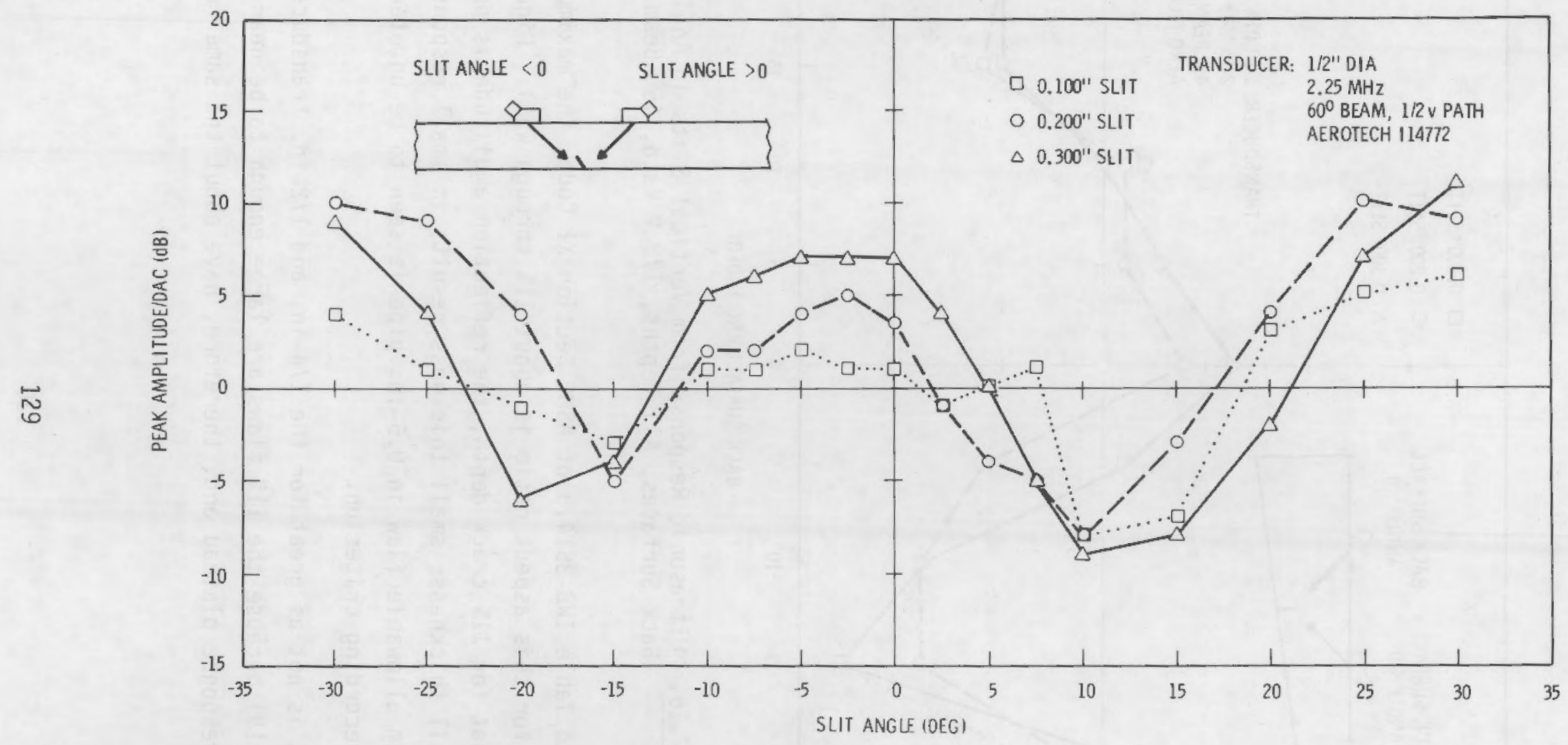

FIGURE 7.15. Ultrasonic Response from Angled S1its, All Depths, 1/2 V Path, $60^{\circ}$ Beam 


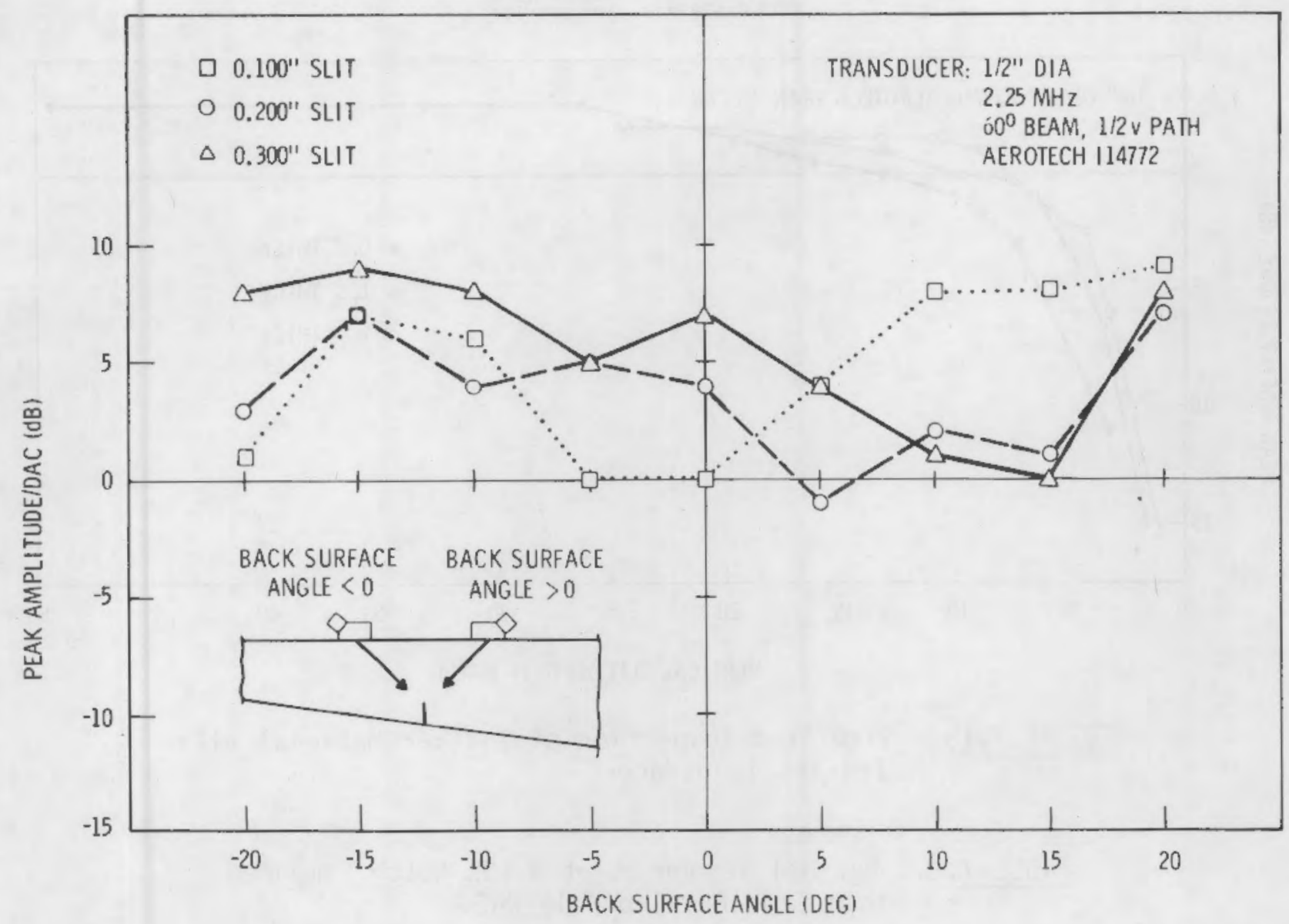

FIGURE 7.17. Ultrasonic Response from Vertical Slits in Angled Back Surfaces, All Depths, $1 / 2 \mathrm{~V}$ Path, $60^{\circ}$ Beam

\section{Comparison of Code Calibration Reflectors}

The basic calibration reflector for these studies is the end-mill notch with a depth equal to $10 \%$ of the pipe wall thickness. Another calioration reflector often used is the side-drilled hole required by the 1974 version of ASME Section XI Code. If the two calibrations differ significantly, they will not produce equal levels of detection reliability.

In order to compare the two, calibration was performed using the sidedrilled hole, and then the $10 \%$ notch was inspected. This was done for $45^{\circ}$ beam $(1 / 2 \mathrm{~V}$, full $\mathrm{V}, 3 / 2 \mathrm{~V})$ and $60^{\circ}$ beam $(1 / 2 \mathrm{~V})$ and for several transducers. The results are shown in Table 7.1 . 


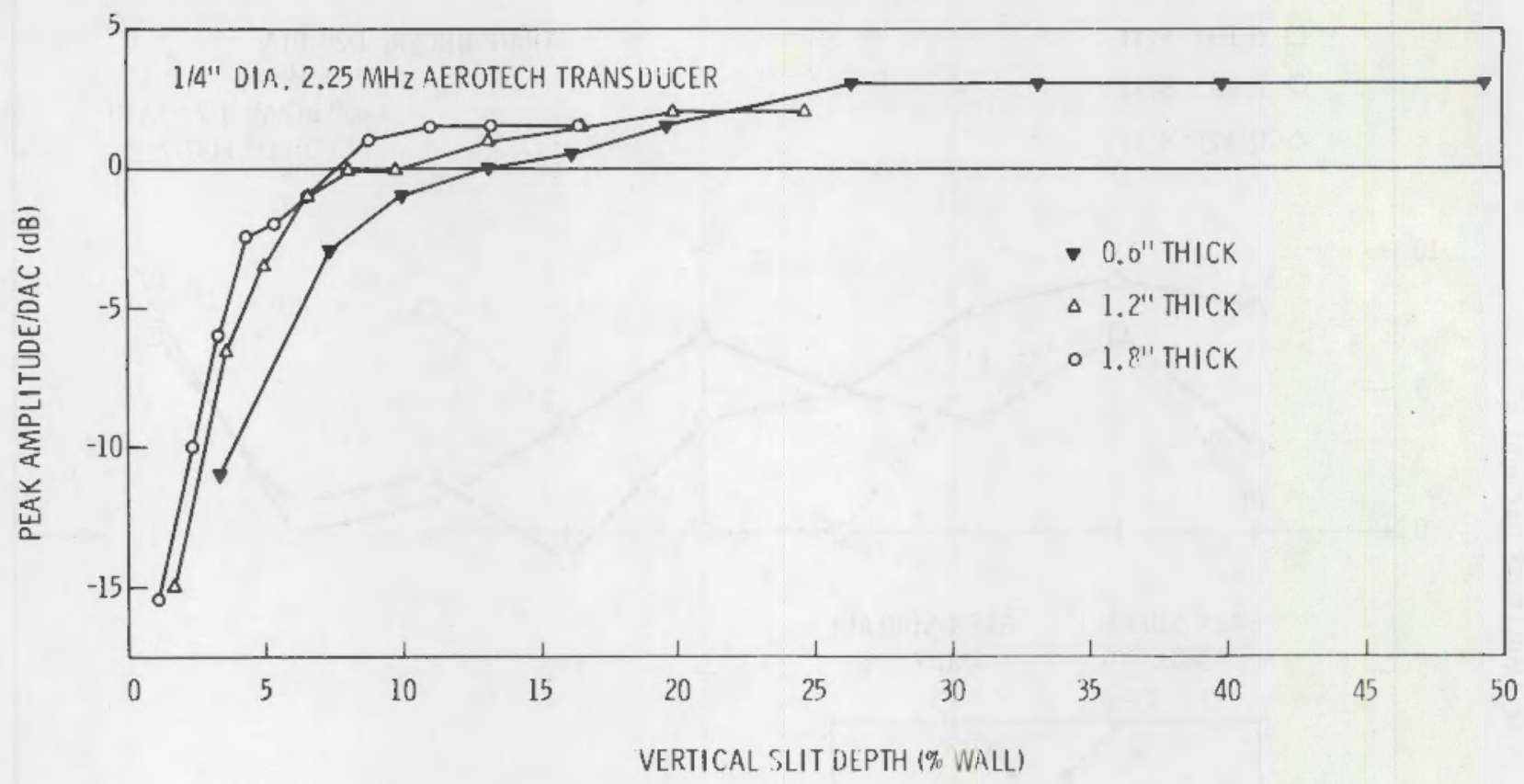

FIGURE 7.18. Simulated Inspection of Thicker Material with $1 / 4$ in. Transducer

TABLE 7.1. Decibel Responses of a $10 \%$ Notch Compared to a side-Orilled Hole OAC

\begin{tabular}{|c|c|c|c|c|}
\hline $\begin{array}{c}\text { Transducer } \\
\text { Specifications }\end{array}$ & $\begin{array}{l}10 \% \text { Notch } \\
1 / 2 \vee 45^{\circ}\end{array}$ & $\begin{array}{l}\text { Response, } \mathrm{dB} \\
\text { Ful1 } \vee 45^{\circ}\end{array}$ & $\begin{array}{r}\text { Site-or } \\
\text { Res } \\
3 / 2 \mathrm{~V}-45 \\
\end{array}$ & $\begin{array}{l}\text { Hole } \\
\frac{d B}{12 V 60^{\circ}}\end{array}$ \\
\hline $1 / 4$ in., $2.25 \mathrm{MHz}$ & +9 & +11 & +16 & +2 \\
\hline $1 / 2$ in., $2.25 \mathrm{MHz}$ & +9 & +3 & +3 & 0 \\
\hline 1 in., $2.25 \mathrm{MHz}$ & +3 & +3 & +2 & -3 \\
\hline
\end{tabular}

In the $45^{\circ}$ inspection, the $10 \%$ notch produced higher responses than the side-drilled hole for all beam paths and transducer diameters. Since a higher calibration response means reduced sensitivity, the $10 \%$ notch calibration results in a less sensitive inspection. The hole response is lower because the reflection area is smaller. 


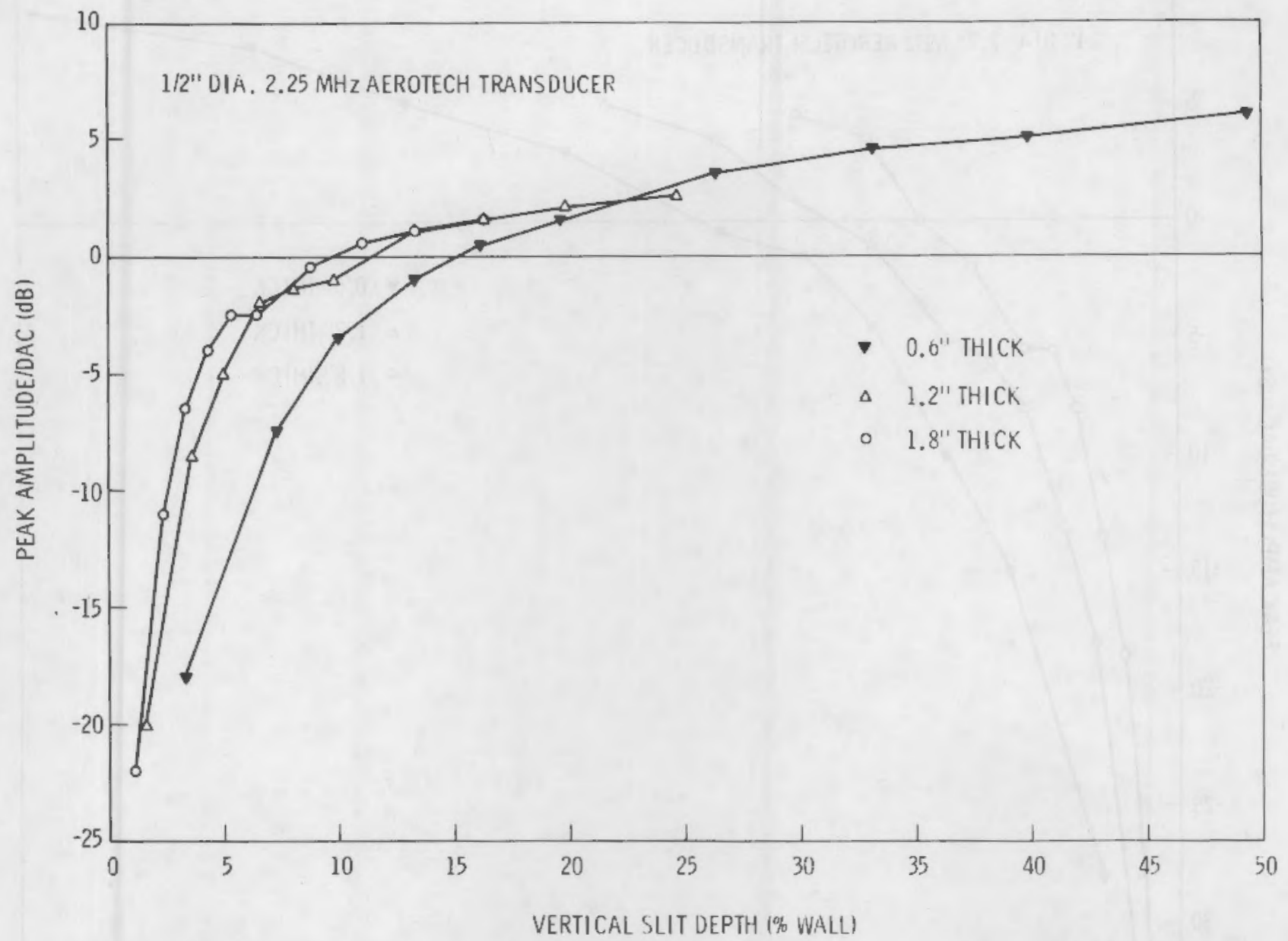

FIGURE 7.19. Simulated Inspection of Thicker Material with $1 / 2-i n$. Transducer

In calibrating for a $60^{\circ}$ inspection, the larger area of the notch is negated by mode conversion and the sensitivities of the two calibrations are about equal. No mode conversion occurs upon reflection from the hole because all reflections from a side-drilled hole are at normal incidence.

Summary: Impact of Flaw Geometry Results on Inspection Reliability

Test results indicate that several factors will influence ISI raliability. One is search unit selection. Even though calibration is performed strictly to code (Section XI, 10\% notch), test results couid be highly variable (5 dB or more) without requirements for search unit size and operating characteristics. Also, choosing a search unit requires a compromise between small flaw 


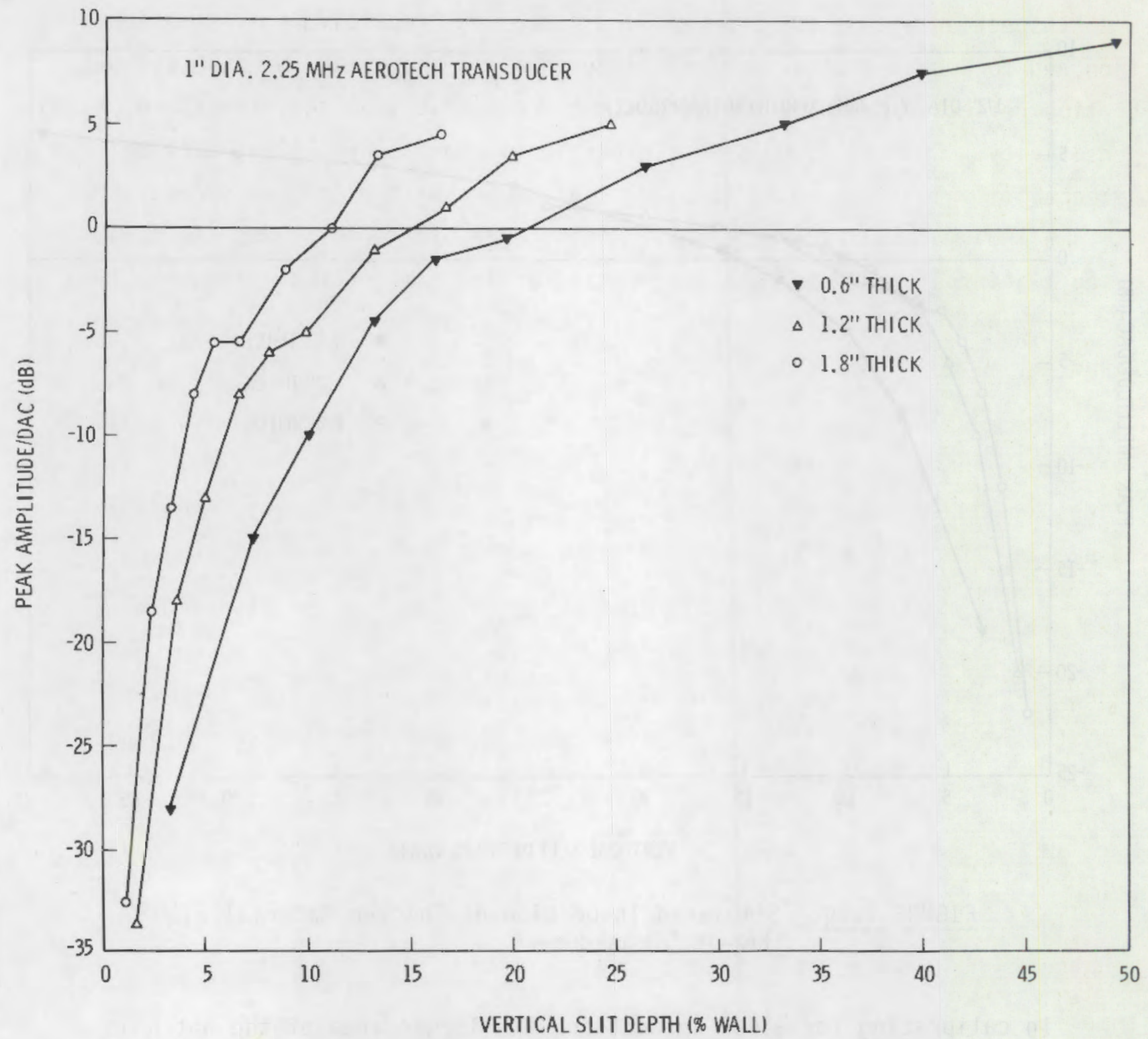

FIGURE 7.20. Simulated Inspection of Thicker Material with 1-in. Transducer

and off-angle flaw detectability on one hand and large flaw detectability on the other.

Ultrasonic response for angled flaws and flaws on the counterbore varies widely with angle and flaw depth. In some cases, a small flaw can produce a higher response than a large flaw of the same orientation. It is entirely possible that as certain cracks grow, periodic inspections would show decreasing signal amplitude. 
Inspection with a $60^{\circ}$ beam appears to be less sensitive to flaw orientation and to provide better detectability than $45^{\circ}$ inspection because the calibration reflector produces almost maximum mode conversion and, therefore, high sensitivity. Currently, only $45^{\circ}$ inspection is mandatory. Also, off-angle detectability is improved by choosing transducers with high beam spread, that is, small diameter or low frequency. This can apply to instrument selection-given two inspection instruments operating at the same nominal frequency, the one with the lower actual frequency will provide better angled flaw and counterbore flaw detectability.

Choice of calibration reflector can be highly influential for $45^{\circ}$. inspection; for transducers smaller than 1 in., the use of side-drilled holes will lead to a much more sensitive inspection. For large transducers, and for a $60^{\circ}$ inspection, there is not so much difference.

Finally, the detectability of the maximum allowable flaw witi a given transducer varies with wall thickness. The variation is not large for small transducers but is large for the 1-in. transducer; detectability increased dramatically as thickness increased. Use of the 1-in. transducer would not provide a good inspection for this 10 -in., Schedule 80 pipe.

\subsection{EFFECTS OF FLAW CHARACTERISTICS ON INSPECTION RELIABILITY}

The objective of these measurements is to define the influence of crack roughness and crack tightness on detection reliability.

Samples

Two types of flaws were produced for study: mechanical fatigue cracks produced by four-point bending and thermal fatigue cracks. In all, 38 samples were fabricated from 304 stainless steel. Twenty-six of these contain one bending fatigue crack each and 12 contain one thermal fatigue crack each.

The bending fatigue samples are $4 \mathrm{in}$. wide and $3 / 4 \mathrm{in}$. thick, cold-rolled down from a l-in. thickness. After the cracks were grown, the starter notches were machined off leaving the bars' thicknesses at slightly over 0.5 in. in the neighborhood of the cracks. 
In order to produce aspect ratios of approximately 4 and 10 , drilled holes and saw-cut notches were (respectively) used as crack initiators. The approach proved unsuccessful as the aspect ratios obtained ranged mainly between 4 and 6, with no apparent correlation between aspect ratio achieved and type of initiator used.

Crack roughness refers to the load and cycle rate used to grow the cracks. The cracks herein referred to as "rough" were loaded with $50.4 \mathrm{Ksi}$ maximum stress change at a rate of $4 \mathrm{~Hz}$. The "smooth" cracks were loaded with $28.8 \mathrm{Ksi}$ maximum stress change at a rate of $7 \mathrm{~Hz}$. Crack growth rates were on the order of $10^{-6}$ in./cycle for the smooth flaws, and $10^{-5}$ to $10^{-4}$ in./cycle for the rough flaws.

The range of depths of the twelve bending fatigue cracks that were examined destructively was 68-235 mils. The crack faces were all quite vertical and most were very flat, though a few had step discontinuities. Most of these discontinuities were significantly large compared to the wavelength (greater than $1 / 4$ wavelength). The phase interference resulting from these discontinuities caused lower maximum signal amplitudes and the position of maximum amplitude was displaced from the center of the crack.

In depth, aspect ratio and orientation, these bending fatigue cracks are similar to the normal vertical EOM flaws mentioned earlier.

of the twelve 4-in.-wide thermal fatigue samples, six are $3 / 4-i n$. thick (cold-rolled from l-in. thickness), and six are 0.6 in. thick. The latter samples were annealed before thermal cycling. After crack growth was completed, the starter notches were machined off, but a minimal amount of material was removed and no attempt was made to reduce the thickness of the $3 / 4$-in. pieces to $0.6 \mathrm{in}$.

Four of the thermal fatigue cracks were examined destructively. These cracks are extremely tight and rough, and their shapes and orientations vary widely. The crack faces show ripples, twists and changes in the direction of propagation.

Two specimens were taken from each of four samples for measurement of yield strength. The results are in Table 7.2 below. Sample numbers beginning 
with " $A$ " refer to bending fatigue samples and sample numbers beginning with "B" are thermal fatigue samples. The annealed thermal fatigue sample B114 was annealed before crack growth. The annealed bending fatigue sample A142 was annealed in preparation for further ultrasonic measurement after crack growth and ultrasonic inspection had been done.

TABLE 7.2. Yield Stress of Cold-Rolled and Annealed Samples

Sample

A140

A140

B103

8103

$\begin{array}{ll}\text { A142 } & \text { Annealed } \\ \text { A142 } & \text { Annealed } \\ \text { B114 } & \text { Annealed } \\ \text { B114 } & \text { Annealed }\end{array}$

Yield Stress, ksi

$\left.\begin{array}{c}95.4 \\ 114.0 \\ 102.4 \\ 90.15\end{array}\right) \quad$ Mean:

$$
\begin{aligned}
& 49.9 \text { 49.1 Mean: } \\
& 47.4 \quad 47.8 \\
& 44.6
\end{aligned}
$$

\section{Flaw Measurement Results - Bending Fatigue Samples}

\section{Crack Depth Sizing}

Methods investigated for nondestructive determination of crack depth were: satellite pulse technique (Gruber 1979), electrical resistance gauge, probe motion, and echo amplitude.

The satellite pulse technique (SPT) determines crack depth by measurement of the difference in time-of-flight between the shear wave reflected from the crack and the shear wave diffracted from the crack tip (Figure 7.21). Sometimes this technique works well but more often, the diffracted signal (satellite pulse) is buried in noise and can be distinguished only with difficulty, if at all. Also in some cases, another signal can be mistaken for the satellite, resulting in an erroneous depth determination. This method is insufficiently reliable. 


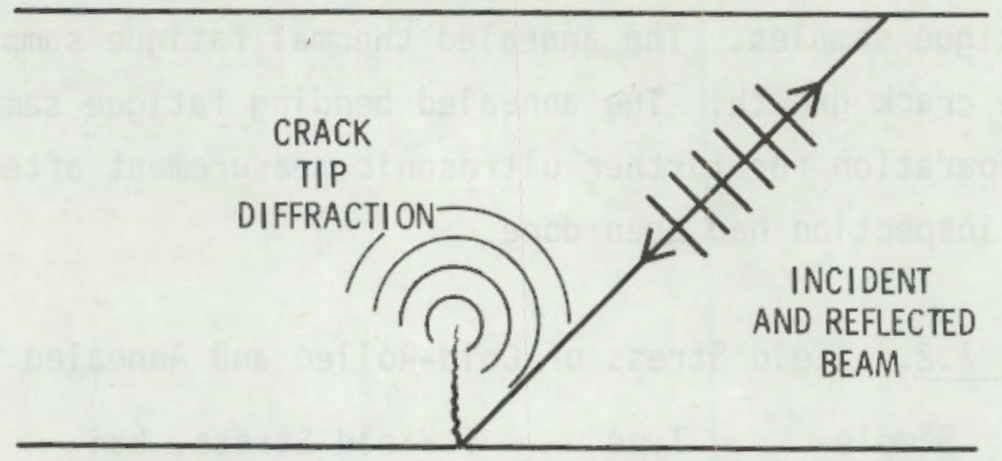

FIGURE 7.21. Satellite Pulse Technique for Crack Sizing

The electrical resistance gauge (ERG), a commercial instrument manufactured by Test Systems International, Inc., measures the electrical resistance resulting from current flow around the crack and can be calibrated using EDM notches as a standard. The major limitations are conduction around the edges of low-aspect-ratio cracks and conduction through very tight cracks, both of which would cause a low depth reading. These are apparently no problem for the bending fatigue cracks, as this was the best of the sizing methods investigated. The correlation between ERG depth determinations and destructive depth measurements is illustrated graphically in Figure 7.22. For inservice sizing of reactor piping cracks, the ERG could only be used for cracks opening on the outer diameter of the pipe. This is because use of the ERG involves pressing a probe against the cracked surface.

Probe motion involves moving a transducer toward a crack and observing the reflection as it grows and then fades. As an example, one might measure the change in transducer position between the two points which give a signal $6 \mathrm{~dB}$ below maximum. An empirical relation can be developed between probe motion and crack depth. Twelve probe motion measurements were made, that is, with four different measuring points $(-6 \mathrm{~dB}$ points, $-20 \mathrm{~dB}$ points, vanishing points, and two points a fixed voltage from baseline) and three transducer setups (3/4-in., 2.5-MHz unfocused, 1/2-in., 5-MHz focused, and a shadow technique with the former sending and the latter receiving) for each crack. Then each of the twelve resulting data pair sets was given a least-squares fit to linear, exponential, 


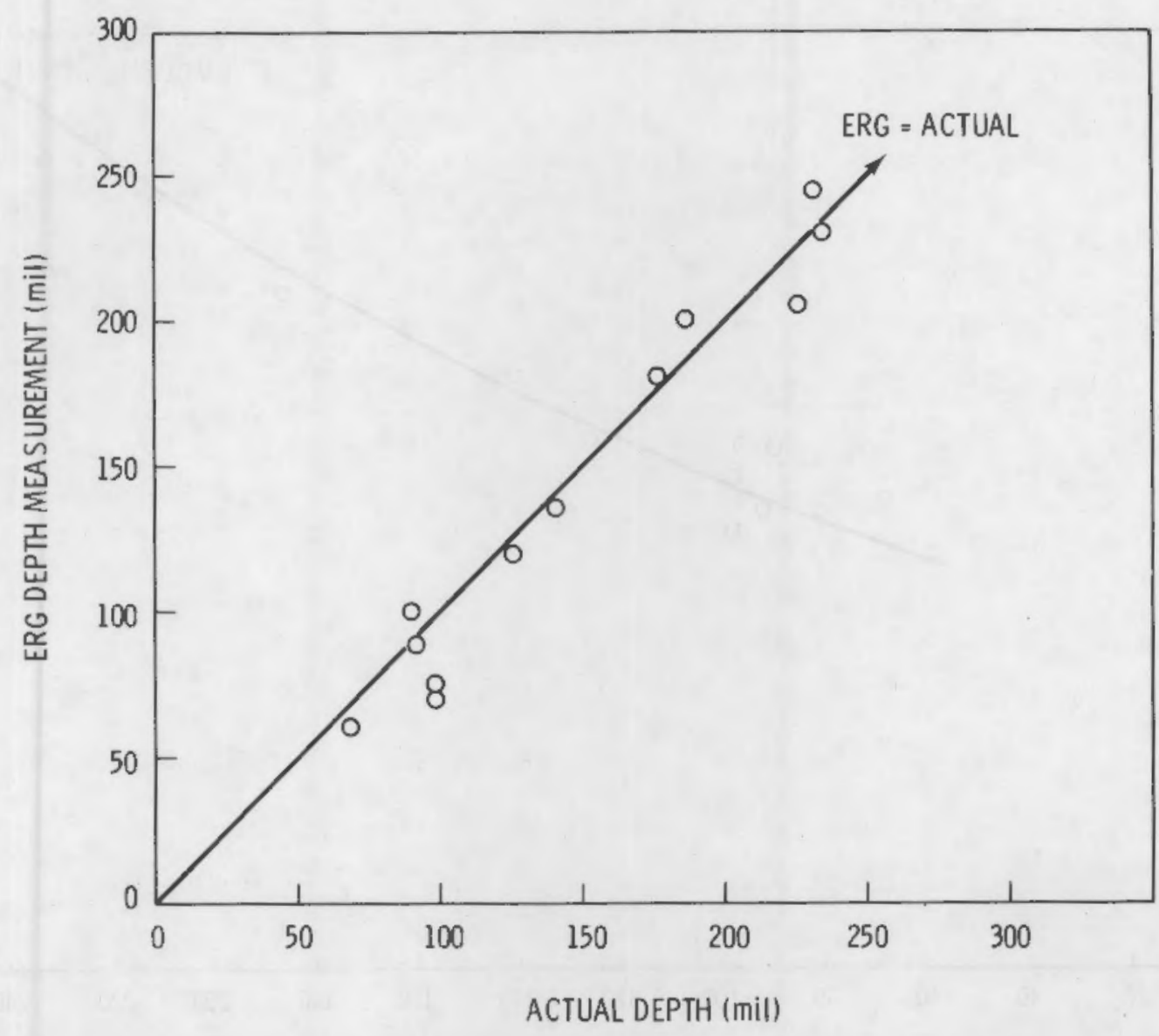

FIGURE 7.22. Electrical Resistance Gauge Performance in Measurement of Bending Fatigue Crack Depth

logarithmic, and power curves. Using the correlation coefficient as the criterion, the best correlations obtained were: 1) depth as a linear function of the logarithm of the $-6 \mathrm{~dB}$ probe motion in the shadow technique, resulting in a standard deviation of error in predicting depth of $17.6 \mathrm{mils}$, and 2) depth as an exponential function of the $-20 \mathrm{~dB}$ probe motion of the $1 / 2-\mathrm{in} ., 5-\mathrm{MHz}$ focused transducer, resulting in a standard deviation of error in predicting depth of 22.7 mils. See Figures 7.23 and 7.24 . The method most commonly used in the field, $-6 \mathrm{~dB}$ probe motion in pulse echo mode, produced very poor correlations.

Echo amplitude cannot be relied upon for crack sizing because it is too sensitive to crack tightness, roughness, orientation, and selection of transducer and inspection instrument. 


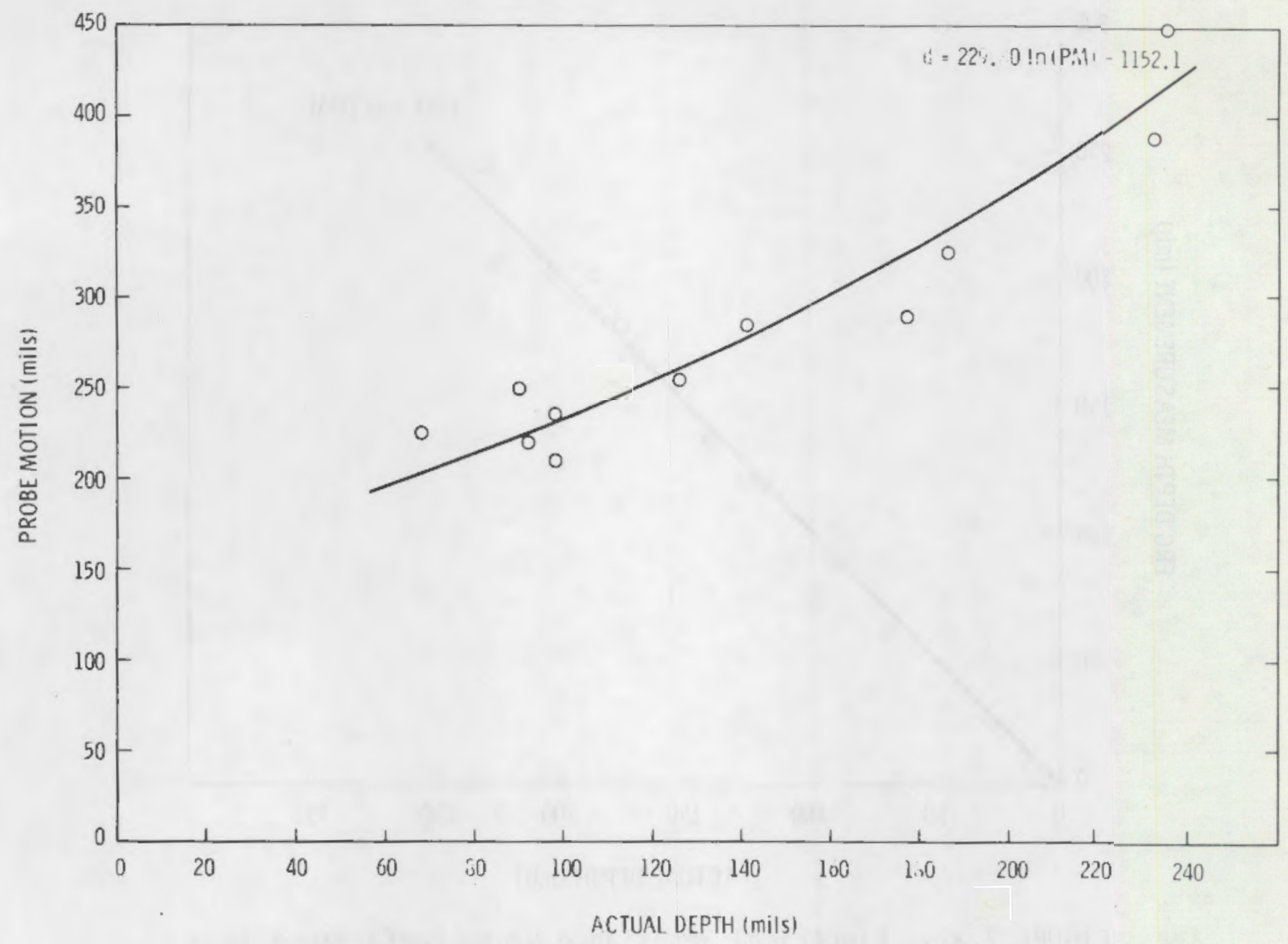

FIGURE 7.23. - $6 \mathrm{~dB}$ Probe Motion in Shadow Technique Inspection of Bending Fatigue Cracks

\section{Bending Fatigue Flaw Characteristics Effects on Detectability}

The analysis of uitrasonic inspection sensitivity to flaw orientation was performed using data obtained from EDM slits of known geometry. In order to predict the ultrasonic behavior of cracks in the same geometry, some direct comparison must be available between the responses of cracks and similar EDM slits. Such a comparison is available, for the bending fatigue cracks are closely modeled by the normal vertical EDM slits. They are similar in aspect ratio, range of depths, orientation and flatness of faces. Figure 7.25 shows ultrasonic reflection amplitude versus depth for both the EDM slits and the bending fatigue cracks. The actual depth is plotted for those cracks examined destructively; for the other cracks, depth was determined from the observed 


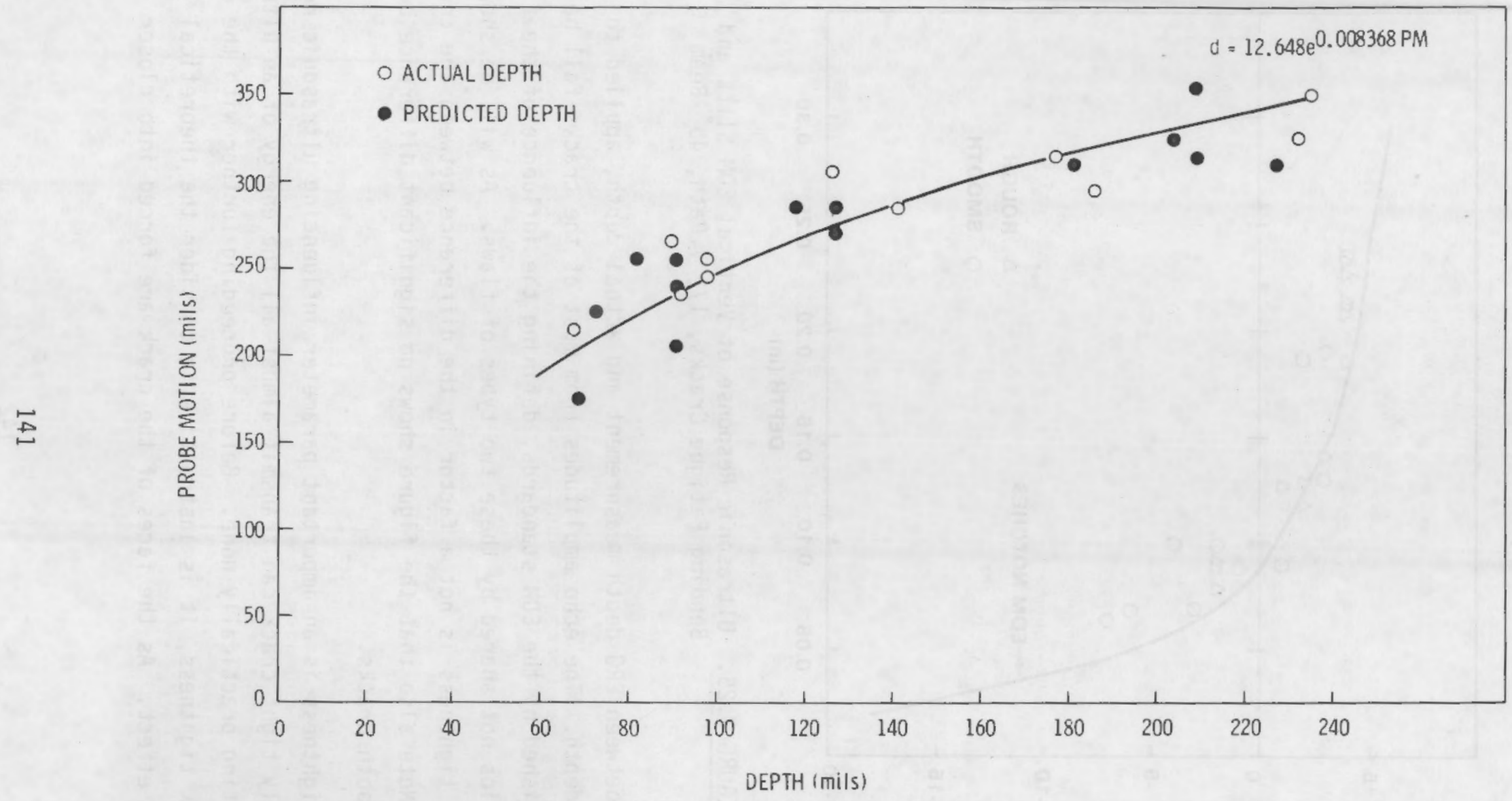

FIGURE 7.24. $-20 \mathrm{~dB}$ Probe Motion in Pulse-Echo Inspection of Bending Fatigue Cracks 


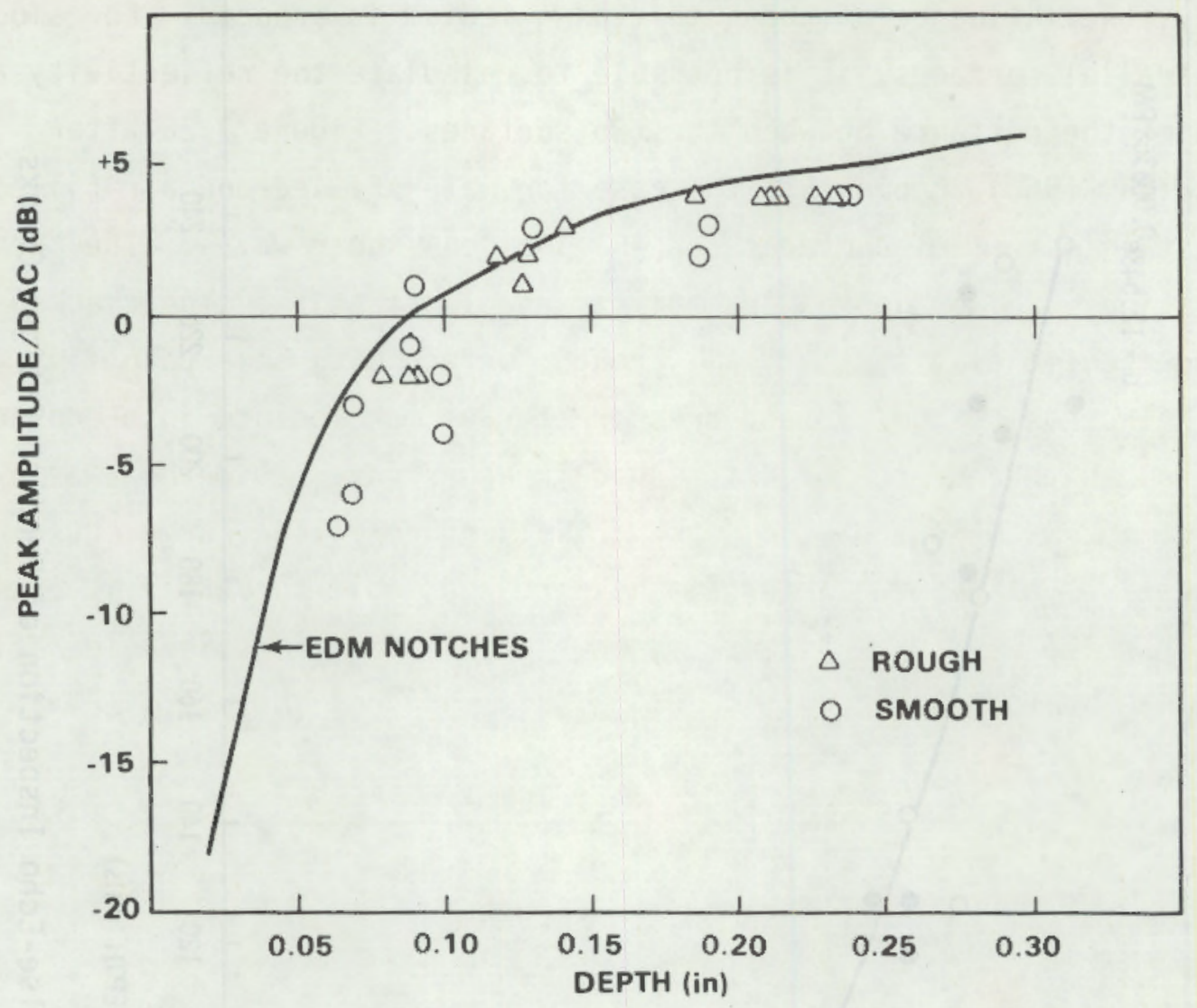

FIGURE 7.25. Ultrasonic Response of Vertical EDM Slits and Bending Fatigue Cracks, $1 / 2 \mathrm{~V}$ Path, $45^{\circ}$ Beam

correlation between ERG depth measurement and actual depth, applied to the cracks' ERG depth. The echo amplitudes from most of the cracks fall below the curve established by the EDM standards, defining the influence of the flaw characteristics not shared by these two types of flaws. As will be shown later, crack tightness is not a factor in the difference between the cracks and the slits. Note also that the figure shows no significant difference between rough and smooth cracks.

Crack tightness is an important parameter influencing ultrasonic response. A sufficiently tight crack can transmit almost all the energy of an ultrasonic wave, reflecting practically none. Before proceeding further with the discussion of crack tightness, it is instructive to consider the theoretical influence of this effect. As the faces of the crack are forced into closer 
proximity, the influence of the intervening medium is reduced. For smoothplane, parallel surfaces, it is possible to calculate the reflectivity as a function of the distance between the two surfaces. Figure 7.26 after Brekhovskikh (1960), represents the case for water-filled and air-filled gaps between stainless steel surfaces for $45^{\circ}$ incident shear waves. The theoretical considerations are mentioned here only as an illustration. The crack is more truly represented as a statistically rough surface with some points in intimate contact. Increasing the contact pressure forces more points into contact and results in a higher degree of ultrasonic transparency. No method was available to measure the tightness of the cracks, so the theoretical curve could not be verified quantitatively; however, means were available to verify it qualitatively. Particularly of interest was the lower limit of achievable reflection coefficient and the consequent impact on detection reliability.

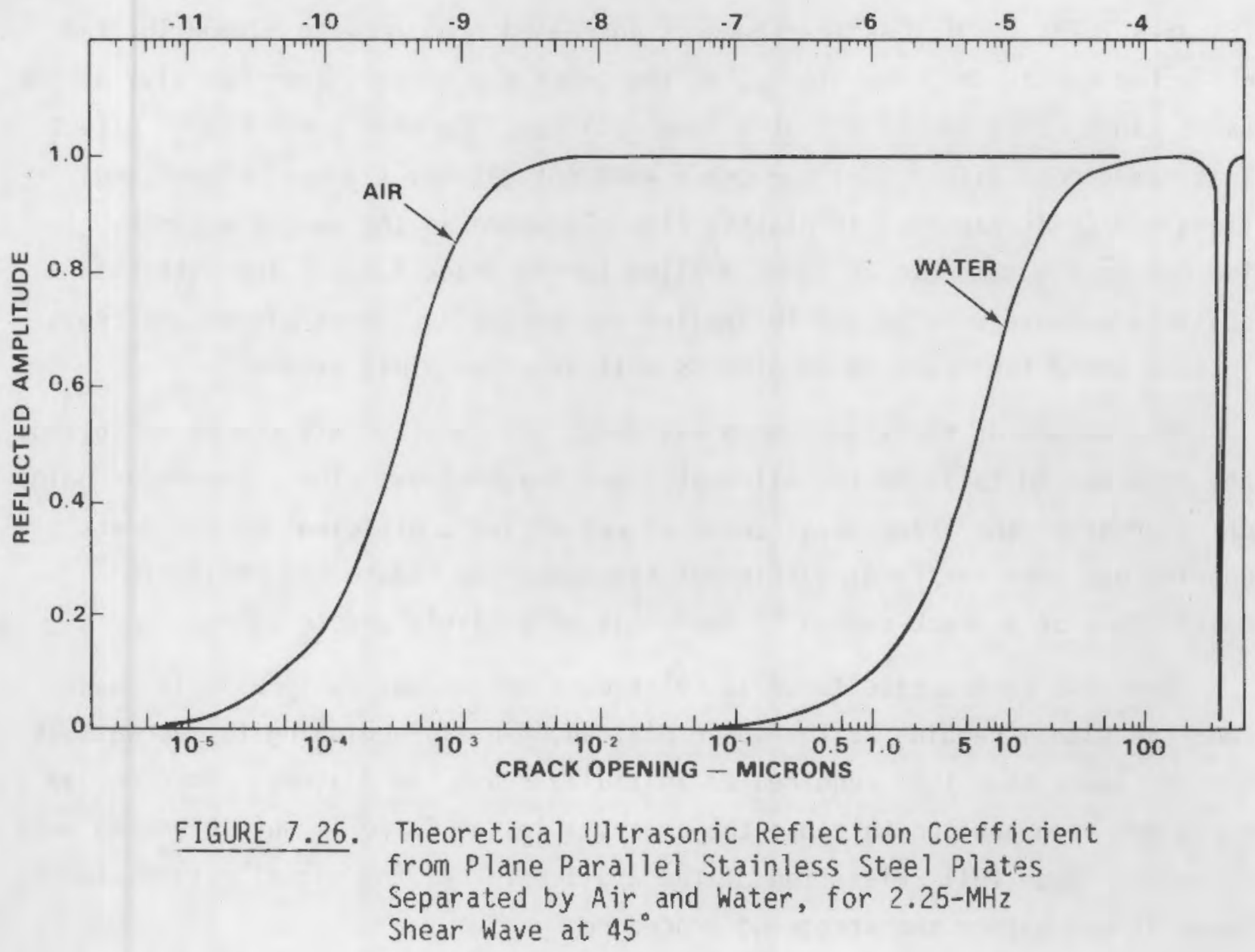


In order to vary the crack tightness, a four-point bending fixture was used (see Figure 7.27). This set-up allowed us to put the crack in tension or compression, and to position the transducer (1/2-in. dia, 2.25 -MHz contact unit) by hand for maximum response while under load. Ultrasonic response in $\mathrm{dB}$ relative to $D A C$ was measured as a function of force applied to the bending fixture. This force is not easily relatable to stress at the crack face, but is sufficient for the purpose of establishing the magnitude of the effect of tightness on ultrasonic response. The DAC was established by calibration on a milled $10 \%$ notch per ASME Section XI specifications.

Putting the cracks in tension, reducing the tightness, had no effect. This means that with no externally applied force the cracks were already fully open for the purpose of ultrasonic reflection.

When compressive force is applied, the reflection amplitude drops markedly (Figures $7.28,7.29$ ). As the force is increased from zero to $50,000 \mathrm{lb}$, the reflected amplitude drops slowly, as the crack tip closes, then rapidiy, as the faces close, then levels off at a lower plateau. Further force has no effect. This could mean either that the crack will not get any tighter or that additional force is expended in plastic flow elsewhere in the sample without increasing the compressive force applied to the crack faces. The latter is possible because with 50,000 ib applied the stress in the sample at the cross section where the crack is located is well over the yield stress.

The amount of the signal drop was about the same for all cracks so tested; the drop was 30 to $34 \mathrm{~dB}$ for all depths and roughnesses. Thus, the major point of interest of the theoretical curve of reflection coefficient versus crack opening has been verified; sufficient tightness can reduce the reflection coefficient of a crack enough to render it effectively undetectable.

When the compressive force is relaxed, a mechanical hysteres is is observed; the signal remains at the lower plateau, finally beginning to increase at a force lower than that required to initially reach the plateau. Once enough force has been applied to close the crack, a lesser force is sufficient to keep it there. Upon full relaxation of the applied force, the signal returns to the level it had before the stressing procedure. 


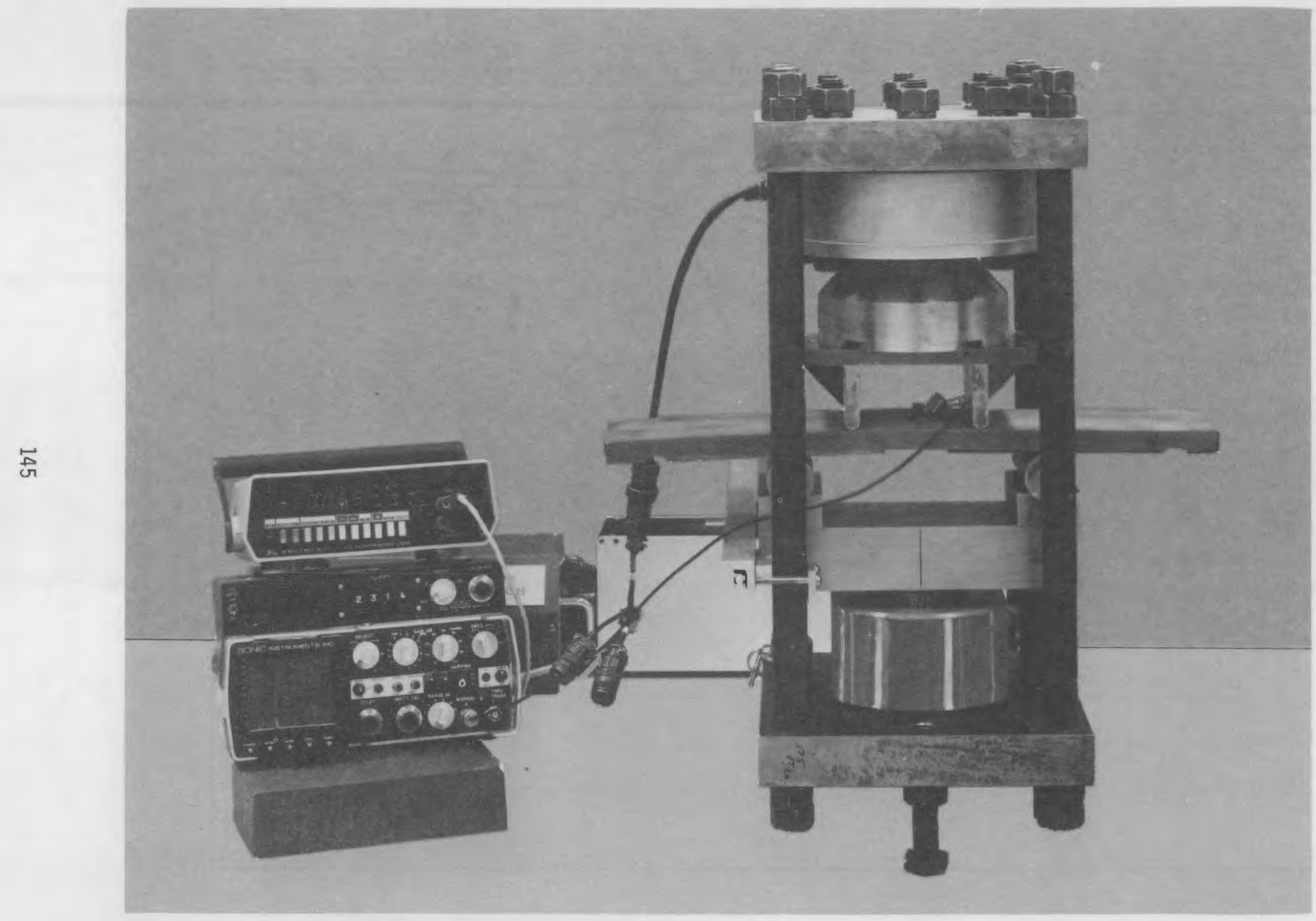

FIGURE 7.27. Four-Point Bending Fixture, UItrasonic Inspection Instrument, and Transducer 


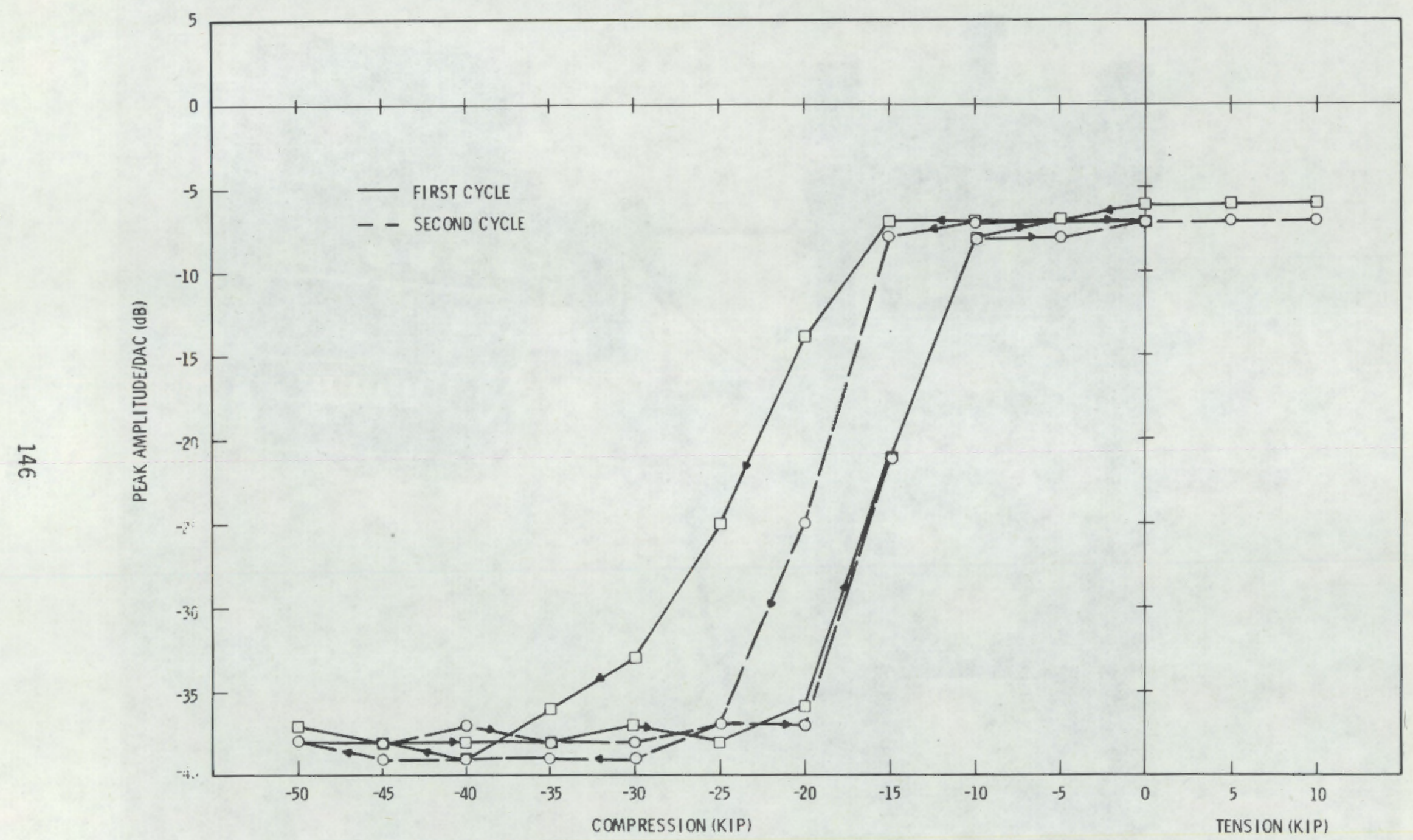

FIGURE 7.28. U1 trasonic Response of Bending Fatigue Crack A153 with Varying Force Applied to Four-Point Bending Fixture 


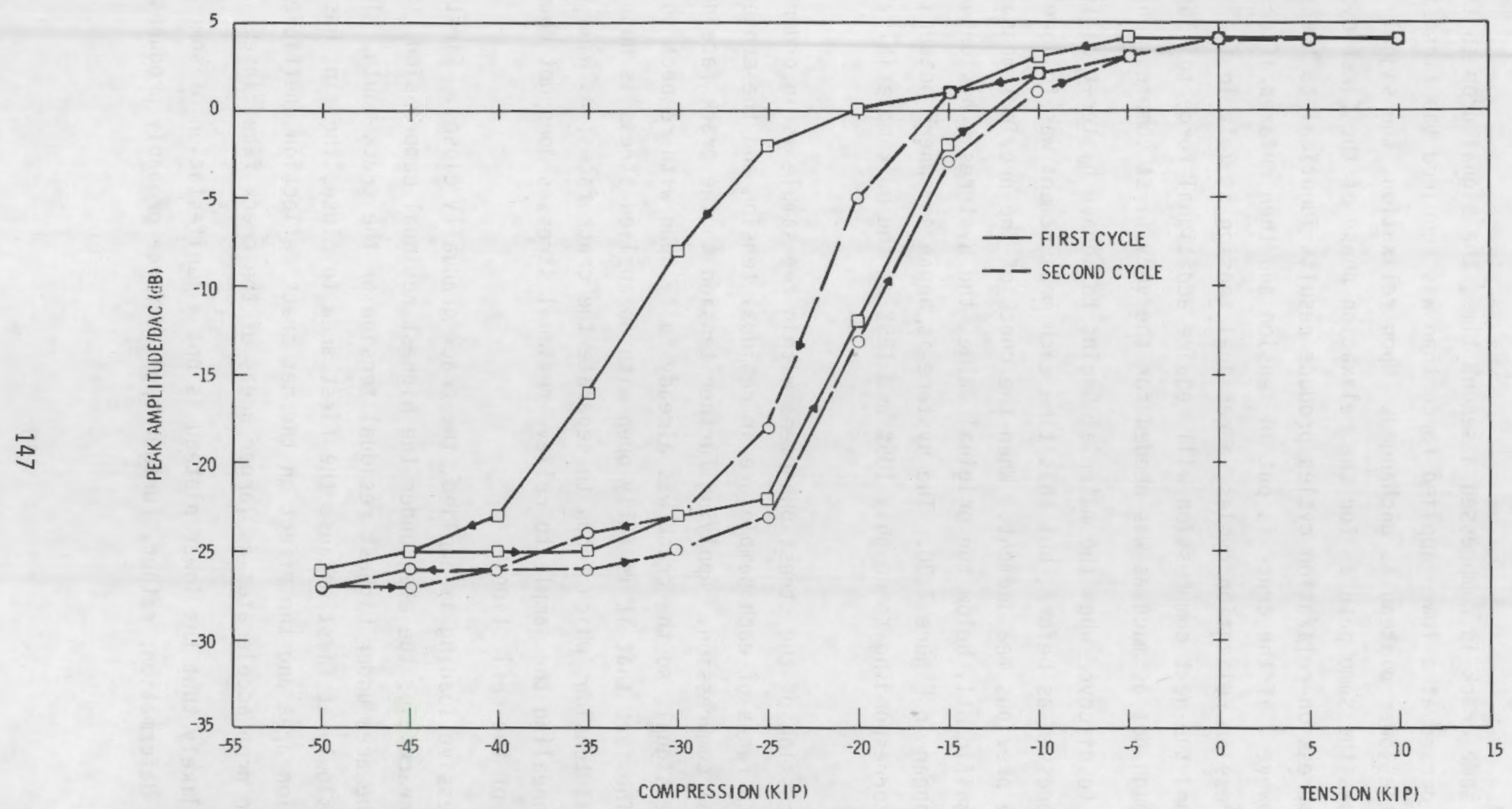

FIGURE 7.29. Ultrasonic Response of Bending Fatigue Crack Al61 with Varying Force Applied to Four-Point Bending Fixture 
When the same crack is compressed a second time, the signal drop to the lower plateau occurs at a lower applied force than was required the first time. The level of the lower plateau is unchanged. Upon relaxation, the signal increase follows the same path as for the relaxation phase of the first cycle. Subsequent compression-relaxation cycles produce results identical to the second cycle. However, if the crack is put in tension and then relaxed after one of these compression-relaxation cycles, a residual tension is left in the cracked face and the next compression will require additional force to close the crack (though not as much as was needed for the very first compression).

In order to discover when the material begins to behave hysteretically, a crack was compressed as before, but this time each measurement was followed by a check of the previous measurement. When the check of the previous measurement falls significantly below the original value, the hysteres is has begun. The results appear in Figure 7.30. The hysteresis began somewhere between the force levels corresponding to roughly $105 \%$ and $125 \%$ of the bulk material yield stress.

Some discussion of the stress phenomena within the sample is in order. Initially, both faces of each sample are in residual tension and the centers are in residual compression. Applying further tension to the crack face does not change the signal, so the crack was already fully open with respect to ultrasound. The fact that it is fully open with no applied stress is not due to the residual tension, which tends to separate the crack faces; as will be seen later, annealing the sample to relieve residual stresses does not change the amplitude of the reflection.

As compressive loading is applied, the crack gradually closes. First to close is the crack tip, the area under the highest residual compression. Last to close is the area under highest residual tension or the crack mouth. The signal drops slowly at first because the first area to close, the tip, has a small reflection area and the effect on the net crack reflection coefficient is small. The drop accelerates as larger areas of the crack face close.

It seem likely that the lower plateau is not a manifestation of some limit on crack face deformation; rather, further applied force probably produces 


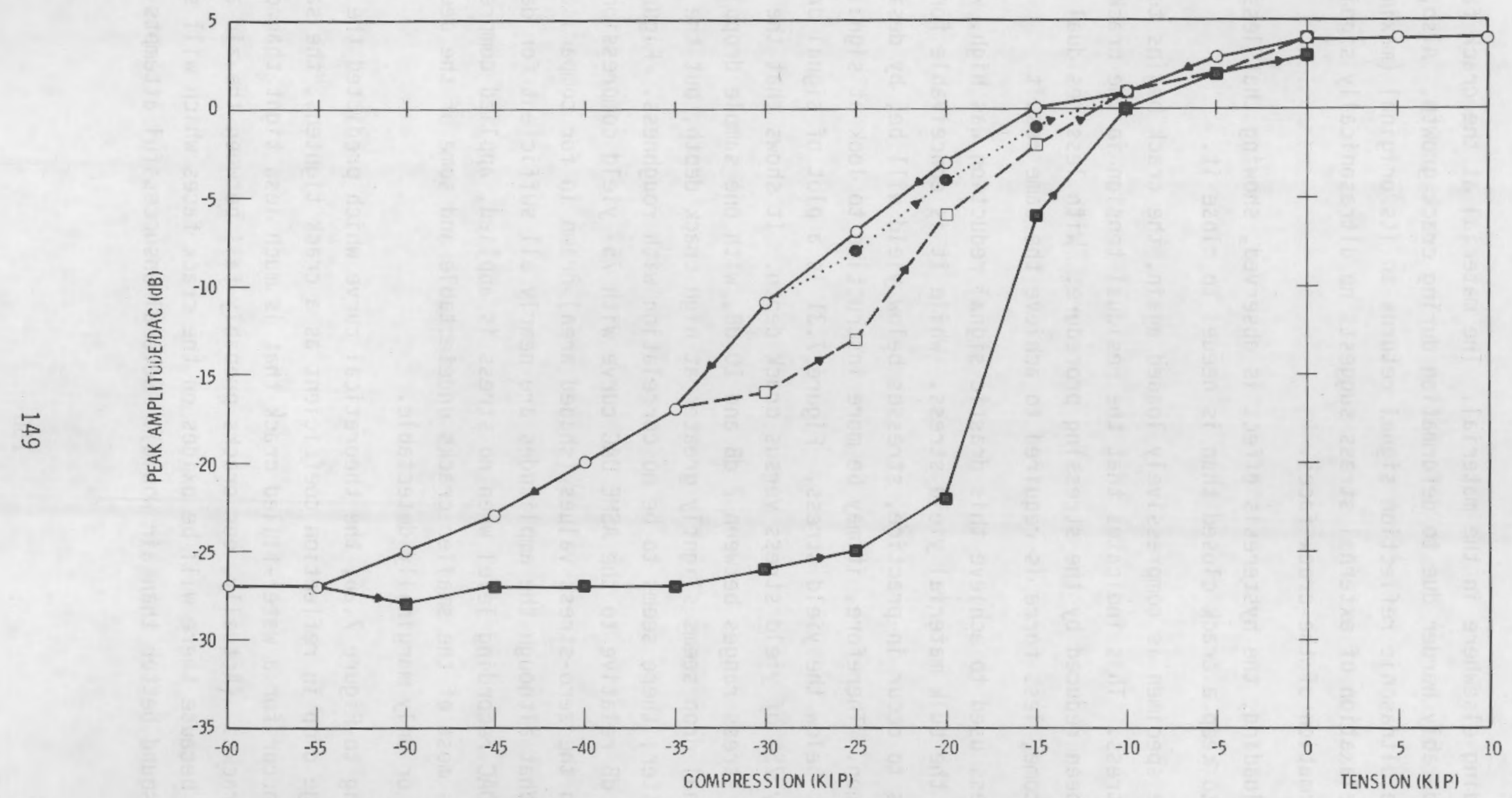
FIGURE 7.30. Ultrasonic Response of Bending Fatigue Crack A141 with Varying
Force Applied to Four-Point Bending Fixture 
plastic yielding elsewhere in the material. The material at the crack faces may be considerably harder due to deformation during crack growth. Also, the fact that the ultrasonic reflection signal returns to its original (maximum) value upon relaxation of external stress suggests no ultrasonically significant plastic deformation of the crack faces.

Upon unloading, the hysteresis effect is observed, showing that less force is required to keep a crack closed than is needed to close it.

When the specimen is compressively loaded again, the crack begins to close at a lower stress. This indicates that the residual tension in the cracked surface has been reduced by the stressing procedure. With less residual tension to overcome, less force is required to achieve the same result.

The stress used to achieve this drastic signal reduction was high, well in excess of the bulk material yield stress. While it is conceivable for such high stresses to occur in practice, stresses below yield will be, by design, far more common. Therefore, it may be more instructive to look at signal drop at some point below the yield stress. Figure 7.31 is a plot of signal drop at approximately $75 \%$ of yield stress versus crack depth. It shows that the signal drop at this stress ranges between $2 \mathrm{~dB}$ and $10 \mathrm{~dB}$, with one sample dropping 17 $\mathrm{dB}$. The signal drop seems slightly greater at high crack depth, but the data has much scatter; there seems to be no correlation with roughness. Figure 7.32 plots signal $d B$ relative to the ASME DAC curve with $75 \%$ yield compression applied, with the zero-stress values (shaded area) drawn in for comparison. It is clear that although the amplitudes are nearly all sufficient for detection at $50 \%$ DAC recording level when no stress is applied, applied compressive force renders most of the smaller cracks undetectable and some of the deep ones undetectable or only marginally detectable.

According to Figure 7.26 , the theoretical curve which predicted the observed very large drop in reflection coefficient as a crack tightens, the same effect will occur for a water-filled crack that is much less tight than our air-filled cracks. (Actually, our cracks probably fall between the air and water cases, because there will be oxides on the crack faces which will serve to transmit sound better than air would). Several unsuccessful attempts were 


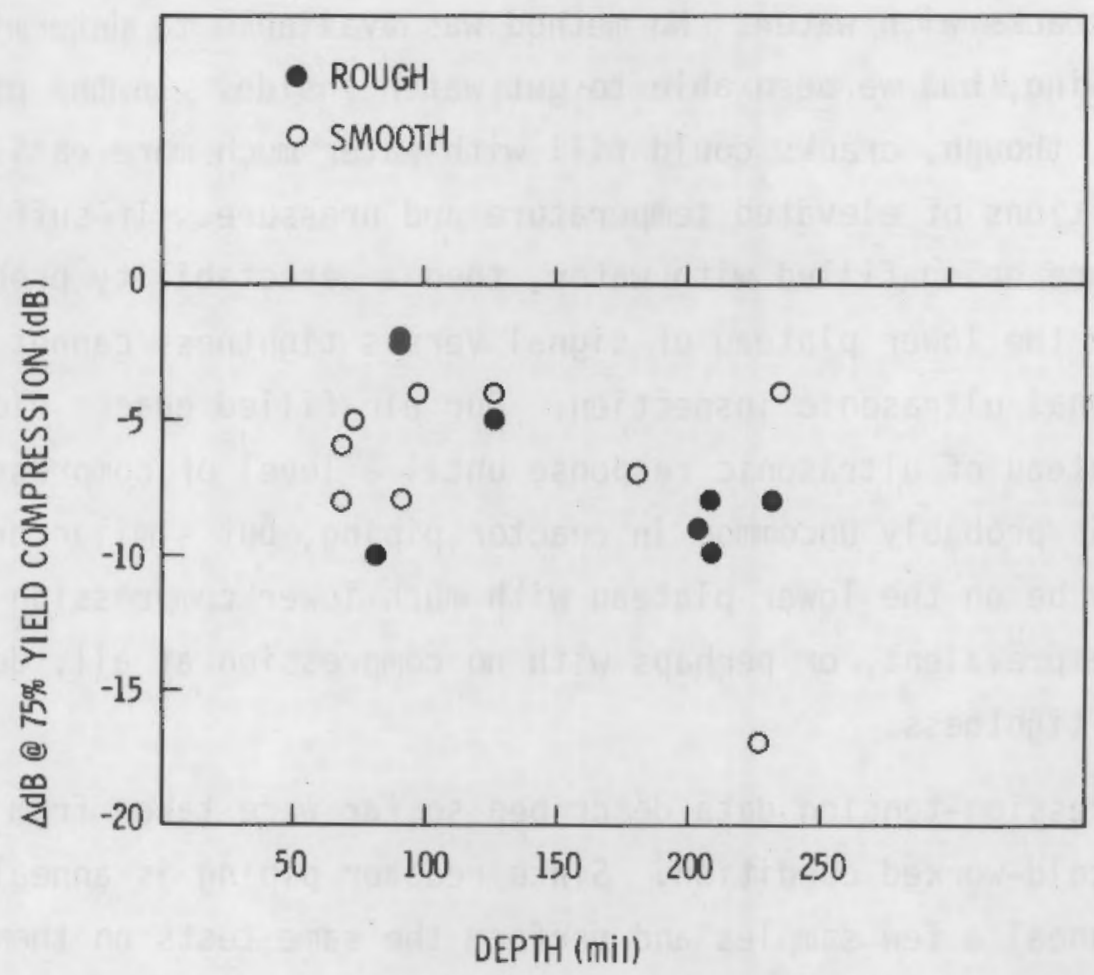

FIGURE 7.31. Change in U7trasonic Response of Bending Fatigue Cracks Upon Compression to $75 \%$ of Yield Stress

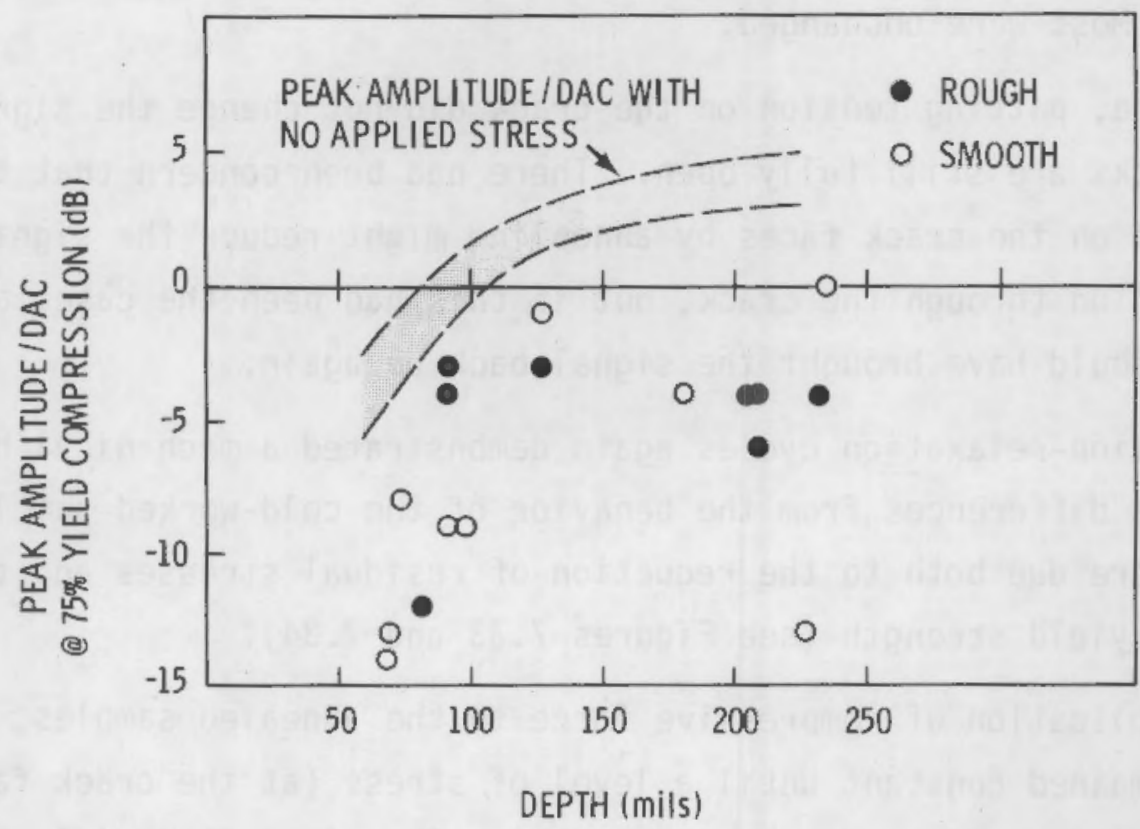

FIGURE 7.32. U1trasonic Response of Bending Fatigue Cracks Compressed to $75 \%$ of Yield Stress 
made to fill cracks with water. No method was available to determine the degree of filling, had we been able to get water inside. In the piping of a nuclear plant, though, cracks could fill with water much more easily at the existing conditions of elevated temperature and pressure. If sufficiently tight cracks are being filled with water, then a detectability problem exists, for a crack on the lower plateau of signal versus tightness cannot be detected by a conventional ultrasonic inspection. Our air-filled cracks did not reach this lower plateau of ultrasonic response until a level of compression was imposed that is probably uncommon in reactor piping, but similar cracks filled with water may be on the lower plateau with much lower compression forces, which are more prevalent, or perhaps with no compression at al1, depending on initial crack tightness.

The compression-tension data described so far were taken from samples still in the cold-worked condition. Since reactor piping is annealed, the next step was to anneal a few samples and perform the same tests on them, to see if the pronounced effect of crack tightness would still be evident.

Six samples were annealed. Annealing did not change the signal amplitude at zero applied stress significantly; the maximum change was a 2-dB drop for one sample. Most were unchanged.

As before, putting tension on the crack did not change the signal. The annealed cracks are still fully open. There had been concern that the oxide film produced on the crack faces by annealing might reduce the signal by allowing transmission through the crack, but if this had been the case, application of tension should have brought the signal back up again.

Compression-relaxation cycles again demonstrated a mechanical hysteresis, but there are differences from the behavior of the cold-worked samples. The differences are due both to the reduction of residual stresses and to the reduction of yield strength (see Figures 7.33 and 7.34 ).

Upon application of compressive force to the annealed samples, the signal amplitude remained constant until a level of stress (at the crack face) of about 75 of the yield strength was reached. Then it fell rapidly to a lower 


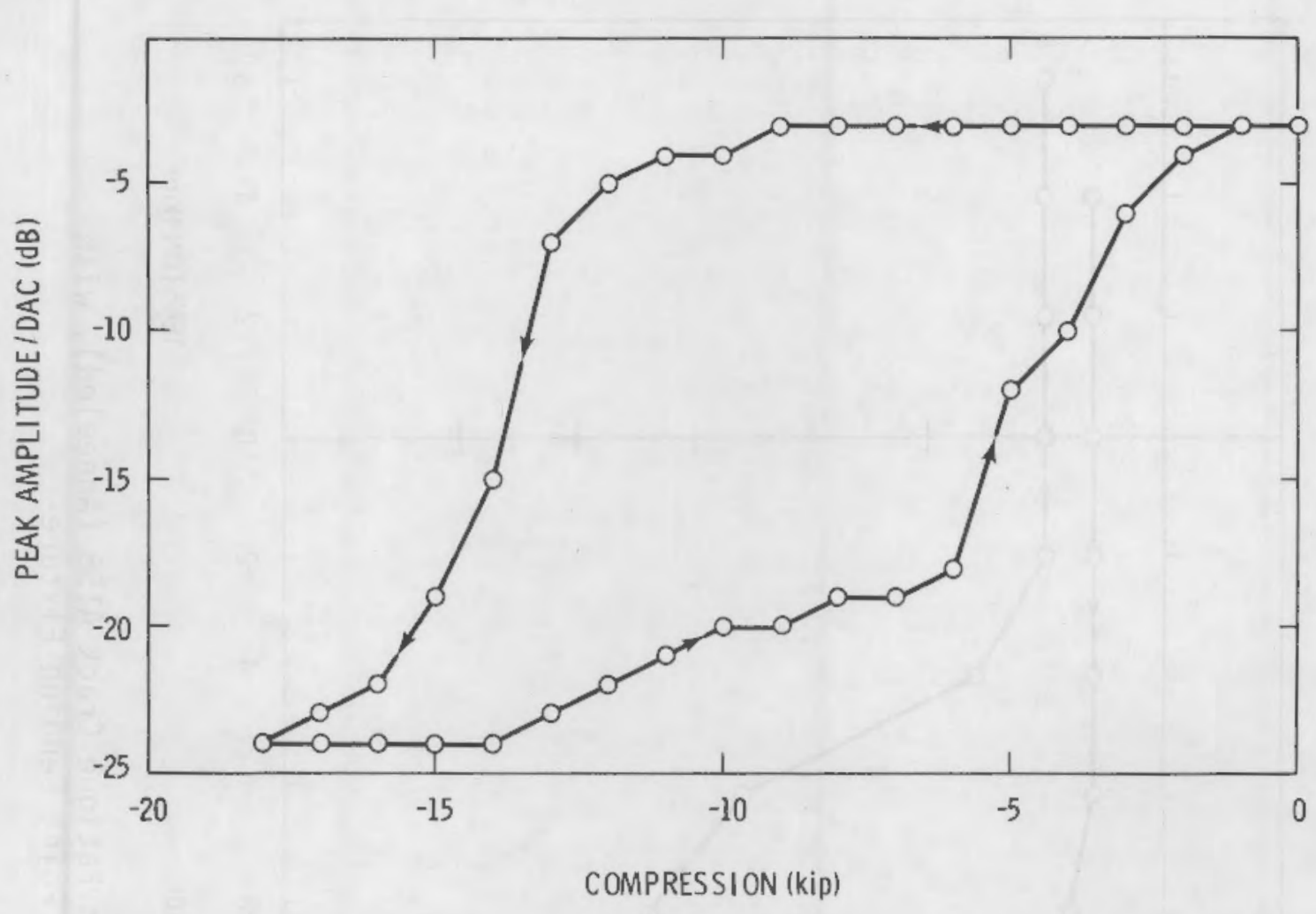

FIGURE 7.33. U1trasonic Response of Bending Fatigue Crack A155 (Annealed) with Varying Force Applied to Four Point Bending Fixture

plateau, which for all samples lay 21 to $23 \mathrm{~dB}$ beneath the zero-stress amplitude. As the applied force was relaxed, the signal remained at the lower plateau until, at a force lower than that necessary for crack closure, it increased slowly and then rapidiy to a value equal to or slightly less than the original. Application of tension at this point did not increase the signal further, regardless of whether it had fully returned to the original value.

The rapid signal drop observed when compression was applied occurred as the stress reached the neighborhood of the yield strength, for both the annealed and nonannealed samples. The reason that the annealed samples showed no gradual signal drop at lower stress is that, unlike the nonannealed samples, the crack tip was not under high residual compression. The drop was sudden because the crack closed all at once. 


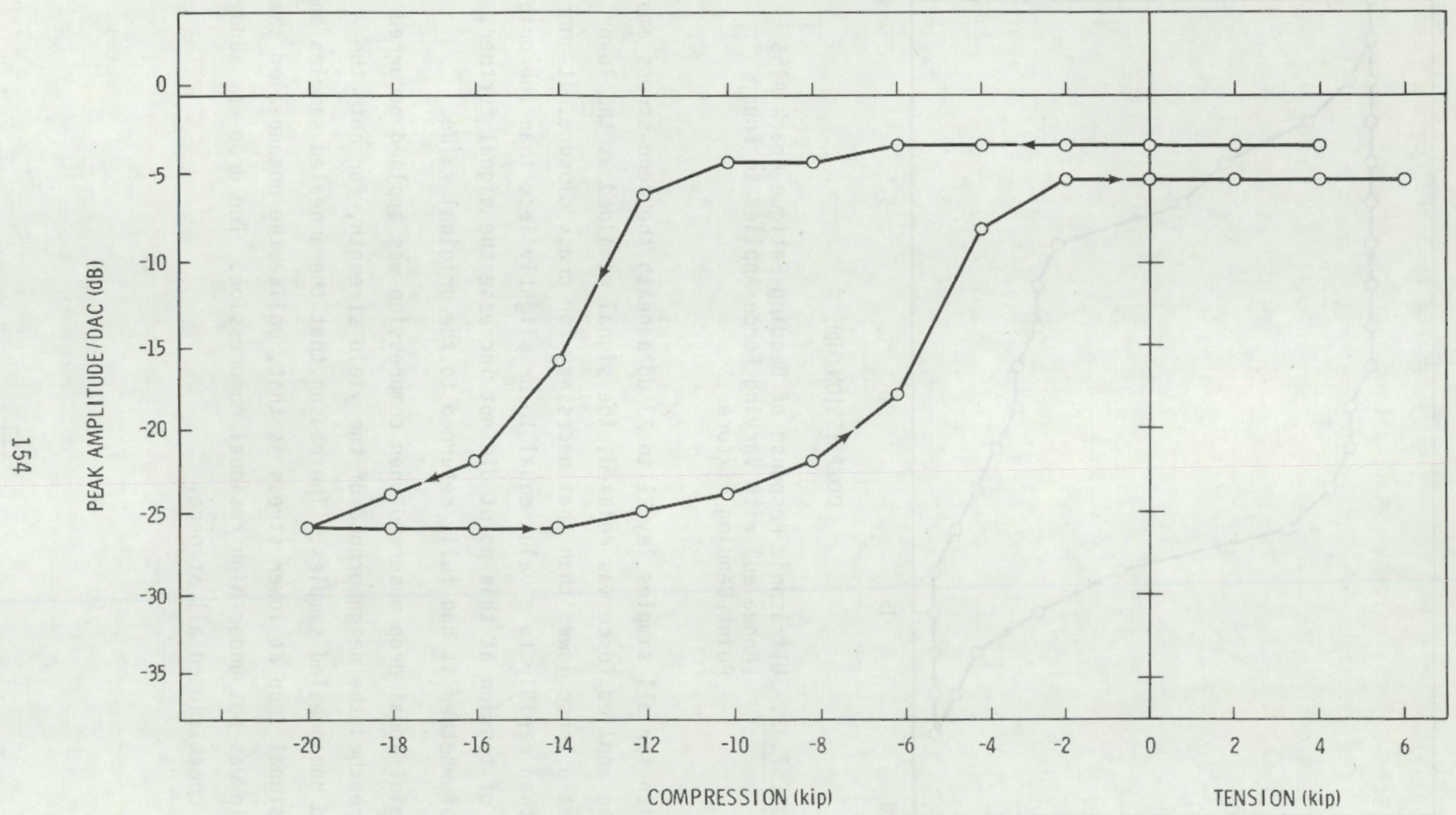

FIGURE 7.34. Ultrasonic Response of Bending Fatigue Crack A156 (Annealed), with Varying Force Applied to Four Point Bending Fixture 
The total signal drop was only $22 \mathrm{~dB}$, compared to $32 \mathrm{~dB}$ for the coldworked specimens. This indicates that, although crack closure occurred at the yield stress, the magnitude of signal decrease that is eventually achieved depends on the amount of force brought to bear on the crack faces. Much less force is available in the annealed samples, because the yield strength is only half that of the cold-worked samples.

The previous comments on the effect of water intrusion into the crack apply equally here. Although the signal drop occurred at a stress level that is probably unusual in the field, the drop might occur with little or no compression applied if the crack is filled with water. And though the drop was $10 \mathrm{~dB}$ smaller for annealed samples, it is still enough to reduce the reflection amplitude to the extent that an ultrasonic inspector would ignore it.

Flaw Measurement Results - Thermal Fatigue Samples

The thermal fatigue cracks are quite different from the bending fatigue flaws. Although the microscopic roughnesses (graininess of the crack surfaces) are about equal, the thermal fatigue cracks are mush rougher in the macroscopic sense; they ripple, twist, and change direction. One sample that was examined destructively had a crack that bent over so far that its deepest third was propagating parallel to the surface. These variable topographies make prediction and analysis of the cracks' ultrasonic behavior difficult but could also be beneficial in some circumstances. For example, a flat vertical flaw may be difficult to detect on some counterbores, but one of these thermal cracks in the same position could have areas with geometries favorable to detection.

\section{Crack Depth Sizing}

The same sizing methods used with the bending fatigue samples were used with the thermal fatigue samples. None of the four methods produced consistent accuracy.

The satellite pulse technique performed more poorly than it had for the bending fatigue cracks. No crack showed a recognizable satellite pulse, perhaps because the noise level was high. The high background noise is a result of the necessity for high gain in inspecting these flaws. 
The influences of tightness and off-normal geometry on ERG depth measurement would seem to be in opposite directions. Exteme tightness would allow current flow across the crack face and the increased current flow indicates a smaller crack. Off-normal geometry causes the current to take a circuitous route around the crack, causing a large deptn determination. Both effects are reflected in the data as shown in Table 7.3 .

\begin{tabular}{ccc} 
TABLE 7.3. & $\begin{array}{c}\text { ERG Depth Measurement Performance on } \\
\text { Thermal Fatigue Cracks }\end{array}$ \\
$\begin{array}{ccc}\text { Sample } \\
\text { No. }\end{array}$ & $\begin{array}{c}\text { Actual } \\
\text { Depth, mil }\end{array}$ & $\begin{array}{c}\text { ERG Depth } \\
\text { Measurement, mil }\end{array}$ \\
\cline { 1 - 2 } B101 & 250 & 135 \\
B103 & 193 & 200 \\
B104 & 158 & 400 \\
B114 & 147 & 120
\end{tabular}

The ERG measurement is low for two of four samples and about right for a third sample; the sample for which the ERG measurement is far too high is that sample whose crack propagation direction was farthest from vertical. These two competing effects, either one of which could dominate and cause significant error for a particular flaw, make the ERG depth measurement unreliable for these flaws.

Probe motion measurements are taken from a trace of echo amplitude versus transducer position. For EOM slits and bending fatigue cracks these curves are smooth and regular, but the traces obtained from thermal fatigue cracks are so irregular as to make measurements ambiguous or impossible. This is a result of variable off-normal crack geometry and (for through transmission) ultrasonic transmission through the crack.

Echo amplitude also fails; the cracks are so tight that their echo amplitudes (with no applied stress) fall an average of $18 \mathrm{~dB}$ below OAC. No correlation is observed between amplitude and destructively determined depth. Another influence disruptive to echo amplitude sizing is the nonplanar shapes the 
cracks assume; with so much variation in reflection geometry, echo amplitude will not be a simple function of through-wall penetration.

Thermal Fatigue Flaw Characteristics Effect on Detectability

The detectability of these flaws depends heavily on externally applied stress. The bending fatigue flaws had this dependence, but it became significant only with considerable applied compressive stress; the thermal fatigue cracks, though, are highly sensitive to applied stresses in the neighborhood of zero. At zero applied stress, all 12 cracks would be undetectable with $50 \%$ DAC recording criterion. The echo amplitudes ranged from $9 \mathrm{~dB}$ to $27 \mathrm{~dB}$ below $D A C$; mean and standard deviation are $17.9 \mathrm{~dB} \pm 5.7 \mathrm{~dB}$. The destructively measured cracks are all rejectable according to the criteria of ASME Boiler and Pressure Vessel Code, Section XI, Table IWB-3514-3; their depths are 147, 158,193 and $250 \mathrm{mils}$.

Through application of external stress, the thermal fatigue cracks also verify the theoretical behavior of reflection amplitude as a function of crack opening. A plateau of (comparatively) high amplitude reflection occurs with the crack in tension and a low-amplitude plateau is observed with the crack compressed. At rest (no externally applied stress), the cracks are in the lower part of the high-slope region between the plateaus and are, therefore, very sensitive to changes in crack face closure forces.

The difference between the tension and compression plateaus ranges between $11 \mathrm{~dB}$ and $35 \mathrm{~dB}$. Most $\mathrm{fall}$ in the $28 \mathrm{~dB}$ to $35 \mathrm{~dB}$ range; the others are $11 \mathrm{~dB}$, $14 \mathrm{~dB}$, and $20 \mathrm{~dB}$. The destructed samples included three with plateau separation of $11 \mathrm{~dB}, 14 \mathrm{~dB}$, and $28 \mathrm{~dB}$; the $11-\mathrm{dB}$ and $14-\mathrm{dB}$ cracks were much less planar than the 28-dB crack. This may mean that the nonplanar geometry of the cracks prevented their full closure, resulting in a high lower plateau. Both these nonplanar flaws were in nonannealed samples, as was the sample with 20-dB plateau separation (which was not examined destructively); this may indicate that the nonannealed samples are more likely to develop curving, nonplanar cracks.

Plots of signal amplitude versus censile/compressive applied force reveal little or no mechanica) hysteresis effect for the nonannealed samples (see 
Figures $7.35,7.36)$. This is because the hysteres is is a phenomenon of plastic strain and the plateaus are usually reached for the samples without exceeding the yield stress. Annealed samples behaved hysteretically, as shown in Figures 7.37 and 7.38. The upper reflectivity plateau was reached at approximately the yield stress, which for these annealed samples corresponds to $11 \mathrm{ksi}$ load. Upon relaxation of the tensile load, the signal began dropping just before full unloading and at zero load had dropped only a few dB. At this point, the signal was 10 to $20 \mathrm{~dB}$ higher than the initial signal. A somewhat higher (compressive) load was required to reach the lower plateau. Relaxation to zero load brought the signal back up into the range +3 to $-14 \mathrm{~dB}$ relative to the initial value.

Though these flaws are undetectable (50\% DAC criterion) with no externally applied load, applying tension equal to the yield stress makes most of them detectable. Clearly, if cracks of this tightness occur in reactor piping, their detection will strongly depend on the stress condition at the crack under cold shutdown conditions. If the cracks fill with water, they may be undetectable under any realistic stress condition. Also, if the stress values at a crack vary from one in-service inspection to the next, then meaningful interpretation of what is happening to the crack will be more difficult.

\subsection{INSTRUMENTATION}

Phase I measurements of the influence of search unit and instrument operating characteristics on inspection reliability have been limited to demonstrating that such effects are highly likely. The influence of search unit selection has been demonstrated in Section 7.1 of this report. A simple demonstration of the effects of instrument operating characteristics is described below.

In order to demonstrate that instrument characteristics could influence test results, a series of measurements were made using two different ultrasonic inspection instruments. Two portable instruments of different manufacture but of similar specification were selected. All other components of the system-cables, search unit (0.5 in. dia, 2.25 MHz), calibration standard, and couplant--were held constant. Measurements were made on the test blocks described 


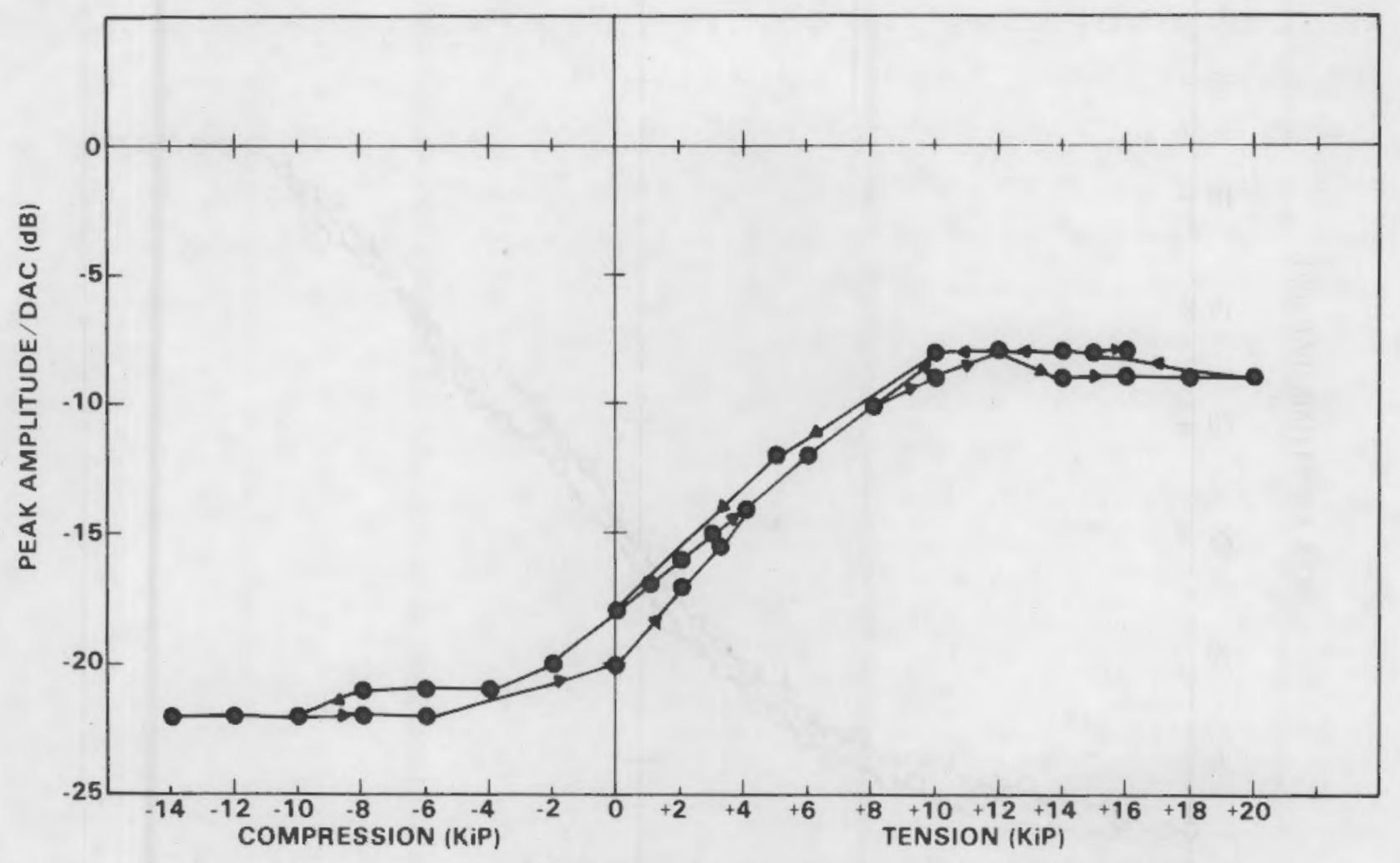

FIGURE 7.35. U1trasonic Response of Thermal Fatigue Crack B103 with Varying Force Applied to Four-Point Bending Fixture

in Section 7.1. The test response for the angled back surface notches is shown in Figure 7.39. Differences as large as $9 \mathrm{~dB}$ were measured. Response from instrument number 2 was consistantly higher by 2 to $4 \mathrm{~dB}$ for small cracks and off-angle notches, but approximately $1 \mathrm{~dB}$ lower for large vertical notches. The two instruments differ slightly in their pulse output and receiver bandwidth. When used with the same transducer, these conditions produce a center frequency difference of $0.3 \mathrm{MHz}(2.4$ to $2.1 \mathrm{MHz})$. The lower frequency of operation of instrument number 2 results in its lower sensitivity to flaw orientation conditions. Similar results could easily occur for different search units if their operating characteristics are not closely controlled. 


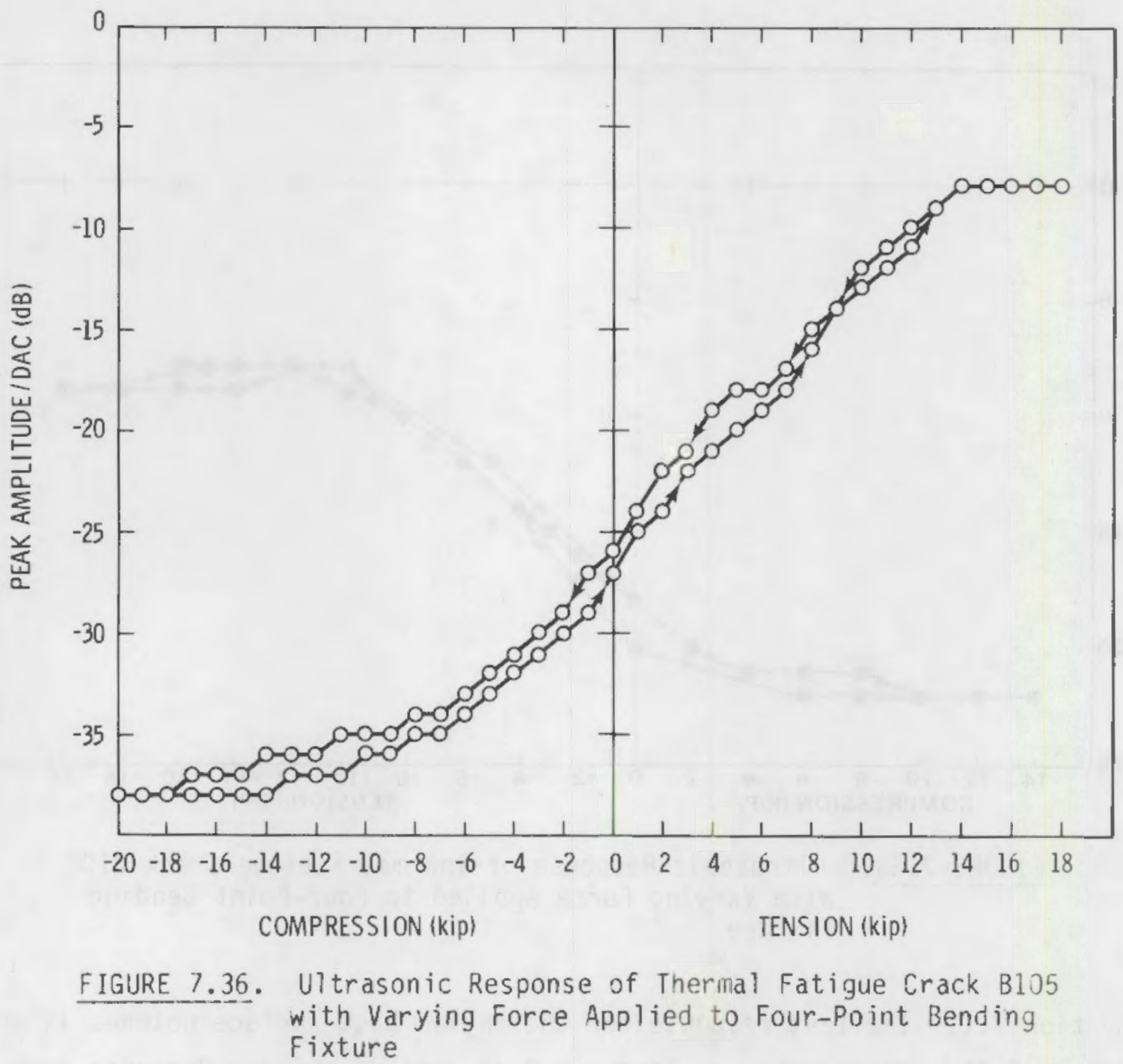

This demonstration indicates that inspection repeatability and reliability can be substantially influenced by the operating characteristics of the search unit and instrument. Investigations are continuing in this area to define the expected range of influence of instrumentation operating characteristics on inspection reliability. Recommendations for closer control of these characteristics will be developed.

\subsection{ESTIMATION OF FLAW DETECTION RELIABILITY}

A program (PISC 1979) is currently in progress under NRC sponsorship for the determination of failure probability of primary piping systems. These programs will use probabilistic fracture mechanics techniques for the failure 


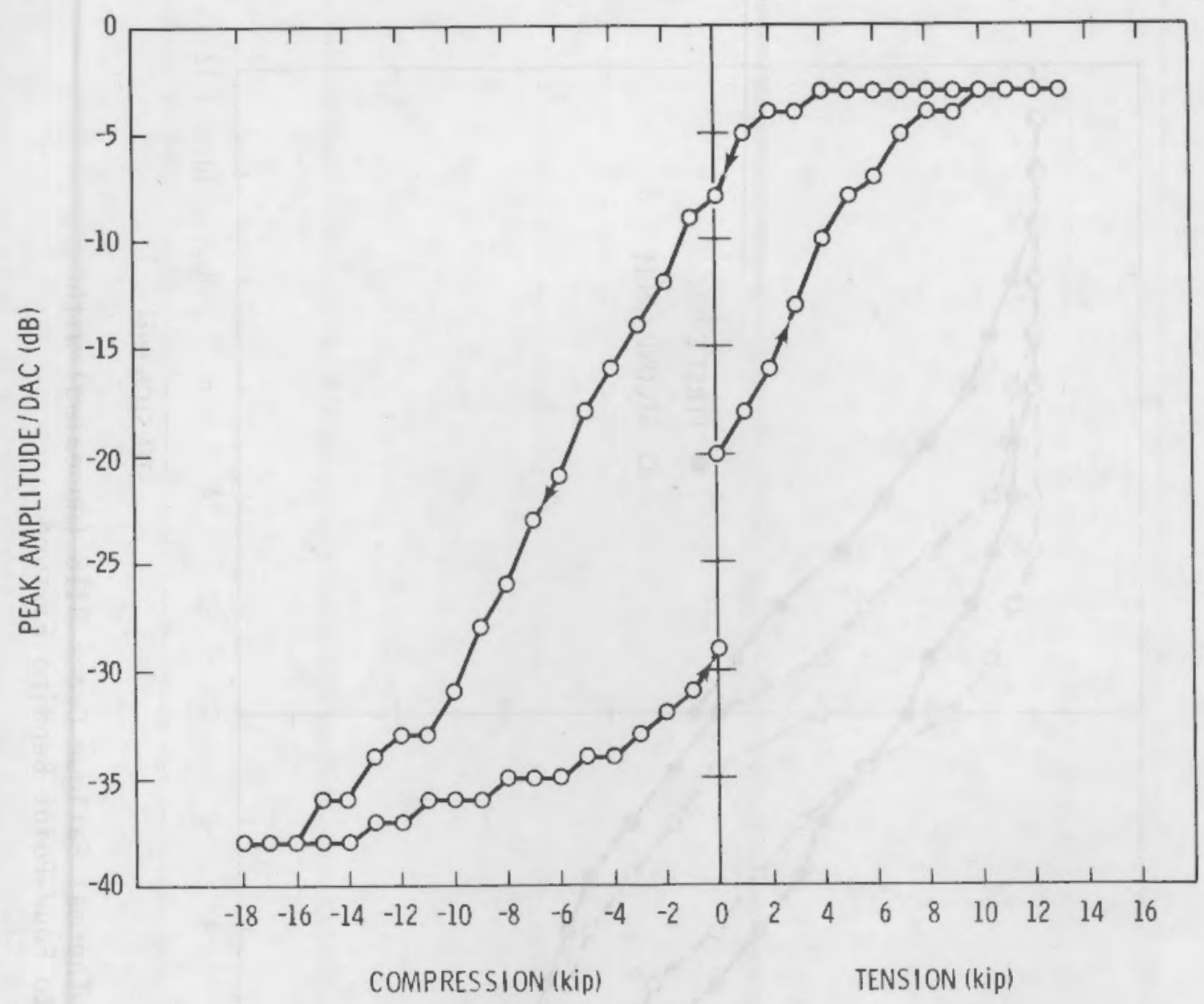

FIGURE 7.37. Ultrasonic Response of Thermal Fatigue Crack B113 (Annealed) with Varying Force Applied to FourPoint Bending Fixture

probability calculations. An important input parameter for these probabilistic techniques is the probability of correct rejection (POR) of a defect as a function of its through-wall depth. A round robin program for providing this POR data is in progress and is described in Section 5.2 of this report. In advance of the availability of this data in November 1982, it is necessary to provide realistic estimates of inspection reliability.

In the absence of measured POR data, we propose that a reasonable estimate of inspection reliability can be made based on an ultrasonic response model for predicting recording probability (RP), which is described below. The RP differs from POR in that two additional decisions must be made. These are: 


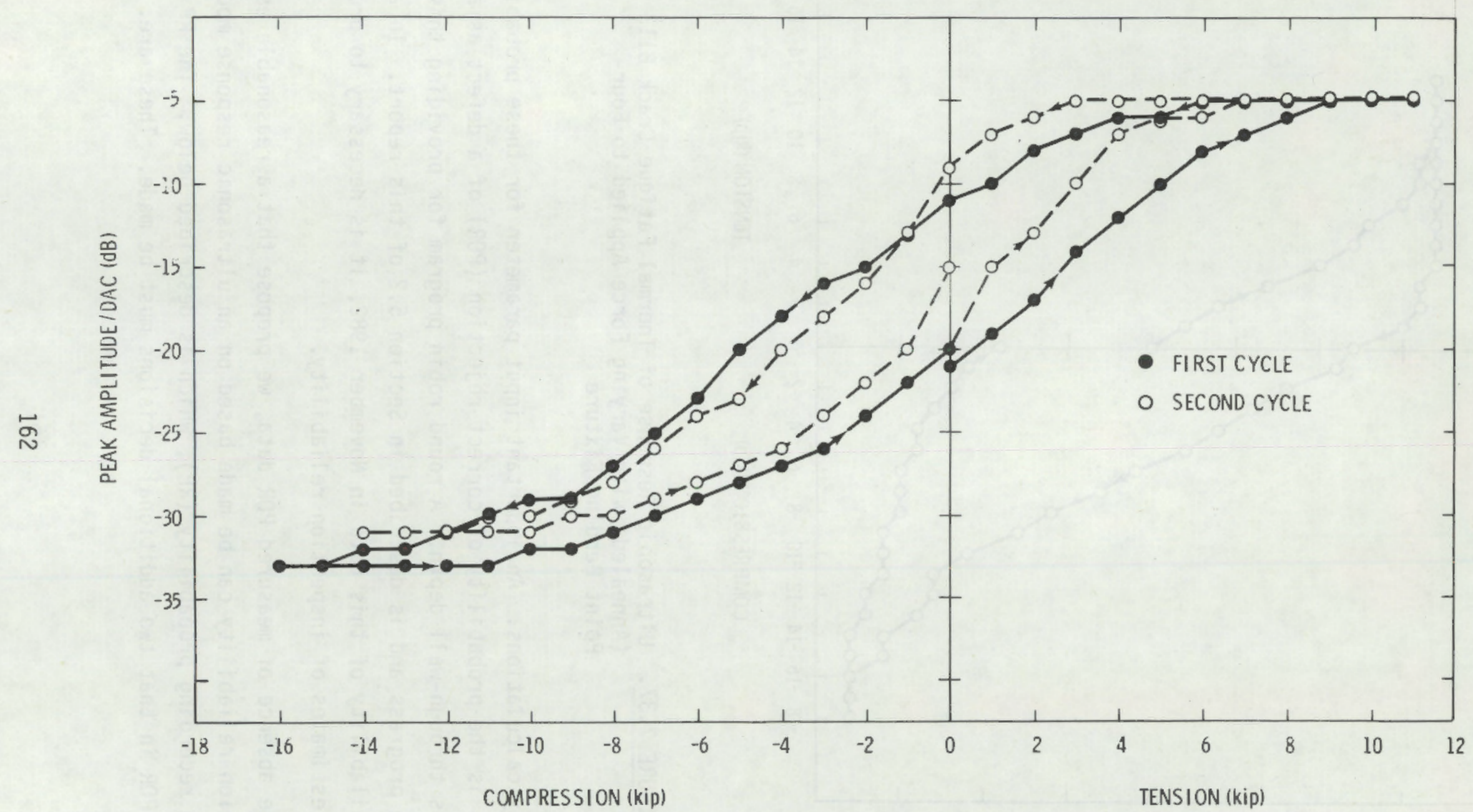

FIGURE 7.38. U1trasonic Response of Thermal Fatigue Crack B116 (Annealed) with Varying Force Applied to Four-Point Bending Fixture 


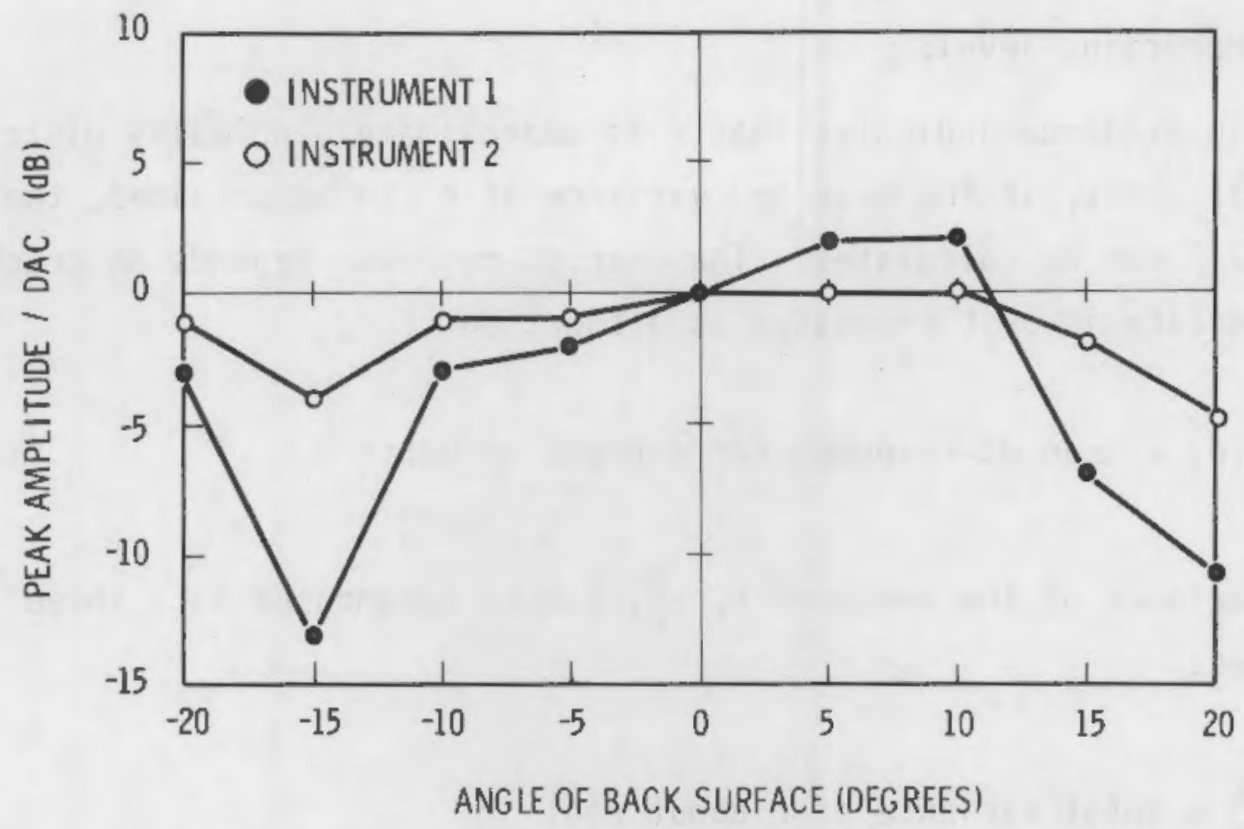

FIGURE 7.39. Response from 0.1 in. Deep Angled Back Surface Notches Using the Same Search Unit and Two Different but Similar UT Instruments

1) that the indication resulted from a crack and not one of the many geometrical discontinuities which are normally present, and 2) that it exceeds the allowable flaw size listed in Section XI of the ASME Code. Information regarding the efficiency of these last two decisions will only be available after completion of the round robin tests. In the mean time, the estimates given below should provide guidance for probabilistic fracture mechanics studies. Calculation of a Recording Probability Curve from dB-Response Information

The probability of receiving a recordable indication from a single crack of a particular size is related to the distribution of $d B$ response. Let $\operatorname{RP}(d)$ represent the recording probability of a crack of depth $d$ and let $r$ represent the maximum $d B$-response received from this crack. Since the Code procedure specifies that an indication only be recorded if it is above $50 \%$ DAC, the following relationship must exist between the two quantities:

$$
R P(d)=\operatorname{Pr}(r \geq C)
$$


where $\mathrm{C}=$ recording level.

Certain evidence indicates that $r$ is approximately normally distributed (PISC 1979). Thus, if the mean and variance of $r$ can be obtained, the probability in 7-2 can be calculated. The mean dB-response depends on crack size, so this quantity is best expressed as a function of $d$,

$$
\mu(d)=\text { mean } d 8 \text {-response for a crack of depth } d
$$

The variance of the response $r, \sigma_{r}^{2}$, can be decomposed into three subvariances:

$$
\begin{aligned}
& \sigma_{r}^{2}=\text { total variance of } r \text { about } \mu(d) \\
& \sigma_{r}^{2}=\sigma_{1}^{2}=\sigma_{2}^{2}=\sigma_{3}^{2} \\
& \sigma_{1}^{2}=\text { crack-to-crack variability } \\
& \sigma_{2}^{2}=\text { variability between inspection teams } \\
& \sigma_{3}^{2}=\text { variability within inspections }
\end{aligned}
$$

This decomposition makes sense if the observed $\mathrm{dB}$-response is generated by the following model:

$$
r=\mu(d)+e_{1}+e_{2}+e_{3}
$$

where $e_{1}$ is the crack error $\operatorname{Var}\left(e_{1}\right)=\sigma_{1}, e_{2}$ is the team error $\operatorname{Var}\left(e_{2}\right)=\sigma_{2}^{2}$ and $e_{3}$ is the inspection error $\operatorname{Var}\left(e_{3}\right)=\sigma_{3}^{2}$.

The crack error is the departure in $\mathrm{dB}$-response from the mean caused by differences in crack shape, orientation, tightness, etc. The team error is the departure from the mean caused by differences between inspection teams. The inspection error, $e_{3}$, is the departure caused by any changes in inspection 
technique that occur from inspection to inspection (scrubbing pattern, couplant, calibration changes, etc). The important aspect of the above model that allows $\sigma_{r}^{2}$ to be decomposed into three subvariances is the fact that the errors $e_{1}, e_{2}$, and $e_{3}$ are additive.

Once $\sigma_{r}^{2}$ and $\mu(d)$ are obtained, it is a simple matter to calculate RP(d). The formula is:

$$
R P(d)=\frac{1}{\sqrt{2}} \int_{-\infty}^{Z_{0}} e^{-1 / 2 r^{2}} d r
$$

where $z_{0}=\frac{\mu(d)-c}{\sigma_{r}^{2}}$

Our estimate of the mean response curve $\mu(d)$ is based on 24 bending fatigue crack samples (described in Section 6) and their measured response (described in Section 7.2) under compressive loading of $75 \%$ of yield stress relative to undeflected material. While the $75 \%$ compressive stress may appear to be very conservative, it should be noted that the data is for dry cracks. Cracks filled with liquid (the condition applicable for ISI of ID surfaceconnected cracks), IGSCC cracks, and tight thermal fatigue cracks may produce larger losses in signal amplitudes with no external stress applied. The experimental data is shown in Figure 7.40 , along with the response curve for ideal reflectors of aspect ratio (length/depth) 0.2 and the fitted curve. The mean of the measured flaws is $7 \mathrm{~dB}$ below the ideal response curve with a standard deviation of $4 \mathrm{~dB}$. The $2 \sigma$ error bars for this measurement are also shown in Figure 7.40 .

Estimates for the various sources of inspection error resulting from this study are listed in Table 7.4 along with the estimates made by Silk (1973) and Forli (1979). The standard deviation for the overall inspection process is surprisingly close for each of the estimates even though they are derived from substantially different sources. The data reported by silk resulted from 


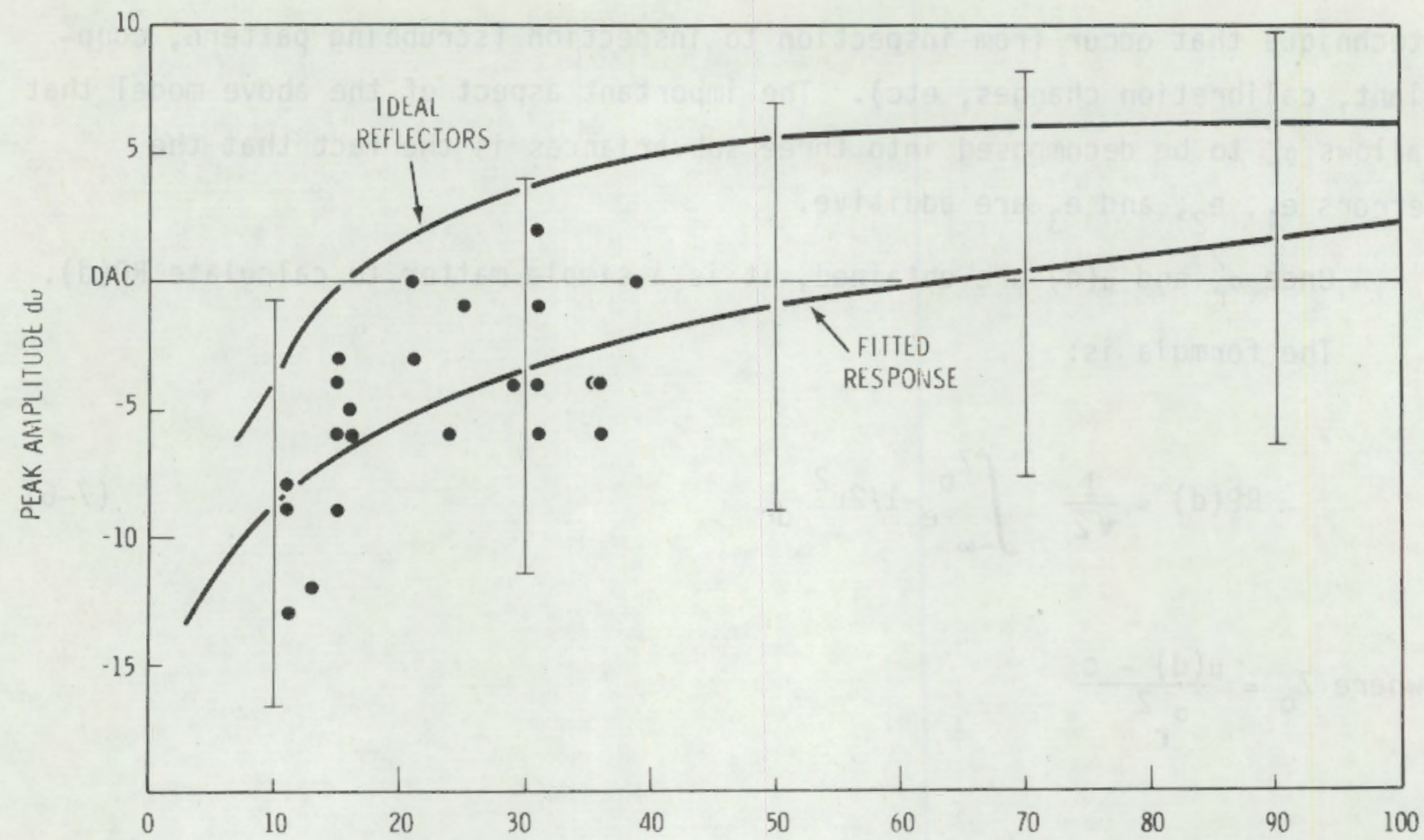

FLAW DEPTH PERCENI THROUGH WAI.I

FIGURE 7.40. Adjusted Response Curve for Fatigue Flaws Under Compression, Shown with 20 Error Bounds. The upper curve (ideal reflectors) is the response curve for notches of aspect ratio (depth/length) $0 . ?$

a compilation of approximately 16 different experiments on fatigue cracks in aluminum and steel. The data by For $1 i$ is the result of a round robin test on $70 \mathrm{~m}$ of mild steel butt welds of thickness 10 to $26 \mathrm{~mm}$.

The study reported by For $1 \mathrm{i}$ employed 12 inspection teams and $60 \mathrm{cracks}$, which were predominantly fabrication flaws reflecting lack of root penetration and lack of fusion.

In discussing the individual contributions to the total inspection error it should be noted that the three sources represent substantially different data sets and conditions. The within-inspection error is probably the most well documented of all the variables. Our data is based on approximately 500 
TABLE 7.4. Estimate of Standard Deviation of Inspection Variables

$\sigma_{3}$ within inspection

o2 between inspection teams coupling

$\sigma_{1}$ crack-to-crack total

Defect orientation

Defect roughness

Tr ansparency

$\sigma_{r}$ TOTAL INSPECTION
This Study

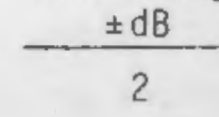

2

$-$

5.4

3.6

1.4

3.7

6.1
Silk (1978)

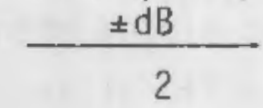

$-$

2

6.1

3.5

3.0

4.0

6.7
3.3

Forli (1979) $\pm \mathrm{dB}$

4.3

2.7
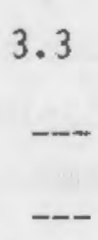

6.1

measurements under laboratory conditions, while Forli's 586 measurements represent field and laboratory conditions. Forli's data were collected at 10, 20 and $50 \%$ DAC, which may account for the higher within-inspection variability. Our estimate of $2 \mathrm{~dB}$, for between-inspector $\sigma$, is an engineering judgment based on the differences expected due to search unit and instrument selection; i.e., it is expected that $95 \%$ of the data will fall within an 8-dB band. Silk does not estimate between-inspector errors; however, the variable is accounted for in the total estimate. Silk also provides an estimate of $\underline{+2} \mathrm{~dB}$ due to coupling errors which are not separately evaluated by the other two studies.

The total error due to the flaw character is made up of the contributions due to defect orientation, roughness, and transparency. Only the total flaw contribution, $3.3 \mathrm{~dB}$, is available from the data of For li. Since the Forli data is based on fabrication flaws, the major contributors are most likely or ientation and roughness, with transparency having little influence. The estimates from our results are slightly less conservative than those presented by Silk, particularly in regards to flaw roughness effects. This difference exists partly because a portion of Silk's data was measured at $5 \mathrm{MHz}$ instead of the $2.25 \mathrm{MHz}$ used in our study. Increased frequency increases the scattering effect due to roughness. 
Before calculating the recording probability, it is necessary to define the linits of applicability for the estimates. The estimates are equally applicable to service-induced fatigue flaws in similar metal welds of wrought austenitic and ferritic piping where both sides of the weld are accessible. This assumes that the calibration procedure properly accounts for base metal attenuation. Due to the high attenuation and velocity anisotropy of austenitic and dissimilar metal welds, single-side access conditions using current inspection techniques will yield highly degraded results compared to two-side access. The applicable pipe wall thickness range is 0.5 to $2.5 \mathrm{in.}$, assuming that the most appropriate search unit is selected. This thickness range was verified by measurements on ideal reflectors over the required thickness range.

These calculations also assume at least a two-to-one signal-to-noise ratio. This condition is not satisfied by centrifugally cast stainless steel used in many of the Westinghouse reactors. The only estimate we can make in this case is an engineering judgment based on our experience with this material. If an optimized inspection procedure is used by an operator with substantial experience on centrifugally cast stainiess, we estimate that cracks up to $25 \%$ through-wall would have less than a $20 \%$ probability of detection, up to $50 \%$ through-wall less than $30 \%$ probability of detection and in no case greater than $50 \%$ probability.

The curves in Figure 7.41 represent our best estimate of recording probability (under the conditions listed above) for 20,50, and 100\% DAC recording thresholds for a total inspection standard deviation of $\pm 6.1 \mathrm{~dB}$. Current ASME Section XI requirements call for recording at or above 50\% DAC and reporting at or above $100 \%$ DAC.

Factors not considered in the estimate but which may effect their accuracy include: psychological factors, errors in identification, length of the defect, and interaction of variables. The length of the defect may be important as a long defect will present more opportunities for detection. This factor may result in our estimates being overly conservative for large flaws. The other factors will be investigated in the round robin and other Phase II efforts. 


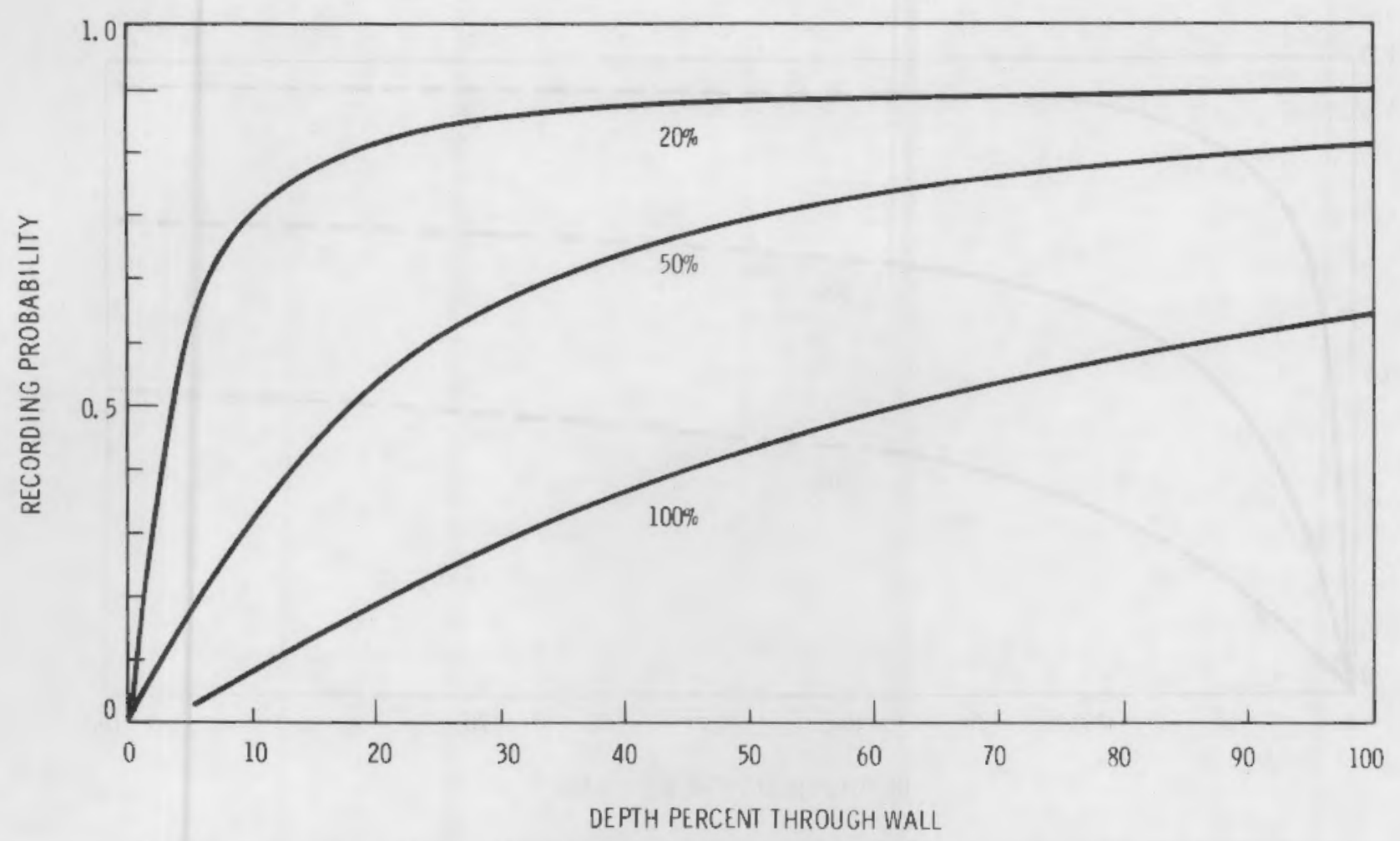

FIGURE 7.41. Estimated Recording Probability (i.e., the probability that the ultrasonic response will exceed a threshold value) Versus Flaw Depth for 100,50, and 20\% DAC Recording or Reporting Thresholds

The data by Forli represent controlled tests using 12 inspectors under field and laboratory conditions. The flaws considered were fabrication flaws such as lack of fusion and lack of root penetration. The tests were performed on weld joints from 10 to $26 \mathrm{~mm}$ in thickness with the results reported as a function of flaw depth rather than percent of thickness. If it is assumed that the thickness was $26 \mathrm{~mm}$ for all flaws (least conservative estimate) the curves of Figure 7.42 can be derived from Forli's data. If we had assumed $10 \mathrm{~mm}$ thickness, the data up to $36 \%$ through-wa!l thickness would represent $100 \%$ through-wall depth.

In comparing our estimates (Figure 7.41 ) with the data of Figure 7.42 we see that they are in substantial agreement. From this we can conclude that our estimates are not overly conservative. 


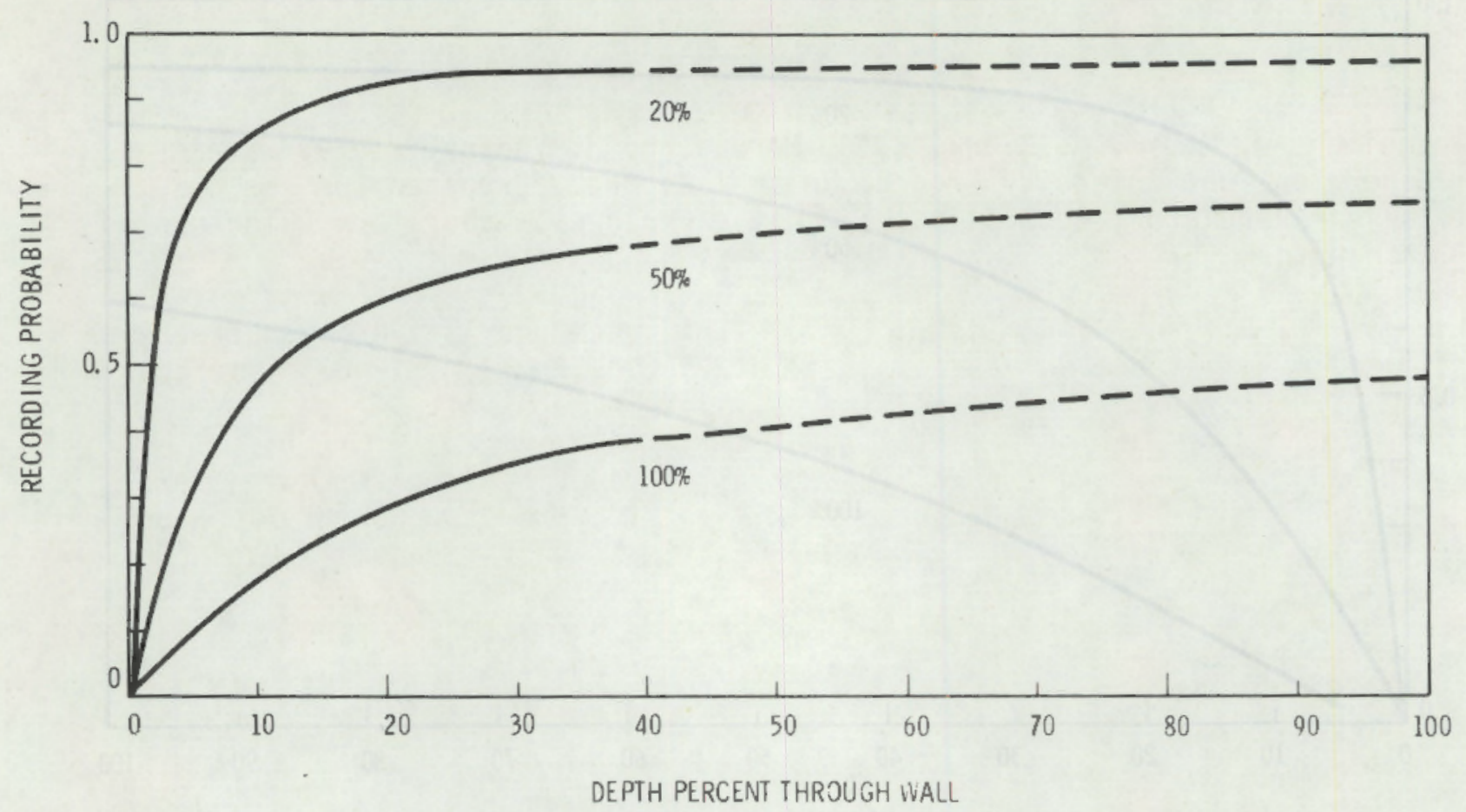

FIGURE 7.42. Measured Recording Probability Versus Depth for 100, 50, and 20\% DAC Thresholds Reported by Forli (1979).

Based on the predictions of Figure 7.41 and 7.42 it is apparent that the $100 \%$ DAC reporting level specified in Appendix 3 of ASME XI is not adequate to provide assurance of the weld integrity. 


\section{REFERENCES}

Alzheimer, J. M., R. A. Clark, C. J. Morris and M. Vagins. 1979. Steam Generator Tube Integrity Program Phase I Report. Prepared for the U.S. Nuclear Regulatory Comission by Pacific Northwest Laboratory, Richland, Washington.

Andrews, W. R., and C. F. Shih. 1979. "Thickness and Side-Groove Effects on $\mathrm{J}-$ and $\delta$-Resistance Curves for Steel at $93^{\circ} \mathrm{C} . "$ In ASTM STP-668, American Society for Testing and Materials, pp. 426-450.

ASME. 1977. ASME Boiler and Pressure Vessel Code, Section XI, Rules for Inservice Inspection of Nuclear Power Plant Components. Juty 1977, with addenda through winter of 1978. American Society of Mechanical Engineers.

ASNT. 1977. "Round Rob in Report," ASNT Sonics Committee Meeting Agenda Item 3. ASNT Spring Conference, March 1977.

Bamford, W. H. 1979. "Application of Corrosion Fatigue Crack Growth Data to Integrity Analyses of Nuclear Reactor Vessels." 79-PVP-116, paper presented at the ASME Pressure Vessels and Piping conference, June 25-29, 1979, San Francisco.

Bamford, W. H., and A. J. Bush. 1979. "Fracture Behavior of Stainless Steel." In ASTM STP-668, American Society for Testing and Materials, pp. 553-580.

Birks, A. S., and W. E. Lawrie. 1978. "Search Unit Specification for Improved Repeatability of Ultrasonic Examinations." In Proceedings of an International Conference on Nondestructive Evaluation on the Nuclear Industry.

Bishop, I. M., S. E. Feinberg and P. W. Holland. 1975. Discrete Multivariate Analysis. MIT Press, Massachusetts.

Brekhovskikh, L. M. 1960. Waves in Layered Media. Academic Press, New York, NY.

Buchanan, R. A. 1975. "Analys is of the Nondestructive Examination of PVRC Plate-Weld Specimen 251 J-Part A." Welding Research Council Bulletin 221.

Bush, S. H. 1980. "Nondestructive Examination and Component Reliability: What Should We Be Looking For." Paper presented at the Third International Conference on NDE in the Nuclear Industry, February 11-13, 1980, Salt Lake City. Sponsored by the American Society for Metals.

Chel1, G. G. 1979. "A Procedure for Incorporating Thermal and Residual Stresses Into the Concept of a Failure Assessment Diagram." In ASTM STP-668, American Society of Testing and Materials, pp. 581-605. 
Combustion Engineering, Inc. 1977. Design Basis in Pipe Breaks for the Combustion Engineering Two Loop Reactor Coolant System, CENPD-168.-A.

Eiber, R. J., M. E. Mayfield, E. C. Rodabaugh, B. N. Leis. 1979. Cold Leg Integrity Evaluation Phase I. Prepared for the U.S. Nuclear ReguTatory Commission by Battelle Columbus Laboratories.

EPRI. 1976. Proceedings of NDE Experts Workshop on Austenitic Pipe Inspection. EPRI SR-30, Electric Power Research Institute, PaTo ATto, California.

Erdogan, F., G. R. Irwin and M. Ratwani. 1976. Ductile Fracture of Cylindrical Vessels Containing a Large Flaw. ASTM-STP-601, American Society for Testing Materials.

Esselman, T. C., W. H. Bamford, E. T. Hughes and D. C. Adamonis. 1976. Augmented Inservice Inspection of Piping Welds at the Reactor Vessel Nozzles. WCAP-8802, Westinghouse Electric Copr., Nuclear Energy Systems.

Finney, 0. J. 1964. Statistical Methods and Biological Assay. Hafner Publishing Company, New York.

Folias, E. S. 1965. "An Axial Crack in a Pressurized Cylindrical Shell." Int. J. Fract. Mech. 1(2):104-113.

For 1i, 0. 1979a. "Comparison of Radiography and Ultrasonic Testing." Paper presented at the Second Nordiske NOT Symposium, May 21-23, 1979, Kobenhaum.

For li, 0. 1979b. "Comparison of Radiography and Ultrasonic Testing." Paper presented at the Second NOrdiske NDT Symposium, May 21-23, 1979, Kobenhaum.

Forli, 0. 1979c. "Reliability of Ultrasonic and Radiographic Weld Testing." Paper presented at the Ninth World Conference on Nondestructive Testing, 1979, Melbourne.

Gilman, J. D. 1968. Stress Intensity Factor for a Circumferent Through-Wall Crack in a Straight Pipe. GEAP-5557, General Electric Company.

Gruber, G., Dr. 1979. "Defect Identification and Sizing by the U1trasonic Satellite-Pulse Technique." Proceedings of ARPA-AF Review of Progress in Quant itative NOE. July 1979, La Jolla, California.

Hooper, J. L., ed. 1979. Reactor Safety Research Programs Quarterly Report. NIJREG/CR-0681, PNL-2653-4. Prepared for the U.S. Nuclear Regulatory Commission by the Pacific Northwest Laboratory, Richland, Washington.*

Kanninen, et al. 1976. Mechanical Fracture Predictions for Sensitized Stainless Steel Piping with Circumferential Cracks. EPRI-NP-192. Prepared for the Electric Power Research Institute by Battelle Columbus Laboratories. 
Kiefner, J. F., W. A. Maxey, R. J. Eiber and A. R. Duffy, 1973. "Failure Stress Levels of Flaws in Pressurized Cylinders." In ASTM STP-536, American Society of Testing and Materials, pp. 461-481.

Krautkramer, J., and H. Krautkramer. 1969. Ultrasonic Testing of Materials, Springer-Verlag, New York, NY.

Marston, T. U., ed. 1978. Flaw Evaluation Procedures: Background and Application of ASME Section XI, Appendix A. EPRI NP-719-SR, Prepared for Electric Power Research Institute by American Society of Mechanical Engineers Section XI Task Group on Flaw Evaluations.

Mayfield, M. E., T. P. Forte, E. C. Rodabaugh, B. N. Leis and R. J. Eiber. 1980. Final Report on Cold Leg Integrity Evaluation. NUREG/CR-1319. Prepared for the U.S. Nuclear Regulatory Commission by Battelle Columbus Laboratories. *

Newman, J. C., Jr. 1976. Fracture Analysis of Surface and Through Cracks in Cylindrical Pressure Vessels. NASA TN D-8325, National Aeronautics and Space Administration.

Paris, P. C., H. Tada, A. Zahoor and H. Ernst. 1979. "The Theory of Instability of the Tearing Mode of Elastic-Plastic Crack Growth." In ASTM STP-668, American Society of Testing and Materials, pp. 5-36.

PISC (Plate Inspection Steering Committee). 1979. Evaluation of the PISC Trail Results, EUR 6371, Vol. V, Commission of the European Communities, Brussels.

Reyno1ds, M. B. 1968. Failure of flawed ASTM A106B Pipes Containing Axial Through-Wal1 Flaws. GEAP-5620, General Electric Company.

Server, W. L. 1979. "Static and Dynamic Fibrous Initiation Toughness Results for Nine Pressure Vessel Materials." In ASTM STP-668, American Society for Testing and Materials, pp. 493-514.

Server, W. L., and W. 01dfield. 1978. Nuclear Pressure Vessel Steel Data Base, EPRI NP-933. Prepared by Fracture Control Corporation, Golita, California for Electric Power Research Institute.

Serabian, S., and W. E. Lawrie. 1977. "A Detection Model for Pulse Echo Ultrasonics." Paper presented at the ASNT Fall Meeting, September 1977.

Silk, M. G. 1978. Estimates of the Magnitude of Some of the Basic Sources of Frror in Ultrasonic Defect Sizing. AERE-R-9023.

Tada, H., P. C. Paris, M. P. Gomez and R. Gamble. 1979. "A Stability Analysis of Circumferential Cracks for Reactor Piping System." Paper presented at ASTM 12th National Symposium on Fracture Mechanics, May 21-23, 1979, Washington University, St. Louis. 
Wilson, A. D. 1979. "Chracterization of Plate Steel Quality Using Various Toughness Measurement Techniques." In ASTM STP-668, American Society for Testing and Materials, pp. 469-492.

*Available for purchase from the NRC/GPO Sales Program, U.S. Nuclear Regulatory Commission, Washington, DC 20555, and the National Technical Information Service, Springfield, VA 22161. 


\section{APPENDIX A}

DEFECT SENSITIVITY STUDY 


\section{APPENOIX A \\ DEFECT SENSITIVITY STUDY}

\section{PURPOSE AND SCOPE}

The purpose of the study reported here was to calculate crack tip stress instensity factors for a set of representative defects of interest to reactor pressure vessel and piping integrity. It was thought that only through the calculation of actual numerical values of stress intensity factors could one obtain a "feel" for the absolute and relative significance of specific types of defects. The following specific factors were considered:

- Crack size relative to vessel thickness (i.e., $0.205,0.10,0.25$, 0.50 , and 0.75 of wall).

- Stress level normal to crack plane $(10,20,40$ and $60 \mathrm{ksi})$.

- Crack aspect ratio with circular and elliptical (10:1) cracks considered.

- Surface cracks versus cracks embedded in the wall of the vesse?.

- Location of embedded defect relative to the surfaces of vessel.

- Crack at inner diameter of vessel versus crack at outer surface of vessel.

- Crack normal to maximum tensile stress versus cracks at arbitrary angles to this tensile stress.

- Level of axial stress in a vessel relative to hoop stress $(0.5,1.0$, 2.0 and 3.0 times hoop stress).

- Stress gradient through the wall of the vessel such as produced by bending moments, thermal stresses, or residual stresses.

\section{REFERENCE GEOMETRY AND LOAOINGS}

Figure A.1 shows the specific vessel or pipe geometry which was the focus of this study. In most of the calculations the curved geometry was approximated 
by the flat plate geometry of Figure A.1b. The following parameters were selected:

$$
\begin{aligned}
& \text { Wall thickness }=4.00,8.00 \text {, and } 12.00 \mathrm{in} . \\
& \text { Hoop stress }=20 \mathrm{ksi} \\
& \text { Axial stress }=20 \text { to } 60 \mathrm{ksi} \\
& \text { Implied material yield strength }=60 \mathrm{ksi} \\
& \text { Fracture toughness }=\text { unspecified }
\end{aligned}
$$

The implied material yield strength of $60 \mathrm{ksi}$ was taken to be typical of a material used for reactor vessels and piping. The stress levels were intended to be consistent with ASME code allowable levels. At $20 \mathrm{ksi}$ the hoop stress corresponds to the primary membrane stress limitation of the code. Axial stresses up to $60 \mathrm{ksi}$ include secondary stresses from such sources as thermal gradients or piping restraints.

While specific dimensions and stress levels were selected to give physically meaningful numbers for stress intensity factors, the results can be readily scaled to apply the results to other situations. Specifically, crack tip stress intensity factors are proportional to the applied stress level. Futhermore, the usual fracture mechanics relationship holds, in that stress intensities are proportional to the square root of characteristic dimensions. For example, for a wall thickness of $2.0 \mathrm{in.}$ (as opposed to a thickness of 4.0 in.), all stress intensity factors (for a fixed ratio of crack dimension to wall thickness) can be proportioned by the factor $\sqrt{1 / 2}=0.707$.

\section{STRESS INTENSITY FACTOR SOLUTIONS}

Stress intensity factors were computed from published solutions and the. literature was surveyed to locate "definitive" solutions for the configurations of interest. To some extent the specific crack geometries were selected to match available published results.

For surface cracks of elliptical and semicircular shape, the finite element results of Newman and Raju (1977) were used. Subsurface cracks of the same type were analyzed on the bas is of solutions by Kobayashi, Ziv, and Hall (1965) for centrally positioned defects. Kobayashi, Ziv and Hall also 


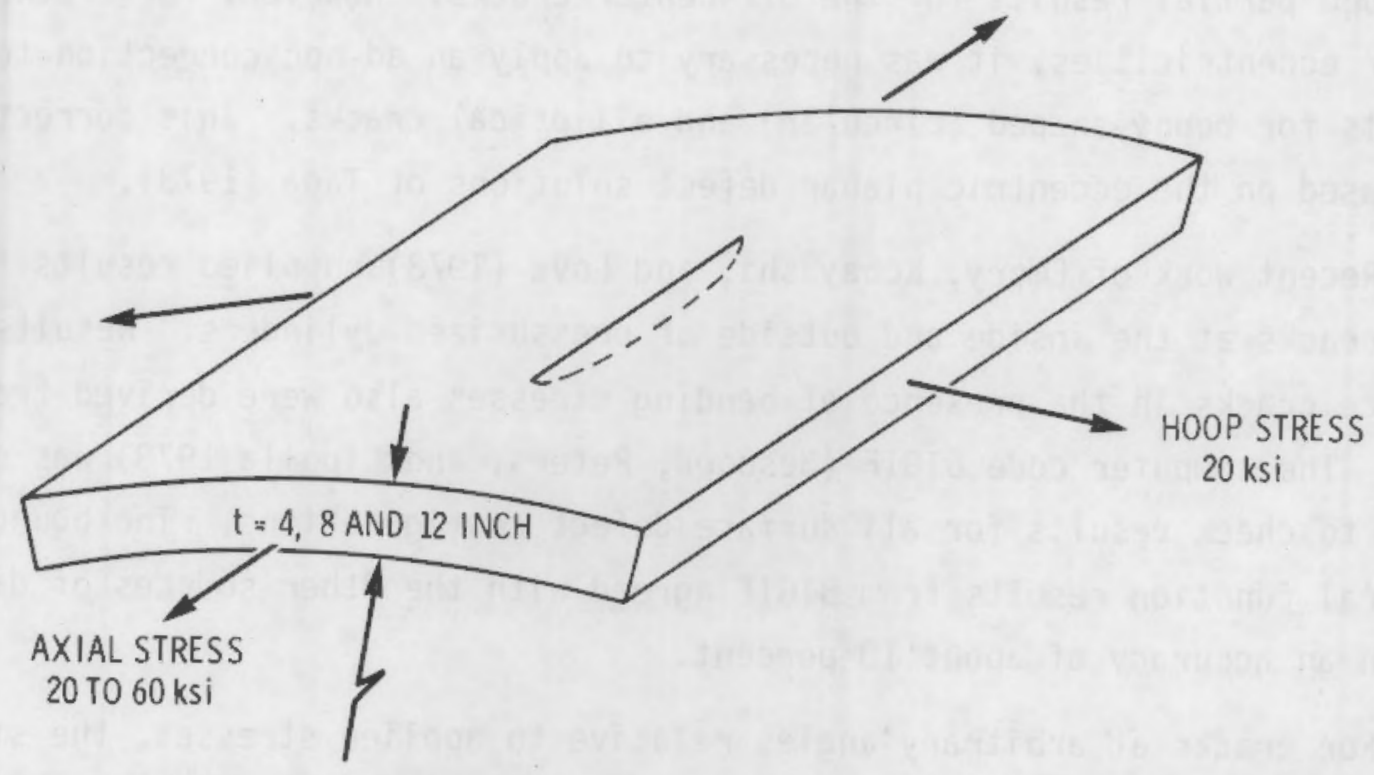

(a) REFERENCE GEOMETRY

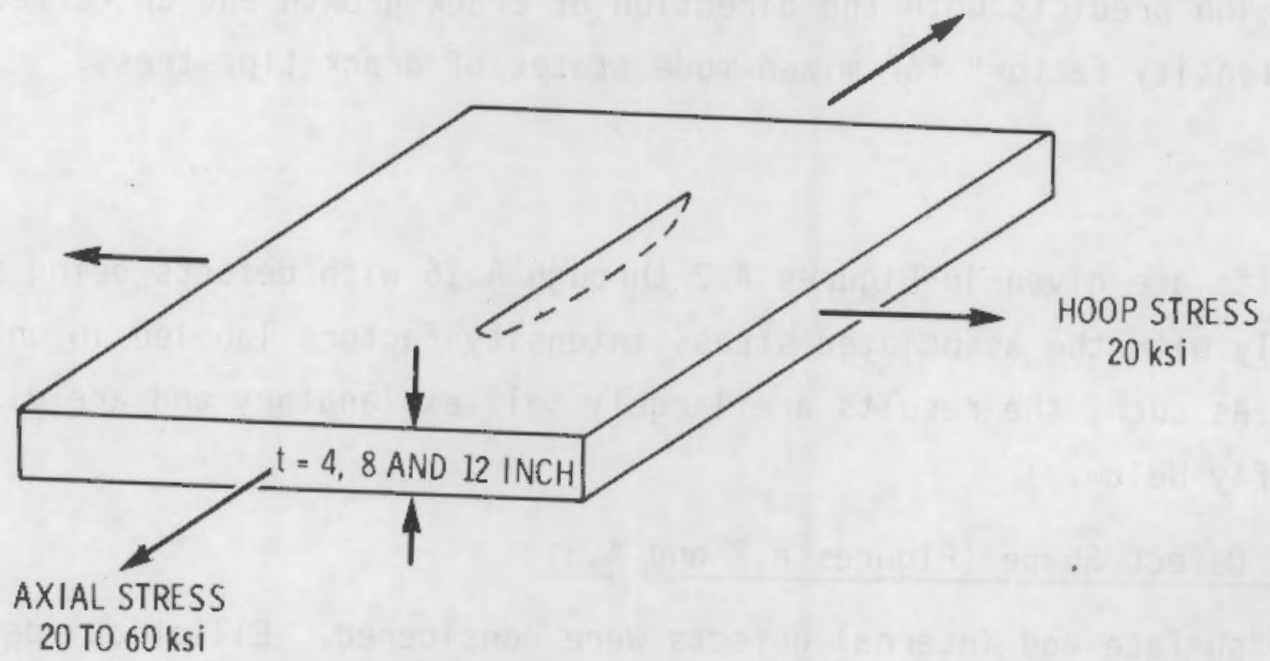

(b) FLAT PLATE APPROXIMATION

FIGURE A.1. Reference Geometry and Loadings 
provided partial results for the off-center cracks. However, for cracks with larger eccentricities, it was necessary to apply an ad-hoc correction to results for penny-shaped (circular) and elliptical cracks. This correction was based on the eccentric planar defect solutions of Tada (1973).

Recent work of Emery, Kobayashi, and Love (1978) supplied results for surface cracks at the inside and outside of pressurized cylinders. Results for surface cracks in the presence of bending stresses also were derived from their work. The computer code BIGIF (Besuner, Peters, and Cipolla 1978) was exercised to check results for all surface defect configurations. The boundary integral function results from BIGIF agreed with the other sources of data within an accuracy of about 10 percent.

For cracks at arbitrary angles relative to applied stresses, the strain energy density criterion of crack growth as proposed by $\sin$ (1973) was applied. The criterion predicts both the direction of crack growth and an "effective stress intensity factor" for mixed-mode states of crack tip stress.

\section{RESULTS}

Results are given in Figures A.2 through A.16 with defects being shown pictorially with the associated stress intensity factors labeled in units of ksi $\sqrt{i n}$. As such, the results are largely self-explanatory and are discussed only briefly below.

\section{Effect of Defect Shape (Figures A.2 and A.3)}

Both surface and internal defects were considered. Elliptical defects result in higher stress intensity factors than the penny-shaped (circular) defects. For the large defects, the elliptical defects are nearly twice as severe as the circular defects of the same depth.

Effect of Defect Location (Figures A.4 and A.5)

Surface defects through a given fraction of the section thickness are compared with the corresponding internal defects located at midsection. Surface defects are seen to be somewhat more severe than internal defects. 


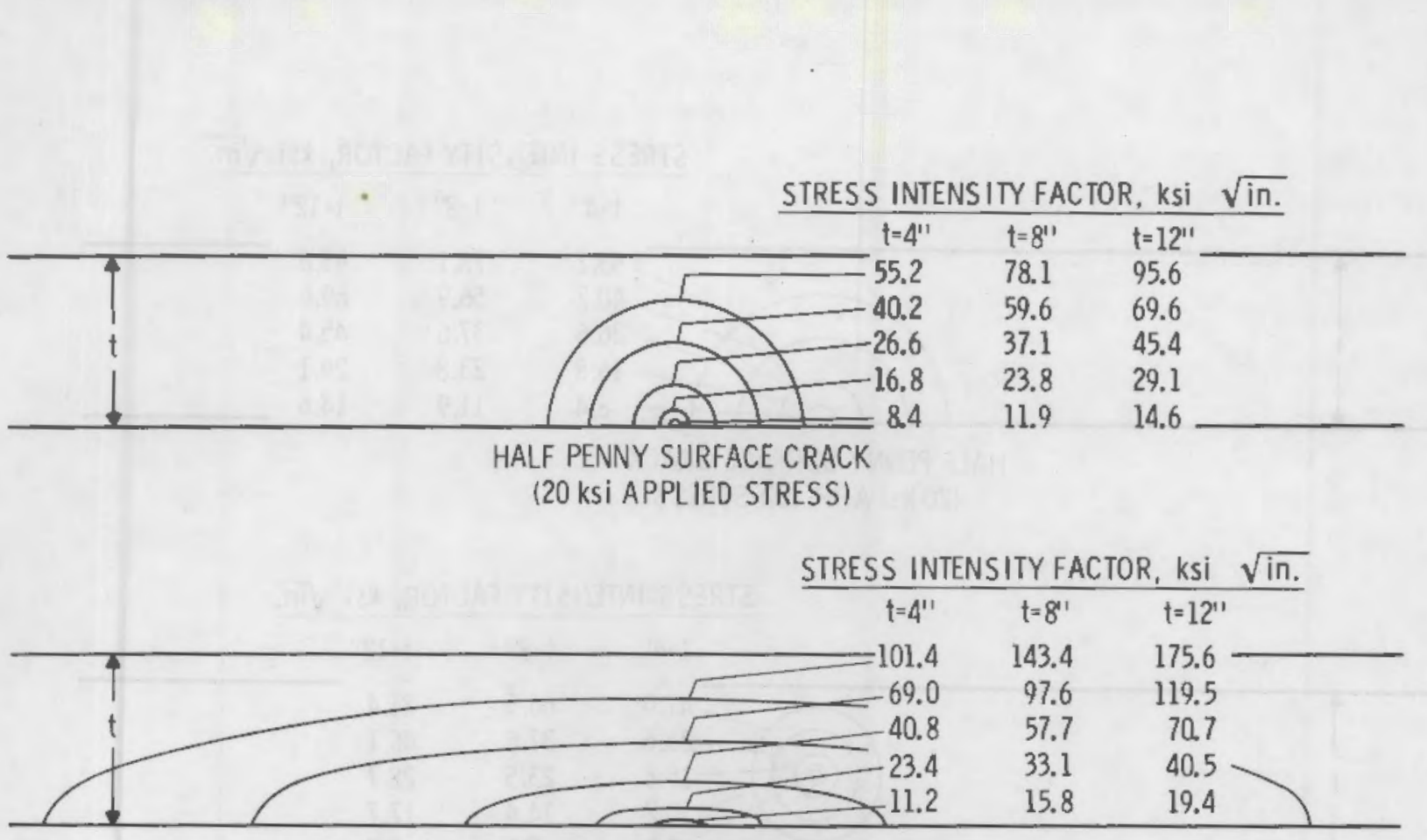

ELLIPTICAL SURFACE CRACK (10:1)

(20 ksi APPLIED STRESS)

FIGURE A.2. Effect of Defect Shape - Surface Half-Penny Versus Surface Elliptical Crack

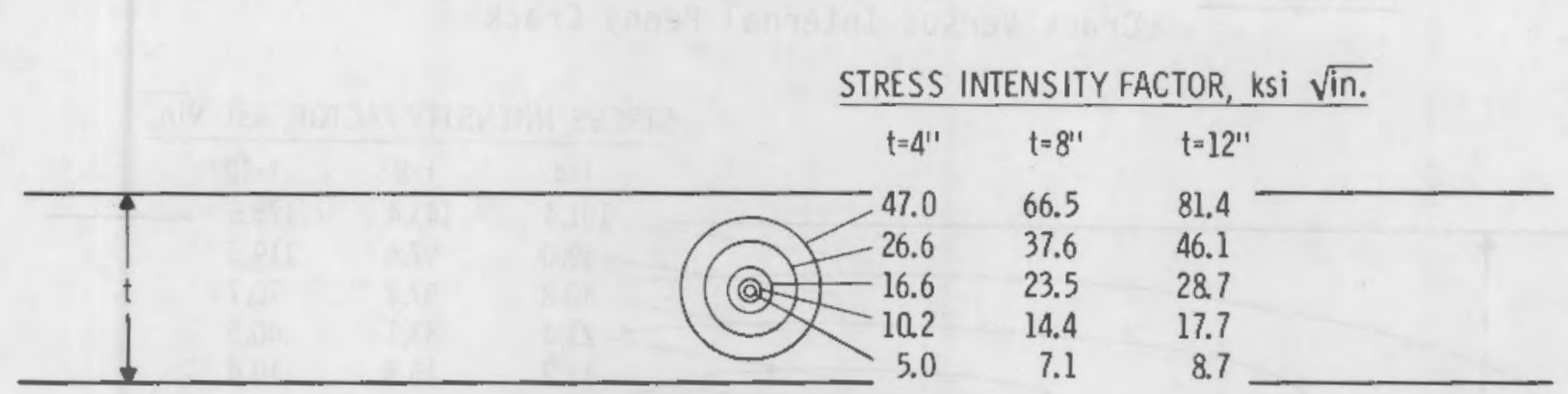

PENNY SHAPED INTERNAL CRACK

(20 ksi APPLIED STRESS)

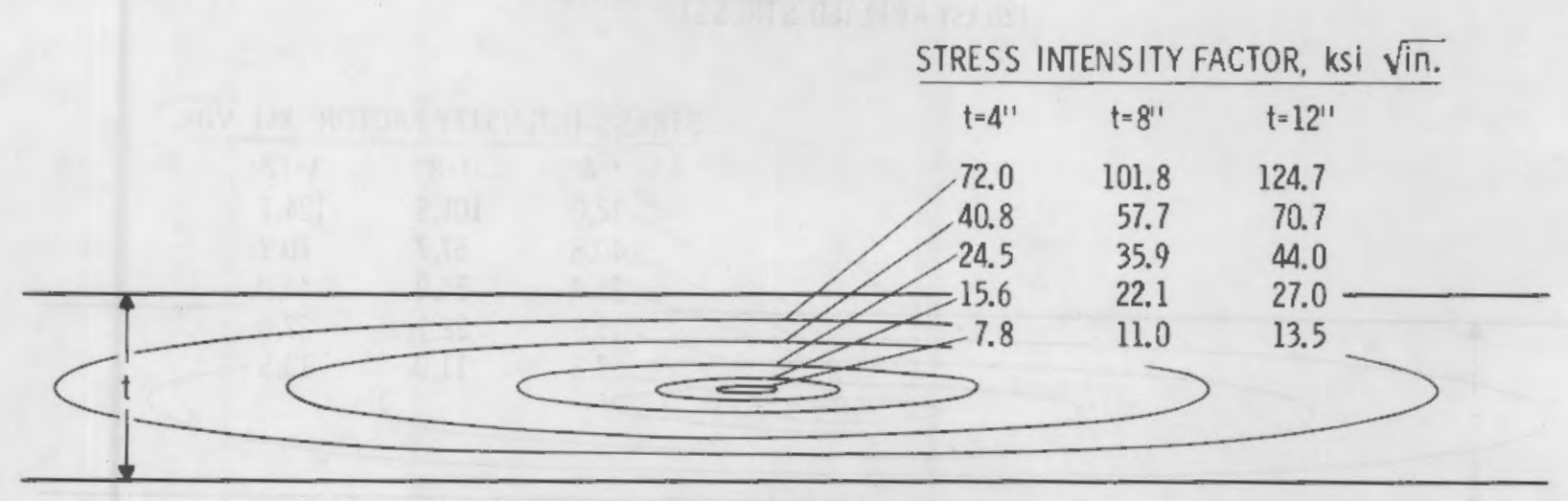

ELLIPTICAL INTERNAL CRACK $(10: 1)$

(20 ksi APPLIED STRESS)

FIGURE A.3. Effect of Defect Shape - Internal Penny Versus Internal Elliptical Crack 
STRESS INTENSITY FACTOR, ksi $\sqrt{\mathrm{in}}$.

$t=4^{\prime \prime} \quad t=8^{\prime \prime} \quad t=12^{\prime \prime}$

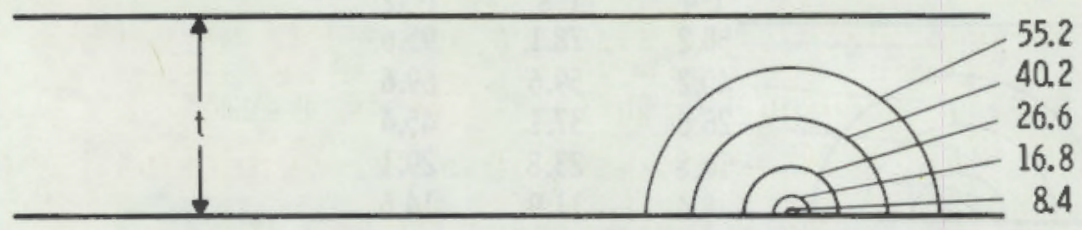

$78.1 \quad 95.6$

$56.9 \quad 69.6$

$37.6 \quad 45.4$

$23.8 \quad 29.1$

$11.9 \quad 14.6$

HALF PENNY SURFACE CRACK

(20 ksi APPLIED STRESS)

STRESS INTENSITY FACTOR, ksi $\sqrt{\mathrm{in} .}$

$t=4^{\prime \prime} \quad t=8^{\prime \prime} \quad t=12^{\prime \prime}$

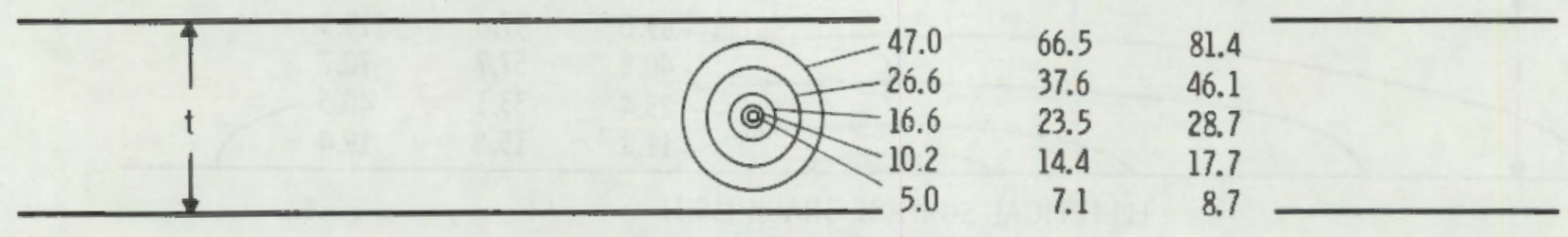

PENNY SHAPED INTERNAL CRACK

(20 ksi APPLIED STRESS)

FIGURE A.4. Effect of Defect Location - Surface Half-Penny Crack Versus Internal Penny Crack

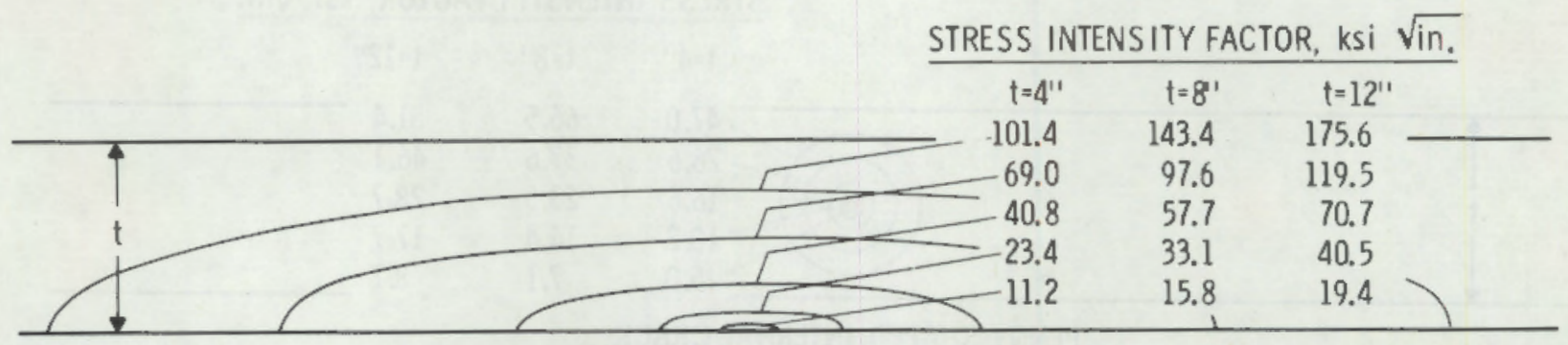

ELLIPTICAL SURFACE CRACK (10:1)

(20 ksi APPLIED STRESS)

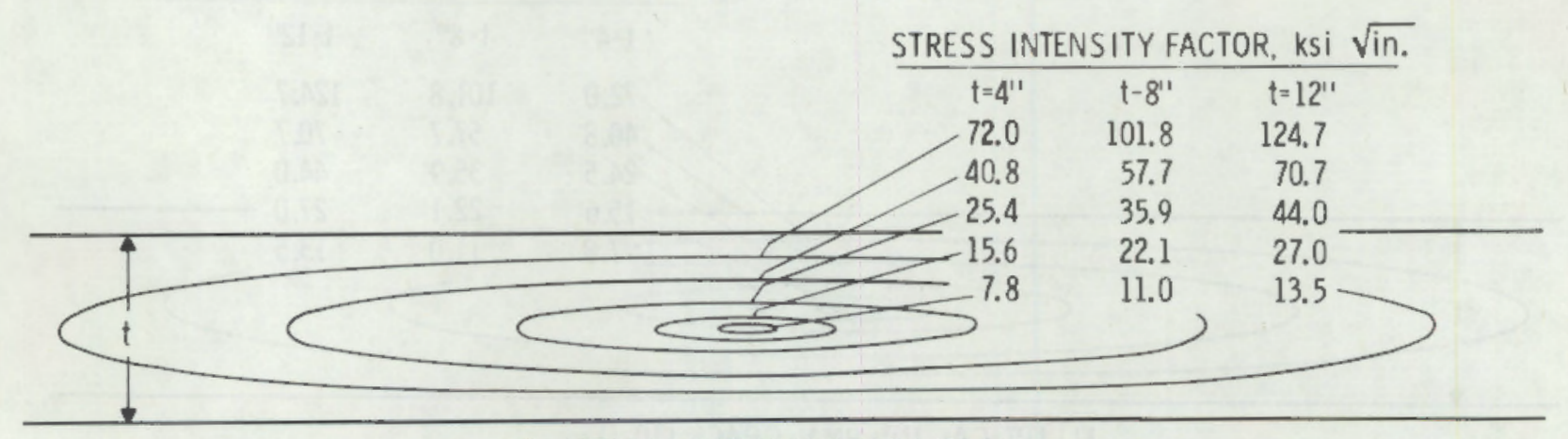

ELLIPTICAL INTERNAL CRACK (10-1)

(20 ksi APPL IED STRESS)

FIGURE A.5. Effect of Defect Location - Surface Elliptical Crack Versus Internal Elliptical Crack 
Effect of Surface Proximity (Figure A.6 and A.7)

Circular and elliptical defects of increasing size are assumed to be located at midsection at locations approaching the surface. It is assumed that the applied stress is uniform through the thickness of the cross section. It is seen that the stress intensities are relatively insensitive to the location of the defect.

Effect of Defect Orientation (Figures A.8 through A.11)

In this study the hoop stress was fixed at $20 \mathrm{ksi}$ and the axial stress was increased from $10 \mathrm{ksi}$ to $60 \mathrm{ksi}$. The calculations were for 1-in.-long planar through-wall cracks. The dashed lines indicate the predicted direction of crack propagation. In extending, the cracks tend to turn and align with the direction of maximum tensile stress. In all cases the most severe crack is normal to the direction of maximum tensile stress. Also, cracks not al igned to the directions of the principal stresses are of intermediate severity to cracks aligned with the imposed hoop and axial stresses. Futhermore, the variation of stress intensities with angle shows only a modest deviation from a linear trend.
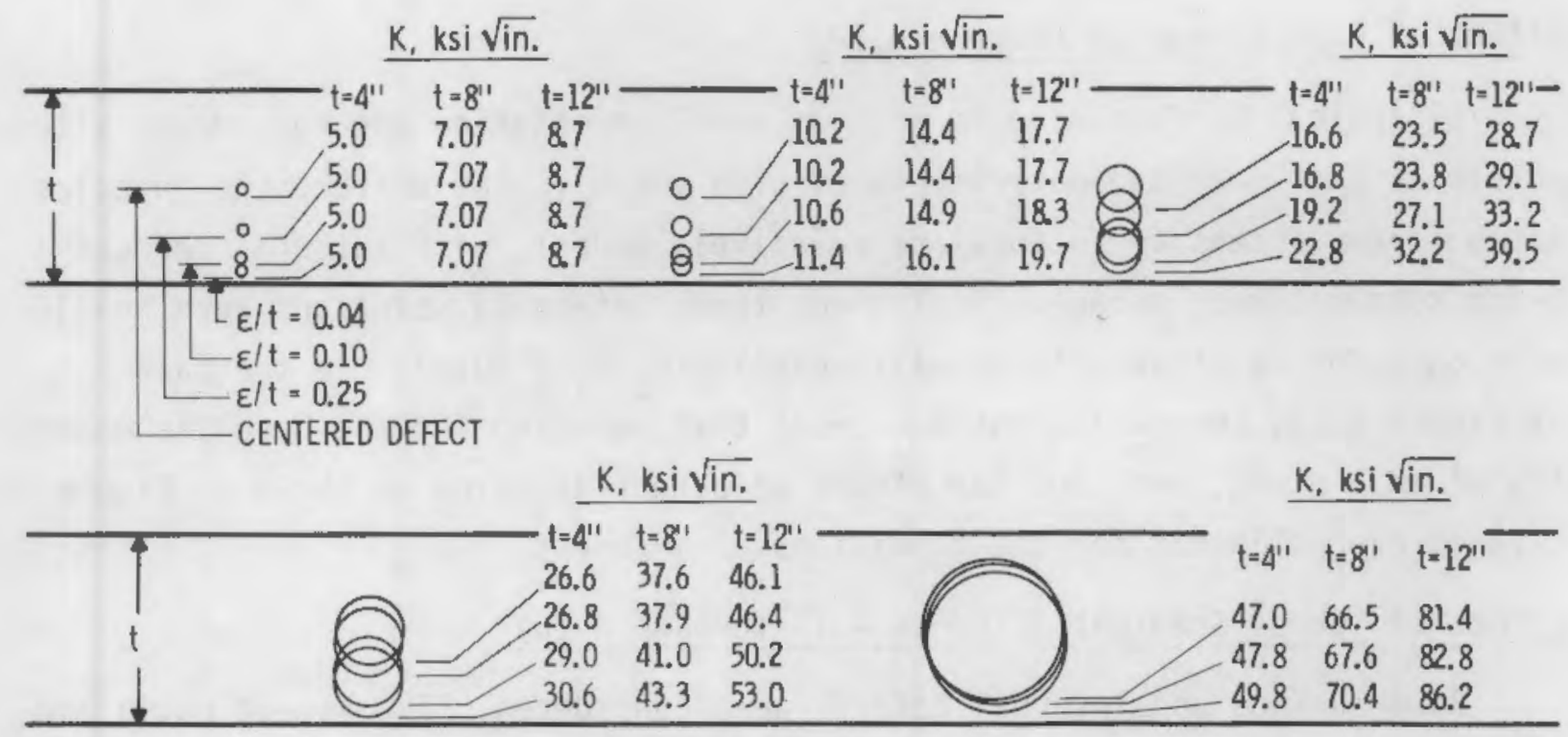

PENNY SHAPED INTERNAL CRACKS

(20 ksi APPLIED STRESS)

FIGURE A.6. Effect of Surface Proximity - Internal Penny Crack 

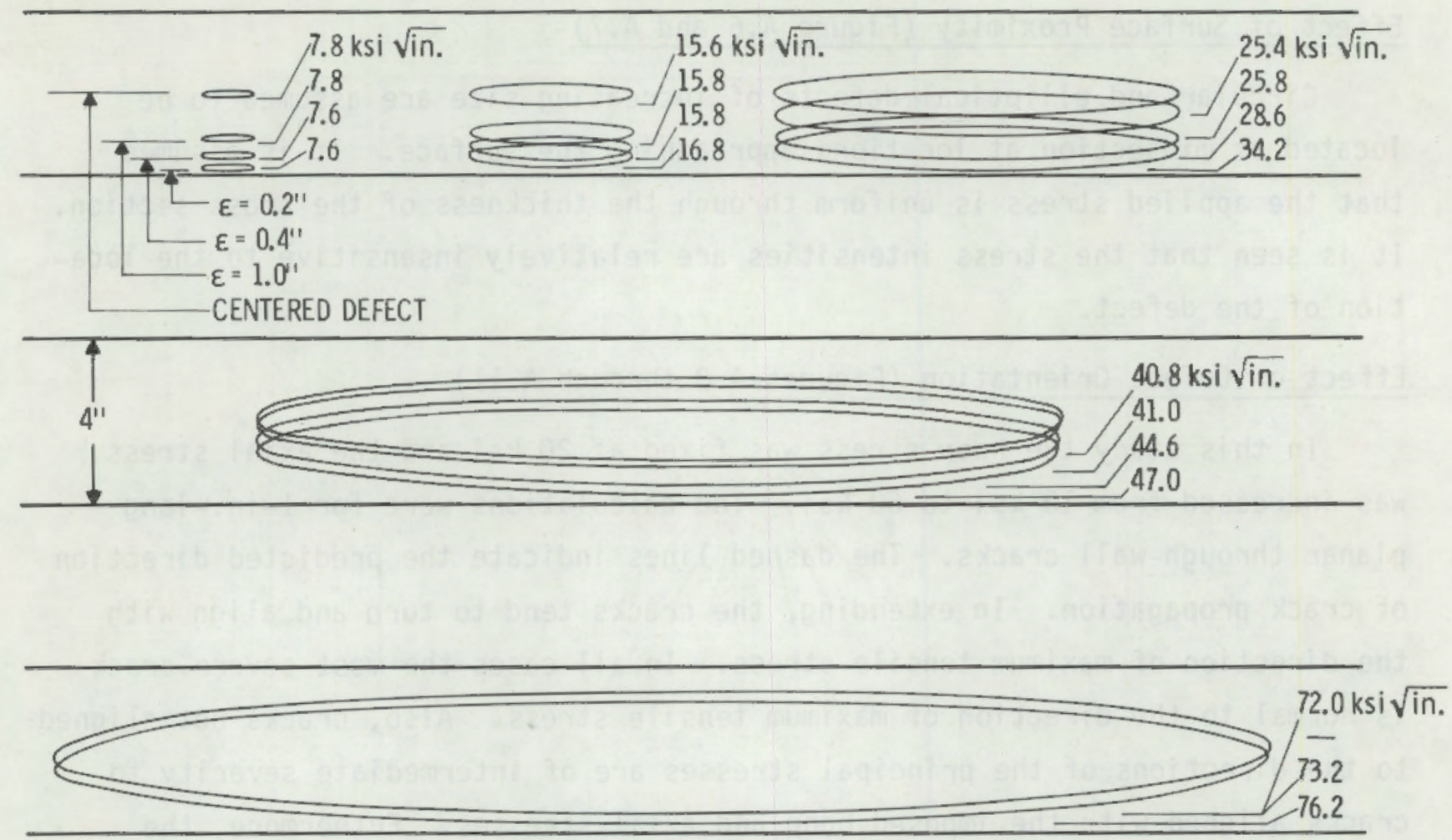

ELLIPTICAL INTERNAL CRACKS (10:1)

(20 ksi APPLIED STRESS)

FIGURE A.7. Effect of Surface Proximity - Internal Elliptical Crack

Effect of Pipe Curvature (Figure A.12)

Elliptical surface defects with an axial orientation are considered alternately at the inner and outer surfaces of a vessel. The differences in calculated stress intensity factors are relatively modest, with internal defects being somewhat more severe. Published stress intensity solutions were available only for relatively thick wall vessels $\left(R_{0} / R_{i} \geq 10 / 9\right)$. In the case of Figure A.12, the radius ratio exceeds that selected for the baseline geometry of this study, and thus the effect of defect location as shown in Figure A.12 is overestimated for the conditions of interest. Effect of Stress Gradient (Figures A.13 through A.16)

Both surface and internal defects were considered. The aspect ratio and depth of the flaws were varied.

Figures A.13 and A.14 show results for half-penny (semi-circular) and elliptical (10:1 aspect ratio) surface cracks of various depths into the 


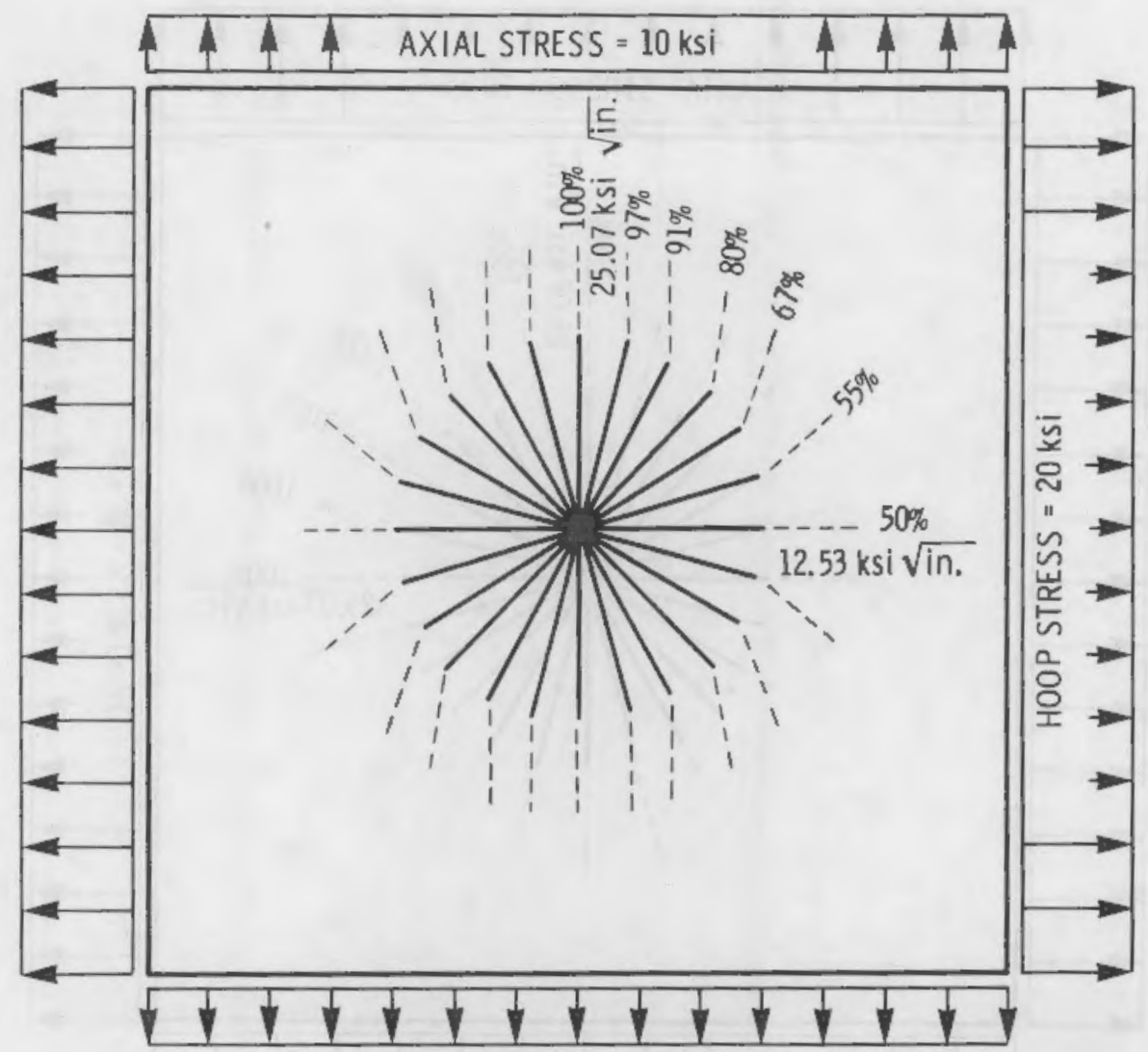

FIGURE A.8. Effect of Defect Orientation (10-ksi Stress Combined with 20-ksi Hoop Stress)

wal1. The stress distribution through the wall was varied from pure tension to pure bending. In each case the maximum surface tension at the defect location had the fixed level of $60 \mathrm{ksi}$. Thus, the surface defect extended into regions of decreasing tensile stress and in some instances into regions of compressive stress. For small surface defects, the nature of the stress distribution (pure tension or bending) was relatively insignificant. For relatively deep defects (extending through half or more of the cross section), pure tension is notably more severe than pure bending.

Figures A.15 and A.16 show results for internal penny-shaped and elliptical (10:1 aspect ratio) cracks. The stress distribution for the surface defects was varied from pure tension to pure bending. In all cases the internal defects were substantially less severe than the corresponding surface defects. 


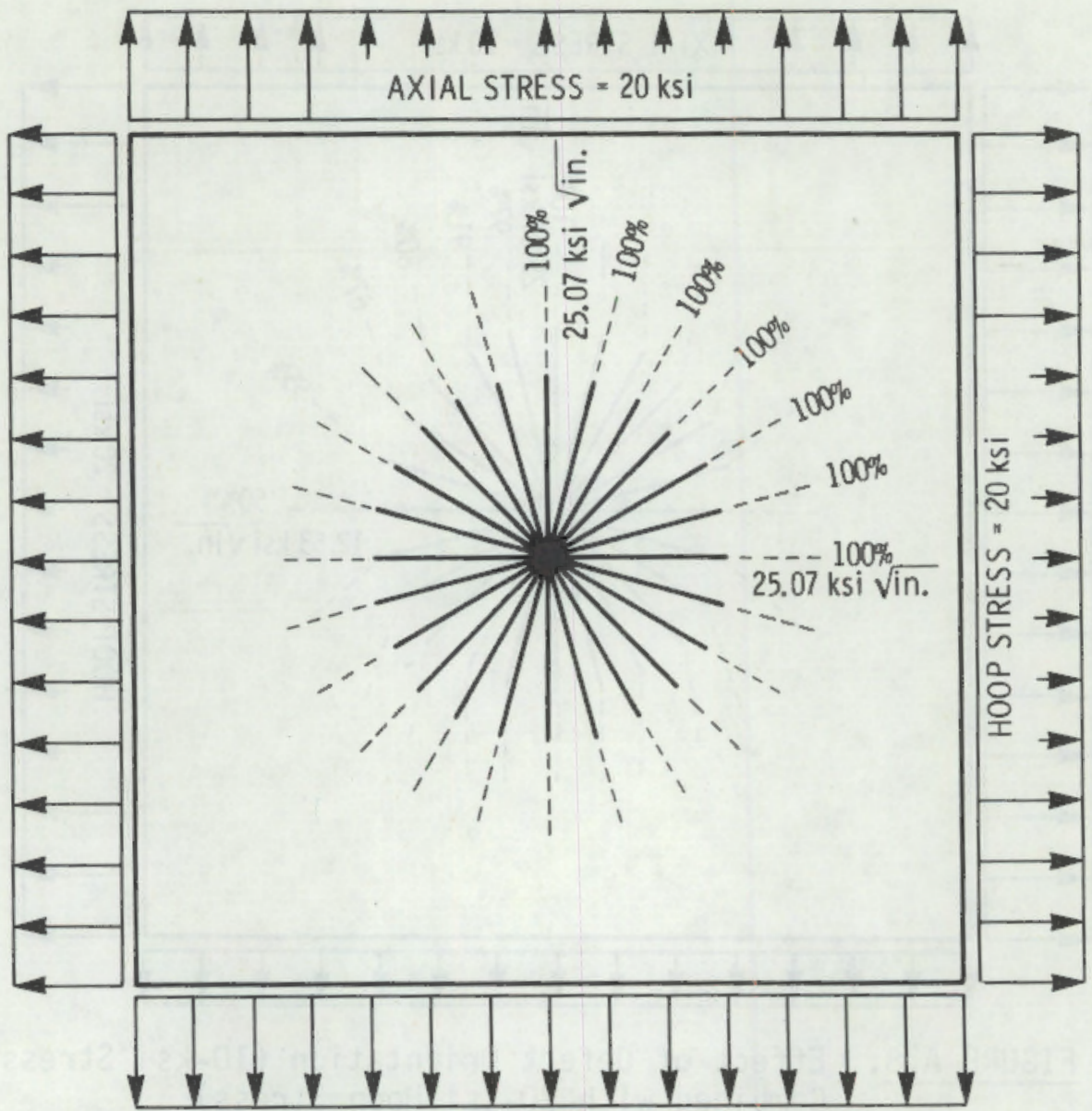

FIGURE A.9. Effect of Defect Orientation (20-ksi Axial Stress Combined with 20-ksi Hoop Stress) 


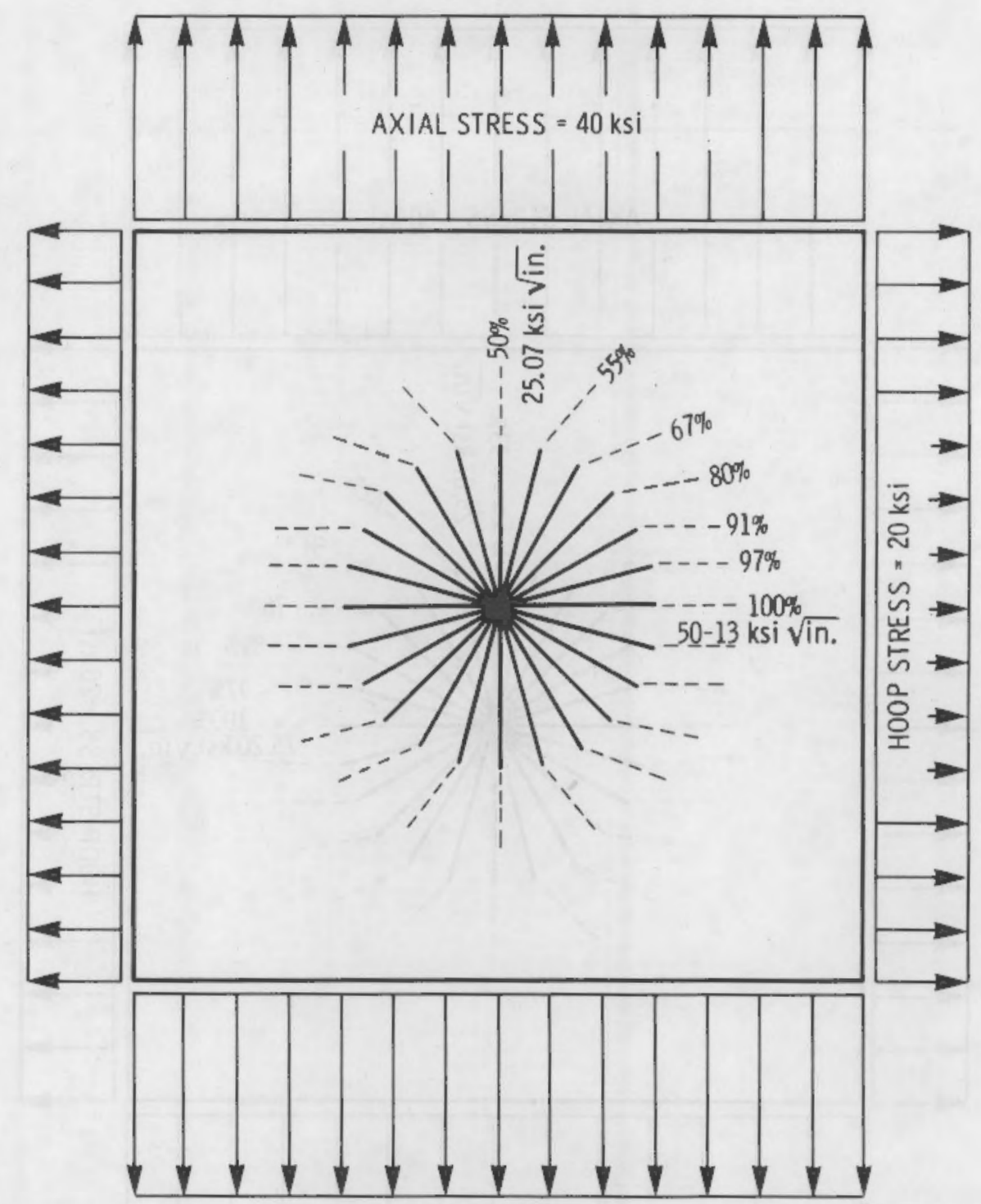

FIGURE A.10. Effect of Defect Orientation (40-ksi Axial Stress Combined with 20-ksi Hoop Stress) 




FIGURE A.11. Effect of Defect Orientation (60-ksi Axial Stress Combined with 20-ksi Hoop Stress) 

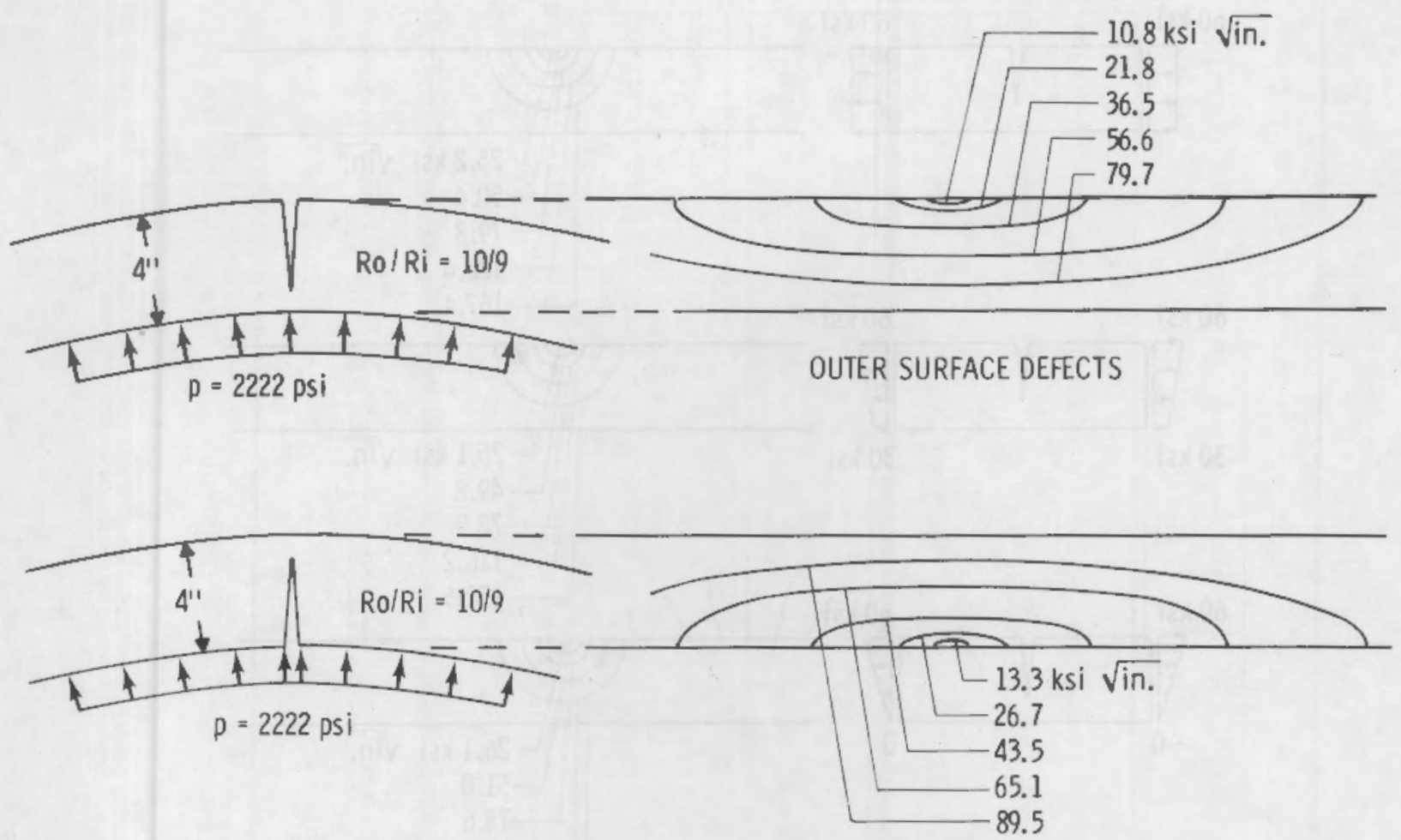

INNER SURFACE DEFECTS

FIGURE A.12. Effect of Pipe Curvature - External Versus Internal Elliptical (10:1) Surface Crack 


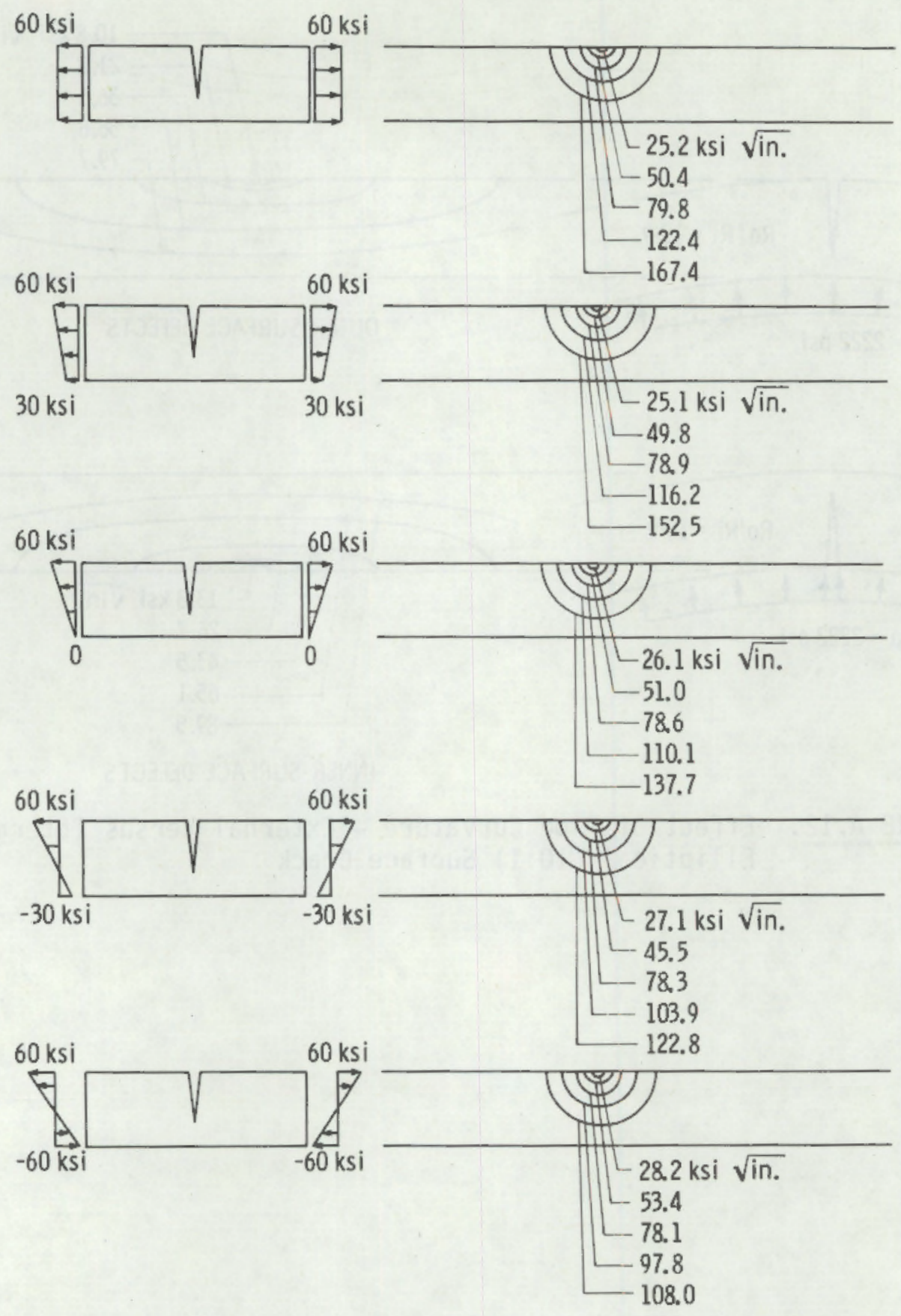

FIGURE A.13. Effect of Through-Wall Stress Gradient Half-Penny Surface Crack 


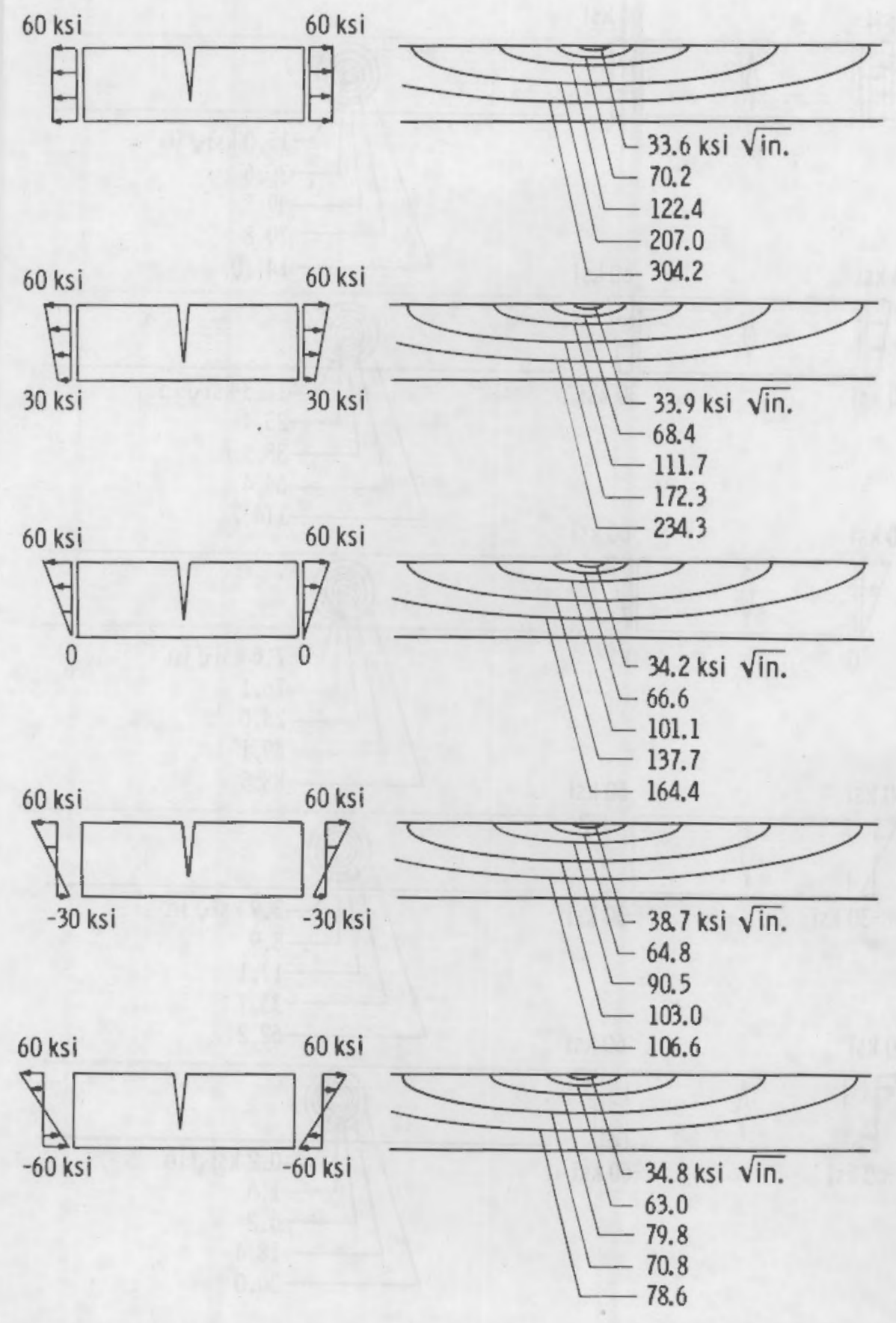

FIGURE A.14. Effect of Through-Wall Stress Gradient Elliptical (10:1) Surface Crack 


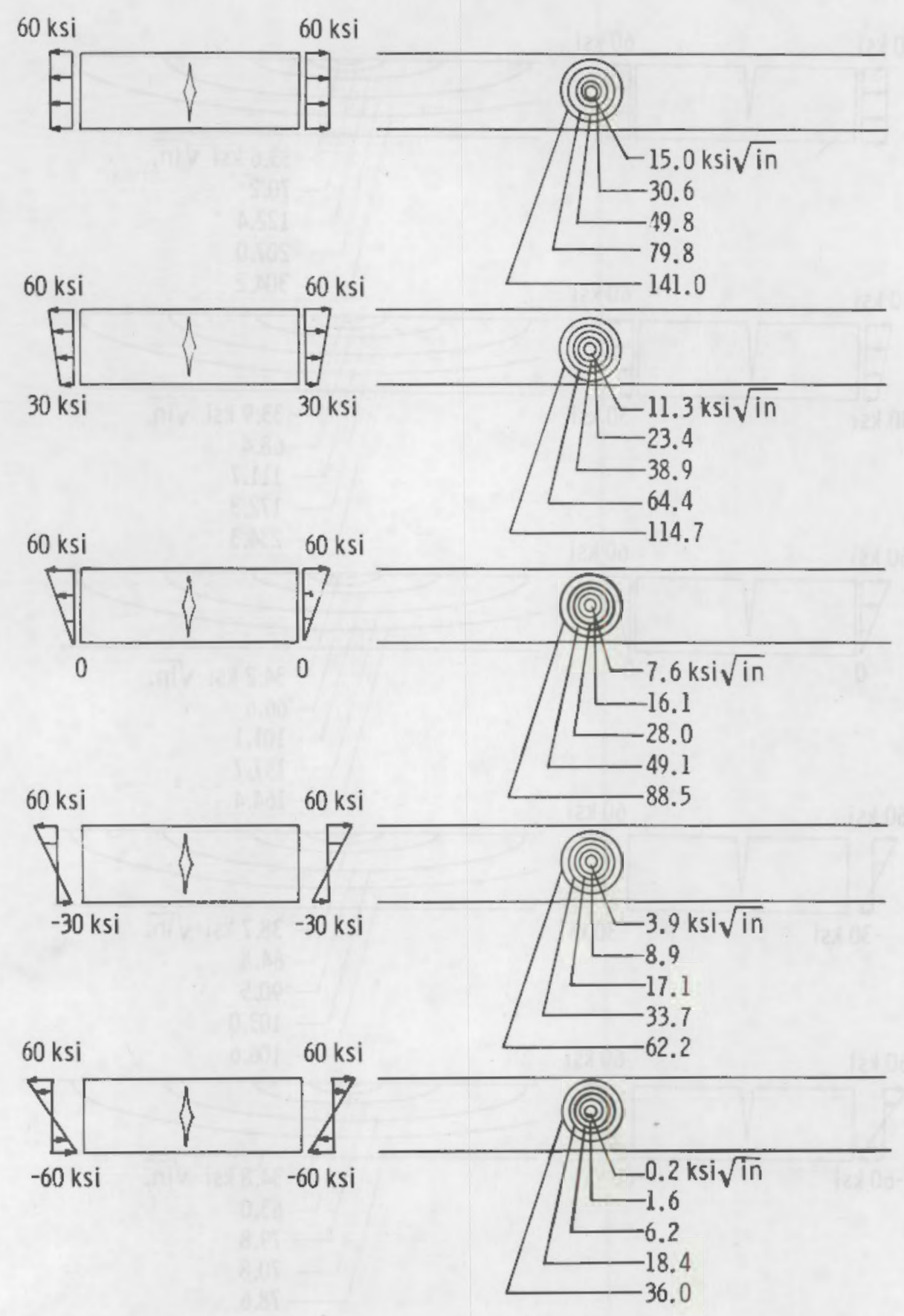

FIGURE A.15. Effect of Through-Wall Stress Gradient Internal Penny Crack 


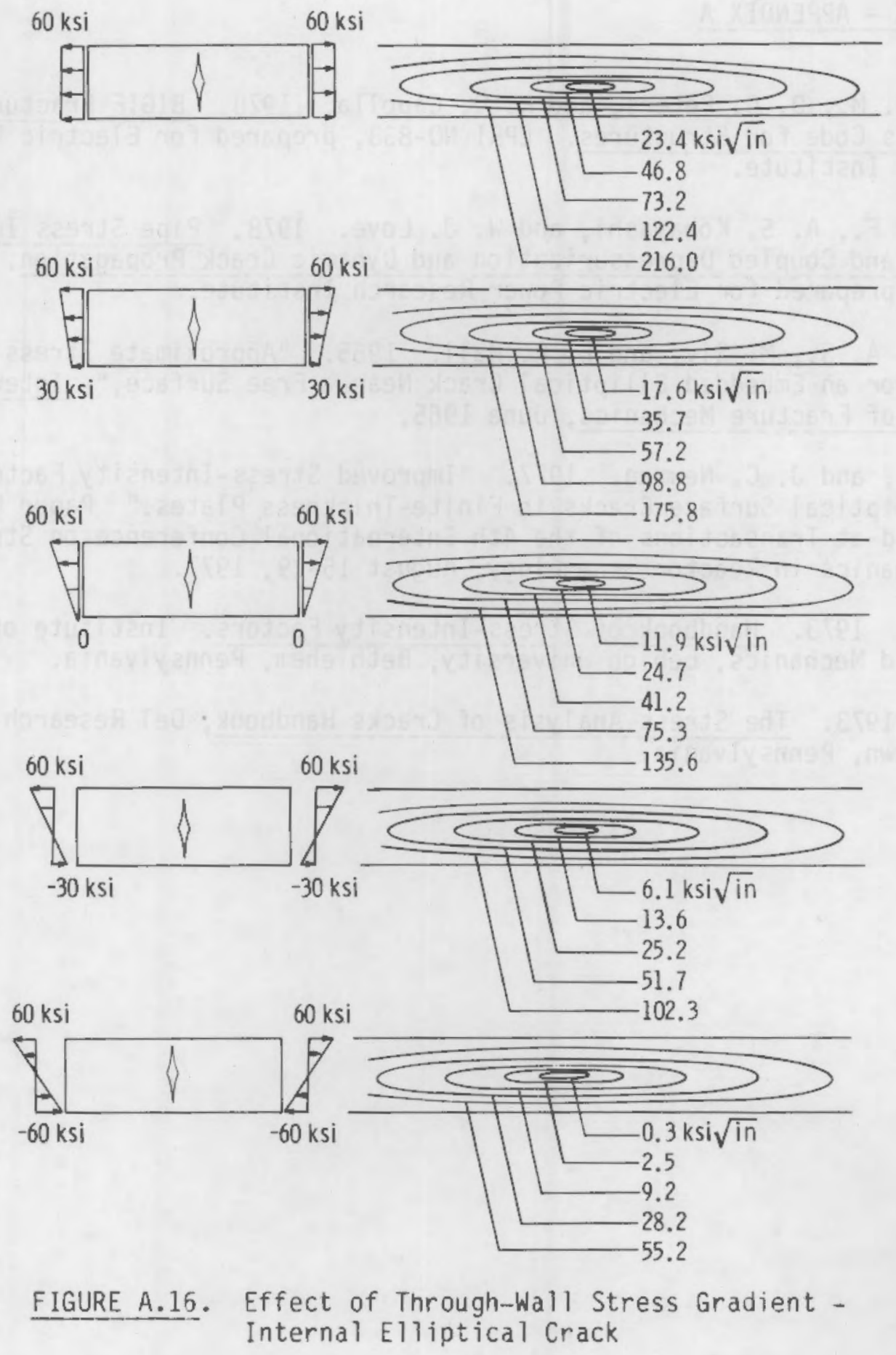


Besuner, P. M., D. C. Peters, and R. C. Cipolla. 1978 BIGIF Fracture Mechanics Code for Structures. EPRI N0-838, prepared for Electric Power Research Institute.

Emergy, A. F., A. S. Kobayashi, and W. J. Love. 1978. Pipe Stress Intensity Factors and Coupled Depressurization and Dynamic Crack Propagation. EPRI NP-763, prepared for Electric Power Research Institute.

Kobayashi, A. S., M. Siv, and L. R. Hall. 1965. "Approximate Stress Intensity Factor for an Embedded Elliptical Crack Near a Free Surface," International Journal of Fracture Mechanics, June 1965.

Raju, I.S., and J. C. Newman. 1977. "Improved Stress-Intensity Factors for Semi-Elliptical Surface Cracks in Finite-Thickness Plates." Paper No. G5/8 presented at Transactions of the 4th International Conference on Structures and Mechanics in Reactor Te hnology, August 15-19, 1977.

Sih, G. C. 1973. Handbook of Stress-Intensity Factors. Institute of Fracture and Solid Mechanics, Lehigh Jniversity, Bethtehem, Pennsylvania.

Tada, H. 1973. The Stress Analysis of Cracks Handbook, Del Research Corps., Hellertown, Pennsylvania. 
APPENDIX B

THERMAL, RESIDUAL, AND PIPING FLEXIBILITY STRESSES 


\section{APPENDIX B \\ THERMAL, RESIDUAL, ANO PIPING FLEXIBILITY STRESSES}

In estimating critical flaw sizes both internal pressure loads and the effects of thermal, residual and piping flexibility stresses were considered. This Appendix describes how the level and effect of the latter types of loads were estimated.

THERMAL AND RESIOUAL STRESSES

An approximate method to account for residual stresses and thermal stresses due to through-wall thermal gradients is described by Reed, Henry, and Kasen (1979). It is assumed that an initial crack opening displacement (COD) exists that is equal to the displacement caused by the yield strain acting over a gage length equal to the pipe thickness. Thus,

$$
\delta_{0}=\left(\sigma_{y} / E\right) t
$$

The following relationships exist between the C00, fracture toughness $K_{I C}$, and the J-integral value for initiation of crack propagation

$$
\begin{aligned}
& \delta=J_{I C} / m \sigma_{y}, \quad m \cong 1.6 \\
& K_{I C}^{2}=E J_{I C} /\left(1-v^{2}\right)
\end{aligned}
$$

The apparent reduction in fracture toughness due to thermal and residual stress can then be estimated as

$$
\Delta K_{I C}=\sigma_{y}\left[m t /\left(1-v^{2}\right)\right]^{1 / 2}
$$

This equation was applied to the piping materials listed in Table 4.4 of the main text. Table B.1 lists reductions in apparent fracture toughness due to 
TABLE B.1. Reduced Values of Fracture Toughness Due to Thermal and Residual Stresses

\begin{tabular}{llcc} 
& \multicolumn{2}{c}{$\begin{array}{c}K_{I C} \\
\text { Nominal Value }\end{array}$} & $\begin{array}{c}\text { in. } \\
\text { Reduced Value }\end{array}$ \\
SA-516 Gr. 70 & 250 & 188 \\
SA-106 Gr. 106 & 250 & 185 \\
SA-376 Gr. 304 & 250 & 212 \\
SA-351 Gr. CF8A & 250 & 208
\end{tabular}

residual stresses and thermal stress caused by through-wall temperature gradients. These reductions are relatively modest, which indicates that such residual and thermal stresses are of secondary importance in comparison to primary stresses due to internal pressure.

\section{PIPING FLEXIBILITY STRESS EQUATION}

A second source of piping thermal stresses is the expansion of the piping/ reactor system during heatup to operating temperatures. Stresses due to uniform thermal expansion should be relatively low because movement is allowed for in the design of the system. However, differential thermal expansions can occur because of different temperatures within the coolant loops. The level of such stresses are estimated with the simplified model described here. A finite-element model for piping flexibility stresses is described in the next section. The purpose of these calculations was to estimate representative levels of bending moments and axial loads. Stresses from these loads were then used to estimate critical sizes of circumferential flaws.

A greatly simplified model for piping expansion and flexibility analys is is shown in Figure B.1. Bending moments and axial loads are calculated for a temperature difference $\Delta T$ between the hot and cold legs of the simplified model. The force $P_{0}$ is the axial load in the hot leg. Consideration of bending and axial extension of members of the system results in the following solution for $P_{0}$ :

$$
P_{0}=\frac{E a \Delta T L_{0}}{L_{0} / A_{0}+L / A \cos ^{2} \theta+L^{3} \sin ^{2} \theta / 12 I}
$$


FIGURE B.1. Simplified Mode1 for Piping Expansion and Flexibility Analys is

where

$\alpha$ is the thermal expansion coefficient

$E$ is the elastic modulus

$L_{0}, A_{0}$ are the length and cross sectional areas of hot leg

$L, A, I$ are the length, cross sectional areas, and moment of inertia of cold leg.

The force and bending moment in the cold leg members are

$$
\begin{aligned}
& P=1 / 2 P_{0} \cos \theta \\
& M=1 / 4 P_{0} L \sin \theta
\end{aligned}
$$

Representative loads and stresses were calculated using the parameters of the CE design of Table 4.4 of the main text, with a 42-in. I0 $x 3.75-i n$. wall hot leg and a 30-in. ID $\times 2.5-i n$. wall cold leg. The temperature difference was taken to be $100^{\circ} \mathrm{F}$, and the modulus and expansion coefficient were $28 \times$ $10^{6} \mathrm{psi}$ and $6.6 \times 10^{-6} \mathrm{in.} / \mathrm{in.} /^{\circ} \mathrm{F}$, respectively. The following loads and stresses were calculated.

$$
\begin{array}{lrr} 
& \text { Hot Leg } & \text { Cold Leg } \\
\cline { 2 - 3 } \text { Axial Load, lb } & 941,000 & 420,700 \\
\text { Stress From Axial Load, psi } & 1,745 & 1,550 \\
\text { Bending Moment, in.-lb } & 0 & 33.9 \times 10^{6} \\
\text { Bending Stress, psi } & 0 & 17,500
\end{array}
$$


These stress levels were used in evaluations of critical flaw sizes to account for piping flexibility effects.

It is seen that stresses from axial loads are relatively low compared to stresses from internal pressure. However, estimated stresses from bending moments are substantial for the parameters of the primary coolant loop (large pipe diameters and relatively small length-to-diameter ratios).

\section{PIPING FLEXIBILITY STRESS COMPUTER MODEL}

A finite-element model of a primary coolant loop was constructed on the bas is of the configuration and parameters of the Combustion Engineering (1977) design. The computer program ANSYS (DeSalvo and Swanson 1975) was used for the calculations. The objective was to establish a more detailed picture of axial loads and bending moments, and to establish the conservatism present in the above simplified model.

Analyses were performed for a uniform temperature rise of the entire system and individual temperature rises for the hot and cold legs of the loop. For the calculations reported here, the pumps were assumed to be completely unrestrained. Other calculations which included the vertical and horizontal columns as described by Combustion Engineering (1977) showed that some restraint may be present. This restraint could increase the stress levels by about $50 \%$ over the levels reported here.

Figures $B .2$ and $B .3$ show stresses arising from temperature changes in the hot leg and one cold leg, respectively. As indicated, stresses from pure axial loads are relatively low, being abour one-tenth of the stress due to bending moments in the piping. The highest stresses are calculated at the cold legto-steam generator junction. Levels of these stresses are less than those predicted above by the simplified planar model. 


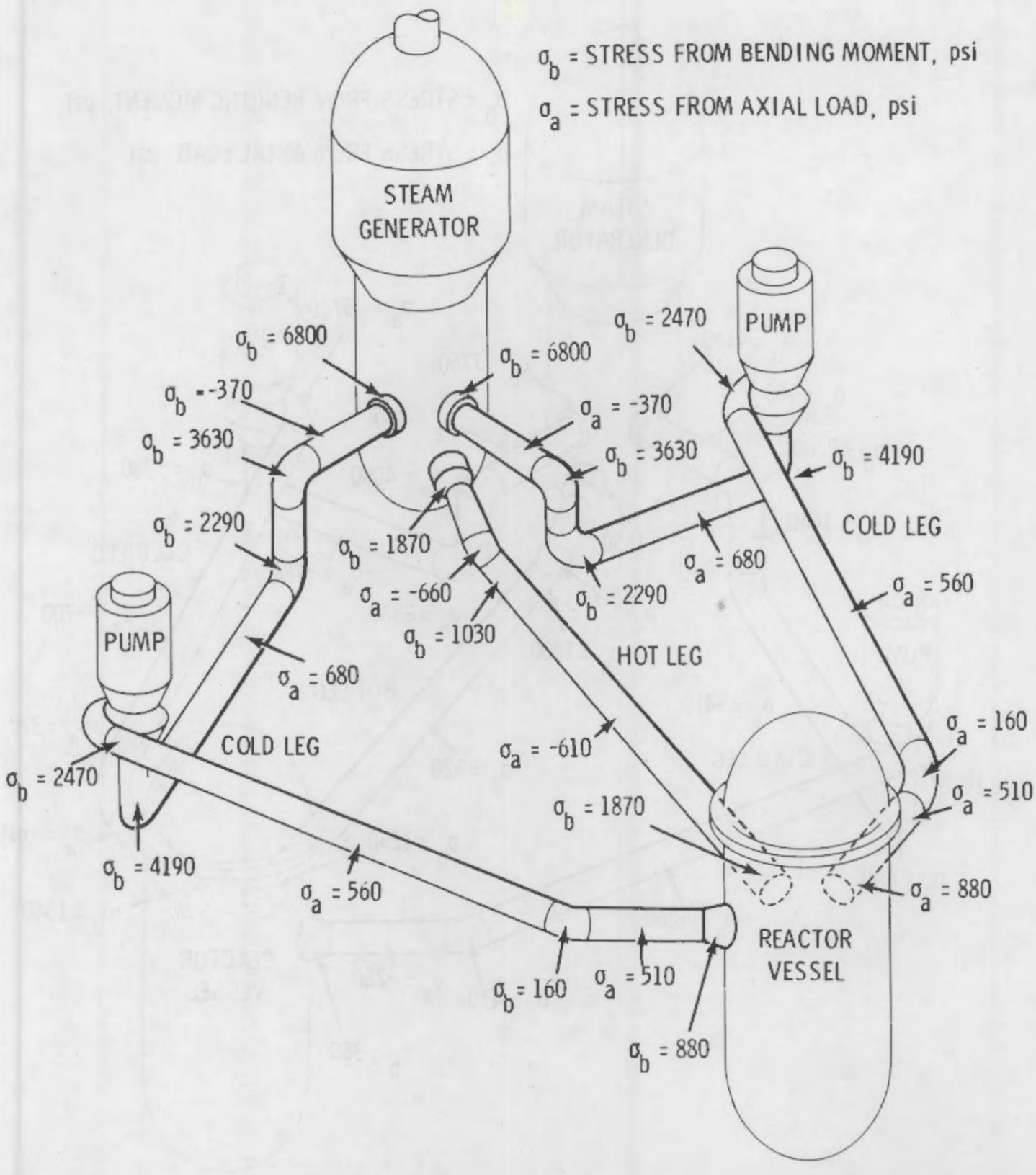

FIGURE B.2. Coolant Loop Stresses Calculated by Finite Element Analys is for $100^{\circ} \mathrm{F}$ Temperature Increase in Hot Leg 


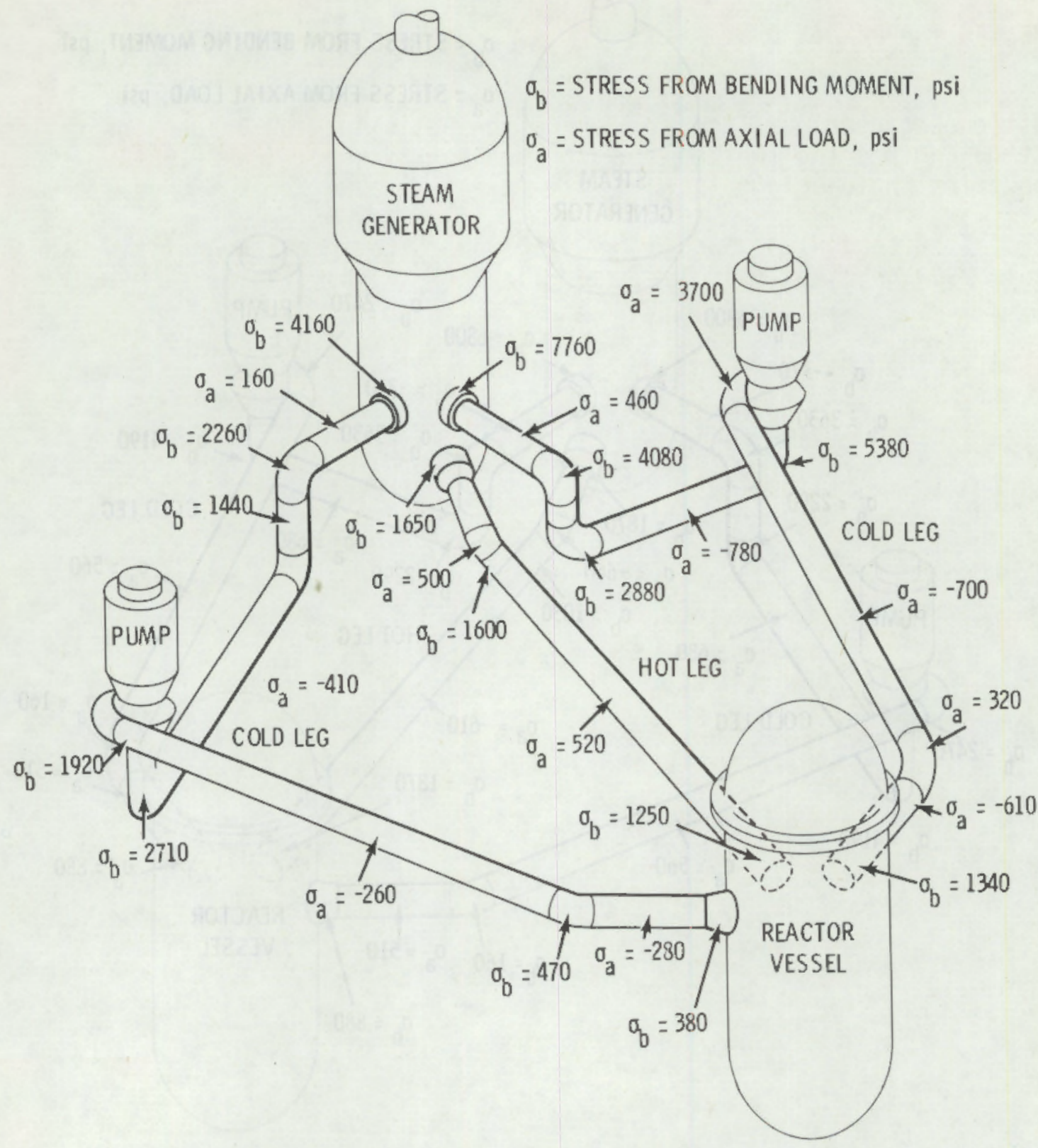

FIGURE B.3. Coolant Loop Stresses Calculated by Finite Element Analysis for $100^{\circ} \mathrm{F}$ Temperature Increase in One Cold Leg 
Combustion Engineering, Inc. 1977. Design Basis in Pipe Breaks for the Com bustion Engineering Two Loop Reactor Coolant System. CENPD-158-A, issued by Combustion Engineering, Inc.

DeSalvo, G. J. and J. A. Swanson. 1975. ANSYS Engineering Analysis User's Manual. Swanson Analysis Systems, Elizabeth, Pennsylvania.

Reed, R. P., H. I. McHenry, and M. B. Kasen. 1979. "A Fracture Mechanics Evaluation of Flaws in Pipeline Girth Welds." Welding Research Council Bullet in No. 245. 

APPENDIX C

LIST OF SAMPLES 
APPENDIX C

\section{LIST OF SAMPLES}

The objective of this appendix is to list and describe the test samples used in the Phase I program. A sample numbering system was developed to provide rapid identification of the samples. The numbering system is as follows:

$$
\frac{x X X X}{1}-\frac{x}{2}-\frac{L X X X}{3}
$$

1. A four-digit program identification number, 2289 for this program.

2. A single-digit indicating the year of manufacture, -9 for Phase I samples fabricated in 1979.

3. A letter followed by a three-digit identification code. The letter signifies the type of flaw in the sample.

The letter identification code is as follows:

A--samples defected by conventional fatigue process

B--samples defected by the thermal fatigue process

C--artificial flaws produced by machining, electrodischarge machining (EOM), or other mechanical methods.

The defecting processes used to fabricate type $A$ and $B$ samples are described in Section 6 of this report.

Tables C.1, C.2, and C. 3 describe the fatigue, thermal fatigue, and artificial flaws respectively. All of the samples were fabricated from a single heat of 1.0-in.-thick 304 stainless steel plate. Size refers to the finished sample dimensions.

\section{C.1}


Material Treatment describes pre- and post-defecting processes such as cold working and annealing. These treatments are described in Sections 6 and 7 of the main text of this report. The defecting process refers to the two methods described in Section 6.

Table C.3 lists samples fabricated by machining processes. Sketches of sample configurations are shown in Figure C.1. Dimensions of the notches were measured directly or from case replicas of the notch.

This list of samples will be updated as more samples are fabricated and will be published in future reports.
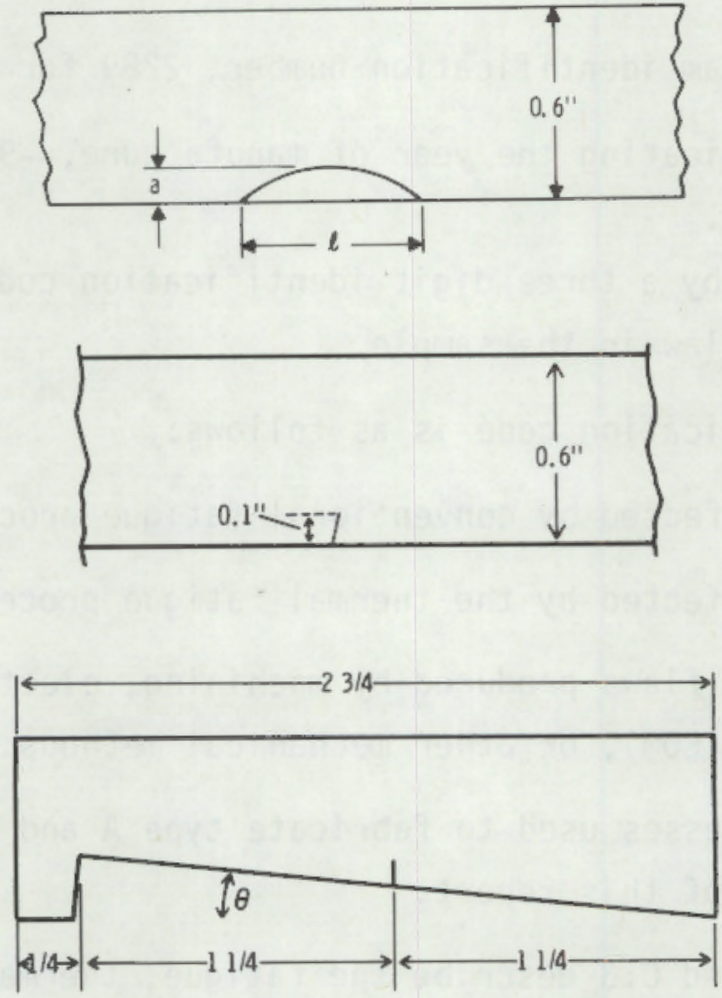

FIGURE C.1. EDM Notch Configurations 
TABLE C.1. Bending Fatigue Flaws

\begin{tabular}{|c|c|c|}
\hline & $\begin{array}{l}\text { Sample } \\
\text { Number } \\
\end{array}$ & Material \\
\hline & A139 & $3045 S$ \\
\hline & A140 & $3045 S$ \\
\hline & A141 & $304 S S$ \\
\hline & A142 & $304 S S$ \\
\hline & A143 & 30455 \\
\hline & A144 & $304 S S$ \\
\hline & A145 & $304 S S$ \\
\hline & A146 & $304 S S$ \\
\hline & A147 & $304 S S$ \\
\hline & A148 & $304 S S$ \\
\hline & A149 & 30455 \\
\hline & A 150 & $3045 s$ \\
\hline & A151 & $3045 S$ \\
\hline$?$ & A152 & $304 S S$ \\
\hline$\omega$ & A153 & 304 SS \\
\hline & A154 & $3045 S$ \\
\hline & A155 & 30455 \\
\hline & A156 & $3045 S$ \\
\hline & A157 & $304 S S$ \\
\hline & A158 & $304 S S$ \\
\hline & A159 & $304 S S$ \\
\hline & A160 & 304 SS \\
\hline & Al61 & $304 S S$ \\
\hline & A162 & $3045 S$ \\
\hline & A163 & $3045 S$ \\
\hline & A164 & $3045 S$ \\
\hline
\end{tabular}

\begin{tabular}{|c|c|c|c|c|c|c|c|c|c|c|}
\hline \multirow{2}{*}{\multicolumn{3}{|c|}{$\frac{\text { Size (in.) }}{\text { Length Width Thick }}$}} & \multirow{3}{*}{$\begin{array}{c}\text { Material } \\
\text { Treatment }(a) \\
\end{array}$} & \multirow{3}{*}{\multicolumn{2}{|c|}{$\begin{array}{l}\text { Defect } \\
\text { Process }\end{array}$}} & \multicolumn{4}{|c|}{ Flaw Dimensions (in.) } & \multirow[b]{3}{*}{ Conments } \\
\hline & & & & & & \multirow{2}{*}{$\begin{array}{l}\text { PT } \\
\text { Length } \\
\end{array}$} & \multirow{2}{*}{$\begin{array}{c}\text { ERG } \\
\text { Depth }\end{array}$} & \multirow{2}{*}{$\begin{array}{r}\text { Act } \\
\text { Length }\end{array}$} & & \\
\hline Length & Width & Thick & & & & & & & \multirow{2}{*}{$\frac{\text { Depth }}{\text { NA }}$} & \\
\hline 19 & 4.175 & 0.660 & CW & $B F$ & Sch. A & & 0.120 & NA & & \\
\hline 19 & 4.235 & 0.665 & $\mathrm{CW}$ & BF & Sch. A & 1.280 & 0.205 & 1.280 & 0.226 & Discontinuous Flaw \\
\hline 19 & 4.250 & 0.651 & $\mathrm{CW}$ & $B F$ & Sch. A & 1.305 & 0.245 & 1.305 & 0.232 & \\
\hline 19 & 4.315 & 0.642 & A & $B F$ & Sch. A & 0.672 & 0.120 & 0.645 & 0.121 & Continuous Flaw \\
\hline 19 & 4.138 & 0.630 & A & BF & Sch. A & 0.390 & 0.088 & 0.372 & 0.086 & Discontinous $\mathrm{Flaw}$ \\
\hline 19 & 4.155 & 0.606 & A & $F B$ & Sch. A & 0.978 & 0.180 & 0.968 & 0.177 & Continuous Flaw \\
\hline 19 & 4.20 & 0.625 & $\mathrm{CW}$ & $B F$ & Sch. A & 1.065 & 0.205 & NA & NA & Discont inuous $\mathrm{Flaw}$ \\
\hline 19 & 4.236 & 0.615 & CW & $B F$ & Sch. A & 0.284 & 0.080 & NA & NA & Continuous Flaw \\
\hline 19 & 4.175 & 0.615 & $\mathrm{CW}$ & BF & Sch. A & 0.715 & 0.110 & NA & NA & Continuous Flaw \\
\hline 19 & 4.215 & 0.627 & $\mathrm{CW}$ & $B F$ & Sch. A & 0.792 & 0.135 & 0.776 & 0.141 & Continuous Flaw \\
\hline 19 & 4.258 & 0.636 & $\mathrm{CW}$ & $B F$ & Sch. A & 0.346 & 0.070 & NA & NA & Discontinuous Flaw \\
\hline 19 & 4.18 & 0.655 & $\mathrm{CW}$ & $B F$ & Sch. A & 1.275 & 0.210 & NA & NA & Discontinuous Flaw \\
\hline 19 & 4.305 & 0.630 & CW & $B F$ & Sch. A & 0.314 & 0.080 & NA & NA & Continuous Flaw \\
\hline 19 & 4.235 & 0.635 & $\mathrm{CH}$ & BF & Sch. 8 & 0.352 & 0.080 & NA & NA & Discontinuous Flaw \\
\hline 19 & 4.14 & 0.625 & CW & $B F$ & Sch. B & 0.284 & 0.060 & 0.268 & 0.068 & Discontinuous Flaw \\
\hline 19 & 4.17 & 0.635 & $\mathrm{CW}$ & $B F$ & Sch. A & 1.221 & 0.210 & NA & NA & $\begin{array}{l}\text { Discontinuous near } \\
\text { one end of the flaw }\end{array}$ \\
\hline 19 & 4.20 & 0.622 & A & BF & Sch. B & 0.330 & 0.075 & 0.325 & 0.091 & Continuous Flaw \\
\hline 19 & 4.155 & 0.628 & A & $B F$ & Sch. B & 0.330 & 0.070 & 0.322 & 0.090 & Continuous Flaw \\
\hline 19 & 4.175 & 0.600 & $\mathrm{CW}$ & $B F$ & Sch. B & 0.311 & 0.055 & NA & NA & Discontinuous Flaw \\
\hline 19 & 4.11 & 0.620 & $\mathrm{CW}$ & $B F$ & Sch. B & 0.318 & 0.060 & NA & NA & Discontinuous Flaw \\
\hline 19 & 4.15 & 0.630 & $\mathrm{CW}$ & $B F$ & Sch. 8 & 1.355 & 0.230 & NA & NA & Continuous Flaw \\
\hline 19 & 4.20 & 0.676 & $\mathrm{CW}$ & $B F$ & Sch. B & 0.858 & 0.200 & 0.851 & 0.136 & Discontinuous Flaw \\
\hline 19 & 4.24 & 0.630 & $\mathrm{CH}$ & BF & Sch. B & 1.432 & 0.230 & 1.470 & 0.235 & Discontinuous Flaw \\
\hline 19 & 4.16 & 0.688 & $\mathrm{CW}$ & $B F$ & Sch. B & 0.908 & 0.180 & NA & NA & Discontinuous Flaw \\
\hline 19 & 4.15 & 0.617 & A & BF & Sch. B & 0.574 & 0.100 & 0.562 & 0.079 & Cont inuous Flaw \\
\hline 19 & 4.1 & 0.681 & CW & $B F$ & Sch. B & 0.643 & 0.120 & NA & NA & \\
\hline
\end{tabular}

(a) $\mathrm{CW}=$ Cold worked

(b) $\mathrm{SC}=$ Saw Cut

$E D M=$ Electric discharge machine

$T F=$ Thermal fatigue

$B F=$ Bending fatigue, Schedule $A$ or $B ; A=$ Rough $B=$ Smooth 
TABLE C.2. Thermal Faticue Flaws

\begin{tabular}{|c|c|c|c|c|c|c|c|c|c|c|c|}
\hline \multirow{2}{*}{$\begin{array}{l}\text { Sample } \\
\text { Number } \\
\end{array}$} & \multirow[b]{2}{*}{ Material } & \multicolumn{3}{|c|}{ Size (in.) } & \multirow{2}{*}{$\begin{array}{l}\text { Material }(a) \\
\text { Treatment }\end{array}$} & \multirow{2}{*}{$\begin{array}{l}\text { Defect } \\
\text { Process }(b) \\
\end{array}$} & \multicolumn{4}{|c|}{ Flaw Dimensions (in.) } & \multirow[b]{2}{*}{ Comments } \\
\hline & & Length & Width & Thick & & & & Depth & Length & Depth & \\
\hline B101 & $304 S 5$ & 11 nom. & 4.19 & 0.750 & $\mathrm{CW}$ & TF & & 0.135 & 0.827 & 0.262 & . \\
\hline B102 & 30455 & 11 nom. & 4.14 & 0.730 & $\mathrm{CW}$ & TF & 0.570 & 0.120 & & & Discontinuous Flaw \\
\hline B103 & $304 S S$ & 11 nom. & 4.14 & 0.752 & $\mathrm{CW}$ & TF & & 0.200 & 0.70 & 0.193 & \\
\hline B104 & $3045 S$ & 11 nom. & 4.18 & 0.731 & $\mathrm{CW}$ & TF & & 0.400 & 0.825 & 0.158 & \\
\hline B105 & 30455 & 11 nom. & 4.24 & 0.731 & $\mathrm{CW}$ & TF & 0.400 & 0.060 & & & \\
\hline B106 & $3045 S$ & 11 nom. & 4.17 & 0.728 & $\mathrm{CW}$ & TF & 0.460 & 0.090 & & & \\
\hline B113 & 304 SS & 11 nom. & 4.27 & 0.635 & A & TF & 0.570 & 0.100 & & & \\
\hline B114 & $304 S S$ & 11 nom. & 4.23 & 0.656 & A & TF & & 0.120 & 0.572 & 0.147 & \\
\hline B115 & 30455 & 11 nom. & 4.30 & 0.630 & A & TF & 0.500 & 0.055 & & & \\
\hline 8116 & $3045 S$ & 11 nom. & 4.27 & 0.650 & A & TF & 0.515 & 0.140 & & & \\
\hline B117 & $3045 S$ & 11 nom. & 4.31 & 0.644 & $\begin{array}{l}\text { A before and } \\
\text { after }\end{array}$ & TF & 0.760 & 0.240 & & & \\
\hline B118 & $3045 S$ & 11 nom. & 4.23 & 0.604 & $\begin{array}{l}\text { A before and } \\
\text { after }\end{array}$ & TF & 1.09 & 0.310 & & & \\
\hline (a) $\mathrm{CW}$ & $\begin{array}{l}\text { Cold work } \\
\text { Annealed }\end{array}$ & & & $\begin{aligned} S C & = \\
E D M & = \\
T F & = \\
B F & =\end{aligned}$ & $\begin{array}{l}\text { Saw Cut } \\
\text { Electric disch } \\
\text { Thermal fatigu } \\
\text { Bending fatigue }\end{array}$ & $\begin{array}{l}\text { ge machine } \\
\text { Schedule A }\end{array}$ & & ough & $=$ Smoot & & \\
\hline
\end{tabular}


TABLE C.3. Artificial Flaws

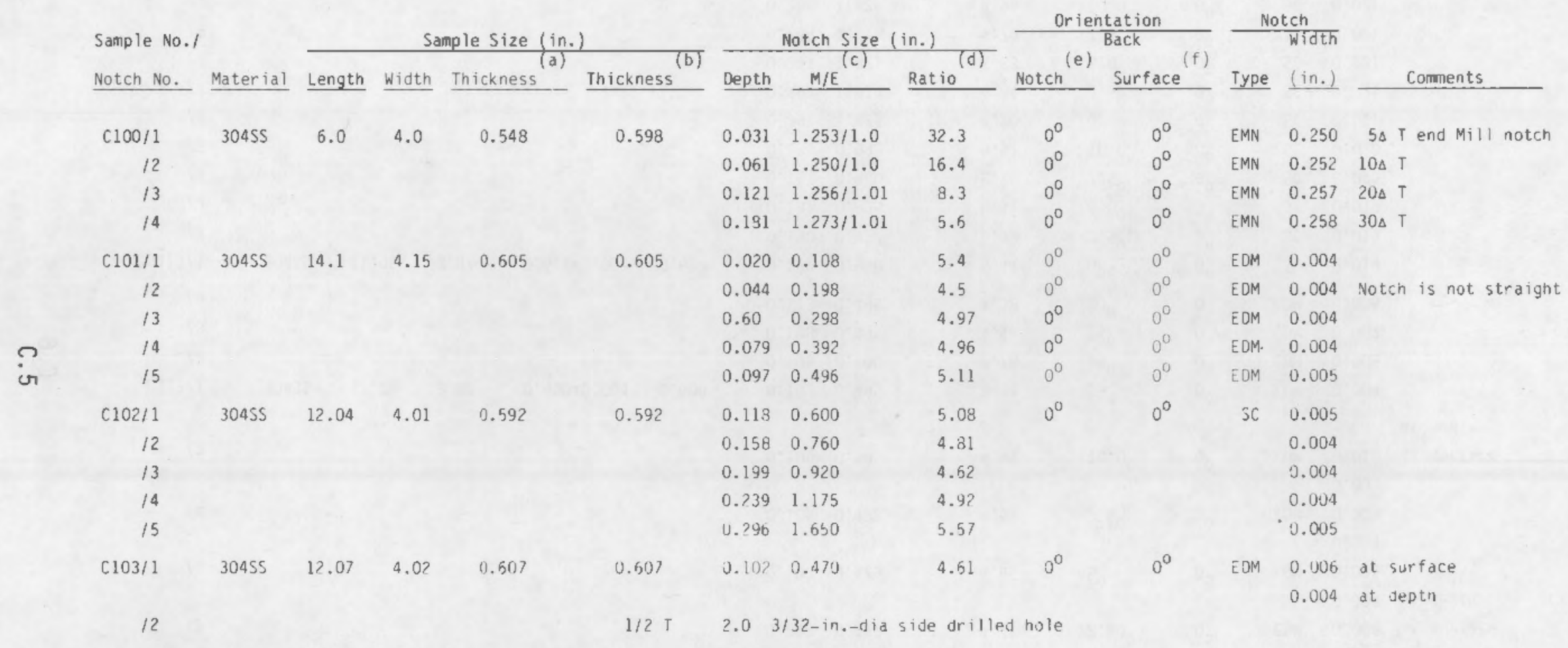

\footnotetext{
(a) Maximum thickness.

(b) Thickness at Notch.

(c) Maximum/Effective.

(d) Length/Depth

(e) Degrees from perpendicular to front surface.

(f) Degrees from parallel to front surface.
} 
TABLE C.3. (contd)

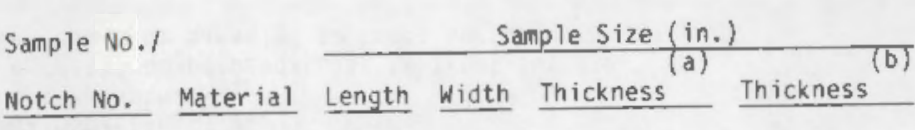
(c) (d)

$\frac{\text { Orientation }}{\text { Back }}$ (e)
Notch
Surface (f)

Comments

\begin{tabular}{|c|c|c|c|c|c|c|c|c|c|c|c|c|}
\hline$C 111 / 1$ & $304 \$ S$ & 12.09 & 4.025 & 0.602 & 0.602 & 0.101 & 0.473 & 4.68 & $0^{\circ}$ & $0^{\circ}$ & EOM & $\begin{array}{l}0.008 \\
0.004\end{array}$ \\
\hline 12 & & & & & & 0.098 & 0.462 & 4.71 & $2.50^{\circ}$ & $0^{0}$ & EOM & 0.008 \\
\hline 13 & & & & & & 0.103 & 0.479 & 4.65 & $5^{0}$ & $0^{\circ}$ & EOM & $\begin{array}{l}0.004 \\
0.005\end{array}$ \\
\hline 14 & & & & & & 0.105 & 0.482 & 4.59 & $7.5^{\circ}$ & $0^{\circ}$ & EOM & $\begin{array}{l}0.004 \\
0.009\end{array}$ \\
\hline 15 & & & & & & 0.109 & 0.50 & 4.59 & $10.0^{\circ}$ & $0^{\circ}$ & EOM & $\begin{array}{l}0.003 \\
0.010\end{array}$ \\
\hline$C 112 / 1$ & 30455 & 12.0 & 3.98 & $0.600 \pm 0.004$ & 0.600 & 0.103 & 0.495 & 4.81 & $15^{\circ}$ & $0^{0}$ & EOM & 0.004 \\
\hline 12 & & & & & & 0.105 & 0.500 & 4.76 & $20^{\circ}$ & $0^{0}$ & EOM & 0.005 \\
\hline 13 & & & & & & 0.108 & 0.495 & 4.58 & $25^{\circ}$ & $0^{\circ}$ & EDM & 0.007 \\
\hline 14 & & & & & & 0.113 & 0.495 & 4.38 & $30^{\circ}$ & $0^{0}$ & EOM & 0.006 \\
\hline $\mathrm{C} 113 / 1$ & $304 \$ S$ & 11.96 & 3.97 & $0.612 \pm 0.002$ & 0.612 & 0.182 & 0.990 & 5.44 & $0^{\circ}$ & $0^{0}$ & SC & 0.019 \\
\hline 12 & & & & & & 0.180 & 0.977 & 5.43 & $2.5^{\circ}$ & $0^{0}$ & SC & 0.019 \\
\hline 13 & & & & & & 0.182 & 0.985 & 5.41 & $5.0^{\circ}$ & $0^{\circ}$ & SC & 0.019 \\
\hline 14 & & & & & & 0.167 & 0.960 & 5.75 & $7.5^{\circ}$ & $0^{0}$ & SC & 0.020 \\
\hline 15 & & & & & & 0.180 & 0.992 & 5.51 & $10.0^{\circ}$ & $0^{0}$ & SC & 0.020 \\
\hline 16 & & & & & & 0.297 & 1.425 & 4.90 & $0^{\circ}$ & $0^{\circ}$ & SC & 0.021 \\
\hline 17 & & & & & & 0.292 & 1.414 & 4.84 & $2.5^{\circ}$ & $0^{0}$ & SC & 0.021 \\
\hline 13 & & & & & & 0.294 & 1.421 & 4.83 & $5.0^{\circ}$ & $0^{0}$ & SC & 0.021 \\
\hline 19 & & & & & & 0.296 & 1.419 & 4.79 & $7.5^{\circ}$ & $0^{0}$ & SC & 0.021 \\
\hline 110 & & & & & & 0.288 & 1.426 & 4.95 & $10.0^{\circ}$ & $0^{\circ}$ & SC & 0.021 \\
\hline
\end{tabular}

(a) Maximum thickness.

b) Thickness at Notch.

c) Maximum/Effective.

(d) Length/Depth.

(e) Degrees from perpendicular to front surface.

(f) Degrees from parallel to front surface. 


\section{TABLE C.3. (contd)}

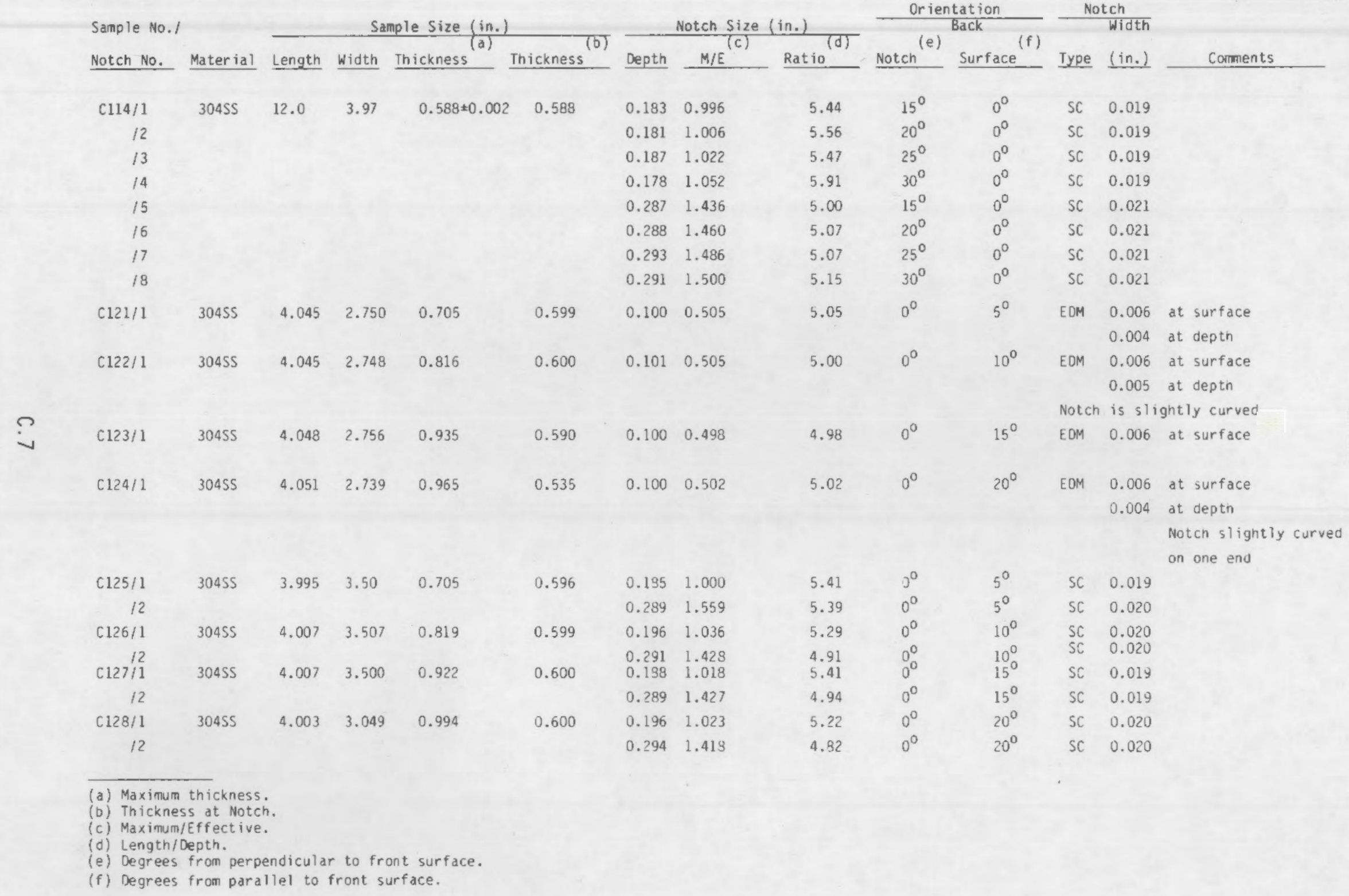





\section{APPENDIX D}

ANALYSIS OF THE THERMAL FATIGUE PROCESS

FOR CRACKED SPECIMEN PROOUCTION 
APPENDIX D

ANALYSIS OF THE THERMAL FATIGUE PROCESS

FOR CRACKED SPECIMEN PROOUCTION

One of the requirements of the present NRC-sponsored program on NDE raliability and fracture mechanics is to prepare cracked specimens. These specimens will be used to evaluate crack detection capabilities of ultrasonic inspection procedures.

It has been proposed to adapt a thermal fatigue method previously used by General Electric (G.E.) to initiate and grow cracks. This metnod is shown in schematic form in Figure D.1. A plate is heated on the back side to an elevated temperature (e.g., $\left.1100^{\circ} \mathrm{F}\right)$, and a local area (1.16 in. dia) is cooled with water jet on the opposite side of the plate. A $0.75-i n$. long by $0.02-$ to 0.04-in. deep notch serves to initiate a crack. The heated plate is quenched for $15 \mathrm{sec}$ followed by a water-off period of $15 \mathrm{sec}$. General Electric has been able to grow cracks to lengths on the order of $1.0 \mathrm{in}$. with a few thousand cycles of quenching. The plates have been low carbon or low alloy steel in thicknesses of 2.0 and 6.0 in.

The Pacific Northwest Laboratory proposes to apply the G.E. method to somewhat different types of specimens. Wall thicknesses are as low as 0.500 in., and in some cases a pipe geometry rather than a flat plate will be utilized. In addition, some specimens will be of 304 stainless steel. Metallurgical considerations may dictate the use of preheat temperatures less than the $1100^{\circ} \mathrm{F}$ level used by G.E.

The study reported here was to determine if the G.E. method can crack the specimens of PNL's program with a reasonable number of thermal fatigue cycles. Approximate stress analyses were performed to predict trends in crack growth rates. The objective was to determine if cracks will grow faster or slower for 


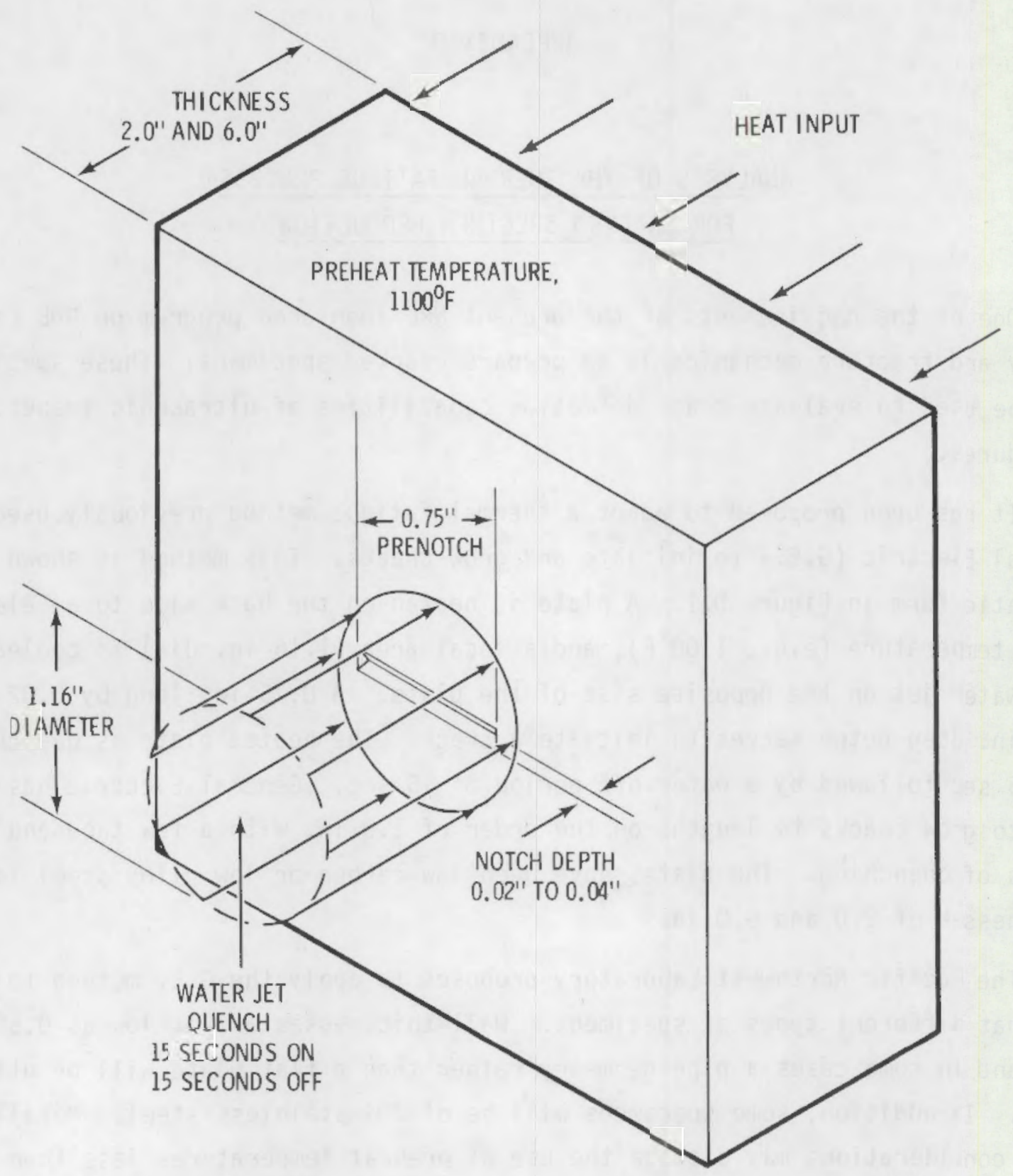

FIGURE 0.1. Thermal Fatigue Cracking Specimen

PNL's conditions as opposed to those within G.E.'s experience. The calculations are intended to guide and complement empirical cracking studies to be performed at Battelle in an experimental facility which will be based on the G.E. design. 
Details of the analyses method are first described below. Results and conclusions are presented in the final section under the title of parametric studies.

\section{HEAT TRANSFER}

The idealized heat transfer model shown in Figure 0.2 permitted trends relative to wall thickness and material thermal properties to be considered. Closed form solutions for transient heat conduction could be utilized since the heat transfer was purely one dimensional (i.e., through the thickness of the plate).

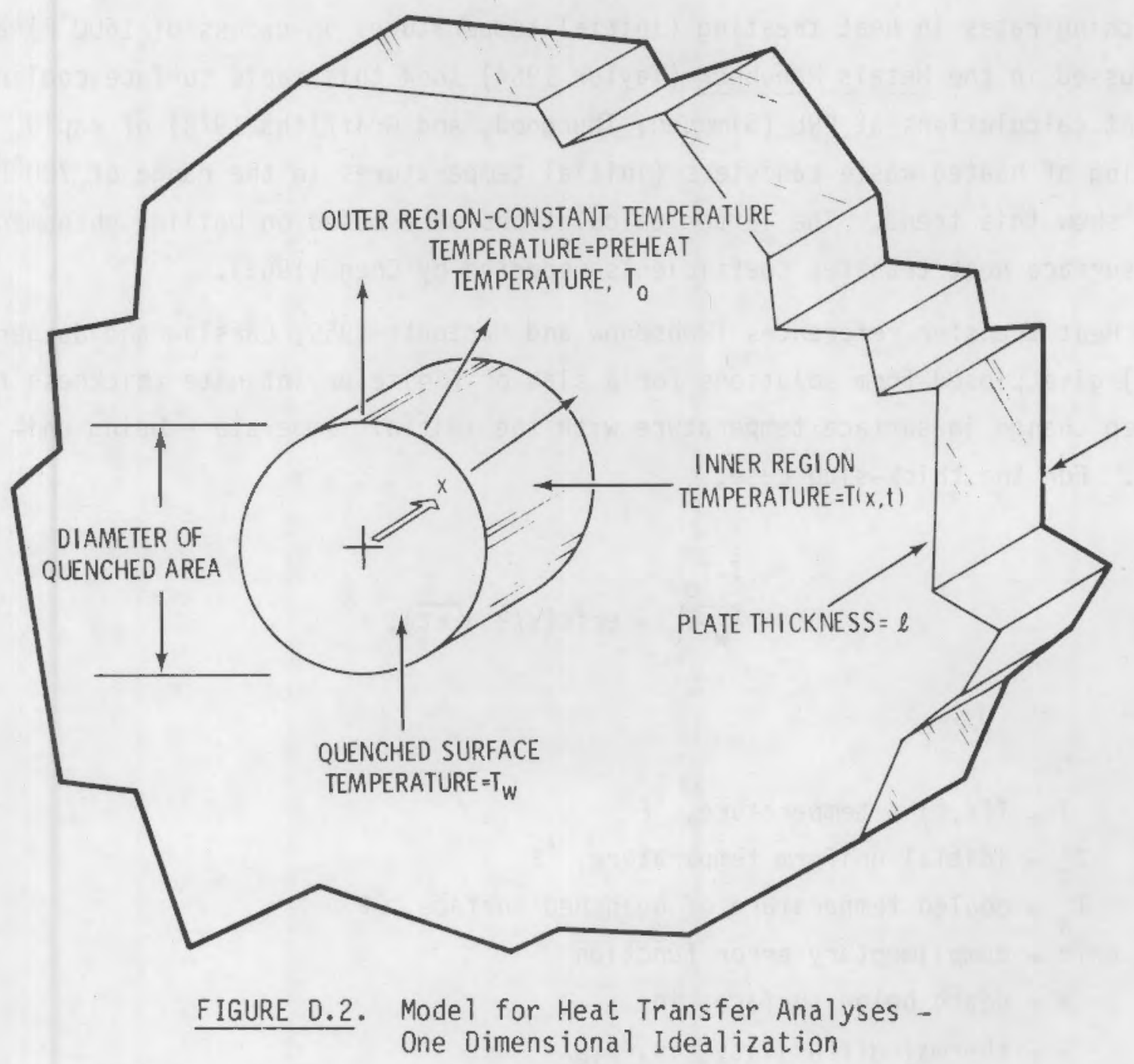


The analysis considered only the first thermal shock, when the initially hot plate was cooled locally by the water jet. Subsequent reheating once the jet was turned off and the eventual periodic temperature variation was not considered. Also inherent to the one-dimensional analysis was the neglect of heat flow parallel to the plane of the plate. It is believed, nevertheless, that the selected representation of the thermal shock conditions provided a sound bas is for establishing comparative stress levels.

Available data indicate that the area of hot metal surface exposed to the water jet will cool to temperature at or below $200^{\circ} \mathrm{F}$ in less than one second. Accordingly the surface was taken to be cooled instantaneously. Data on quenching rates in heat treating (initial temperatures in excess of $1600^{\circ} \mathrm{F}$ ) as discussed in the Metals Handbook (Taylor 1964) show this rapid surface cooling. Recent calculations at PNL (Simonen, Thurgood, and Griffiths 1978) of rapid cooling of heated waste canisters (initial temperatures in the range of $700^{\circ} \mathrm{F}$ ) also show this trend. The latter calculations were based on boiling phenomena and surface heat transfer coefficients reported by Chen (1963).

Heat transfer references (Rohsenow and Hartnett 1959; Carslaw and Jaeger 1959) give closed-form solutions for a slab of finite or infinite thickness for a step change in surface temperature with the initial temperature being uni-form. For the thick-slab case,

$$
\frac{T-T_{0}}{\bar{T}^{-T_{0}}}=\operatorname{erfc}(x / 2 \sqrt{k t}),
$$

where

$$
\begin{aligned}
T & =T(x, t)=\text { temperature, }{ }^{\circ} \mathrm{F} \\
T_{0} & =\text { initial uniform temperature, }{ }^{\circ} \mathrm{F} \\
T_{W} & =\text { cooled temperature of quenched surface, }{ }^{\circ} \mathrm{F} \\
\text { erfc } & =\text { complimentary error function } \\
x & =\text { depth below surface, in. } \\
\kappa & =\text { thermal diffusivity, in. }{ }^{2} / \mathrm{sec} \\
t & =\text { time, seconds. }
\end{aligned}
$$


This solution gives accurate results for the finite thickness slab for times soon after the quench, but does not account for back-side effects which develop after longer times.

A series solution which converges slowly for small values of time, but includes back surface effects is given by Carslaw and Jaeger (1959) as follows:

$$
\frac{T-T_{0}}{T_{w}^{-T_{0}}}=(4 / \pi) \quad \sum_{n=0}^{\infty} \frac{1}{(2 n+1)} e^{-k(2 n+1)^{2} \pi^{2} t / e^{2} \sin (2 n+1) x / \ell}
$$

where $\ell$ is the wall thickness. The back surface is assumed to be insulated, which neglects the transfer of heat into the plate over the small time period of the quench.

The above solutions for cooling for the initial quench will tend to overestimate the severity of quenching under conditions of repeated quenching. After a number of quenches, locations in the cooled zone will attain some mean temperature below the remote temperature $T_{0}$. Thus, the effective value of initial temperature will be somewhere between $T_{0}$ and $T_{W}$.

\section{STRESS ANALYSIS}

The stress analysis was based on the elasticity theory solution for a circular disc in a hole in a plate as indicated in Figure D.3. It is implied that each mathematical layer of the plate acts independently of the others. That is, shear stresses between layers do not restrain adjacent layers. This approach will tend to underestimate stresses perhaps by a factor as high as two. On the other hand, the heat transfer solution by not considering effects of repeated quenching may overestimate the thermal loads by a comparable factor of two.

As shown in Figure 0.3 , the quenched circular disc region experiences a thermal contraction strain of

$$
{ }^{\varepsilon} \text { thermal }=\alpha\left(T-T_{0}\right)
$$




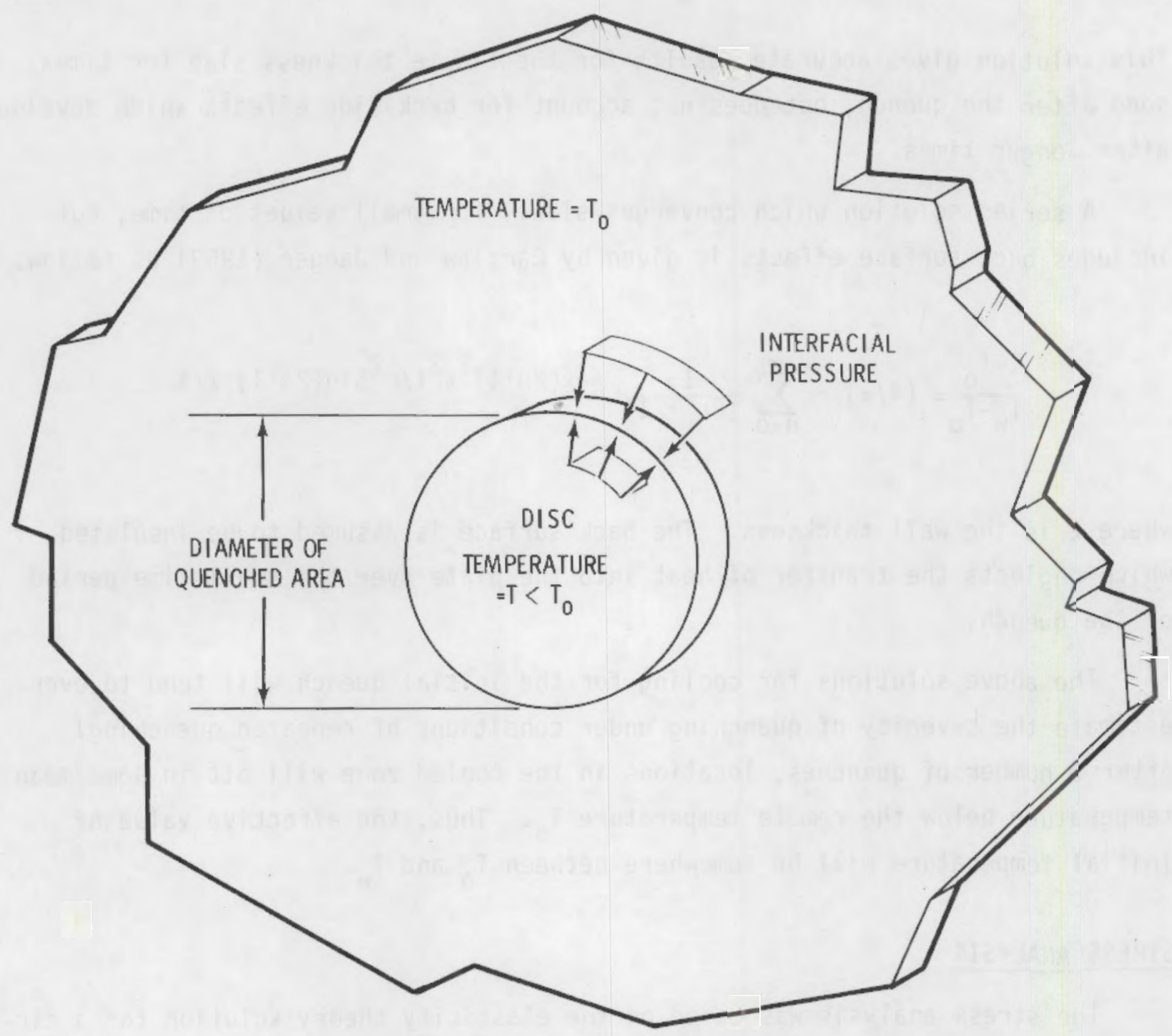

FIGURE D.3. Model for Stress Analys is - Thermal Contraction of Disc in Hole

$\alpha=$ thermal expansion coefficient, in. $/$ in. $/^{\circ} \mathrm{F}$

relative to the surrounding region that remains at $T_{0}$. To accommodate the differential thermal strain, mechanical strains in the disc and surrounding plate must satisfy the condition

$$
E_{\text {plate }}{ }^{-\varepsilon_{\text {disc }}}=-\varepsilon_{\text {thermal }}
$$


where

$$
\begin{aligned}
\varepsilon_{\text {disc }} & =-(p / E)(1-v) \\
\varepsilon_{\text {plate }} & =+(p / E)(1+v) \\
p & =\text { radial pressure at interface } \\
E & =\text { elastic modulus } \\
v & =\text { Poisson's Ratio }
\end{aligned}
$$

The stress within the quenched region is given by

$$
\sigma=\sigma(x, t)=-p=1 / 2 E \alpha(T-J)
$$

This stress was used as an input to estimate stress intensity factors for cracks of various depths.

\section{CRACK TIP STRESS INTENSITY}

Stress intensity factors corresponding to the thermal stress distributions were computed using the idealization shown in Figure D.4. Stresses from the uncracked thermal stress solution were applied as loadings on the faces of an edge crack. The edge crack solution reasonably represents the cracked specimen geometry for short cracks. However, for deeper cracks, the effects of crack aspect ratio is lost (e.g., an elliptical surface crack). This edge crack simplification is believed to be consistent with the types of approximations made in other aspects of the analysis.

In reference to Figure 0.4 , the crack face stresses were approximated as a set of point loads. Using a solution reported in Tada (1973), the stress intensity factor $K_{I}$ was computed as

$$
K_{I}=\frac{2}{\pi a} \sum_{i=1}^{N} P_{i} F\left(b_{i} / a\right) \sqrt{1-\left(b_{i} / a\right)^{2}},
$$

where $a, b$, and $P$ are defined in Figure 0.4 . And $F\left(b_{i} / a\right)$ is a function defined in Tada (1973) which varies from 1.0 for $b / a=1.0$ to 1.3 for $b / a=0$. 


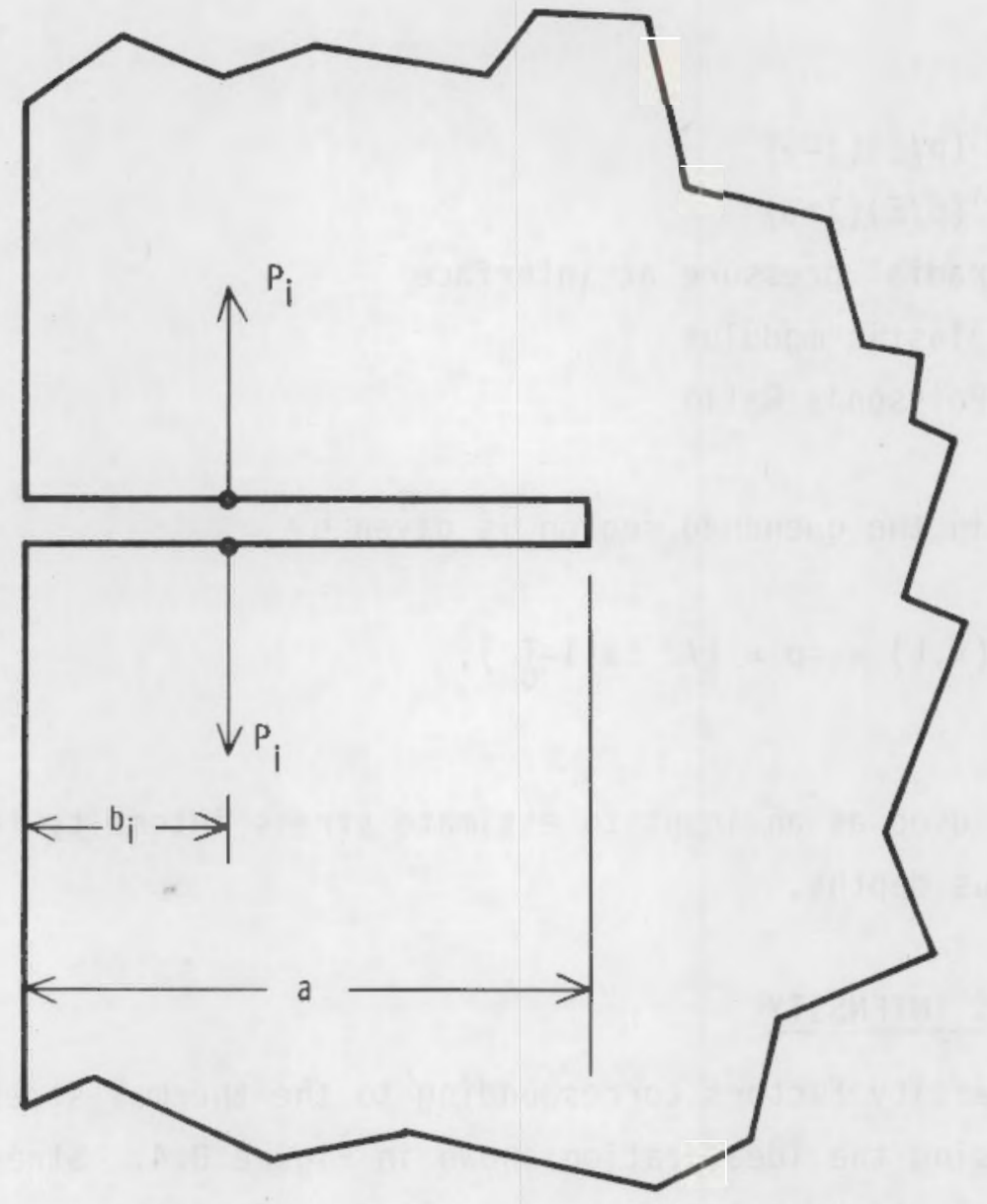

\section{FIGURE 0.4. Model for Calculating Stress Intensity Factors}

The above procedure for stress intensity evaluation along with the necessary heat transfer and stress equations were programmed for computer calculations, and parametric studies were performed.

Input parameters to the program included:

- plate thickness

- specimen preheat temperature

- temperature of cooled surface

- material properties - elastic modules, expansion coefficient and thermal diffusivity 
- cooling times of interest

- crack lengths of interest.

As output the program provides

- temperatures and stresses as function of position and time

- stress intensity factors as a function of crack length and time.

\section{PARAMETRIC STUDIES}

The following parameters were selected for study:

- plate thickness - 6.0,2.0, and 0.594 in.

- crack depth $-0.05,0.10,0.20,0.50,1.0,2.0$, and 4.0 in.

- materials - carbon steel and austenitic stainless steel with properties as given in the ASME Code

- preheat temperature $-1100^{\circ}$ and $650^{\circ} \mathrm{F}$

- cooled surface temperature $-200^{\circ} \mathrm{F}$

- cooling time - 1, 2, 5, 10, 15, 30, and $60 \mathrm{sec}$.

Variations in material properties with temperature were taken into account by selection of properties midway between $T_{0}\left(650^{\circ}\right.$ and $\left.1100^{\circ} \mathrm{F}\right)$ and $T_{W}\left(200^{\circ} \mathrm{F}\right)$. These values are given in Table 0.1 .

TABLE D.1. Thermal and Mechanical Properties from ASME Code

\begin{tabular}{|c|c|c|c|c|c|}
\hline & & & $\begin{array}{l}\text { Thermal } \\
\text { Expansion } \\
\text { Coefficient, } \\
\text { in. } / \text { in. }{ }^{\circ} \mathrm{F} \\
\end{array}$ & $\begin{array}{l}\text { Elastic } \\
\text { Modulus } \\
\text { lb/in. } \\
\end{array}$ & $\begin{array}{c}\text { Thermal } \\
\text { Diffusivity, } \\
\text { in. /sec }\end{array}$ \\
\hline Carbon & steel & $\begin{array}{l}425^{\circ} \mathrm{F} \\
650^{\circ} \mathrm{F}\end{array}$ & $\begin{array}{l}7.61 \times 10^{-6} \\
8.54 \times 10^{-6}\end{array}$ & $\begin{array}{l}26.8 \times 10^{6} \\
25.2 \times 10^{6}\end{array}$ & $\begin{array}{l}0.0263 \\
0.0167\end{array}$ \\
\hline \multirow[t]{2}{*}{ Austen i1 } & itic stainless & $425^{\circ} \mathrm{F}$ & $9.99 \times 10^{-6}$ & $26.5 \times 0^{6}$ & 0.0065 \\
\hline & & $650^{\circ} \mathrm{F}$ & $10.45 \times 10^{-6}$ & $25.6 \times 10^{6}$ & 0.0069 \\
\hline
\end{tabular}


Stress intensities were calculated based on linear elastic fracture mechanics, although nominal stresses exceeded yield for the imposed thermal shock conditions. Also, the various approximations in the analyses suggest that the calculated stress intensity factors may be accurate only within a factor of two. The results do, however, serve the objectives of the study by showing trends as a function of specimen and test parameters. More exact calculations would be feasible using finite element methods, but such methods were inconsistent with cost and schedule constraints of the present program.

\section{Effect of Plate Thickness}

Figure 0.5 shows stress intensities factors for plate thicknesses of 6.0 , 2.0, and 0.594 in. Within the approximations of the present analysis, no difference between the 2.0- and $5.0-i n$. thick plate were predicted. The thinner 0.594-in. plate showed slightiy higher stress intensity factors, particularly for deeper cracks and longer cooling times.

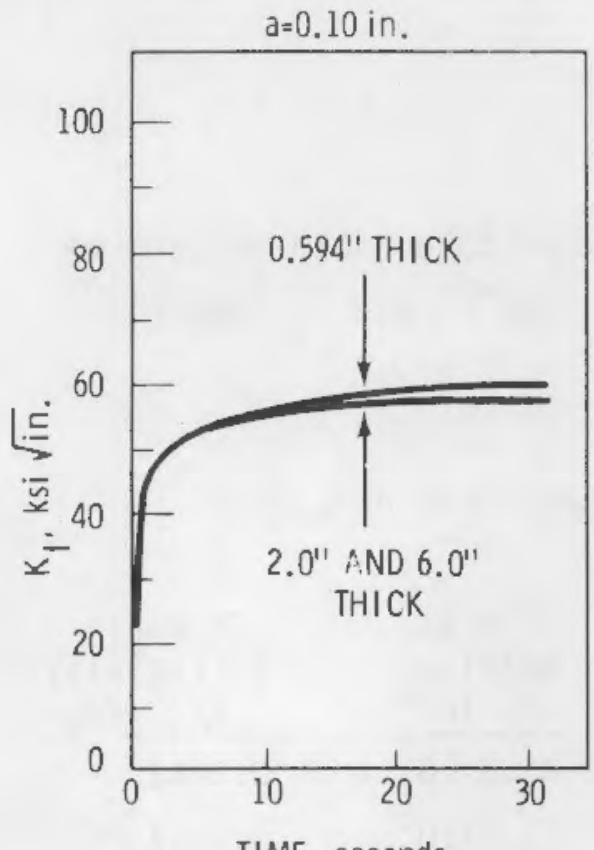

TIME, seconds

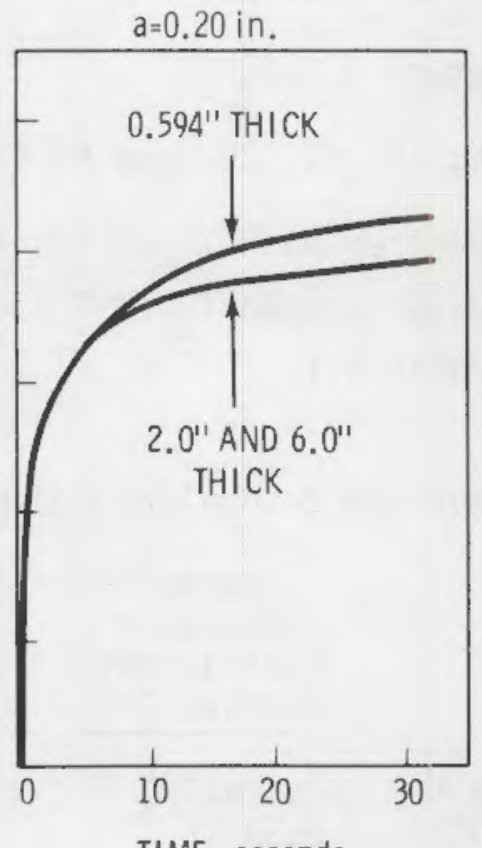

TIME, seconds

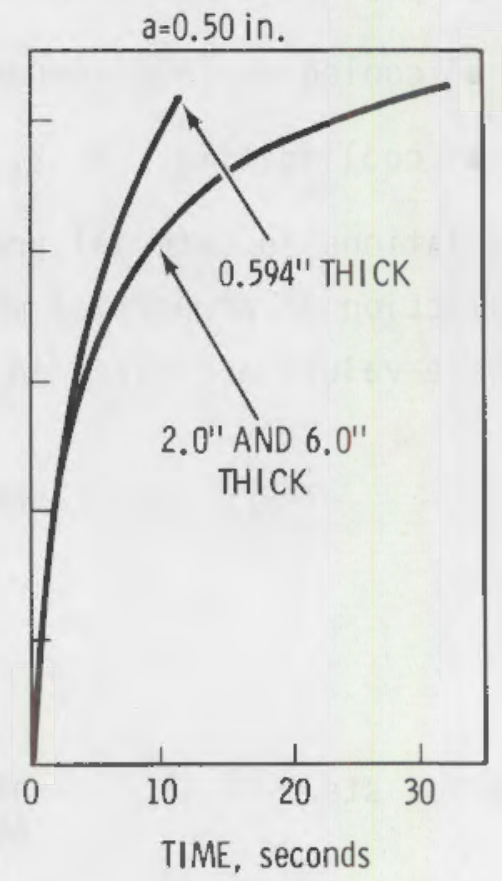

TIME, seconds

FIGURE 0.5. Effect of Thickness on Performance of Thermal Fatigue Cracking Specimen 
The 0.594 in. thickness corresponds to a pipe wall thickness of one proposed specimen. For the thermal fatigue conditions of interest it is believed that differences due to curvature between a pipe wall and a flat plate are of secondary importance.

Experience at G.E. has shown greater crack growth rates in 2.0-in. plates compared to 6.0-in. thick plates. These differences may be in part due to differences in the materials for the two plate thickness. Nevertheless, there could be a thickness effect not fully accounted for due to approximations in the present analyses. Particularly, the heat transfer model was only one dimensional and considered only the initial thermal shock. It is thought that the thicker plate acts as a better heat sink and more effectively reheats the quenched zone between water quenches. Thus, under repeated quenching the thicker plates probably have a higher effective temperature at the beginning of each quench.

Effect of Cooling Time

Figure D.5 shows that crack tip stress intensity factors increase as the quench duration increases. For the slower growing cracks of smaller depth, the stress intensity levels essentially saturate in the $15-\mathrm{sec}$ quench of the G.E. test procedures. The calculated trends suggest that a shorter quench, say $5 \mathrm{sec}$, may be adequate. In fact, short quenches at greater frequency may actually decrease the hours of test time to grow a crack of a given size. Effect of Preheat Temperature $\mathrm{T}_{0}$

Figure 0.6 shows that crack tip stress intensity factors are strongly dependent on preheat temperature. The dependence is essentially directly in proportion to the difference between the preheat and cooled temperature of the quenched surface $\left(200^{\circ} \mathrm{F}\right.$ in these calculations). Crack growth rates, being logarithmic functions of stress intensity factor, will decrease dramatically with reductions in preheat temperature $T_{0}$. Therefore, if cracked specimens are to be produced within a minimum of test time, the preheat temperature must be maintained at maximum practical levels. 
$\mathrm{a}=0.1 \mathrm{in}$.

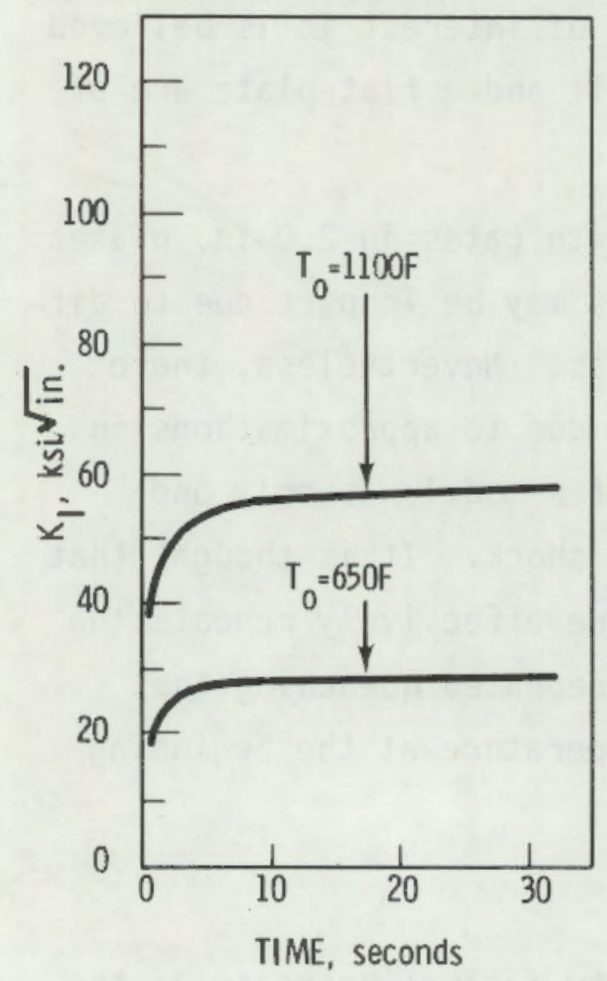

$\mathrm{a}=0.2 \mathrm{in}$.

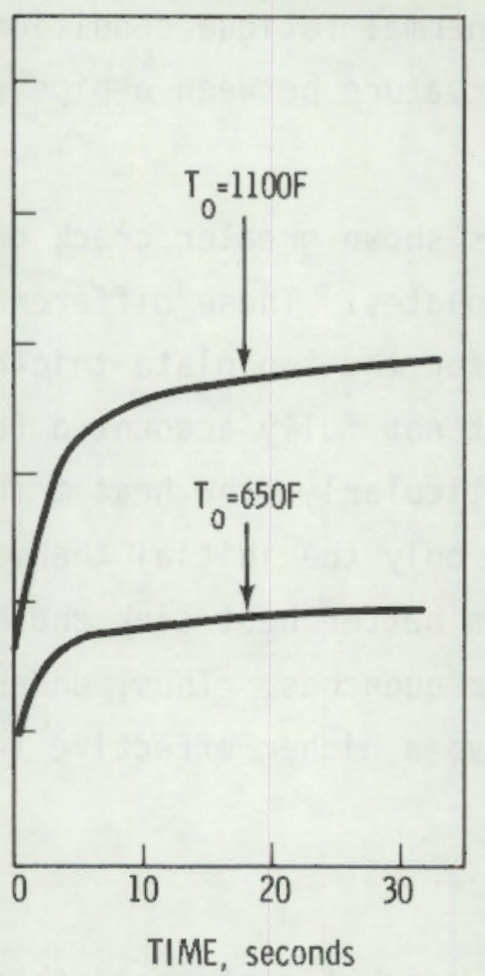

$\mathrm{a}=0.5 \mathrm{in}$.

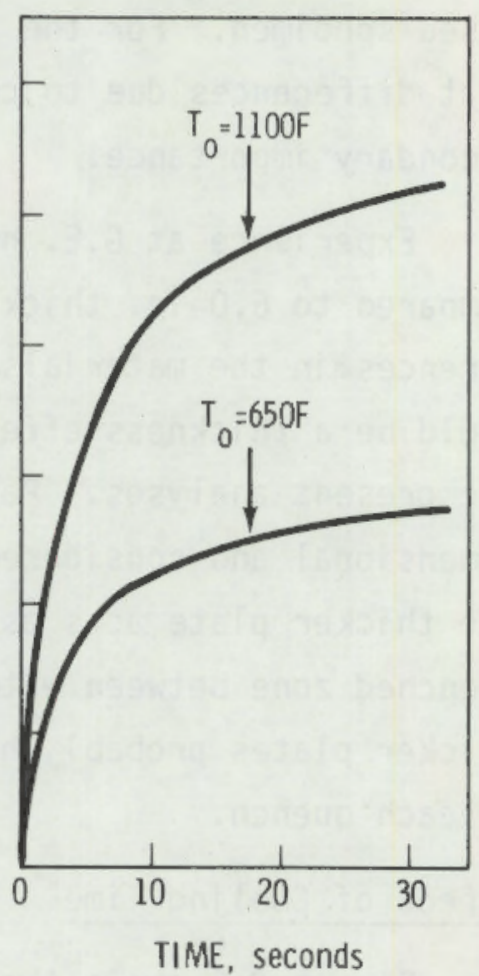

FIGURE 0.6. Effect of Preheat Temperature to Performance of Thermal Fatigue Cracking Specimen

\section{Effect of Material}

Figure 0.7 compares stress intensity factors for low-carbon (or low-alloy) steel with those for an austenitic stainless steel such as 304 . For a given plate thickness and quench, the stress intensity for the stainless alloys are somewhat higher than those for the low carbon type steels. This is due primarily to the higher thermal expansion coefficient of the stainless steel. However, the stainless steels have lower thermal conductivities, and Figure 0.7 shows a slower increase of stress intensity factor with time for the stainless steel. Fatigue crack growth rate data should first be located before drawing any conclusions on the ability of the thermal shock procedure to produce cracked specimens of stainless alloys. If low carbon steels and stainless steels have identical crack growth rate properties, then this study indicates that stainless specimens will be easier to crack. 

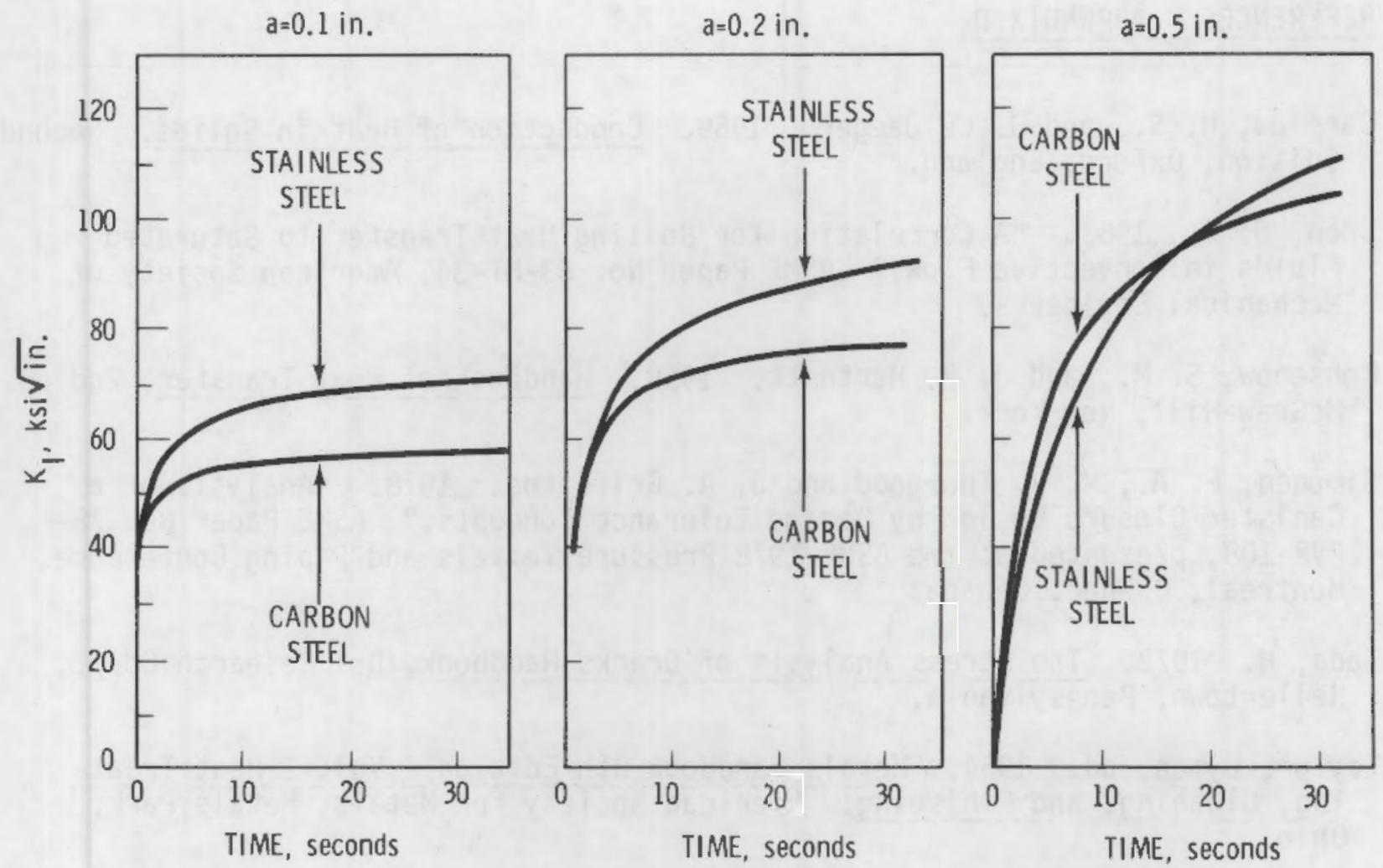

FIGURE 0.7. Effect of Specimen Material on Thermal Fatigue Cracking - Low Carbon Versus Austenitic Stainless Steel 
Carslaw, H. S., and J. C. Jaeger. 1959. Conduction of Heat in Solids. Second Edition, Oxf ord, England.

Chen, J. C. 1963. "A Correlation for Boiling Heat Transfer to Saturated Fluids in Convective Flow." ASME Paper No. 63-HT-34, American Society of Mechanical Engineers.

Rohsenow, S. M., and J. P. Hartnett. 1959. Handbook of Heat Transfer, 2nd ed. McGraw-Hill, New York.

Simonen, F. A., M. V. Thurgood and J. A. Griffiths. 1978. "Analysis of a Canister Closure Design by Damage Tolerance Concepts." ASME Paper No. 78PVP-108, presented at the ASME 1978 Pressure Vessels and Piping Conference, Montreal, Quebec, Canada.

Tada, H. 1973. The Stress Analysis of Cracks Handbook, Del Research Corp., Hellertown, Pennsylvania.

Taylor, Lyman, ed. 1964. Metals Handbook 8th Edition - Vol. 2 Heat Treating, Cleaning, and Finishing. Ainerican Society for MetaTs, Metals Park, onio. 
No. of

Copies

OFFSITE

A. A. Churm

DOE Patent Division

$9800 \mathrm{~S}$. Cass Avenue

Argonne, IL 60439

390

U.S. Nuclear Regulatory Commission

Division of Technical Information and Document Control

7920 Norfolk Avenue

Bethesda, MD 20014

2 DOE Technical Information Center

R. F. Abbey, Jr.

Reactor Safety Research Division

Nuclear Regulatory Commission

Washington, DC 20555

S. Fabric

Reactor Safety Research Division Nuclear Regulatory Commission Washington, DC 20555

D. A. Hoatson

Reactor Safety Research Division Nuclear Regulatory Commission Washington, DC 20555

W. V. Johnston

Reactor Safety Research Division Nuclear Regulatory Commission

Washington, DC 20555

10 J. Muscara

Reactor Safety Research Division Nuclear Regulatory Cormission Wash ington, DC 20555

R. D. Schamberger

Reactor Safety Research Division Nuclear Regulatory Commission Washington, DC 20555
No. of

Copies

H. H. Scott

Reactor Safety Research Division Nuclear Regulatory Cormission Washington, DC 20555

R. Van Houton

Reactor Safety Research Division Nuclear Regulatory Commission Washington, OC 20555

B. D. Liaw

Materials Engineering Branch

Division of Engineering

Nuclear Regulatory Division

Mail Stop P-1000

Washington, DC 20555

Martin R. Hum

Materials Engineering Branch

Division of Engineering

Nuclear Regulatory Division

Mail Stop P-1000

Washington, OC 20555

Flix B. Litton

Materials Engineering Branch

Division of Engineering

Nuclear Regulatory Division

Mail Stop P-1000

Washington, DC 20555

Warren S. Hazelton

Materials Engineering Branch

Division of Engineering

Nuclear Regulatory Division

Mail Stop P-1000

Washington, DC 20555

W. J. Collins

Office of Inspection and Enforcement

Nuclear Regulatory Commission

Washington, DC 20555 
Bob Herman

Office of Inspection and Enf orcement

Nuclear Regulatory Commission Washington, DC 20555

Glen A. Walton

Region I

Office of Inspection and Enf orcement

Nuclear Regulatory Comnission

631 Park Avenue

King of Prussia, PA 19406

Al an R. Herdt

Region II

Office of Inspection and

Enf orcement

Nuclear Regulatory Commission

Suite 3100, 101 Marietta Street NW

Atlanta, GA 30303

Dr. V. Goel

Office of Standards

Nuclear Regulatory Commission

Washington, DC 20555

Lou Frank

Office of Standards

Nuclear Regulatory Commission

Washington, DC 20555

R. W. Weeks

Materials Science Division

Argonne National Laboratory

Argonne, IL 60439

F. Shakir

Department of Metallurgy

Association of American Railroads

3140 S. Federal

Chicago, IL 60616

Mr. L. J. Anderson, B2402

Dow Chemical Company

Texas Division

P. 0. Drawer $K$

Freeport, TX 77541
L. Agree

Electric Power Research Institute 3212 Hillview Avenue

P. 0. Box 10412

Palo Alto, CA 94304

B. R. Sehgal

Electric Power Research Institute 3212 Hillview Avenue

P. 0. Box 10412

Palo Alto, CA 94304

W. L. Pearl

Nuclear Water \& Waste Technology

P. 0. Box 6406

San Jose, CA 95150

M. A. Wolf

Department of Atmospheric Sciences

Oregon State University

Corvallis, OR 97330

D. 0. Harris

Science Applications, Inc.

5 Palo Alto Square, Suite 200

Palo Alto, CA 94304

SM-ALC/MMET

Attn: Capt. John Rodgers

McClellan AFB, CA 95652

Mr. Jerry Whittaker

Union Carbide Company

Oak Ridge National Laboratories

Y 12

Oak Ridge, TN 37830

Dr. Sotirios, J. Vahaviolos

Western Electric, ERC

P. 0. Box 900

Princeton, NJ 08540

Mr. M. C. Jon

Western Electric, ERC

P. 0. Box 900

Princeton, NJ 08540 
P. Caussin

Vincotte

1640 Rhode-Saint-Genese

BELGIUM

ACE Sinclair

Research Division

Berkeley Nuclear Laboratories

Berkeley

Gloucestershire, GL 139 PB

U.K.

Don Birchon

Admiralty Materials Laboratory

Holton Health Poole

Dorser, ENGLAND

020-122-2711

I. P. Bell

Risley Nuclear Labs

UKAEA

Riseley Warrington

Cheshive

U.K.

M. J. Whitt?e

NDT Application Centre

C.E.G.B. Scientific Services

Timpson Road

Manchester M23 9LL

U.K.

0 . For $1 i$

Det Norske Veritas

Veritasveien, 1

PO Box 300

N-1322 Hovik

NORWAY

K. Gott

Studsvik Energiteknic $A B$

S-611 82 Nykoping

SWEDEN
P. Holler

Institut fur Zerstrarangs Frere

Prufverfahren

Univ. Geb. 37

D-6600 Saarbucken

WEST GERMANY

$x$. Edelman

Sulzer Brothers Ltd

Dept. 1513, NDT

$\mathrm{CH}-8401$ Winterthur

\section{ONSITE}

50 Pacific Northwest Laboratory

M. C. Bampton

F. L. Becker (27)

S. H. Bush

R. A. Clark

R. L. Dillon

S. R. Doctor

G. B. Dudder

A. J. Haverfield

P. G. Heasler

P. H. Hutton

C. J. Morris

L. T. Pedersen

S. G. Pitman

G. J. Posakony

G. P. Selby

F. A. Simonen

A. M. Suty

Technical Information (5)

Publishing Coordination SH (2) 



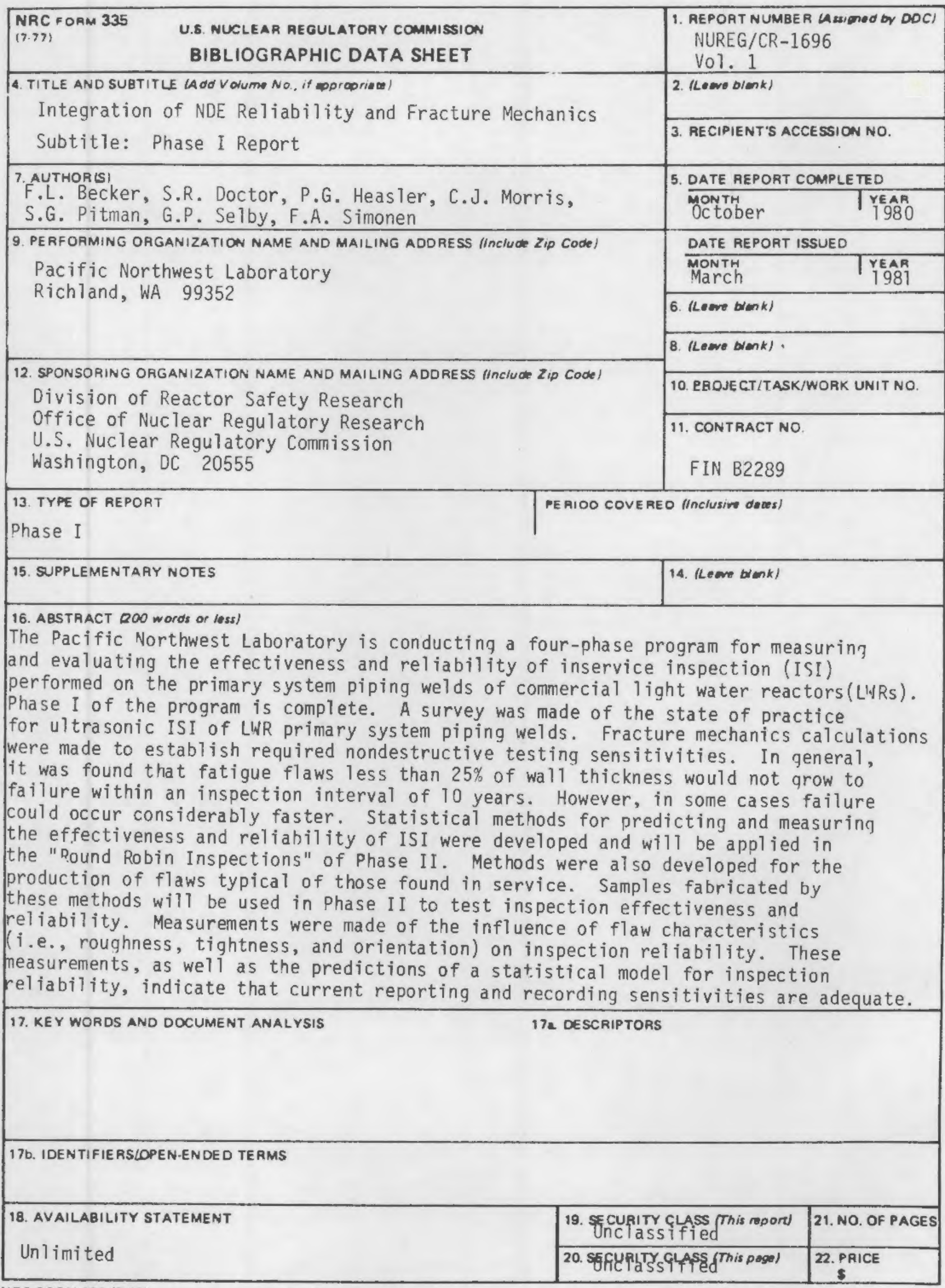


